Fall 2016

\title{
Neutrino Flux Prediction for The Numi Beamline
}

Leonidas Aliaga Soplin

College of William and Mary, laliagasoplin@email.wm.edu

Follow this and additional works at: https://scholarworks.wm.edu/etd

Part of the Physics Commons

\section{Recommended Citation}

Aliaga Soplin, Leonidas, "Neutrino Flux Prediction for The Numi Beamline" (2016). Dissertations, Theses, and Masters Projects. Paper 1477067995.

http://doi.org/10.21220/S2G59J

This Dissertation is brought to you for free and open access by the Theses, Dissertations, \& Master Projects at W\&M ScholarWorks. It has been accepted for inclusion in Dissertations, Theses, and Masters Projects by an authorized administrator of W\&M ScholarWorks. For more information, please contact scholarworks@wm.edu. 
Neutrino Flux Prediction for the NuMl Beamline

\author{
Leonidas Aliaga Soplin \\ Lima, Peru
}

Master of Science, College of William and Mary, 2011

Master of Physics, Pontificia Universidad Catolica del Peru, 2008

Bachelor of Physics, Pontificia Universidad Catolica del Peru, 2002

A Dissertation presented to the Graduate Faculty of the College of William and Mary in Candidacy for the Degree of Doctor of Philosophy

\author{
Department of Physics
}

The College of William and Mary

May 2016 
(C) 2016

Leonidas Aliaga Soplin All rights reserved. 


\section{APPROVAL PAGE}

This Dissertation is submitted in partial fulfillment of the requirements for the degree of

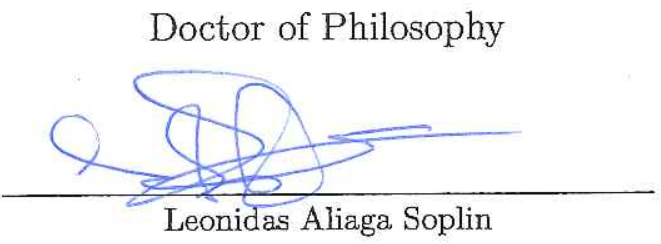

Approved by the Committee, March, 2016

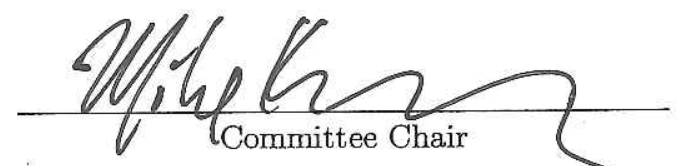

Associate Professor Michael Kordosky, Physies

The College of William and Mary
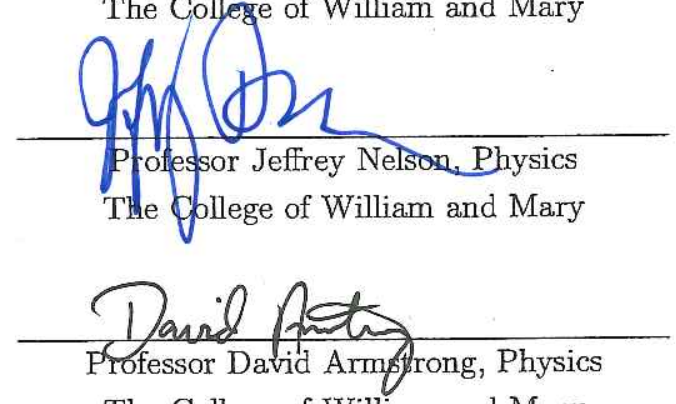

The College of William and Mary
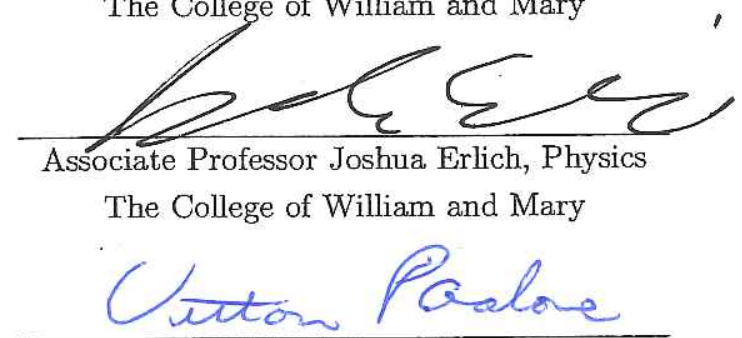

Professor Vittorio Paolone, Physics University of Pittsburgh 


\begin{abstract}
The determination of the neutrino flux in any conventional neutrino beam presents a challenge for the current and future short and long baseline neutrino experiments. The uncertainties associated with the production and attenuation of the hadrons in the beamline materials along with those associated with the beam optics have a big effect in the flux spectrum knowledge. For experiments like MINERvA, understanding the flux is crucial since it enters directly into every neutrino-nucleus cross-sections measurements.

The foundation of this work is predicting the neutrino flux at MINERvA using dedicated measurements of hadron production in hadron-nucleus collisions and incorporating in-situ MINERvA data that can provide additional constraints. This work also includes the prospect for predicting the flux at other detectors like the NOvA Near detector. The procedure and conclusions of this thesis will have a big impact on future hadron production experiments and on determining the flux for the upcoming DUNE experiment.
\end{abstract}




\title{
TABLE OF CONTENTS
}

\begin{abstract}
Acknowledgments . . . . . . . . . . . . . . . . . . .

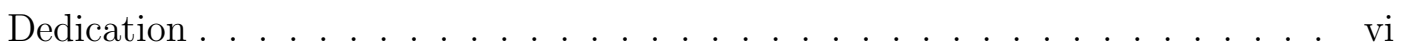

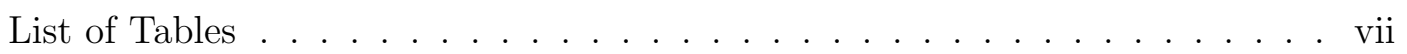

List of Figures . . . . . . . . . . . . . . . ix CHAPTER
\end{abstract}

1 Introduction . . . . . . . . . . . . . . . . 2

1.1 The Standard Model and neutrinos . . . . . . . . . . . . . 3

1.2 Importance of the neutrino flux determination . . . . . . . . 5

2 NuMI beamline . . . . . . . . . . . . . . . . . . . . 15

2.1 Conventional neutrino beams . . . . . . . . . . . . . 15

2.2 NuMI description . . . . . . . . . . . . . . . . . . 20

2.2.1 Primary proton beam . . . . . . . . . . . 21

2.2 .2 Target . . . . . . . . . . . . . . . . . 22

2.2 .3 Magnetic horns . . . . . . . . . . . . . . 25

2.2.4 Decay pipe and Absorbers . . . . . . . . . . . 30

2.2.5 NuMI simulation . . . . . . . . . . . . . . . . 32

2.3 NuMI fluxes . . . . . . . . . . . . . . . . . . . 35

2.3.1 Beam components . . . . . . . . . . . . . 40

2.4 Hadronic cascade in NuMI . . . . . . . . . . . . . . . . . . . . 43

2.4.1 Neutrino ancestries . . . . . . . . . . . . . 43

2.4 Interaction map . . . . . . . . . . . . . . . . . 44

2.4.3 Hadronic production . . . . . . . . . . . . . . . . 48 
2.4 .4 Beam attenuation . . . . . . . . . . . . . . 48

2.5 Focusing uncertainties . . . . . . . . . . . . . . 50

2.5.1 Transverse horn offset . . . . . . . . . . . . . 54

2.5.2 Baffle scraping . . . . . . . . . . . . . 54

2.5.3 POT counting . . . . . . . . . . . . . 55

2.5.4 Horn current . . . . . . . . . . . . . . . . . 55

2.5.5 Horn inner conductor shape . . . . . . . . . . . 55

2.5.6 Longitudinal target offset . . . . . . . . . . . . . 57

2.5.7 Water layer uncertainty . . . . . . . . . . . . 57

3 Overview of neutrino flux determination strategies . . . . . . . . . . 62

3.1 Using external data . . . . . . . . . . . . . . . . . 63

3.2 Using a multi-beam fitting . . . . . . . . . . . . . 65

3.3 Low-nu method . . . . . . . . . . . . . . . . . 67

3.4 Neutrino - electron scattering constraint . . . . . . . . . . 69

3.5 Minerva strategy . . . . . . . . . . . . . . 70

4 Correcting the simulation using hadron production data . . . . . . . . . 72

4.1 Definitions .......................... 73

4.1.1 Total cross-section . . . . . . . . . . . . . . 73

4.1 .2 Differential cross-section . . . . . . . . . . . . . . . 74

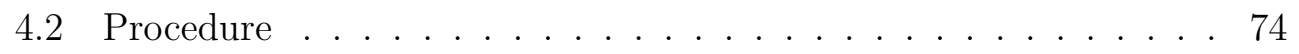

4.2.1 Attenuation Corrections . . . . . . . . . . . . . 75

4.2 .2 Particle Production . . . . . . . . . . . . 77

4.2 .3 Extending data coverage . . . . . . . . . . . . 84

4.2.4 Procedure when there is no data . . . . . . . . . . 87

4.2.5 Uncertainty propagation . . . . . . . . . . . . 90

4.3 Available data . . . . . . . . . . . . . . . . . . . . . 91 
4.4 The thick target datasets . . . . . . . . . . . . . . . . 93

4.4.1 Thick target pion production . . . . . . . . . . . . 95

4.4.2 Thick target kaon production . . . . . . . . . . . . . . . 104

4.5 The thin target hadron production datasets . . . . . . . . . 111

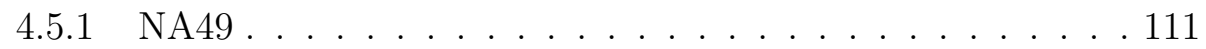

4.5.2 Barton et al. . . . . . . . . . . . . . . . . . . 113

4.5.3 MIPP thin target . . . . . . . . . . . . . . 114

4.5.4 Thin target pion production . . . . . . . . . . . 114

4.5.5 Thin target kaon production . . . . . . . . . . . . . 119

4.5.6 Thin target nucleon production . . . . . . . . . . . 123

4.6 Thin target data extension in Generation 2 . . . . . . . . . . . 131

4.6.1 Interactions of nucleons not covered by thin target data . . 134

4.6.2 Incident mesons and other interactions . . . . . . . . . . . 134

4.7 Thin target inelastic and absorption cross-section datasets . . . . 136

4.7.1 Proton - carbon inelastic cross-section . . . . . . . . . 136

4.7.2 $\pi^{ \pm}$absorption cross-sections . . . . . . . . . . . 136

4.7.3 $K^{ \pm}$absorption cross-sections . . . . . . . . . . . . 137

4.7.4 Technical implementation of the absorption and inelastic cross-section . . . . . . . . . . . . . . . 138

$4.8 \quad \mathrm{PPFX} \ldots \ldots \ldots \ldots \ldots \ldots$

4.8 .1 MakeReweight . . . . . . . . . . . . . . 145

4.8.2 Data and MC Inputs . . . . . . . . . . . . . . . . . . 147

4.8.3 InteractionChainData . . . . . . . . . . . . . . 148

4.8 .4 ReweightDriver . . . . . . . . . . . . . . . 148

4.8.5 Reweighters . . . . . . . . . . . . . . . . . 148 
5 A priori MINERvA Low Energy Beam results . . . . . . . . . . . . . 151

5.1 MINERvA flux Generation 0 . . . . . . . . . . . . . 152

5.2 MINERvA flux Generation 1 . . . . . . . . . . . . . 155

5.3 MINERvA flux Generation 2 . . . . . . . . . . . . 157

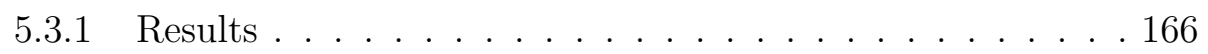

5.4 Discussion . . . . . . . . . . . . . . . . . . 179

6 Additional flux constraining using MINERvA in-situ measurements . . 183

6.1 Low-nu flux at MINERvA . . . . . . . . . . . . . . 184

6.1.1 Generation 2 vs low-nu flux comparison . . . . . . . . . 188

6.2 Additional constraint using neutrino electron scattering events . . 192

6.3 Comparison of Gen2-thick with Generation 0 and Generation 1 . 197

7 Medium Energy Beam results . . . . . . . . . . . . . . 205

7.1 Gen2-thin flux for ME MINERvA . . . . . . . . . . . 206

7.2 Gen2-thin for NOvA ND . . . . . . . . . . . . . . . . 212

8 Conclusions . . . . . . . . . . . . . . . . . . 217

APPENDIX A

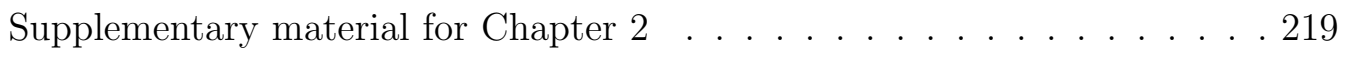

A.1 Focusing components . . . . . . . . . . . . . . . . 219

A.2 Flux HP leading to neutrino in NOvA . . . . . . . . . . . 227 APPENDIX B

NuMI flux tables . . . . . . . . . . . . . . . . . . . . . 235

Bibliography ... . . . . . . . . . . . . . . . . 240

Vita . . . . . . . . . . . . . . . . . . . . . . . . . . . . . . . . . . . . . . 


\section{ACKNOWLEDGMENTS}

I would like to thank my parents, Leonidas and Isabel, for their love and for always take care of my sister Jessica and me. To my sister and her amazing children, Belen, Isabella, Nicolas and Fabiano, for their support.

I would like to express my special appreciation and gratitude to my advisor, Mike Kordosky, for his guidance and patience throughout my research. My gratitude to all members of the MINERvA Collaboration for their suggestions during my research.

And thanks to all my friends, those in my country, Peru, and those that I met in US. Special thanks to Anne Norrick, for her friendship and all her help. 
I present this thesis in honor of my parents. 


\section{LIST OF TABLES}

1.1 Oscillation parameter status . . . . . . . . . . . . . . . 7

2.1 Main decay modes into neutrinos . . . . . . . . . . . . . 16

2.2 LE and ME target parameters . . . . . . . . . . . . . . . 24

2.3 LE NuMI runs . . . . . . . . . . . . . . . . . . . . . . . . . . . 36

2.4 Relevant volumes for hadron production correction . . . . . . . . . 50

2.5 MINERvA LE flux playlists . . . . . . . . . . . . . . . 58

4.1 Material scaling uncertainties . . . . . . . . . . . . 87

4.2 NA49 systematic uncertainties . . . . . . . . . . . . . . . 113

4.3 Meson absorption cross-section uncertainties implemented in Generation . . . . . . . . . . . . . . . . . . . 144

4.4 PPFX reweighters . . . . . . . . . . . . . . . 150

5.1 Flux used in MINERvA publications . . . . . . . . . . . . 157

5.2 Integrated flux for $\nu_{\mu}$ and $\bar{\nu}_{\mu}$ in Gen2-thin and Gen2-thick . . . . . 179

5.3 Disagreement between Gen2-thin and Gen2-thick for $\nu_{\mu} \ldots \ldots$. . 182

6.1 Low-nu bins . . . . . . . . . . . . . . . . . . . . . . . . 186

$6.2 \chi 2 / N D F$ for Generation 2 and low-nu difference . . . . . . . . . . . 191

$6.3 \chi 2 / N D F$ for Gen2-thick and Gen2-thin difference . . . . . . . . . 192

6.4 Integrated flux ratios between generations . . . . . . . . . . . 198 
7.1 Integrated fluxes for Gen2-thin . . . . . . . . . . . . . 207

B.1 Generation 2 - thin LE flux results at MINERvA . . . . . . . . . 236

B.2 Generation 2 - thick LE flux results at MINERvA . . . . . . . . . . . 237

B.3 Generation 2 - thin ME flux results at MINERvA . . . . . . . . . 238

B.4 Generation 2 - thin ME flux results at NOvA ND . . . . . . . . . . 239 


\section{LIST OF FIGURES}

$1.1 \mathrm{CC}$ and NC Feynman diagrams . . . . . . . . . . . . . . 5

1.2 A two-detector neutrino oscillation experiment . . . . . . . . . 8

$1.3 \mathrm{~F} / \mathrm{N}$ MINOS flux $\ldots \ldots \ldots \ldots . \ldots \ldots$

1.4 Charged inclusive neutrino cross-section per nucleon . . . . . . . . . . 12

1.5 Coherent $\pi^{ \pm}$production cross-section . . . . . . . . . . . . . . . . . 14

2.1 Energy relation between a neutrino and its meson parent . . . . . . . 17

2.2 Diagram of a horn and a magnetic horn . . . . . . . . . . . . . . 18

2.3 NuMI beamline schematic view . . . . . . . . . . . . . . 20

2.4 Plan elevation view of NuMI Beam Facility . . . . . . . . . . . . 22

2.5 Photograph of the LE NuMI target . . . . . . . . . . . . . . . 22

2.6 Sketch of the LE and ME targets . . . . . . . . . . . . . 23

2.7 Photograph of the Horn $1 \ldots \ldots . \ldots . \ldots . \ldots 25$

2.8 Horn 1 magnetic field . . . . . . . . . . . . . . . . . . 27

2.9 Focusing components . . . . . . . . . . . . . . . . . . . . 29

2.10 Focusing components for LE010z185i . . . . . . . . . . . . . . . 29

$2.11 \pi^{+}$and $K^{-} \nu_{\mu}$ parent focusing component ratios for LE010z185i . . . 31

$2.12 \mathrm{MC} \nu_{\mu}$ fluxes for LE runs . . . . . . . . . . . . . . . . . 37

$2.13 \pi^{+}$production in the target . . . . . . . . . . . . 39

$2.14 \mathrm{MC} \nu_{\mu}$ fluxes for ME runs . . . . . . . . . . . . . . . . 40

2.15 Neutrino components for LE and ME beams . . . . . . . . . . . . . . 42 
2.16 Neutrino ancestries at MINERvA . . . . . . . . . . . . . 45

2.17 Interaction map for $\nu_{\mu}$ in MINERvA . . . . . . . . . . . . . 46

2.18 Incident protons for $\nu_{\mu}$ in MINERvA . . . . . . . . . . . . . 47

2.19 Hadron production in $\mathrm{pC}$ leading to $\nu_{\mu}$ flux in MINERvA . . . . . . . 49

2.20 Material traversed by particles leading to $\nu_{\mu}$ flux in MINERvA . . . . 51

2.21 LE010z185i focusing fractional shifts . . . . . . . . . . . 53

2.22 Effect of the new horn model _ . . . . . . . . . . . . . 56

2.23 Longitudinal target position effect . . . . . . . . . . . . . . . . 59

2.24 Water layer draws . . . . . . . . . . . . . . . . . . . 60

2.25 Water layer effect . . . . . . . . . . . . . . . . . 61

3.1 Hadron production experiments for neutrino flux (HARP and NA61) 66

3.2 Minos beam fit results . . . . . . . . . . . . . . . . . 67

3.3 neutrino - electron scattering Feynman diagram . . . . . . . . . . 69

4.1 Particle beam traversing a volume . . . . . . . . . . . 76

4.2 The survival correction . . . . . . . . . . . . . . 76

4.3 Attenuation correction when an interaction happened $(\sigma=193.6 \mathrm{mb}) \quad 78$

4.4 Attenuation correction when an interaction happened $\left(\sigma_{M C}=368 \mathrm{mb}\right) 78$

4.5 FLUKA energy scaling correction . . . . . . . . . . . . . . . 79

4.6 Scaling NA49 to NA61 . . . . . . . . . . . . . . . . . . . 81

4.7 Fractional difference between NA49 and NA61 applying scaling . . . . 82

4.8 Skubic data fit . . . . . . . . . . . . . . . . . 86

4.9 Example of geant4 model spread . . . . . . . . . . . . . . . . . 88

4.10 Example of the multi-universe technique . . . . . . . . . . . . 91 
4.11 Schematic view of MIPP detector . . . . . . . . . . . . . . 94

4.12 Interactions per $\pi^{+}$that exits the target for $p_{Z}$ in $0-20 \mathrm{GeV} / \mathrm{c}$. . . . 96

4.13 Interactions per $\pi^{+}$and $K^{+}$that exit the target for $p_{Z}$ in $20-80 \mathrm{GeV} / \mathrm{c} 97$

4.14 MIPP NuMI $\pi^{+}$and $\pi^{-}$data . . . . . . . . . . . . . . . . . . 98

4.15 MIPP NuMI $\pi$ data statistical uncertainties . . . . . . . . . . . . . 99

4.16 MIPP NuMI $\pi$ data systematic uncertainties . . . . . . . . . . . . . 100

4.17 Bin-to-bin correlation effect on MIPP NuMI systematic uncertainties 101

4.18 MIPP NuMI charged pions weights . . . . . . . . . . . . . . 103

4.19 MIPP NuMI yield ratios . . . . . . . . . . . . . . . . . . 104

4.20 MIPP NuMI $K / \pi$ statistical uncertainties $\ldots . . . . . . . .105$

4.21 MIPP NuMI $K / \pi$ systematic uncertainties $\ldots \ldots$. . . . . . . . . . . 107

4.22 MIPP NuMI charged kaons weights . . . . . . . . . . . . . . . 109

4.23 MIPP NuMI neutral kaons weights . . . . . . . . . . . . . . . 110

4.24 Schematic view of NA49 detector . . . . . . . . . . . . . . 112

4.25 NA49 charged pion production in proton - carbon interactions . . . . 115

4.26 Barton et al $\pi^{+}$data in proton - carbon interactions . . . . . . . . 116

4.27 NA49 statistical uncertainty for pion production . . . . . . . . . 118

4.28 Thin target weights for charged pions . . . . . . . . . . . . . . 120

4.29 NA49 charged kaon production in proton carbon interactions . . . . . 121

4.30 MIPP pC yield ratios . . . . . . . . . . . . . . . . . . . 122

4.31 NA49 statistical uncertainty for kaon production . . . . . . . . . . . 124

4.32 MIPP pC statistical uncertainties for $K / \pi \ldots \ldots$. . . . . . 125

4.33 MIPP pC systematic uncertainties for $K / \pi \ldots \ldots$. . . . . . 126

4.34 Thin target weights for charged kaons . . . . . . . . . . . . . . 127 
4.35 Thin target weights for neutral kaons . . . . . . . . . . . . . 128

4.36 NA49 proton production in proton carbon interactions . . . . . . 130

4.37 NA49 neutron production in proton carbon interactions . . . . . . . 131

4.38 NA49 nucleon statistical uncertainties . . . . . . . . . . . . . . . . 132

4.39 Thin target weights for nucleon production . . . . . . . . . . . 133

4.40 Proton-carbon inelastic cross-sections. . . . . . . . . . . . . . 137

$4.41 \pi \mathrm{C}$ and $\pi \mathrm{Al}$ data and $\mathrm{MC}$ absorption cross-section . . . . . . . . . . 138

$4.42 \pi$ data - MC absorption cross-section data - MC comparisons . . . . . 139

4.43 Charged kaon on $\mathrm{C}$ and $\mathrm{Al}$ data and MC absorption cross-section . . 140

$4.44 K^{+}$data - MC absorption cross-section data - MC comparisons . . . 141

$4.45 K^{-}$data - MC absorption cross-section data - MC comparisons . . . 142

4.46 PPFX flowchart. . . . . . . . . . . . . . . . . . 146

5.1 Generation 0 flux . . . . . . . . . . . . . . . . . . 154

5.2 Generation 1 flux . . . . . . . . . . . . . . . . . 156

5.3 Generation 0 and Generation 1 flux fractional uncertainties . . . . . . 158

5.4 Interactions per neutrino in Gen2-thin . . . . . . . . . . . . . . . 162

5.5 Interactions per neutrino in Gen2-thick . . . . . . . . . . . . . . 163

5.6 Nucleon-A interaction per neutrino covered in Generation 2 . . . . . . 165

5.7 NuMI material traversed . . . . . . . . . . . . . . 167

5.8 Gen2-thin $\nu_{\mu}$ flux $\ldots \ldots \ldots \ldots$

5.9 Gen2-thick $\nu_{\mu}$ flux . . . . . . . . . . . . . . . . 170

5.10 Gen2-thin $\bar{\nu}_{\mu}$ flux . . . . . . . . . . . . . . . . . 171

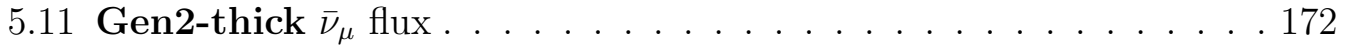

5.12 Gen2-thin $\nu_{e}$ flux $\ldots \ldots \ldots$. . . . . . . . . . . . . . . . . . . . .

xii 
5.13 Gen2-thick $\nu_{e}$ flux . . . . . . . . . . . . . . . . . . 174

5.14 Fractional uncertainties for Gen2-thin . . . . . . . . . . . . 176

5.15 Fractional uncertainties for Gen2-thick . . . . . . . . . . . . 177

5.16 Energy bin to bin flux correlation . . . . . . . . . . . . . 178

5.17 Flux integral correlation for Gen2-thin . . . . . . . . . . . 180

5.18 Flux integral correlation for Gen2-thick . . . . . . . . . . . . 180

$5.19 \nu_{\mu}$ Gen2-thin and Gen2-thick comparison . . . . . . . . . . 181

$5.20 \bar{\nu}_{\mu}$ Gen2-thin and Gen2-thick comparison . . . . . . . . . . . 181

6.1 Overlap and $\nu$ cross-sections . . . . . . . . . . . . . . . . . 185

$6.2 \nu_{\mu}$ low-nu flux . . . . . . . . . . . . . . . . . 187

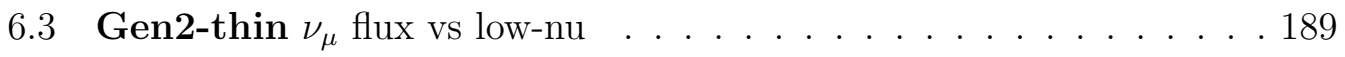

6.4 Gen2-thick $\nu_{\mu}$ flux vs low-nu . . . . . . . . . . . . . . . . 190

6.5 Electron energy distribution from $\nu$-e scattering . . . . . . . . . 193

6.6 Effect of the $\nu e$ constraint on the Generation 1 integrated flux . . . . 195

6.7 Effect of the $\nu e$ constraint on the Gen2-thin flux . . . . . . . . . . . 196

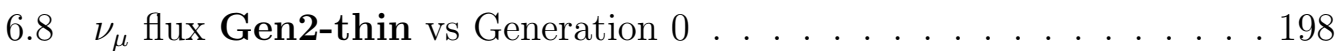

$6.9 \bar{\nu}_{\mu}$ flux Gen2-thin vs Generation $0 \ldots \ldots 9$

$6.10 \nu_{\mu}$ flux Gen2-thin vs Generation $1 \ldots 200$

$6.11 \bar{\nu}_{\mu}$ flux Gen2-thin vs Generation $1 \ldots . . \ldots 201$

$6.12 \nu_{e}$ flux Gen2-thin vs Generation $1 \ldots 202$

$6.13 \nu_{\mu}$ flux Gen2-thin vs Generation $1+$ neutrino electron scattering constraint . . . . . . . . . . . . . . . . . 203

$6.14 \nu_{e}$ flux Gen2-thin vs Generation $1+$ neutrino electron scattering constraint . . . . . . . . . . . . . . . . . . 204 
7.1 ME PPFX coverage for $\nu_{\mu}$ in MINERvA . . . . . . . . . . . 208

7.2 ME Gen2 - thin $\nu_{\mu}$ flux at MINERvA . . . . . . . . . . . . . . . 209

7.3 Fractional Uncertainties for $\nu_{\mu}$ ME Gen2-thin flux in MINERvA. . . 210

7.4 Bin to bin correlation of ME Gen2-thin at MINERvA. . . . . . . . . 211

7.5 ME Gen2-thin interactions and material coverages for $\nu_{\mu}$ in NOvA . 213

7.6 Gen2-thin ME NOvA flux . . . . . . . . . . . . . . . 214

7.7 Fractional Uncertainties for $\nu_{\mu}$ ME Gen2 - thin flux in NOvA. . . . . 215

7.8 Comparison between Gen2-thin and the FLUGG $\nu_{\mu}$ flux NOvA ND 216

A.1 Longitudinal vs. transverse momentum yield per $\pi^{+} \nu_{\mu}$ parent . . . . 220

A.2 Unfocused components of $\pi^{+} \nu_{\mu}$ parent . . . . . . . . . . . 221

A.3 H2 only components of $\pi^{+} \nu_{\mu}$ parent . . . . . . . . . . . . . . . 222

A.4 Horn1 only components of $\pi^{+} \nu_{\mu}$ parent . . . . . . . . . . . . 223

A.5 Underfocused components of $\pi^{+} \nu_{\mu}$ parent . . . . . . . . . . . . 224

A.6 Overfocused components of $\pi^{+} \nu_{\mu}$ parent . . . . . . . . . . . 225

A.7 Other components of $\pi^{+} \nu_{\mu}$ parent . . . . . . . . . . . . . 226

A.8 Focusing components for NOvA ND . . . . . . . . . . . . . . . . 228

A.9 Muon and electron neutrino parents in NOvA . . . . . . . . . . . . 229

A.10 Muon and electron neutrino grandparents in NOvA . . . . . . . . . . 230

A.11 Interaction map for $\nu_{\mu}$ in NOvA . . . . . . . . . . . . . . 231

A.12 Incident protons for $\nu_{\mu}$ in NOvA . . . . . . . . . . . . . . 232

A.13 Hadron production leading to $\nu_{\mu}$ in NOvA . . . . . . . . . . . 233

A.14 Beam attenuation for particles leading to $\nu_{\mu}$ in NOvA . . . . . . . 234 
NEUTRINO FLUX PREDICTION FOR THE NUMI BEAMLINE 


\section{CHAPTER 1}

\section{Introduction}

The determination of the neutrino flux in any conventional neutrino beam presents a challenge for the current and future short and long baseline neutrino experiments. The uncertainties associated with the production and attenuation of the hadrons in the beamline materials along with those associated with the beam optics have a big effect on the knowledge of the flux spectrum. For neutrino-scattering experiments like MINERvA, understanding the flux is crucial since it enters directly into every neutrino-nucleus cross-section measurement.

This thesis is about the prediction of the neutrino flux for NuMI beamline. The foundation of this work is the use of dedicated measurements of hadron production in hadron-nucleus collisions in the beamline to constraint the flux for MINERvA, and incorporating in-situ MINERvA data as additional constraints. This work also includes the prospect for predicting the flux at other detectors like the NOvA.

After a brief introduction of the neutrinos in the Standard Model, this chapter is dedicated to present the importance of the flux determination for neutrino oscillation and neutrino cross-section experiments. 


\subsection{The Standard Model and neutrinos}

The Standard Model of particle physics describes, in a simple structure, the matter in the universe in terms of its fundamental constituents and their interactions (for more details Ref. [1]). The matter consists of fermions that can be classified as leptons and quarks depending on their intrinsic properties and how they interact with each other by exchanging gauge bosons. The gauge bosons are gamma $(\gamma)$ for electromagnetic interactions, gluon $(g)$ for strong interactions and, $Z^{0}$ and $W^{ \pm}$for weak interactions. Every particle also has an antiparticle with all internal quantum numbers inverted. The model also includes a boson called Higgs $(H)$ that plays a key role to explain the existence of the particle's masses. There are six types of quarks: up $(u)$, down $(d)$, charm $(c)$, strange $(s)$, top $(t)$ and bottom $(b)$ and they have strong, weak and electromagnetic interactions. They are grouped into three generations of doublets. The upper quarks have charge equal to $\frac{2}{3}$ and the lower quarks have charge equal to $-\frac{1}{3}$.

$$
\left(\begin{array}{l}
u \\
d
\end{array}\right) \quad\left(\begin{array}{l}
c \\
s
\end{array}\right) \quad\left(\begin{array}{l}
t \\
b
\end{array}\right)
$$

Quarks are always confined in hadrons and bond by the strong interaction. Hadrons can be classified into mesons and baryons. Mesons are unstable particles composed by one quark and one antiquark such as pion plus $(u \bar{d})$ and kaon plus $(u \bar{s})$, and baryons are composed by three quarks. The most abundant baryons are protons and neutrons, that are the basic part of the atomic nucleus of ordinary matter. Protons and neutrons (nucleons) are comprised by the lightest quarks ( $u$ and $d$ ) and they are $u u d$ and $u d d$, respectively.

There are six types of leptons, three with charge equal to -1 : electron $(e)$, muon $(\mu)$ and tau $(\tau)$, and three neutral, each one associated with one charged lepton: 
electron neutrino $\left(\nu_{e}\right)$, muon neutrino $\left(\nu_{\mu}\right)$ and tau neutrino $\left(\nu_{\tau}\right)$. Charged leptons can interact by electromagnetic and weak interactions. Neutrinos only interact (and be produced) weakly. Leptons can be grouped into three generations of doublets:

$$
\left(\begin{array}{c}
e \\
\nu_{e}
\end{array}\right) \quad\left(\begin{array}{c}
\mu \\
\nu_{\mu}
\end{array}\right) \quad\left(\begin{array}{c}
\tau \\
\nu_{\tau}
\end{array}\right)
$$

There are two possible channels depending on which boson is exchanged when a neutrino interacts with the matter. When the boson exchanged is a $W$, the channel is called charged-current (CC). In this channel, the neutrino is converted to the charged lepton of its same generation and the matter, for instance a quark inside of a nucleon, is also converted. When the boson exchanged is a $Z$, the channel is called neutral-current (NC) and no conversion occur.

Figure 1.1 shows two Feynman diagrams, the left one for charged-current and the right one for neutral-current channels. The index $\alpha$ can be $e^{-}, \mu^{-}$or $\tau^{-}$. For CC, the exchange of a $W^{-}$with a neutron produces a neutrino conversion, for instance, $\nu_{\mu} \rightarrow \mu^{-}$and $d$ quark into an $u$ quark, making a proton in the final state. For NC, a $Z^{0}$ boson is exchanged between a neutrino and a fermion $(f)$.

Neutrinos are considered massless in the Standard Model. However, since the late 1990's, there has been overwhelming evidence that the three types of neutrinos, oscillate when they travel through vacuum or matter. The neutrino oscillation is a quantum mechanical phenomenon that implies that the neutrino are massive and mixed. Then the Standard Model has to be extended to include the neutrino mass $[2]$.

Many experiments have been developed to determine the parameters that describe the neutrino oscillation. In addition to the study of the neutrinos from the Sun and those generated in the Earth's atmosphere, neutrinos have been generated in reactors and using particle accelerators [3]. Precisely, the flux determination of 


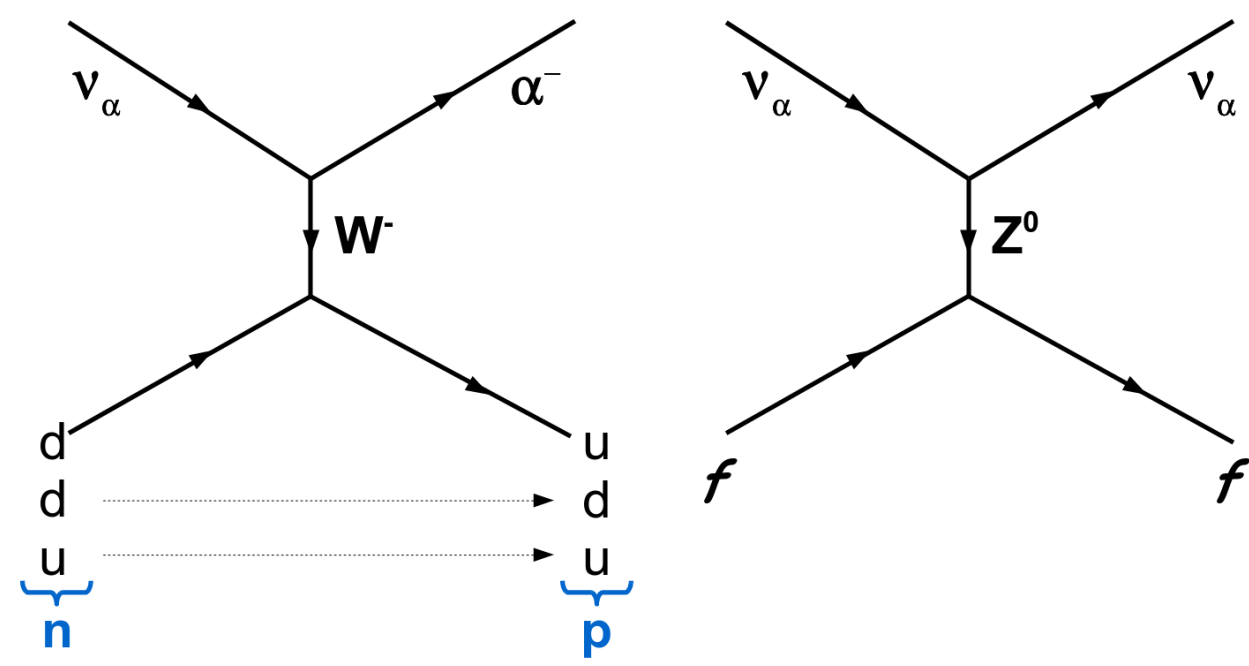

FIG. 1.1: Feynman diagrams of charged-current (left) and neutral-current (right) neutrino interaction channels. $\alpha$ represents $e, \mu, \tau$, and $f$ is any fermion.

neutrinos generated using particle accelerators is the topic of this thesis. The importance of this work for the neutrino oscillation studies and neutrino cross-section determination will be presented in the next section.

\subsection{Importance of the neutrino flux determina- tion}

The weak eigenstates of a neutrino, $\nu_{\alpha}$, are linear combinations of the mass eigenstates $\nu_{i}$

$$
\left|\nu_{\alpha}\right\rangle=\sum_{i=1}^{3} U_{\alpha i}^{*}\left|\nu_{i}\right\rangle
$$

where $U$ is the mixing unitary matrix known as the PMNS matrix (PontecorvoMaki-Nakagawa-Sakata). The probability of a neutrino be detected as $\nu_{\beta}$ after a time $t$ (or distance, for relativistic neutrinos) is given by: 


$$
P_{\nu_{\alpha} \rightarrow \nu_{\beta}}=\left|\left\langle\nu_{\beta} \mid \nu_{\alpha}(t)\right\rangle\right|^{2}=\left|\sum_{i=1}^{3} \sum_{j=1}^{3} U_{\alpha i}^{*} U_{\beta j}\left\langle\nu_{j}(0) \mid \nu_{i}(t)\right\rangle\right|^{2}
$$

In many circumstances, considering only the oscillation between two neutrinos is a good approximation such as in the $\nu_{\mu}$ oscillation study at long baselines in conventional neutrino beams. In these cases, the mixing matrix can be parametrized in terms of the angle $\theta$ :

$$
\left(\begin{array}{cc}
\cos \theta & \sin \theta \\
-\sin \theta & \cos \theta
\end{array}\right)
$$

The two-neutrino oscillation probability is given by:

$$
P_{\nu_{\alpha} \rightarrow \nu_{\beta}}=\sin ^{2}(2 \theta) \sin ^{2}\left(\frac{1.27 \Delta m_{i j}^{2} L}{E}\right)
$$

where $L$ is the distance traveled by the neutrino and $E$, its energy. The factor "1.27" supposes that $E$ is expressed in $\mathrm{GeV}, L$ in $\mathrm{km}$ and the squared neutrino mass difference $\Delta m^{2}$ in $\mathrm{eV}^{2} / \mathrm{c}^{4}$ units.

In the three-neutrino oscillation scenario, the PMNS matrix can be expressed as:

$$
U=\left(\begin{array}{ccc}
1 & 0 & 0 \\
0 & \cos \theta_{23} & \sin \theta_{23} \\
0 & -\sin \theta_{23} & \cos \theta_{23}
\end{array}\right)\left(\begin{array}{ccc}
\cos \theta_{13} & 0 & \sin \theta_{13} e^{\delta_{C P}} \\
0 & 1 & 0 \\
-\sin \theta_{13} e^{\delta_{C P}} & 0 & \cos \theta_{13}
\end{array}\right)\left(\begin{array}{ccc}
\cos \theta_{12} & \sin \theta_{12} & 0 \\
-\sin \theta_{12} & \cos \theta_{12} & 0 \\
0 & 0 & 1
\end{array}\right)
$$

Considering the Equations 1.3 and 1.6, there are 7 independent parameters in the three-neutrino oscillation scenario: three masses $\left(m_{1}, m_{2}\right.$ and $\left.m_{3}\right)$, three missing angles $\left(\theta_{12}, \theta_{23}\right.$ and $\left.\theta_{13}\right)$ and one charge-parity $(\mathrm{CP})$ violation phase $(\delta)$. Neutrino oscillation experiments can only measure $\Delta m_{i j}^{2}$ (See Equation 1.5 and in Ref. [2] for three-neutrino oscillation probability).

The current status of these parameters are listed in table 1.1. $\Delta m^{2}=m_{3}^{2}-$ 
$\left(m_{2}^{2}+m_{1}^{2}\right) / 2$. When $m_{1}<m_{2}<m_{3}$ (it is called normal hierarchy), $\Delta m^{2}>0$. When $m_{3}<m_{1}<m_{2}$ (the values are shown in brackets), $\Delta m^{2}>0$ (it is called inverse hierarchy).

\begin{tabular}{|c|c|}
\hline Parameter & best-fit $( \pm \sigma)$ \\
\hline$\Delta m_{21}^{2}\left[10^{-5} \mathrm{eV}^{2}\right]$ & $7.54_{-0.22}^{+0.26}$ \\
$\Delta m^{2}\left[10^{-3} \mathrm{eV}^{2}\right]$ & $2.43 \pm 0.06(2.38 \pm 0.06)$ \\
$\sin ^{2} \theta_{12}$ & $0.308 \pm 0.017$ \\
$\sin ^{2} \theta_{23}, \Delta m^{2}>0$ & $0.437_{-0.023}^{+0.033}$ \\
$\sin ^{2} \theta_{23}, \Delta m^{2}<0$ & $0.455_{-0.031}^{+0.039}$ \\
$\sin ^{2} \theta_{13}, \Delta m^{2}>0$ & $0.0234_{-0.0019}^{+0.0020}$ \\
$\sin ^{2} \theta_{13}, \Delta m^{2}<0$ & $0.0240_{-0.0022}^{+0.0019}$ \\
$\delta / \pi(2 \sigma$ range $)$ & $1.39_{-0.27}^{+0.38}\left(1.31_{-0.33}^{+0.29}\right)$ \\
\hline
\end{tabular}

TABLE 1.1: Oscillation parameter status taken from Ref. [3] based on the global fit made by Ref. [4].

Long-baseline neutrino experiments use a conventional neutrino beam (described in Chapter 2) and a pair of detectors, one close to the neutrino production point (the near detector) and the other further away (the far detector). The location of the far detector and the beam energy spectrum are chosen to measure the oscillation maxima of Equation 1.4. Having the two detectors share the same beam reduces systematic uncertainties due to the neutrino flux, the cross-section model, and potentially also the detector performance. Figure 1.2 shows a sketch of this two-detector strategy.

If the cross-section is well-understood, the oscillation probability can be calculated as:

$$
P\left(E_{i}\right)=\left(\frac{N_{2}}{N_{1}}\right)_{i}\left(\frac{A_{1}}{A_{2}}\right)\left(\frac{\phi_{1}}{\phi_{2}}\right)_{i}
$$

where $A_{1}$ and $A_{2}$ represent the acceptance and efficiency of each detector, $\phi_{1}$ and $\phi_{2}$ are the neutrino fluxes at each detector, and $\mathrm{N}_{1}$ and $\mathrm{N}_{2}$ are the events 


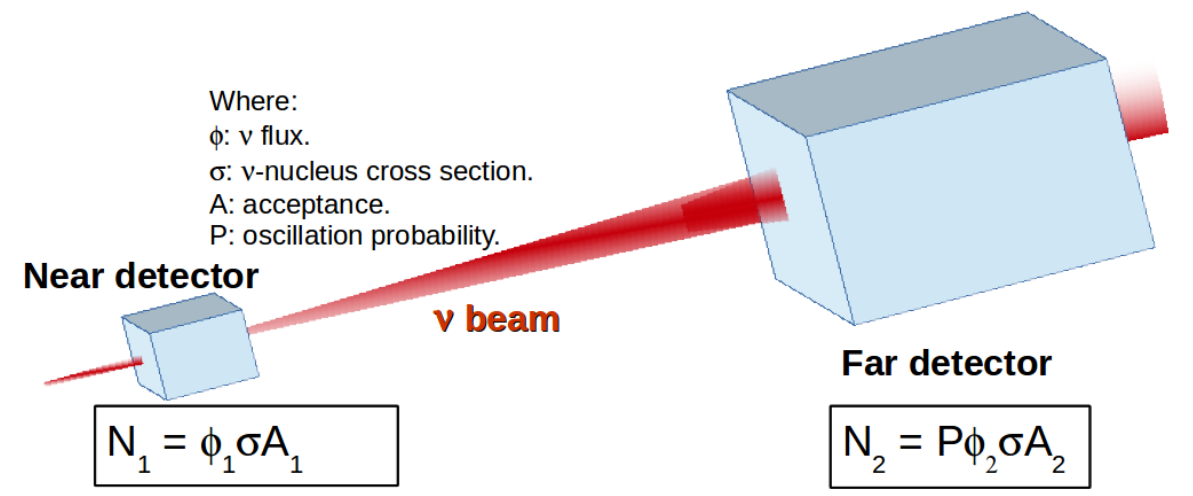

FIG. 1.2: A two-detector neutrino oscillation experiment.

measured in a particular energy bin.

However, this is not so simple. The flux uncertainty only partially cancels since the near detector sees a distributed neutrino source and the far detector sees a point neutrino source (for instance, the MINOS far detector is $735 \mathrm{~km}$ away). The crosssections do not necessarily cancel if the detectors are not made of the same materials with the same response and acceptance.

As an example, Figure 1.3 shows the ratio of the flux at the MINOS far detector divided by the flux at the MINOS near detector (the inverse of $\frac{\phi_{1}}{\phi_{2}}$ in Equation 1.7). The white boxes in the figure represent the prediction from the MINOS flux simulation. However, after MINOS tuned their simulation by constraining the hadron production in the target (see later Section 3.2), the shape of the ratio changes. That means that knowing the flux and how well the underlying physics model is representing nature is absolutely relevant for the accuracy of the oscillation parameters.

On the other hand, the cross-sections are also a source of uncertainty for neutrino oscillation experiments. In particular, the 0.1-20 $\mathrm{GeV}$ region where many experiments are running is complicated because the neutrino-nucleus cross-sections are not well known, as can be seen in Figures $1.4 \mathrm{a}$ and $1.4 \mathrm{~b}$. This region is also 


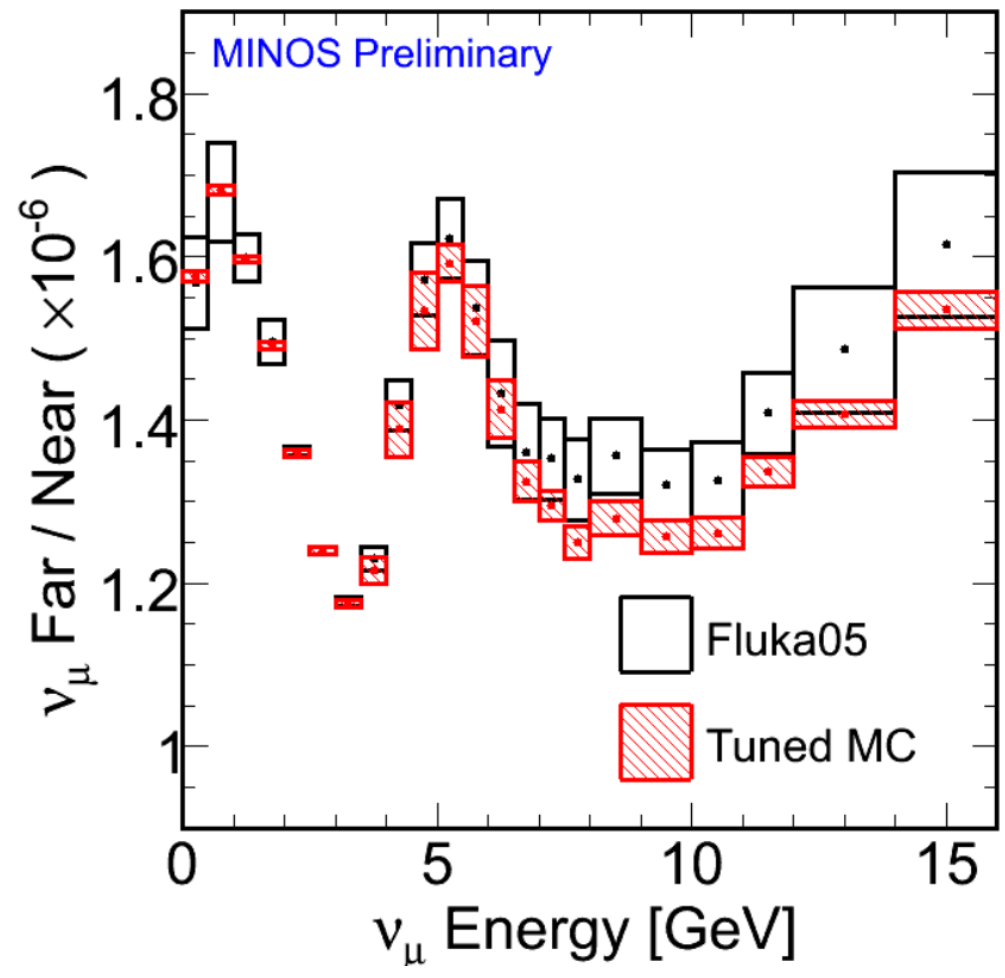

FIG. 1.3: MINOS far over near flux ratio vs. neutrino energy. Plot taken taken from [5]. 
relevant because is the transition between neutrino scattering mechanisms $[6,7]$ :

- Quasi-elastic scattering. In this process the neutrino scatters off an entire nucleon rather than a quark. This process is dominant for energy less than $2 \mathrm{GeV}$. The left diagram of Figure 1.1 shows an example for the CC channel. For muon neutrinos (similar for muon antineutrinos ${ }^{1}$ ),

$$
\nu_{\mu} n \rightarrow \mu^{-} p
$$

In the NC channel, the neutrinos scatters elastically from the nucleons,

$$
\nu p \rightarrow \nu p, \quad \nu n \rightarrow \nu n
$$

- Resonance production. In this process, the neutrino excite a struck nucleon to an excited state and then it decays quickly to a nucleon and a single pion. This channel becomes predominant when the neutrino have enough energy to produce a Delta baryon $(\Delta)^{2}$,

$$
\nu_{\mu} N \rightarrow \mu^{-} \Delta \quad \text { and } \quad \Delta \rightarrow \pi N^{\prime}
$$

where $N, N^{\prime}=n, p$. Higher multiplicities are also possible.

Neutrinos can also produce single pion coherently, by scattering from the entire nucleus and transferring a small amount of energy to the nucleus,

$$
\nu_{\mu} A \rightarrow \nu_{\mu} A \pi^{0} \quad \nu_{\mu} A \rightarrow \mu^{-} A \pi^{+}
$$

- Deep inelastic scattering (DIS). When the neutrinos have large momentum

\footnotetext{
${ }^{1}$ For antineutrino, Hyperion production is also possible [6]

${ }^{2}$ The mass of the $\Delta$ is $1.232 \mathrm{GeV}$ and its lifetime is on the order of $10^{-24}[s]$
} 
transfer they can resolve the internal structure of the nucleon and interact with a quasi-free quark within the nucleon. After a quark is stuck a hadronization process begins and lead a formation of a complex final state hadrons. For high neutrinos energies (between 5-10 GeV), the neutrino interactions are dominated by DIS.

As can be seen in Figure 1.4, the datasets have large uncertainties and even disagreements in some energies. The source of this problem could come from a bad understanding of the nuclear effects. Future experiments like DUNE will consist of liquid Argon (LAr) and it is crucial to understand the nuclear effects in the neutrino interactions models [8]. Large errors in cross-section measurements and disagreements between experiments lead to systematic uncertainties in oscillation measurements. However, another possible source could come from a poor knowledge of the neutrino flux when calculating the cross-sections.

The MINERvA experiment is dedicated to study $\nu_{\mu}$ and $\bar{\nu}_{\mu}$ cross-sections for neutrinos in the 1-20 GeV range with high statistics for inclusive and exclusive channels and with interactions on several nuclear targets $\left(\mathrm{C}, \mathrm{Pb}, \mathrm{Fe}, \mathrm{H}_{2} \mathrm{O}\right.$ and $\mathrm{He}$ ) to investigate nuclear effects.

MINERvA is located on-axis in the NuMI beamline in the MINOS Target Hall and it is designed to have a good reconstruction of the neutrino interactions [9]. It consists of different nuclear targets that are followed by a core of scintillator strips (called the tracker) and surrounded by electromagnetic and hadronic calorimeters. One of the main challenges comes from the uncertainty in the neutrino flux.

A neutrino cross-section is calculated according to the Equation 1.12 as the reconstructed neutrino interactions divided by the neutrino flux and the number of target nuclei $(\mathrm{T})$. Any uncertainty in the flux enters directly to the cross-section calculation. This is the reason why knowing the flux is crucial for MINERvA. 


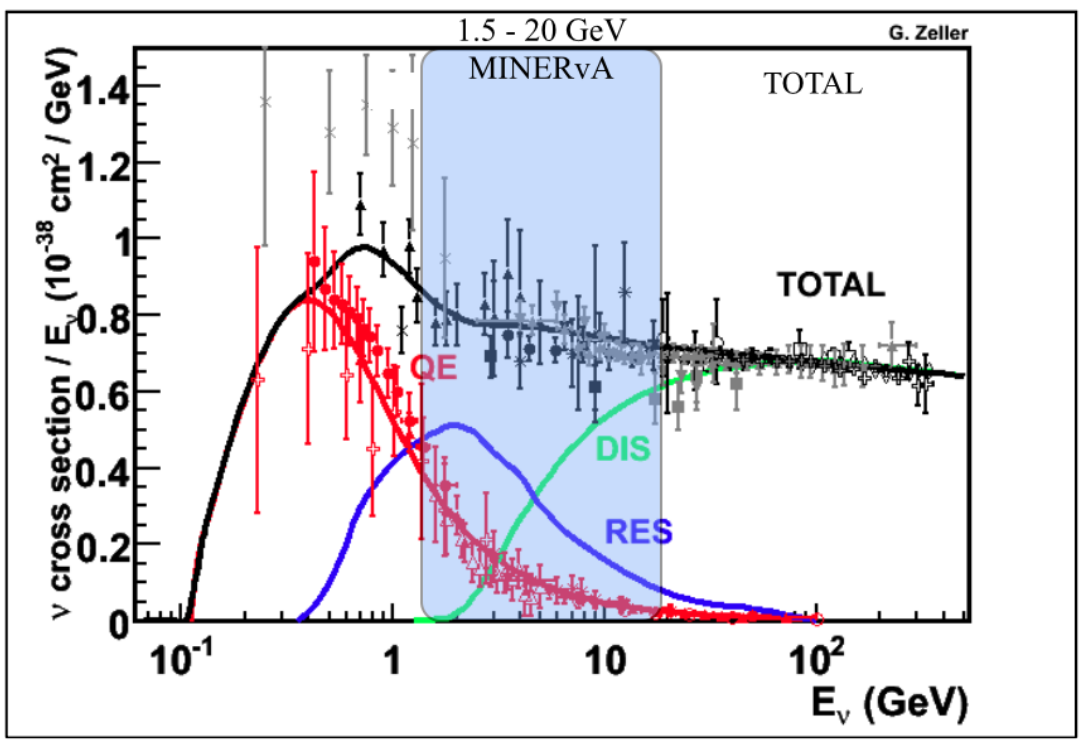

(a) $\nu_{\mu}$

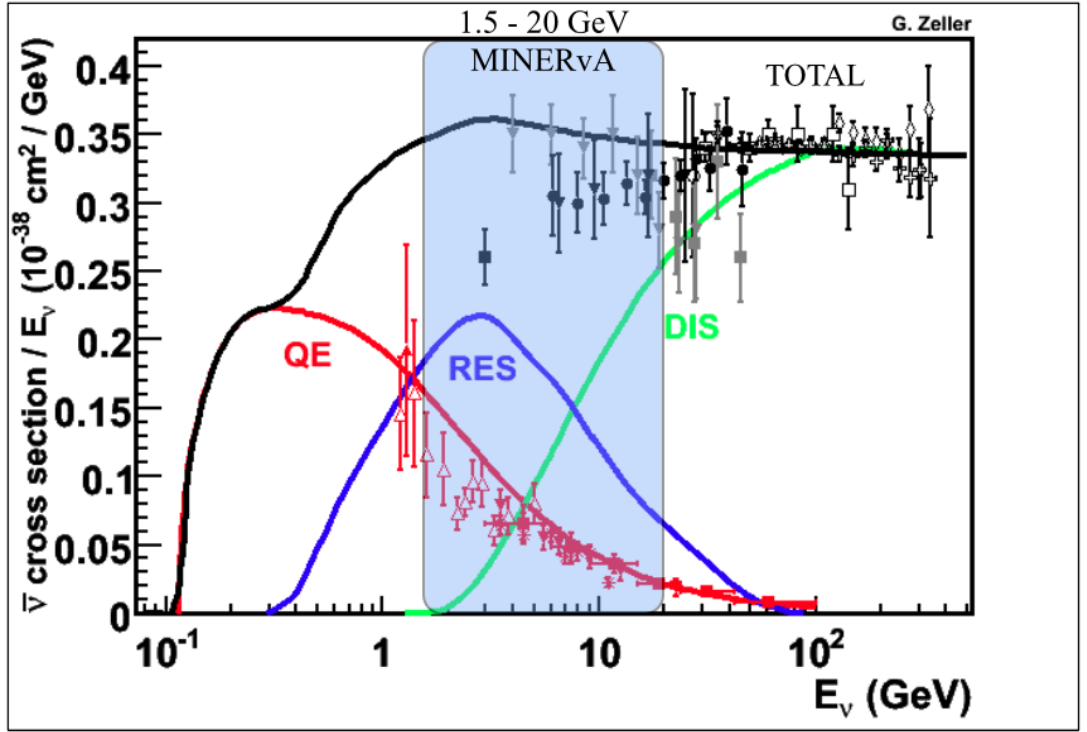

(b) $\bar{\nu}_{\mu}$

FIG. 1.4: $\nu_{\mu}$ and $\bar{\nu}_{\mu}$ charged current cross-sections per nucleon. Plots taken from [6] where the list of datasets used can also be found. 


$$
\sigma(E)=\frac{N(E)}{\phi(E) \times T}
$$

As an example of this challenge, Figure 1.5 shows the coherent charged pion production cross-section in $\nu_{\mu}$ interactions recently published by MINERvA [10]. These are the first high statistics measurements made of this channel. The uncertainties are dominated by the flux, limiting the precision of the measurement (see Figure 1.5b). This result is very important for the neutrino physics community since the neutral current analog of this channel is a potential source of background in oscillation experiments.

This thesis is about determining the NuMI flux for the MINERvA experiment by developing a procedure and tools that can be used to determine the flux for any detector that sees neutrinos from NuMI. The structure of the thesis includes a description of the NuMI beamline geometry and capabilities (Chapter 2), a description of our multi-layer approach (Chapter 3) and the hadron production correction (Chapter 4) and an analysis of our results (Chapters 5 - 7). 


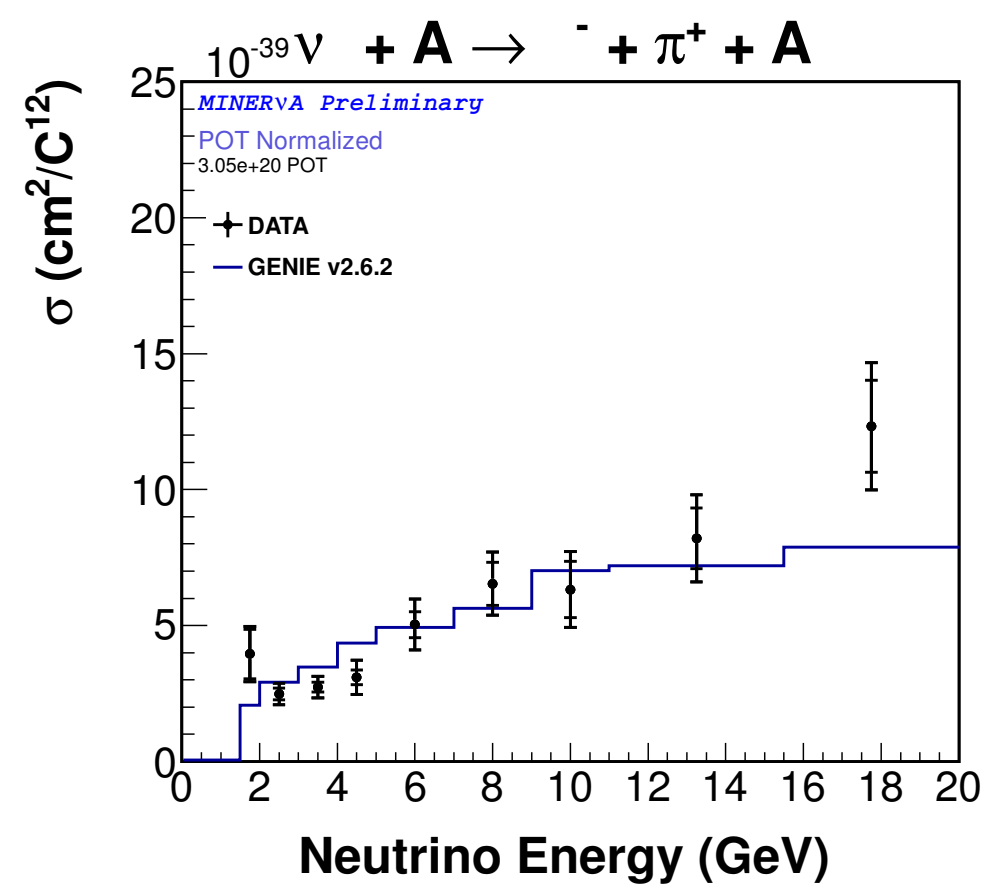

(a) Cross-section

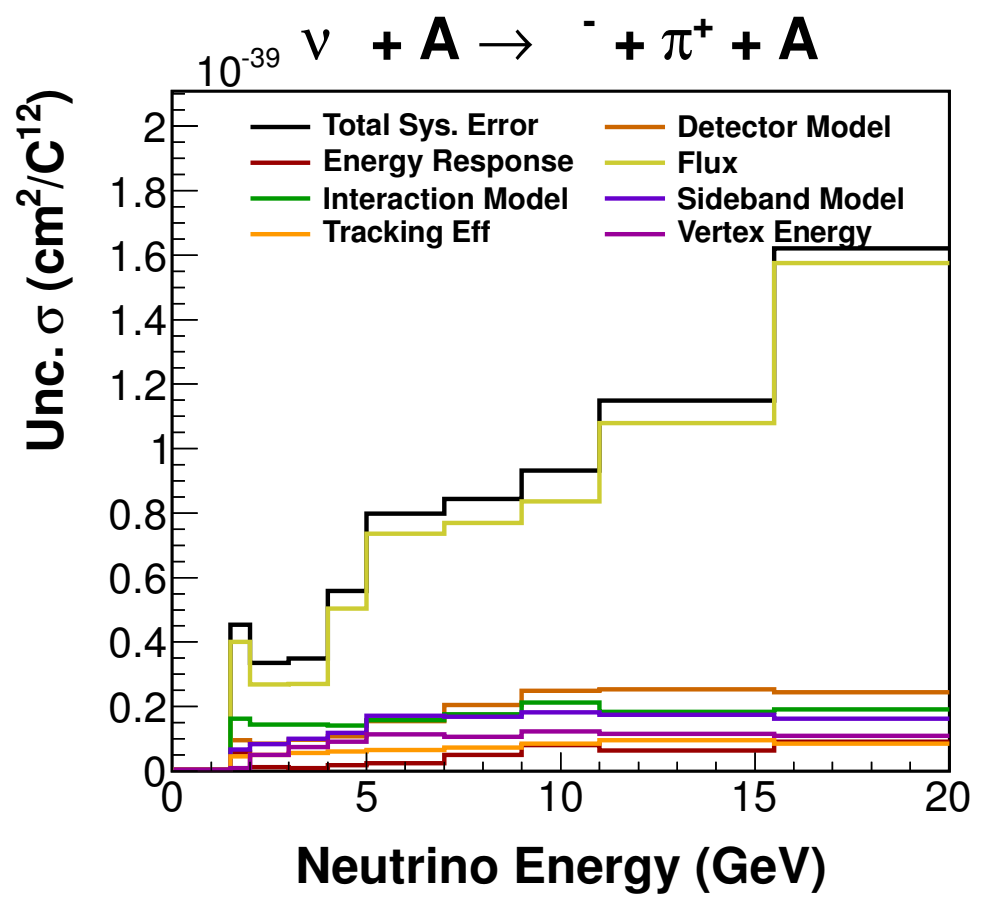

(b) Systematic uncertainties

FIG. 1.5: Coherent production of $\pi^{ \pm}$in $\nu_{\mu}$. Plots taken from [10]. 


\section{CHAPTER 2}

\section{NuMI beamline}

I start this chapter introducing the aspects of the design and construction of conventional neutrino beams as a general context to describe the NuMI beamline. Not all parts of NuMI are included here, just the relevant components for understanding its geometry and capabilities in the context of this thesis. The major challenges that are presented in detail are the focusing system and the hadronic cascade from the primary proton beam to the weak decay producing a neutrino in the beamline. All plots are based on direct output of the simulation, i.e., no correction is applied to the hadronic model. The MINERvA strategy to correct the hadron production mismodeling is presented in the Chapter 3.

\subsection{Conventional neutrino beams}

The basic concept of conventional neutrino beams is to extract a very intense proton beam from an accelerator and collide it with a target to produce short-lived particles, like pions and kaons, that will eventually decay to neutrinos. The targets are thick (a few interaction lengths long) but narrow enough to allow the produced particles to escape without interacting too many times. The particles produced have 
a boost in the direction of the beamline but in order to enhance the neutrino beam, one or more Van der Meer's magnetic horns [11, 12] are placed to focus the mesons. After the horns, the particles enter a long vacuum or low density pipe where most of mesons decay. The remaining muon and hadrons beams are removed by absorbers.

The main decay modes that produce neutrinos are presented in Table 2.1. Charged pions and charged kaons create predominantly muon neutrinos and a small component of electron neutrinos. Muons can also be generated in these decays and contribute to the electron neutrino flux, but these are highly suppressed because they are mostly absorbed before they decay.

\begin{tabular}{|c|l|c|}
\hline Decay & Chanel & Branching ratio (\%) \\
\hline 1 & $\pi^{ \pm} \rightarrow \mu^{ \pm}+\nu_{\mu}\left(\bar{\nu}_{\mu}\right)$ & 99.9877 \\
2 & $\pi^{ \pm} \rightarrow e^{ \pm}+\nu_{e}\left(\bar{\nu}_{e}\right)$ & 0.0123 \\
\hline \hline 3 & $K^{ \pm} \rightarrow \mu^{ \pm}+\nu_{\mu}\left(\bar{\nu}_{\mu}\right)$ & 63.55 \\
4 & $K^{ \pm} \rightarrow \pi^{0}+e^{ \pm}+\nu_{e}\left(\bar{\nu}_{e}\right)$ & 5.07 \\
5 & $K^{ \pm} \rightarrow \pi^{0}+\mu^{ \pm}+\nu_{\mu}\left(\bar{\nu}_{\mu}\right)$ & 3.353 \\
\hline \hline 6 & $K_{L}^{0} \rightarrow \pi^{ \pm}+e^{\mp}+\nu_{e}$ & 40.55 \\
7 & $K_{L}^{0} \rightarrow \pi^{ \pm}+\mu^{\mp}+\nu_{\mu}$ & 27.04 \\
\hline \hline 8 & $\mu^{ \pm} \rightarrow e^{ \pm}+\nu_{e}\left(\bar{\nu}_{e}\right)+\bar{\nu}_{\mu}\left(\nu_{\mu}\right)$ & 100.0 \\
\hline
\end{tabular}

TABLE 2.1: Main decay modes and their branching ratios of charged pion, charged kaons, neutral kaons and muons to neutrinos.

Considering a two-body decay (like in decay types 1-3 at the Table 2.1), we can calculate the energy of the neutrino, assuming that they are moving in a near forward direction, as a function of its meson parents (Equation 2.1). Muon and meson masses are represented by $m_{\mu}$ and $M$, respectively, the angle of the neutrino flux with respect to the pion parent momentum is $\theta_{\nu}$, and the Lorentz factor $\gamma=E_{\pi(K)} / M$ :

$$
E_{\nu} \approx \frac{\left(1-\frac{m_{\mu}^{2}}{M^{2}}\right) E_{\pi(K)}}{1+\gamma^{2} \tan ^{2} \theta_{\nu}}
$$


For detectors located on the beam axis (on-axis), like MINERvA and MINOS, the angle $\theta_{\nu}$ is zero. Then the energies of the neutrino and its parent have a linear relation, $E_{\nu} \approx 0.43 E_{\pi}$ and $E_{\nu} \approx 0.95 E_{K}$ for pion and kaon parents, respectively. For off-axis experiments like NOvA, where $\theta_{\nu} \approx 12.26 \mathrm{mrad}$, the relation becomes non-linear due to the energy dependency of the Lorentz factor. Figure 2.1 shows the functional form of the Equation 2.1 for pion neutrino parents at three positions: on-axis detectors (black line), the center of the front face of NOvA Near detector (red line) and the center of MicroBooNE (in purple). For practical reasons, the horizontal axis is cut at $10 \mathrm{GeV}$, but all off-axis curves reach a maximum and then decrease their value monotonically.

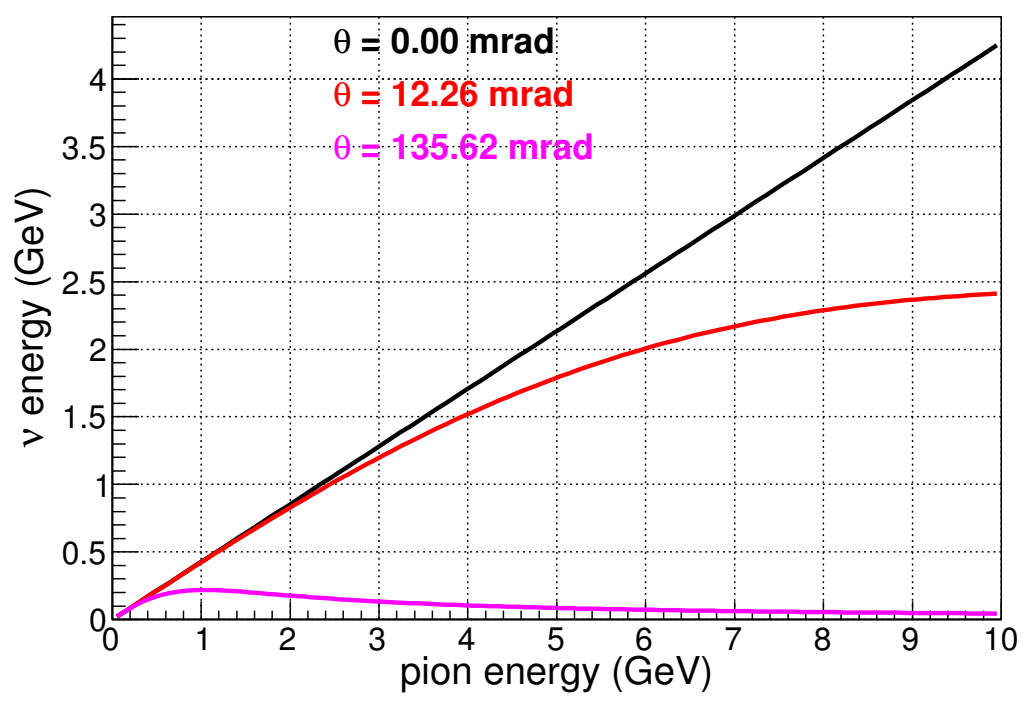

FIG. 2.1: Functional form given in Equation 2.1 for three positions in NuMI beamline: on-axis (MINERvA and MINOS) in black, off-axis (NOvA and MicroBooNE).

Some criteria when designing a conventional neutrino beam include:

- The proton beam. Depending on the physics goals, selecting the energy and intensity of the primary proton beam one should follow two rules of thumb: firstly, 
higher energy proton beams produce higher energy mesons and then more energetic neutrino fluxes. Secondly, pion and kaon multiplicities are approximately proportional to the number of protons colliding on the target (POT) times the proton energy (referred as a "proton power").

- Target. Increasing the target length has the benefit to generate more interactions of the primary protons, however, it can bring two undesirable effects: it increased the scattering of the produced mesons and, in conjunction with an intense proton beam, generates more interactions and a hotter target.

One way to have a cooler target is by making it wider, but more material budget will produce more scattering. With these considerations, targets are constructed in small segments made of low Z materials (like carbon, beryllium or aluminum) to dissipate the heat more easily. A cooling system is sometimes needed, depending on beam power.

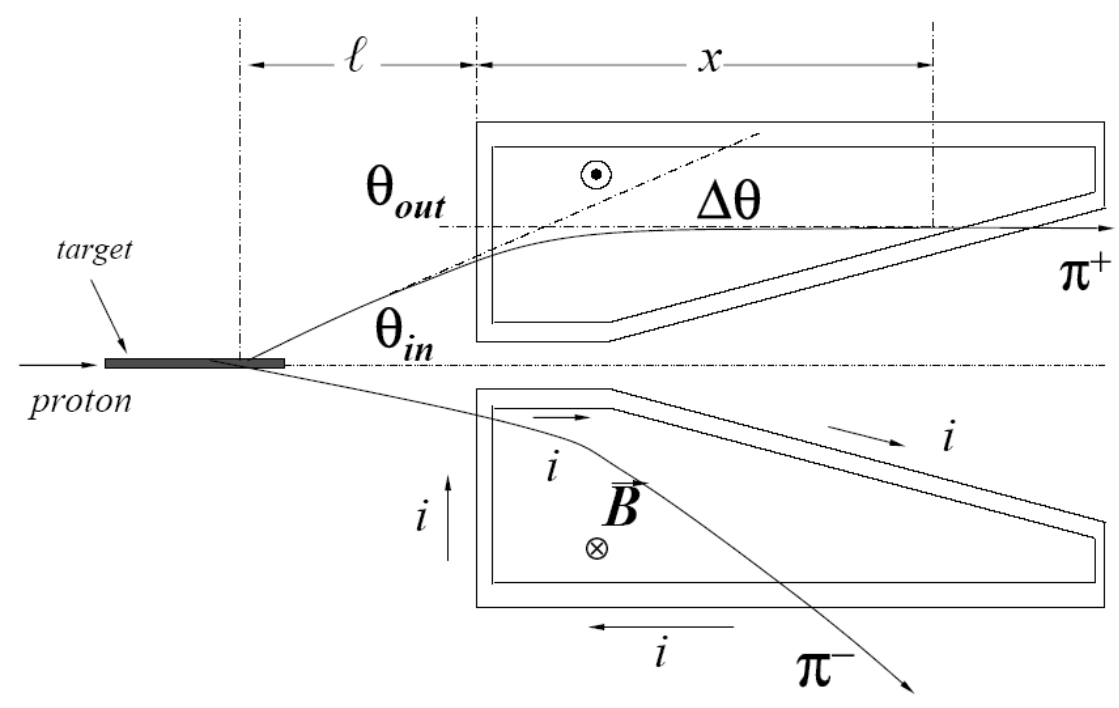

FIG. 2.2: Diagram of a target and a single conical magnetic horn. Plot taken from [11].

- Focusing system. The challenge in focusing is to cancel the transverse momen- 
tum $\left(p_{T}\right)$ of as many hadrons that exit the target as possible ${ }^{1}$. This means that higher $p_{T}$ hadrons have to travel more distance in the magnetic horns and, at the same time, the interaction probability in the horns has to be minimized. Figure 2.2 displays the particle trajectories of pions entering to a horn. In this case, $\pi^{+}$ are focused and $\pi^{-}$are deflected due to direction of the magnetic field.

Modern magnetic horns are composed of 2-layered thin coaxial sheet conductor with a volume between the layers filled with low-density gas (air, helium or argon). The current circulates in one direction in the inner layer and in the opposite direction in the outer layer generating a toroidal magnetic field between layers that falls as $1 / R$ by Amperes law and has zero value outside the horn. Depending on the direction of the current (and the magnetic field) the horns focus charged particles with a particular sign and deflect particles with the opposite sign. In this way, the wanted neutrino type is enhanced while the undesired neutrino type (background) is minimized.

The sketch in the left lower part of Figure 2.7 shows this characteristic in the context of NuMI description in the next section. Furthermore, having a parabolic front face makes the total distance travelled by the particle in the horn (and the total momentum change) proportional to $p_{T}$.

- Decay Pipe. Increasing the length of the decay pipe allows more and also higher pions to decay but makes fewer muons reach the absorbers and the number of electron neutrinos increase. The final length depends on the required neutrino energy and how much $\nu_{e}$ contamination is tolerable according the the physics goals of the experiment. Ideally, the pipe should be evacuated to eliminate any interactions but this requires more material in the windows of the pipe to keep the vacuum. There are always concerns of catastrophic failures in the system too.

\footnotetext{
${ }^{1}$ Calculations indicate that it increases the neutrino flux by a factor of 25 approximately [11].
} 
Pipes filled with helium or air reduces the neutrino spectrum by a few percent and are good alternatives.

\section{$2.2 \quad$ NuMI description}

The NuMI design was motivated by neutrino oscillation studies ([13], [14]). The parameters of the main components have been optimized to meet the objectives of MINOS for the Low Energy (LE) run and NOvA for the Medium Energy (ME) run. Its high intensity neutrino flux has been used by other experiments like MINERvA to study cross-sections.

The NuMI neutrino beamline starts at Fermilab, Illinois, and travels through the crust of the Earth towards the Soudan Mine in Minnesota and then exits the Earth. In its travel, $1.04 \mathrm{~km}$ from the starting point, it encounters the MINOS Near Detector and MINERvA, and at $734 \mathrm{~km}$ the MINOS Far Detector.

A schematic view of the NuMI beamline is shown in Figure 2.3. A detailed description of every part can be found in Ref. [13]. In this section, I will be focused on those components related to this thesis: the primary beam, target, magnetic horns and the decay pipe.

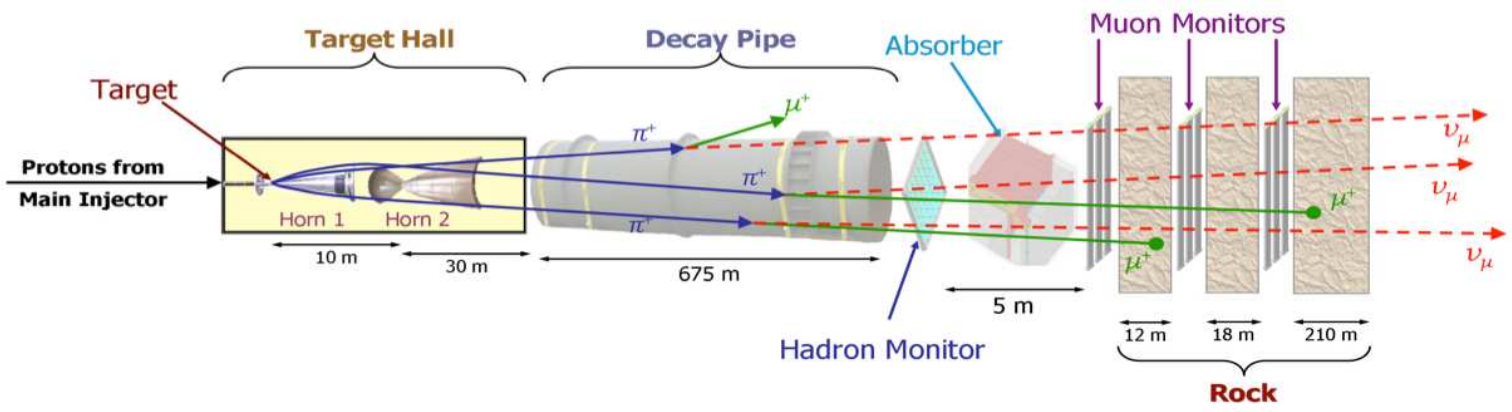

FIG. 2.3: Schematic view of NuMI beamline. Taken from [13]. 


\subsubsection{Primary proton beam}

The proton accelerator at Fermilab starts with $H^{-}$ions being accelerated to $400 \mathrm{MeV}$ kinetic energy in the LINAC (LINear ACcelerator [15]). Those ions are converted to $H^{+}$(protons) when they pass by a carbon foil and injected to the Booster synchrotron ([16]), where the protons are accelerated to $8 \mathrm{GeV}$ kinetic energy. Some of these protons are used for the short baseline neutrino experiments before entering to the Main Injector (MI).

The MI ring is a synchrotron accelerator of $3.3 \mathrm{~km}$ circumference that, in turn, accelerates the protons to $120 \mathrm{GeV}$, which are then used for many purposes. It served as the proton (and antiproton ${ }^{2}$ ) source to the Tevatron and provides beamlines for fixed target experiments and test beams, and it is also used to create neutrinos. Protons from NuMI are extracted in 6 batches $^{3}$ in approximately 10 ps in a "single turn" 4 and bent 58 mrad downward towards the MINOS Near detector. Figure 2.4 shows a plan and elevation view of the NuMI Beam Facility. The beam is directed to the Target Hall, $41 \mathrm{~m}$ underground, that contains the target and the magnetic horns.

The beam spot size at the target is gaussian with $\sigma_{x}=1.1 \mathrm{~mm}$ and $\sigma_{y}=$ $1.2 \mathrm{~mm}$. In the LE run, the cycle time was between $2.1 \mathrm{~s}$ and $2.4 \mathrm{~s}$ per spill with $2.2 \times 10^{13} \mathrm{POT} / \mathrm{spill}$ in 2005 rising to $3.6 \times 10^{13} \mathrm{POT} / \mathrm{spill}$ in 2012 . The average beam power was $\sim 250 \mathrm{~kW}$. For the ME run, an upgrade is currently in progress to reach $700 \mathrm{~kW}$ beam power for NOvA physics goals. At the time of writing, the power is already $\sim 400 \mathrm{~kW}$ with a $1.3 \mathrm{~s}$ cycle time. The beam spot is wider than in the LE run with $\sigma_{x(y)}=1.3 \mathrm{~mm}$ RMS.

\footnotetext{
${ }^{2}$ The Main Injector provided protons to the antiproton accumulator up to 2011 when Tevatron ceased operations.

${ }^{3}$ During the Tevatron era, 5 batches were destined to NuMI and 1 for the antiproton accumulator for Tevatron and the NuMI extraction time was $8 \mu \mathrm{s}$.

4 "Single turn" technique is a fast deliver of the entire beam in a single extraction[17].
} 


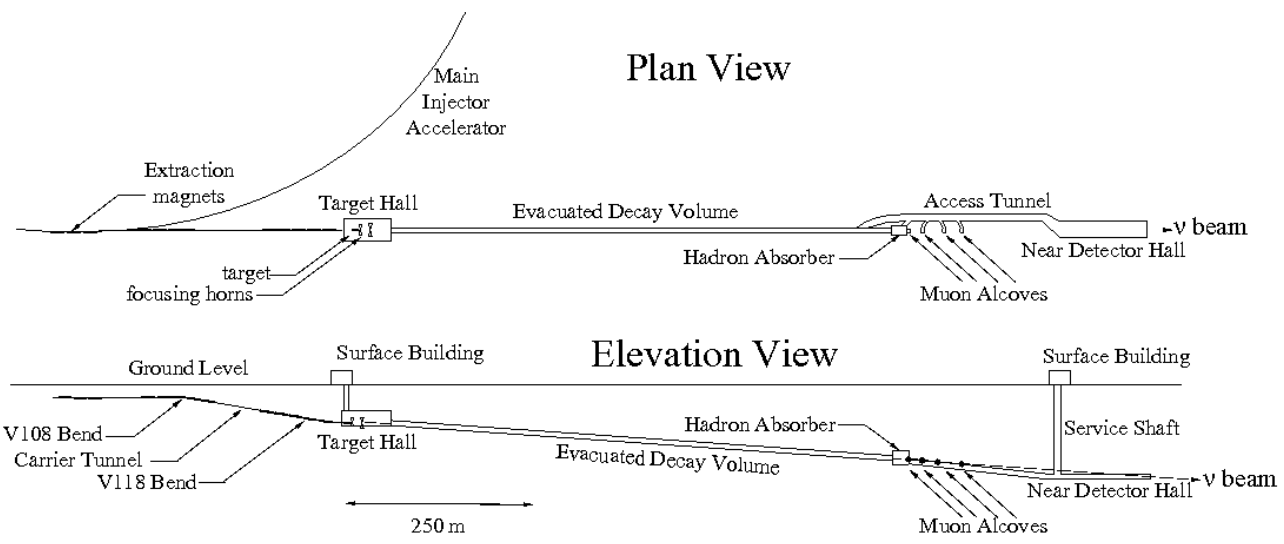

FIG. 2.4: Plan elevation view of NuMI Beam Facility. Taken from [13].

\subsubsection{Target}

The target is a rectangular graphite rod with $1.78 \mathrm{~g} / \mathrm{cm}^{3}$ density and it is segmented in rectangular pieces (called "fins") rounded at the edges and stacked along the beam direction with a small space between each. The target is cooled by water that circulates in a pipe bonded to the upper and lower edge of each fin. The whole target is enclosed in a helium filled container. Budal monitors are placed upstream to check the position of the target by scanning the beam in the horizontal or vertical direction ${ }^{5}$.

A picture of the LE target can be seen in Figure 2.5.

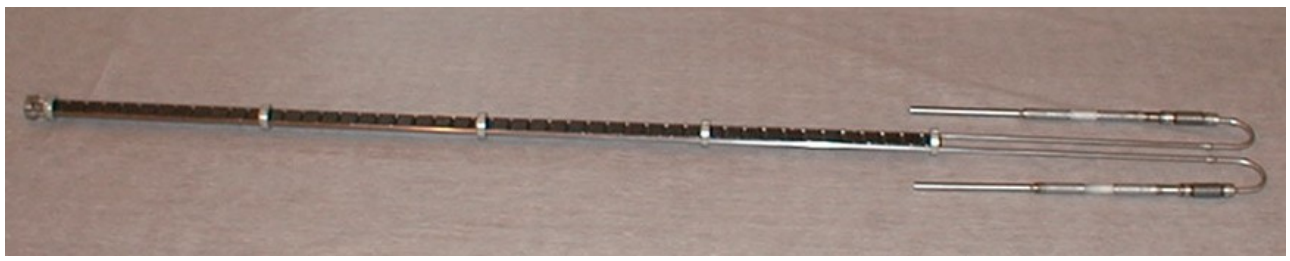

FIG. 2.5: Photograph of the LE NuMI target. Taken from [19].

\footnotetext{
${ }^{5}$ Budal Monitors measure the signal from electrons and other particles that are kicked off when the proton beam interacts in a fin. They provide a position dependent signal proportional to the beam intensity. The original proposal can be found in [18].
} 
A sketch of the target is shown in Figure 2.6. At the top, a longitudinal view of a couple of fins where $\mathrm{X}, \mathrm{Y}$ and $\mathrm{Z}$ represent the distance between fins, the fin length and the length of the rounded side is shown. At the bottom, a cross-sectional view of the LE (left) and ME (right) targets and their dimensions is showed. The blue circle indicates the incident point of the proton beam.

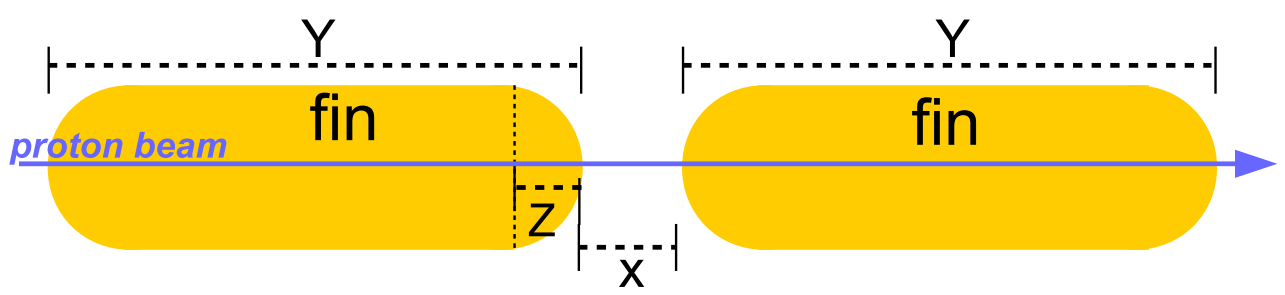

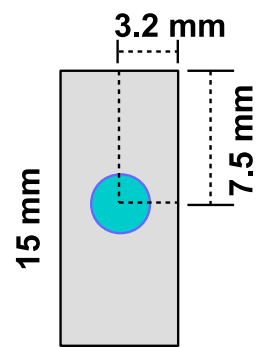

$6.4 \mathrm{~mm}$

LE target

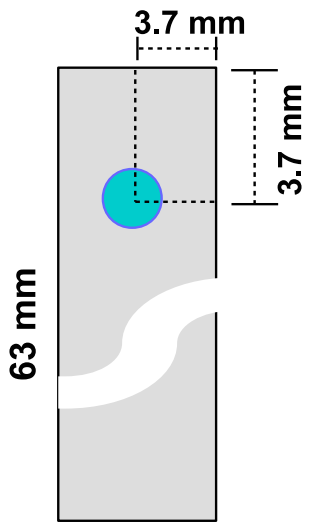

$7.4 \mathrm{~mm}$ ME target

FIG. 2.6: Sketch of the LE and ME targets.

For the LE run, the target consists of 47 vertical fins plus 1 additional horizontal fin with a Budal monitor ${ }^{6}$. Each fin is $20 \mathrm{~mm}$ long (Y), $15 \mathrm{~mm}$ tall and $6.4 \mathrm{~mm}$ wide. The space between fins is $0.3 \mathrm{~mm}(\mathrm{X})$. The beam is centered exactly in the center of the transverse view of the target. The Budal monitor is placed $167.2 \mathrm{~mm}$ upstream of the target for horizontal scanning.

\footnotetext{
${ }^{6}$ The system fin with a Budal monitor is simply called "Budal Monitor" (BM) in the context of this thesis, unless otherwise indicated.
} 
For the ME run, the target consists of 48 vertical fins plus 2 additional fins with Budal monitors, one vertical and the other horizontal. Due to the higher beam power, ME fins are larger than the LE ones. Each fin is $24 \mathrm{~mm}$ long (Y), $63 \mathrm{~mm}$ tall and $7.4 \mathrm{~mm}$ wide. The separation between fins is $0.5 \mathrm{~mm}(\mathrm{X})$. The location of the beam is at the center of the horizontal side but $3.7 \mathrm{~mm}$ from the top of the target. The Budal monitors have the same dimensions as the fins: the vertical one, for vertical scanning, is located $28.5 \mathrm{~mm}$ from the target, while the horizontal one, for horizontal scanning, is placed $57.5 \mathrm{~mm}$ from the target.

A $1.5 \mathrm{~m}$ long baffle protects the target and the horns (especially the neck of the horn) from any missteering of the beam and it helps monitor the beam. It consists of a $57 \mathrm{~mm}$ diameter graphite core with a $11 \mathrm{~mm}$ diameter hole, all encased in an aluminum tube and two $0.5 \mathrm{~mm}$ thickness beryllium windows. The target, Budal monitors and baffle are mounted in the same carrier. This allows them to be moved together and to control the distance with respect to the horn when the beam configuration changes.

Table 2.2 summarizes the main differences between the target in LE and ME configurations.

\begin{tabular}{|l|c|c|}
\hline & LE & ME \\
\hline Cross Sectional view & $6.4 \times 15 \mathrm{~mm}^{2}$ & $7.4 \times 63 \mathrm{~mm}^{2}$ \\
\hline Segment length & $20 \mathrm{~mm}$ & $24 \mathrm{~mm}$ \\
\hline "Fins" & $47+1 \mathrm{BMs}$ & $48+2 \mathrm{BMs}$ \\
\hline Total Length & $960 \mathrm{~mm}(\sim 2 \lambda)$ & $1200 \mathrm{~mm}(\sim 2.5 \lambda)$ \\
\hline
\end{tabular}

TABLE 2.2: Summary of LE and ME target parameters. "BM"s are Budal Monitors (see text). 


\subsubsection{Magnetic horns}

NuMI uses two magnetic horns, each one $3 \mathrm{~m}$ long. A current circulates though the inner conductor (IC) and returns by the outer conductor (OC) creating a toroidal magnetic field between them. The conductors are made of aluminum and the field region is filled with Argon. Figure 2.7 shows a not-to-scale sketch of the crosssectional view of Horn 1, indicating the direction of the current and the magnetic field affecting the particles that enter the horn from the target. The picture in Figure 2.7 is a photograph of the real Horn 1 Inner Conductor in the welding machine before being placed in the Target Hall.

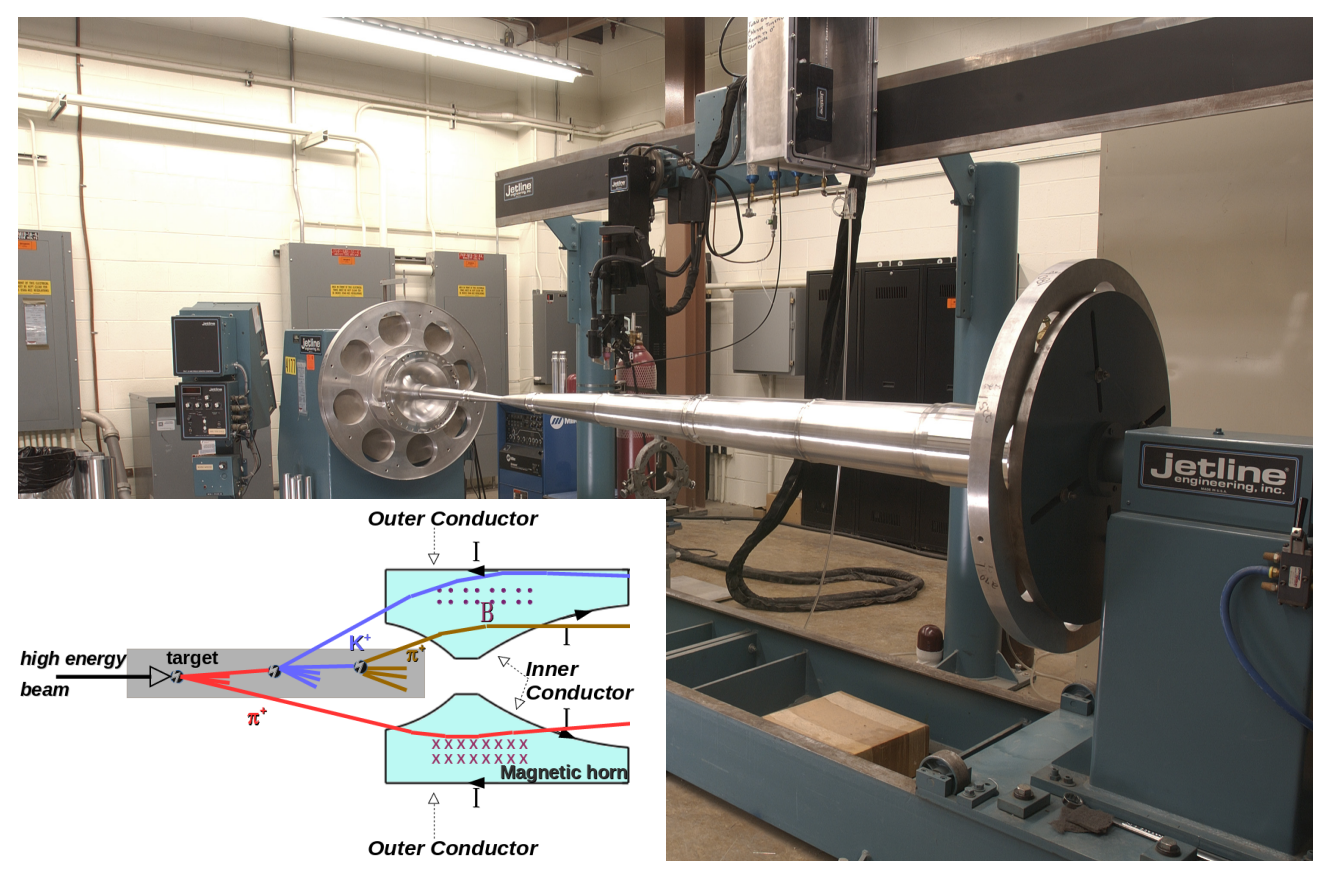

FIG. 2.7: Photograph of the Horn 1 in the welding machine before being placed in the Target Hall. In the bottom left corner, a sketch of the target and Horn 1 indicating some possible particle trajectories. Photograph taken from [19].

The IC surface is made of two parabolic surfaces of rotation, one upstream and 
the other downstream, welded together with a short cylindrical neck. The thicknesses of the parabolic parts are $2 \mathrm{~mm}$ and $3 \mathrm{~mm}$ for Horn 1 and Horn 2 respectively, and the neck is $4.5 \mathrm{~mm}$ thick. The OC diameter for Horn 1 is $\sim 35 \mathrm{~cm}$ and for Horn 2 it is $\sim 79 \mathrm{~cm}$, and they are $25.4 \mathrm{~mm}$ thick.

The current is pulsed in a half-sine wave with typical peak values $182.1 \mathrm{kA}$ and $200 \mathrm{kA}$ for LE and ME, respectively. The duration of the pulse is $2.3 \mathrm{~ms}$ and it is long enough to provide a stable magnetic field during the $10 \mu$ s spill. The inside surfaces of the ICs are continuously sprayed with cooling water to remove heat.

A map of the magnetic field in the Horn 1 is shown in Figure 2.8a with respect to the beamline direction $(\mathrm{Z})$ and the vertical position $(\mathrm{Y})$ in the simulation coordinate system. The NuMI simulation will be explained in Section 2.2.5. Due to the rotational symmetry of the horns, the $\mathrm{Y}$ axis can be replaced by a radial axis. The dashed line represents the target in the nominal position for LE (more details about the target's longitudinal position will be given in Section 2.3). The maximum magnetic field is around $2.7 \mathrm{~T}$ in the neck region, necessitating a thicker conductor. The field decreases as $1 / \mathrm{r}$ as can be seen in the radial distribution in Figure 2.8b.

The relative distances between the horns and between the Horn 1 and the target are crucial to the focusing process. Horn 2 is located downstream from Horn 1 by $7 \mathrm{~m}$ and $16 \mathrm{~m}$ approximately in LE and ME, respectively. Also, in the ME configuration, the target is pulled back from the Horn 1. The effect that this causes in changing the beam energy is explained in Section 2.3.

One way to understand the effect of the focusing system is by splitting the neutrino flux spectrum into categories (called "focusing components") with respect to how the neutrino parent meson travels from the target through the horns (as displayed in Figure 2.9), and it depends on the absolute momentum and the relative value of the transverse momentum of the mesons with respect to their longitudinal momentum. These categories are: 


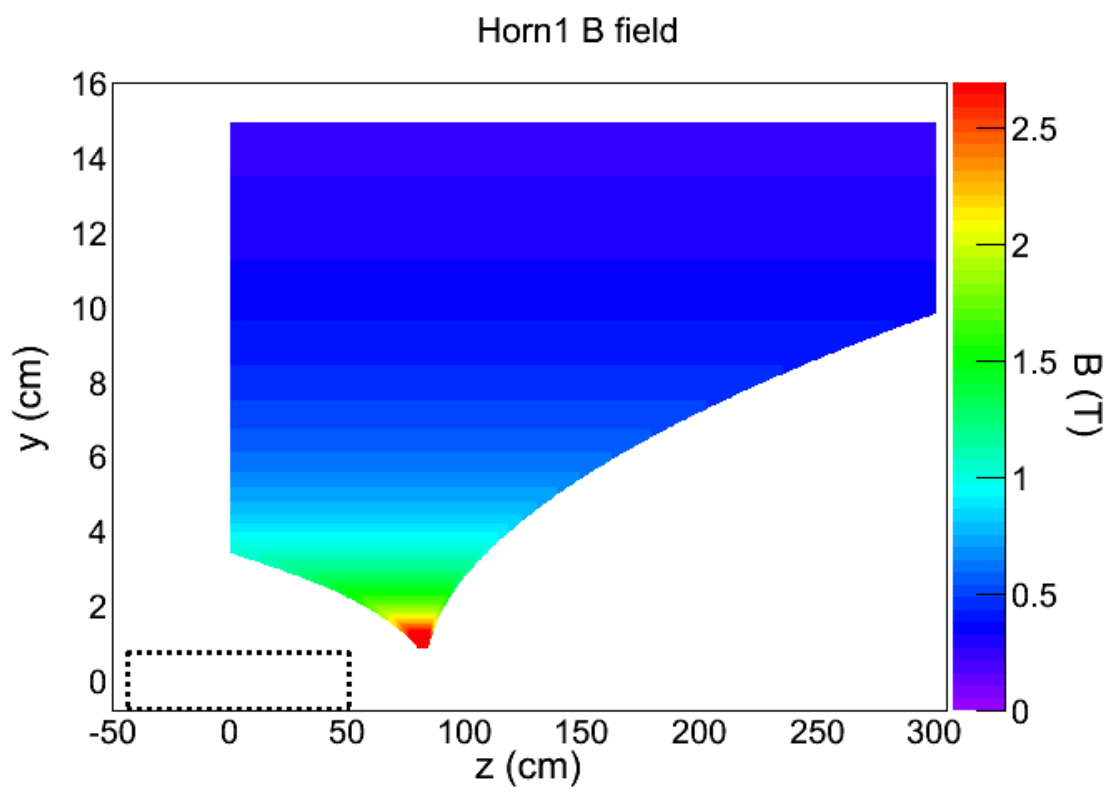

(a) Longitudinal cross sectional distribution (the target in LE010z position configuration is drawn in dashed line as reference).

Horn1 B field

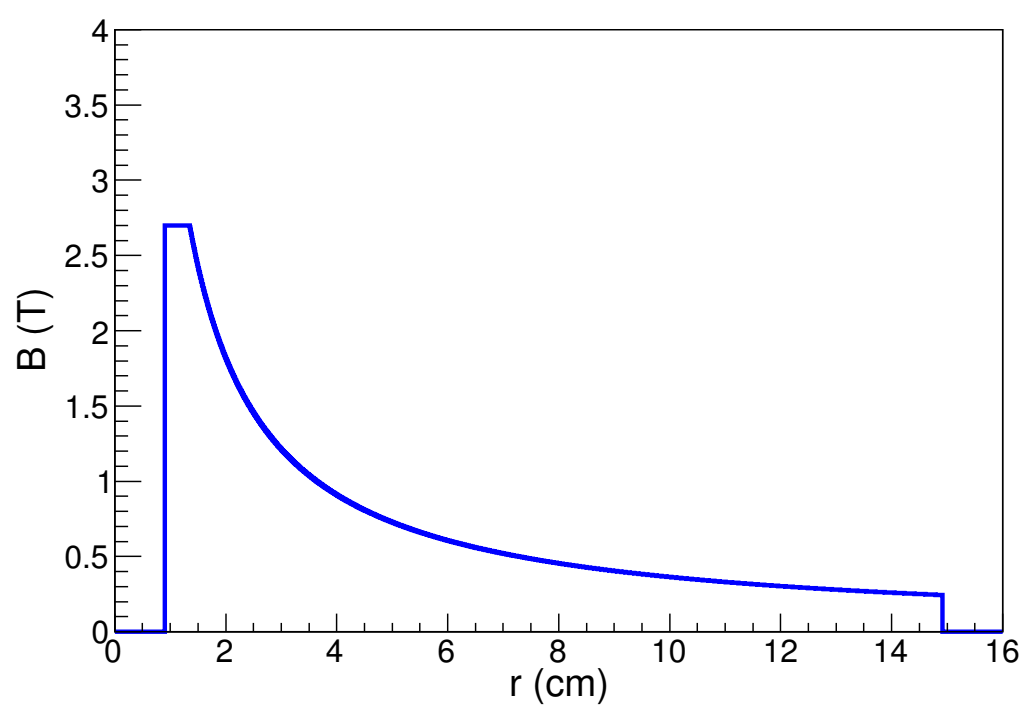

(b) Radial distribution.

FIG. 2.8: Horn 1 magnetic field for $185 \mathrm{i}$ current configuration (182.1 kA). 
- Unfocused: mesons that pass through the necks of both horns (solid red line). They have high momentum (typically more than $15 \mathrm{GeV} / \mathrm{c}$ ) and very small $p_{T}$ $\left(p_{T}<0.1 \mathrm{GeV}\right)$.

- Horn2-only: mesons that pass through the neck of the first horn and cross the Horn 2 (dashed red line). They have an intermediate energy (typically between 9-15 $\mathrm{GeV} / \mathrm{c}$ ) and the transverse momentum creates a small deviation from the beamline.

- Underfocused: mesons that pass through both horns receiving a correction in Horn 1 and complemented by Horn 2 (solid orange line). The momentum of these particles in the LE mode, is in $5-15 \mathrm{GeV} / \mathrm{c}$ range and a $p_{T}>0.2 \mathrm{GeV} / \mathrm{c}$.

- Horn1-only: mesons that are affected only by the Horn 1 and not by the Horn 2. They have $p_{T}>0.2 \mathrm{GeV} / \mathrm{c}$ as in underfocused category but less momentum making the Horn 1 bending the particle to the Horn 2 neck. Mesons in this category decay into neutrinos early.

- Overfocused: mesons with low momentum (typically less than $5 \mathrm{GeV}$ in $\mathrm{LE}$ configuration) that are overcorrected by the Horn 1 and that are also corrected by the Horn 2 .

In the Appendix A, Figures A.2-A.7 show the kinematic distributions of $\pi^{+}$ neutrino parents when they exit the target per focusing component and per beam configuration.

Figure 2.10 shows the focusing components for the $\nu_{\mu}$ flux at MINERvA in the LE101z185i beam configuration (the beam configuration convention is explained in the next section). In addition, the category "others" has been added for those neutrino parents born outside of the target (dashed black line). The total LE $\nu_{\mu}$ flux that passes through MINERvA is shown as a solid black line. 


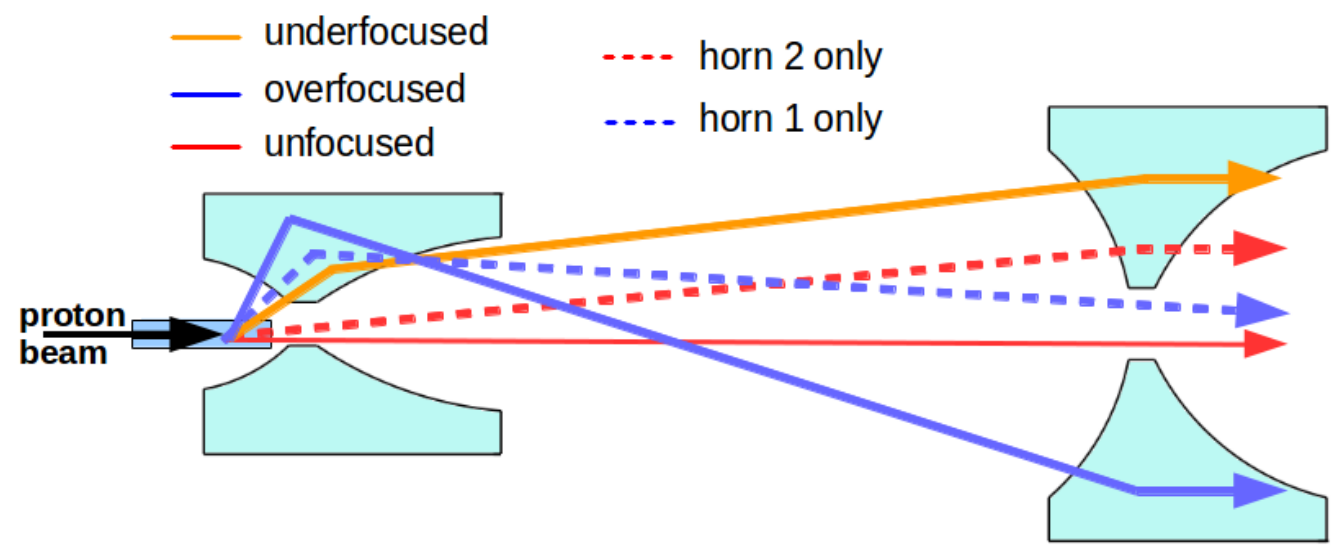

FIG. 2.9: Focusing components.

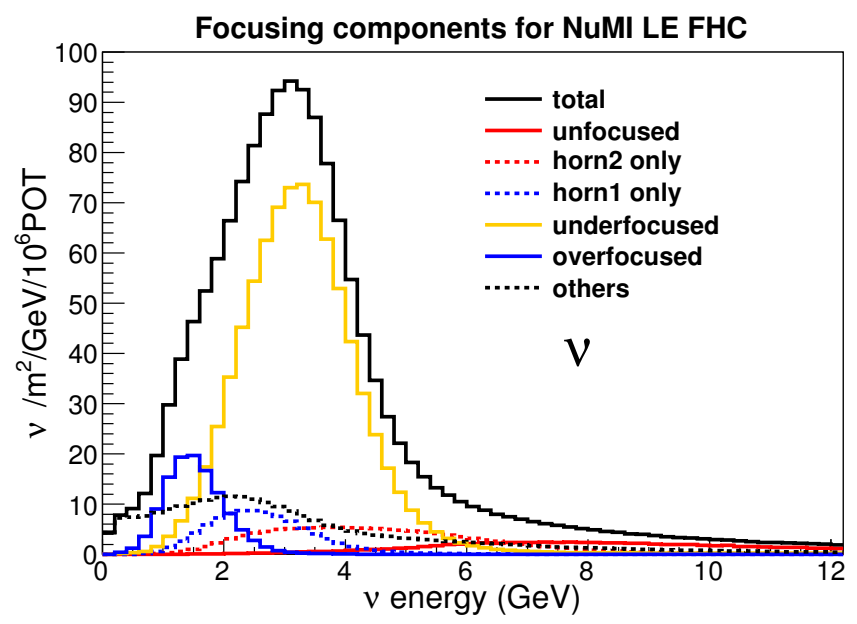

FIG. 2.10: Focusing components of $\nu_{\mu}$ flux in LE010z185i that pass through MINERvA. 
The ratios of every focusing component with respect to the part of the $\nu_{\mu}$ flux in MINERvA that has a $\pi^{+}$parent and $K^{+}$parent are shown in Figure 2.15a and b, respectively. As will be seen later in Section 2.4.1, most of the neutrinos with energy less than $20 \mathrm{GeV}$ have a $\pi^{+}$parent and greater than $20 \mathrm{GeV}$, a $K^{+}$parent.

For $\pi^{+}$we extend the neutrino energy to $20 \mathrm{GeV}$. As we expect, as we go from higher to lower momentum, unfocused, underfocused and overfocused pion decays populate the $\nu_{\mu}$ flux. A narrow momentum range values allow the pions to be focused by just one horn. The very low energy neutrinos come mostly from pions born outside of the target from secondary and tertiary hadrons.

The ratios for $K^{+}$cover up to $40 \mathrm{GeV}$. The underfocused kaons become relevant around 5-20 GeV. For energies in 20-40 GeV, the horn2-only component kaons are predominant.

The NOvA Near Detector $\nu_{\mu}$ flux spectrum and its focusing components are shown in Figure A.8 in the Appendix A.

\subsubsection{Decay pipe and Absorbers}

The Decay Pipe begins $46 \mathrm{~m}$ downstream of the NuMI Target Hall. It has a cylindrical shape with a $1 \mathrm{~m}$ inner radius, $675 \mathrm{~m}$ length, and is surrounded by a $9.5 \mathrm{~mm}$ thick wall made of iron. It is cooled by water and surrounded by poured concrete shielding. Almost all pions with energy less than $10 \mathrm{GeV}$ decay to $\nu_{\mu}$ in the Decay Pipe.

Originally the pipe was evacuated but later was filled with helium gas. The effect of adding $\mathrm{He}$ on the neutrino spectrum was of the order of $10 \%$ reduction around $3-4 \mathrm{GeV}$ and $5 \%$ increase for higher energies. In the context of this thesis, and motivated by the simulation name convention, the Decay Pipe inner volume is abbreviated as DVOL and its wall as DPIP. 


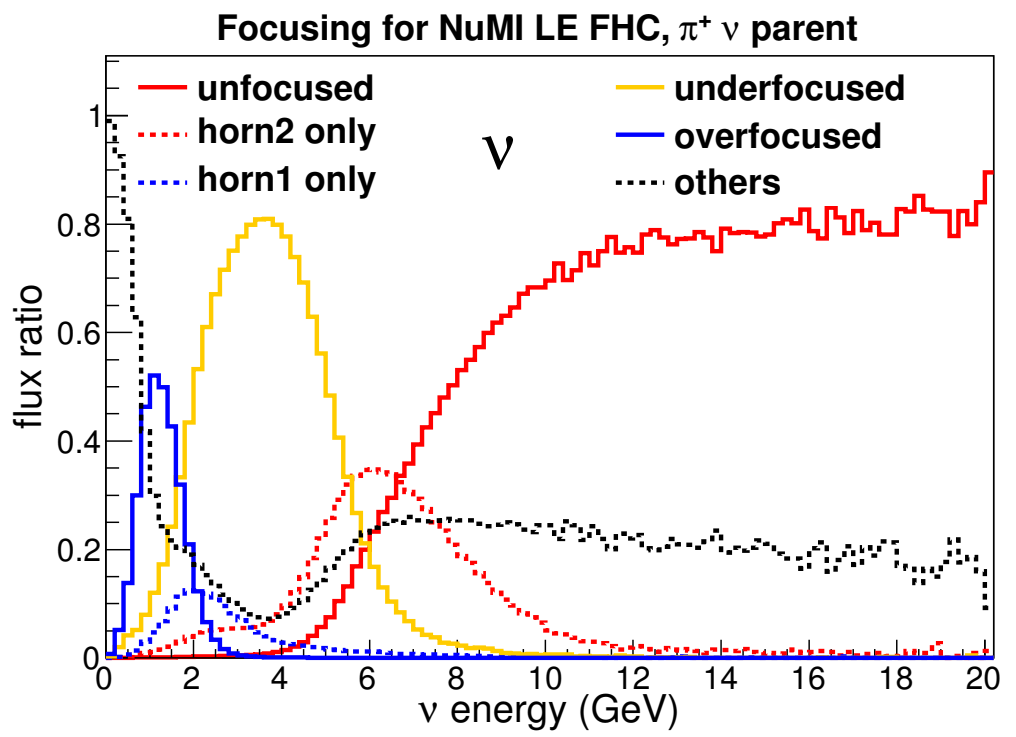

(a) $\pi^{+} \nu_{\mu}$ parent.

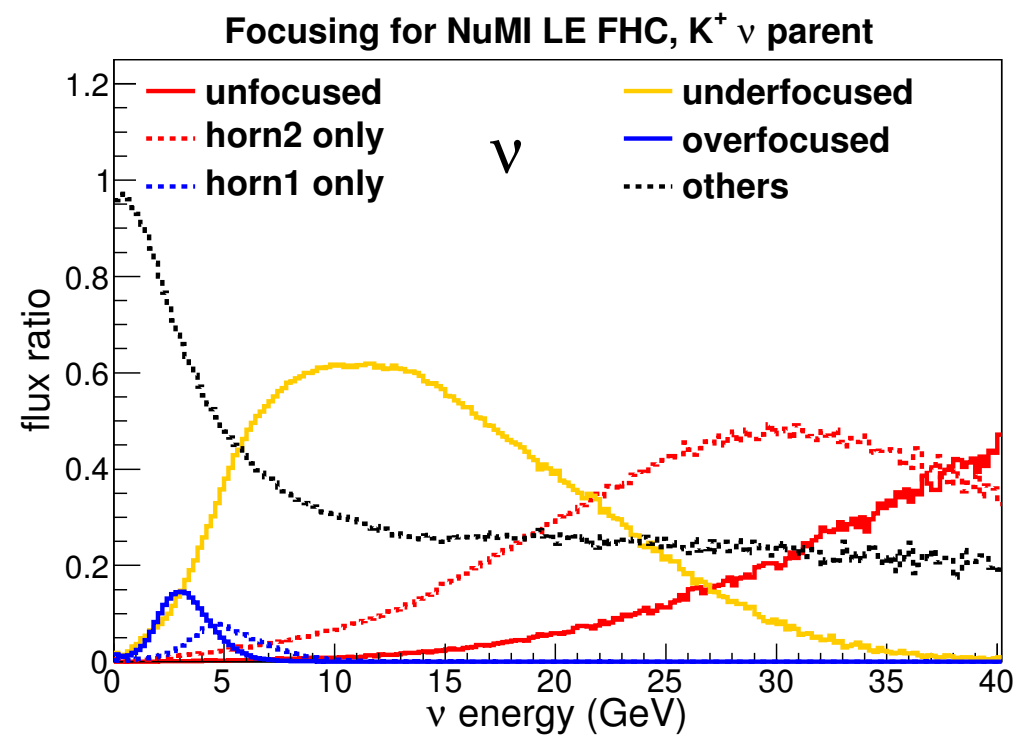

(b) $K^{+} \nu_{\mu}$ parent.

FIG. 2.11: Beam focusing component ratios of $\nu_{\mu}$ flux in the LE010z185i configuration that pass through MINERvA. 
Just downstream of the Decay Pipe a massive structure made of aluminum, steel and concrete is placed to absorb the residual hadrons still remaining in the cascade. Is is a box $5.5 \mathrm{~m}$ wide, $5.6 \mathrm{~m}$ tall, and $8.5 \mathrm{~m}$ long. Following the absorber, $240 \mathrm{~m}$ of earth, mainly dolomite rock, separates the Absorber and the MINOS Hall. The rock shields experiments in the MINOS hall from the residual $\mu$ beam.

The NuMI components that were described in this section are relevant in the context of this thesis, as will be clear in Section 2.4. Other major components, like the muon and hadron monitors, can be found in Ref. [13].

\subsubsection{NuMI simulation}

The geometry and physics associated with the NuMI beamline are implemented in an entirely GEANT4-based [20] Monte Carlo (MC) simulation called g4numi. ${ }^{7}$ The choice of GEANT4 is due its flexibility, allowing many user customizations, such us selecting between different physics models, attaching particular processes to particles and, even modifying the source code to extract desired information not usually available to users.

The origin of the simulation's coordinate system (called MCZERO) is located approximately at the front face of Horn 1 and along the trajectory of the primary proton beamline. The position convention follows right-handed orientation Cartesian coordinates. The longitudinal axis ( $\mathrm{Z}$ axis) direction overlaps with the beamline (i. e. $58 \mathrm{mrad}$ downwards). The $\mathrm{Y}$ axis is vertical. Figure 2.8 shows the target in LE010 configuration (explained in Section 2.3) and Horn 1 positioned in the MC coordinate system.

The simulation starts with a $120 \mathrm{GeV}$ kinetic energy primary proton beam with a Gaussian beam transverse profile distribution $(\sigma=1.1 \mathrm{~mm})$. The main geometrical

\footnotetext{
${ }^{7}$ The GEANT4 version used is geant4.2.p03.
} 
details have been considered: the NuMI Target Hall including every component, the decay pipe, the absorbers, etc. However, the flux prediction is more sensitive to some elements than others. As described in Section 2.5, any mismodeling, particularly concerning the focusing process (the target and horns) can lead to a bad prediction of the flux. The simulation chain ends at the point where a neutrino is produced.

We use the GEANT4 hadronic model package called FTFP_BERT, that combines the FRITIOF precompound model [21] for processes with energies greater than $4 \mathrm{GeV}$ and the Bertini cascade model for energies less than $5 \mathrm{GeV}$ [22]. FTFP_BERT also incorporates the standard electromagnetic processes. More details about this model package and their agreement with measured data is discussed in Chapter 4.

The g4numi output is a ROOT-based n-tuple file with a record of all information of the neutrino production. Neutrino generators like GENIE [23], used in MINERvA as the standard MC generator, use the meson decay points and momenta to generate neutrinos and simulate their interactions in a detailed detector geometry.

G4numi also calculates the probability for a neutrino to be produced in a particular direction given its parent kinematics. "Forcing" this direction changes the neutrino energy as well (as in Equation 2.1). The probability of this occurring can be stored as a "weight" along with the new energy to make fast calculations of the flux in particular points in the beamline. The flux calculation at a single point is a good approximation for the flux at an extended detector if its location is far enough away that sees the neutrino production as a point source. For detectors close enough to the neutrino production place to see it as an extended source, the flux at a single point in the detector is a rough estimation of the true flux through the detector, suitable for systematic uncertainty studies and to have a quick look of the neutrinos passing through a detector.

In this thesis, the flux for MINERvA (shown in Chapter 5 to 7 ) is integrated over its fiducial volume (see Section 3.5). For the extension of our procedure (Section 4) 
to other detectors we take NOvA Near Detector as example and the flux is calculated at the center of its front face.

\section{Flux challenges}

There are two main challenges when calculating NuMI flux using a simulation:

- Hadron production. The MC flux relies on the underlying hadronic models and their predictions can have significant disagreements with data since QCD is not a completely calculable theory at the energy scales of interest. Constraining hadron production models used in the simulation by applying external data is a major topic of this thesis and it will be cover extensively in Chapter 4 .

- Focusing uncertainties. Any geometrical mismodeling can lead to a bad flux prediction, especially around the target and horns system where the flux is very sensitive to the hadron directions. The simulation has an idealized setup, where the implementation of the volumes needs to often be simplified. On the other hand, the parameters involved in NuMI's construction such as the location of beamline elements have inherently associated uncertainties. The MC simulation is used to calculate the effect of those uncertainties on the flux. This is covered in Section 2.5. 


\section{$2.3 \quad$ NuMI fluxes}

NuMI has been constructed as a tunable beamline. Different energy spectra and intensities of the neutrino flux can be achieved by changing the magnitude and direction of the magnetic field, as well as the relative distances between the target, Horn 1 and Horn 2. The idea is to be able to select and enhance the focusing of particles produced in a specific kinematic phase space and have a flexible beam energy. This is a key feature of NuMI that allows the exploration of neutrino oscillations at different $\Delta m^{2}$ (Section 1.2).

The NuMI main modes are called Low Energy (LE) and Medium Energy (ME). During the LE era, the horns positions remain fixed while the target was remotely movable, allowing experimenters to tune the neutrino energy. As was mentioned in Section 2.2.2, the target, Budal monitors and baffle were placed in the same carrier in order to make this change easily. For ME, the Horn 2 has been moved downstream from Horn1 while the target was moved upstream, producing the same energy shift in the flux spectrum but more efficiently than just moving the target.

The direction of the current pulsed to the horn conductors can be selected to focus or defocus hadrons with a particular charge. When $\pi^{+}$(and $K^{+}$) are focused, it enhances the muon neutrino component of the flux. This mode is called "Forward Horn Current" (FHC). The opposite current direction creates a magnetic field that focuses mainly $\pi^{-}$to enhance the muon antineutrino flux. This mode is called "Reverse Horn Current" (RHC).

\section{Low Energy Beams}

As was mentioned, in the LE run the target is longitudinally movable and the current value can be selected. The convention for LE configurations is "LEXXXzYYYi". The $\mathrm{z}=0$ position $(\mathrm{LE} 000 \mathrm{z})$ corresponds the place where the target is inserted into 
the Horn 1 as far as it can physically goes without electrical arcing between the target and Horn 1. XXX is the distance upstream from $\mathrm{z}=0$ (in $\mathrm{cm}$ ). The current is expressed as YYYi, where YYY is a reference magnitude (in kA), approximately the value used in the configuration. Table 2.3 contains the LE runs during the MINERvA run period.

\begin{tabular}{|c|c|c|c|}
\hline Name & Configuration & Current (kA) & Displacement from z=0 (cm) \\
\hline LE FHC & LE010z185i & 182.1 & -10.0 \\
LE RHC & LE010z-185i & -182.1 & -10.0 \\
\hline LE OHC ("horn off") & LE010z000i & 0 & -45.0 \\
Pseudo ME FHC & LE100z200i & 196.8 & -100.0 \\
Pseudo ME RHC & LE100z-200i & -196.8 & -100.0 \\
Pseudo HE FHC & LE250z200i & 196.8 & -250.0 \\
\hline
\end{tabular}

TABLE 2.3: Naming convention and parameters for LE NuMI runs for MINERvA data period.

The LE010z185i and LE010z-185i configurations were the long term runs for the LE era (2005-2012) and they have been used to achieve the physics goals of the MINERvA [9] and MINOS [24] experiments. The main result of this thesis is the flux determination for these configurations. The target is positioned $10 \mathrm{~cm}$ from $\mathrm{z}=0$ (as a visual aid, Figure 2.8a shows the target location respect to the Horn 1 in the MC coordinate system, i.e, LE010z).

The black line in Figure 2.12 shows the on-axis flux spectrum for $\nu_{\mu}$ in "LE010z185i" with its characteristic peak around $3 \mathrm{GeV}$ ("LE010z-185i" has similar peak for $\bar{\nu}_{\mu}$ ) and its long energy tail that extends up to $120 \mathrm{GeV}$.

By moving the target upstream with respect to "LE010z" the peak energy shifts to higher values (Table 2.3). The LE100z200i and LE100z-200i configurations have $100 \mathrm{~cm}$ target displacement from $\mathrm{z}=0$ and their peaks are at $5 \mathrm{GeV}$ for $\nu_{\mu}$ and $\bar{\nu}_{\mu}$, respectively. In the "LE250z200i" configuration the target is moved $250 \mathrm{~cm}$ 
from $\mathrm{z}=0$ and the peak shifts to $8 \mathrm{GeV}$. NuMI ran in these configurations for short periods of time allowing systematic cross-checks by measuring the neutrino interaction rates in MINOS and MINERvA in a given neutrino energy bin but with different hadrons contributing in the different tunes. The spectrum when turning the horn currents off is also shown and we can note that the flux decreases significantly in this configuration.

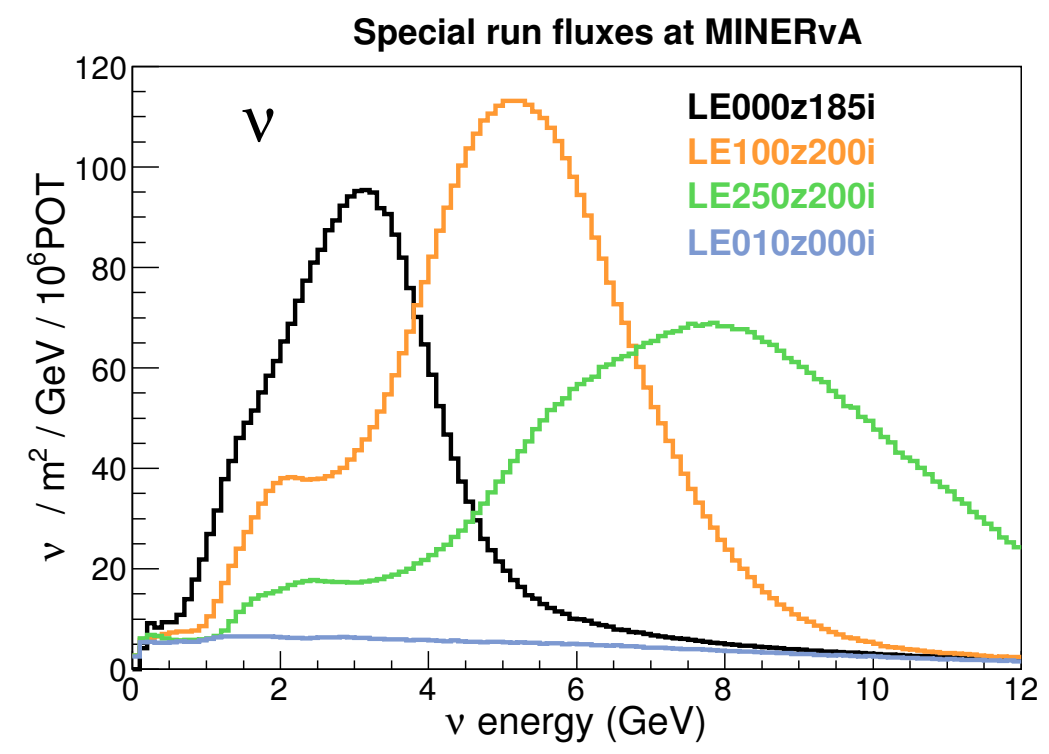

FIG. 2.12: MC $\nu_{\mu}$ fluxes for LE runs.

Figure 2.13 shows the predicted yields of $\pi^{+}$that leave the target to generate neutrinos that pass through MINERvA in different NuMI configurations. For LE010z185i, most of the $\pi^{+}$are focused in the region between 2-15 GeV/c longitudinal momentum and transverse momentum less than $600 \mathrm{MeV}$. The biggest contribution comes from $p_{Z}=7 \mathrm{GeV} / \mathrm{c}$ as we expect for a $3 \mathrm{GeV}$ LE peak using Equation 2.1. For LE010z000i, only pions with very small transverse momentum will decay, rather than hitting material, and produce neutrinos that are forward enough to pass through MINERvA. This is the reason why the particle focusing is 
important in any modern conventional neutrino beams.

The LE100z200i and LE250z200i configurations focus higher momenta $\pi^{+}$for two reasons:

First, when the target is moved away from Horn 1, highly energetic pions, which in LE pass neck-to-neck by the horns and decay to neutrinos unlikely to travel on-axis, can be focused by both horns now. This is the main contribution to the spectrum as can be seen in the "underfocused" plot in Figure A.1.

Second, some "unfocused" and "horn2-only" pions are now part of the "horn1only", "underfocused" and "overfocused" categories. For example, a fast pion with small transverse momentum that is focused just by Horn 2 in LE can pass the Horn 1 with the same transverse momentum in the special runs. See Figures A.2 to A.7.

\section{Medium Energy Beam}

By moving the target upstream, the peak energy of the on-axis neutrino flux increases. But this procedure is more efficient when the distance between horns is simultaneously enlarged. The reasoning is the same as in the "special runs": more highly energetic neck-to-neck pions can be caught by the horns before their transverse momentum make them diverge from the beamline and crash into the walls. However, moving Horn 2 further away is more efficient than just moving the target. In this way, the ME flux spectrum is approximately $30 \%$ greater than in LE100z200i configuration.

The bottom left plot in Figure 2.13 shows the $\pi^{+}$yields off of the target. A significant shift to larger longitudinal momentum values can be noted in comparison to LE010. The result is the flux spectrum in Figure 2.14 (red line). The peak is approximately at $6 \mathrm{GeV}$ with a small bump at $3 \mathrm{GeV}$ due to overfocused pions.

The NOvA Near Detector is located $12.26 \mathrm{mrad}$ off the beam axis and, due to Equation 2.1, the neutrino energy spectrum is peaked at lower energy, and is 

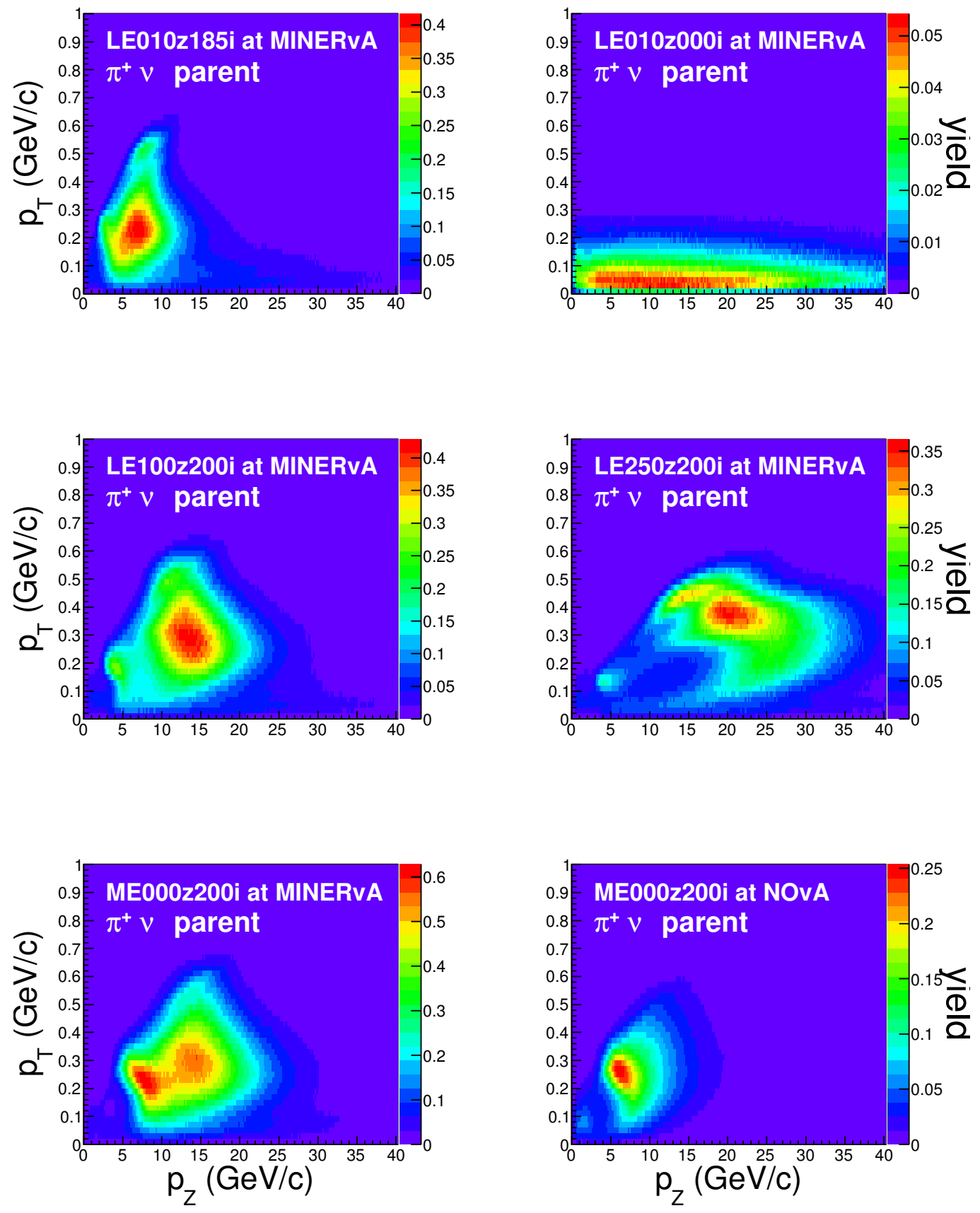

FIG. 2.13: $\pi^{+}$yields that exit the target for different beam configurations at MINERvA and NOvA Near Detector. 
significantly narrower than the spectrum one sees on axis. See Figure 2.14.

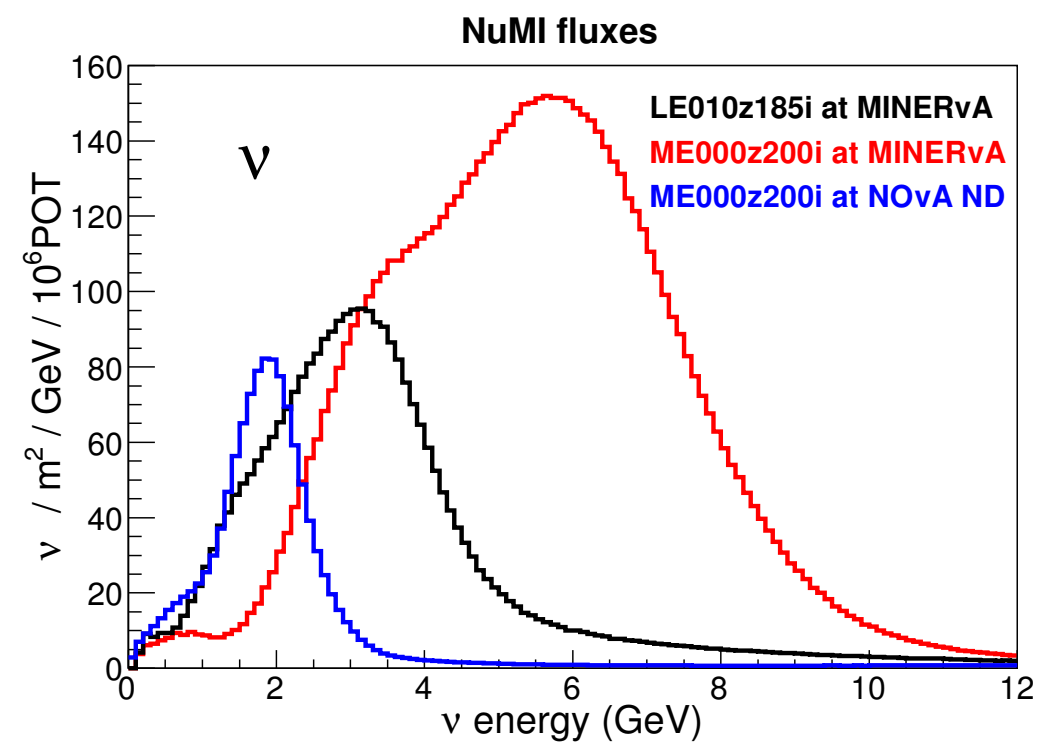

FIG. 2.14: $\mathrm{MC} \nu_{\mu}$ fluxes for ME runs in the MINERvA and NOvA Near Detectors. LE010185i is shown as a reference.

\subsubsection{Beam components}

Figure 2.15 shows the flux components for different beam configurations. The neutrino type focused in FHC modes are muon neutrinos with a small (few percentage) background contamination from muon antineutrinos, that is completely unfocused. The background comes mainly from mesons produced outside of the target and some "horn2-only" mesons. The electron neutrino flux in LE010z185i represents less than $1 \%$ of the total flux and about $0.8 \%$ in the focusing peak according to the simulation.

Conversely, muon antineutrinos focused in LE010z-185i flux are the main component up to $8 \mathrm{GeV}$. For higher energies $(>10 \mathrm{GeV}$ ), when the flux comes mostly from unfocused meson parents, the muon neutrino contribution is dominant because 
more positive mesons are produced than negative mesons in the target, but $K^{+}$are also focused. See Figure 2.15. 

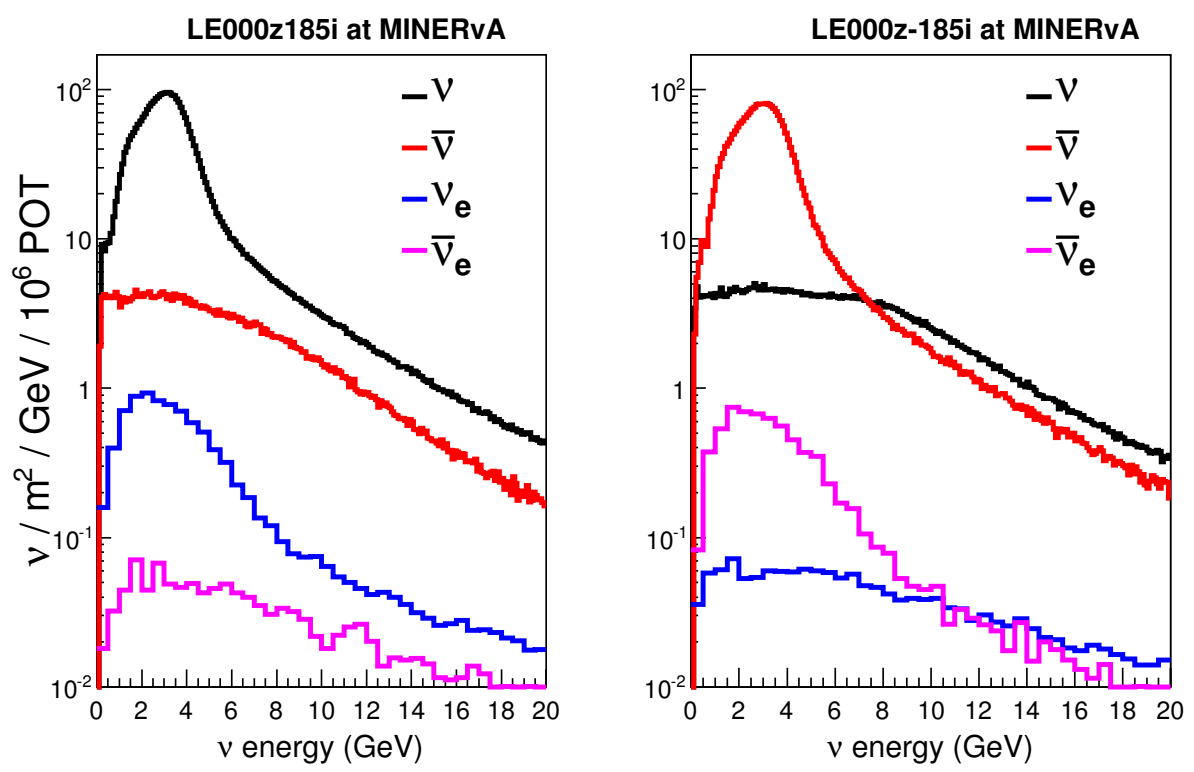

(a) LE010z185i and LE010z-185i beam configuration.
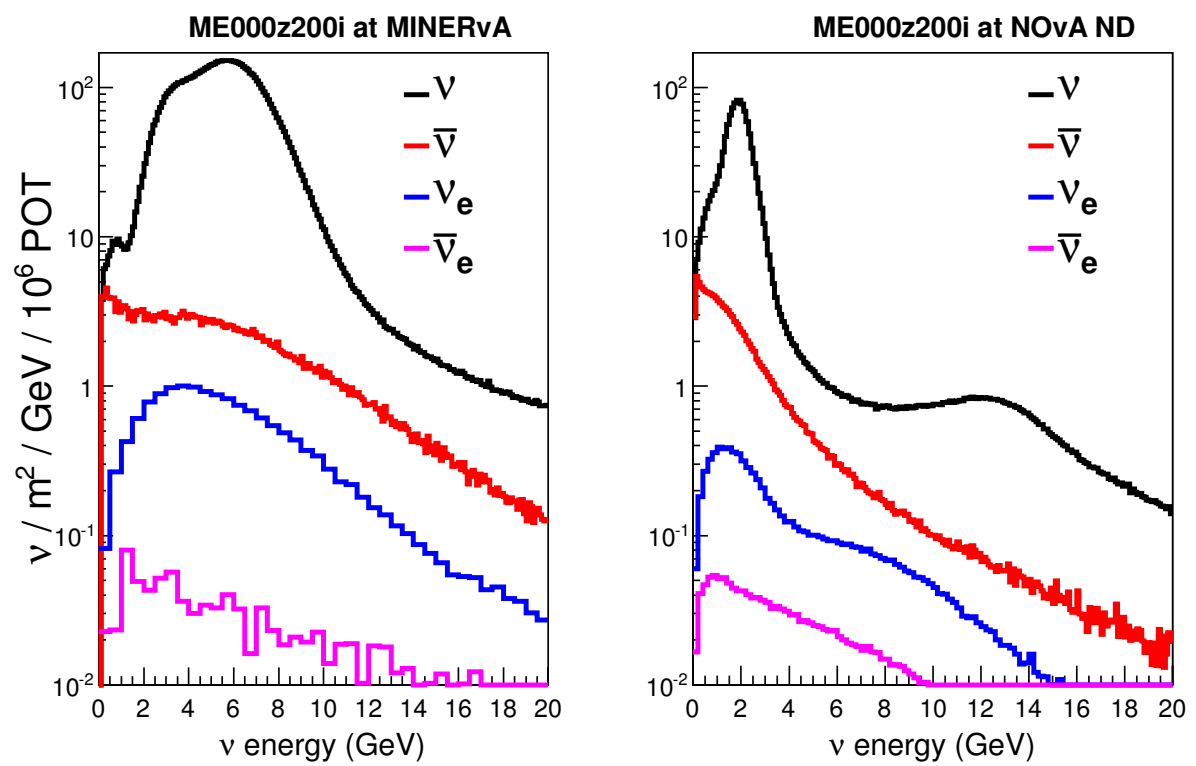

(b) ME000z200i beam configuration.

FIG. 2.15: Neutrino components for LE and ME beams. 


\subsection{Hadronic cascade in NuMI}

When the primary proton beam interacts with the target (carbon) or with any other NuMI volume (made of carbon, aluminum, iron, helium, etc) it generates a hadronic cascade creating pion, kaons, protons and other particles. Some of them decay to make neutrinos, but others may interact in any NuMI component. The hadronic interaction chain (i.e., the history of how a neutrino has been produced) is very important because each one of these interactions relies on a MC model and we need to know how well that model is representing nature.

The NuMI hadronic cascade is composed of many interactions of different particles on different materials. The objective of this section is to recognize which of these interactions are relevant for the neutrino flux in order to look for data to apply in the correction procedure described in Chapter 4. Most of the analysis is based primarily on $\nu_{\mu}$ in LE010185i (with some $\nu_{e}$ plots). The corresponding plots for NOvA have been placed in appendix A.

\subsubsection{Neutrino ancestries}

Figures 2.16a and $\mathrm{b}$ show the LE010z185i flux split in terms of the parent and grandparent identity for the main particle contributions that passes trough MINERvA. NOvA plots are in Figures A.9 and A.10. The parent identity plots are extended up $40 \mathrm{GeV}$ but for simplicity, the corresponding grandparent plots are only shown to $10 \mathrm{GeV}$.

The main contribution for muon neutrinos at energies less than $20 \mathrm{GeV}$ comes from $\pi^{+}(\sim 97 \%)$. In terms of the grandparents, they come from the primary proton $(\sim 57 \%)$, secondary and tertiary protons $(\sim 15.8 \%)$ and $\pi^{+}(\sim 10 \%)$. The high energy tail ( $\nu$ energy $>20 \mathrm{GeV}$ ) is dominated by $K^{+}$.

Electron neutrinos up to $10 \mathrm{GeV}$ come, basically, from $\mu^{+}$decays $(\sim 85 \%)$ and 
$K^{+}(\sim 11 \%)$, and most of their grandparents are $\pi^{+}(\sim 84 \%)$. For higher energies, electron neutrino generation is dominated by $K^{+}$with a small contribution from neutral kaons.

For NOvA, the trend is similar but the transition between parent identity is $6 \mathrm{GeV}$ for $\nu_{\mu}$ and $5 \mathrm{GeV}$ for $\nu_{e}$.

\subsubsection{Interaction map}

A tool has been developed to make a complete survey of all interactions which happened in NuMI that lead to a neutrino. This was done to obtain a more quantitative view of which projectile and produced particles, as well which materials, are relevant to understanding the flux. This "interaction map" calculates the average number of hadronic interactions per each neutrino type.

Figures 2.17 and 2.18 are examples of two interaction maps of $\nu_{\mu}$ in LE010z185i integrated over 0-20 GeV. The corresponding NOvA maps are in Figures A.11 and A.12. Fields with less than 0.001 interactions per neutrino are not filled. The horizontal axes have the relevant materials.

The vertical axis of Figure 2.17 has all possible hadron projectiles. The main contributions come from interactions that happen on carbon, basically in the target. Other relevant materials are aluminum (horn inner conductors), iron (decay pipe walls), and air (target hall). Other significant projectiles are $\pi^{+}$and neutrons. Due to the neutrino energy cut at $20 \mathrm{GeV}$ for these plots, the $K^{+}$contribution does not appear.

Figure 2.18 shows the interaction map for incident protons. This map contains the number of produced particles per $\nu_{\mu}$ vs. the NuMI target materials (numbers less than 0.001 are not shown). Interactions with incident protons happen mainly in carbon, producing protons and $\pi^{+}$. 

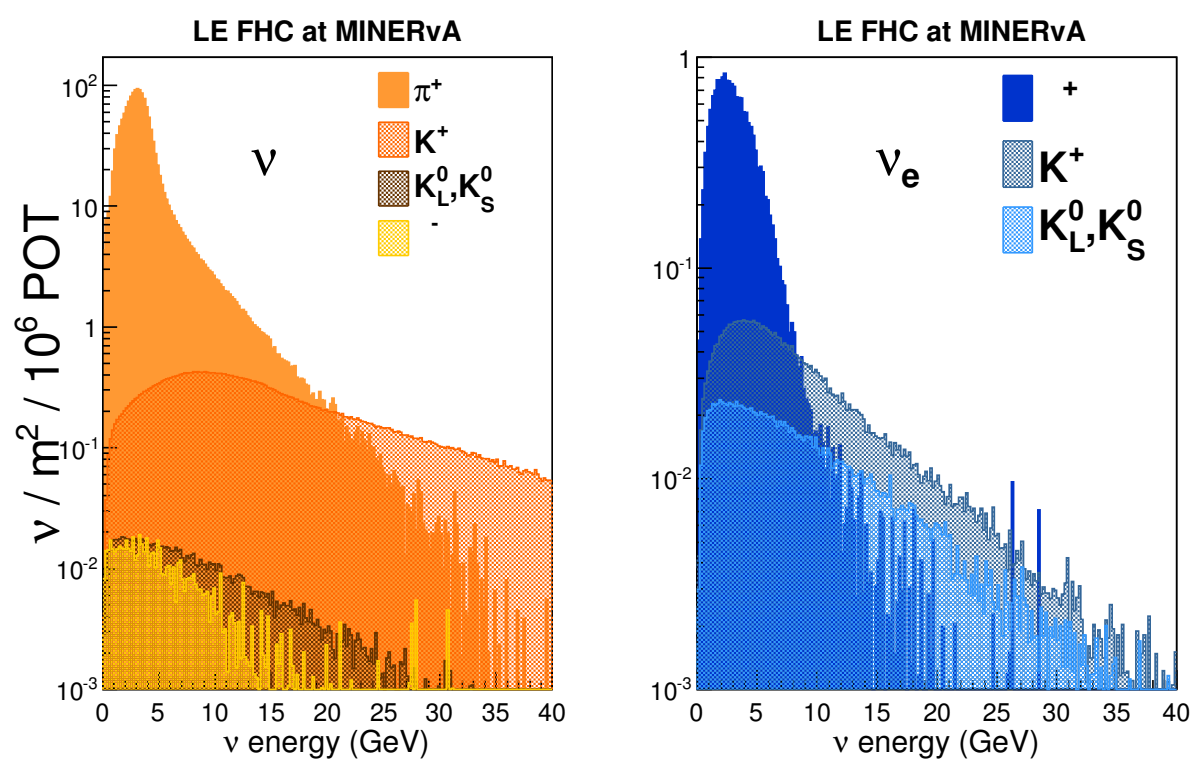

(a) Parents.
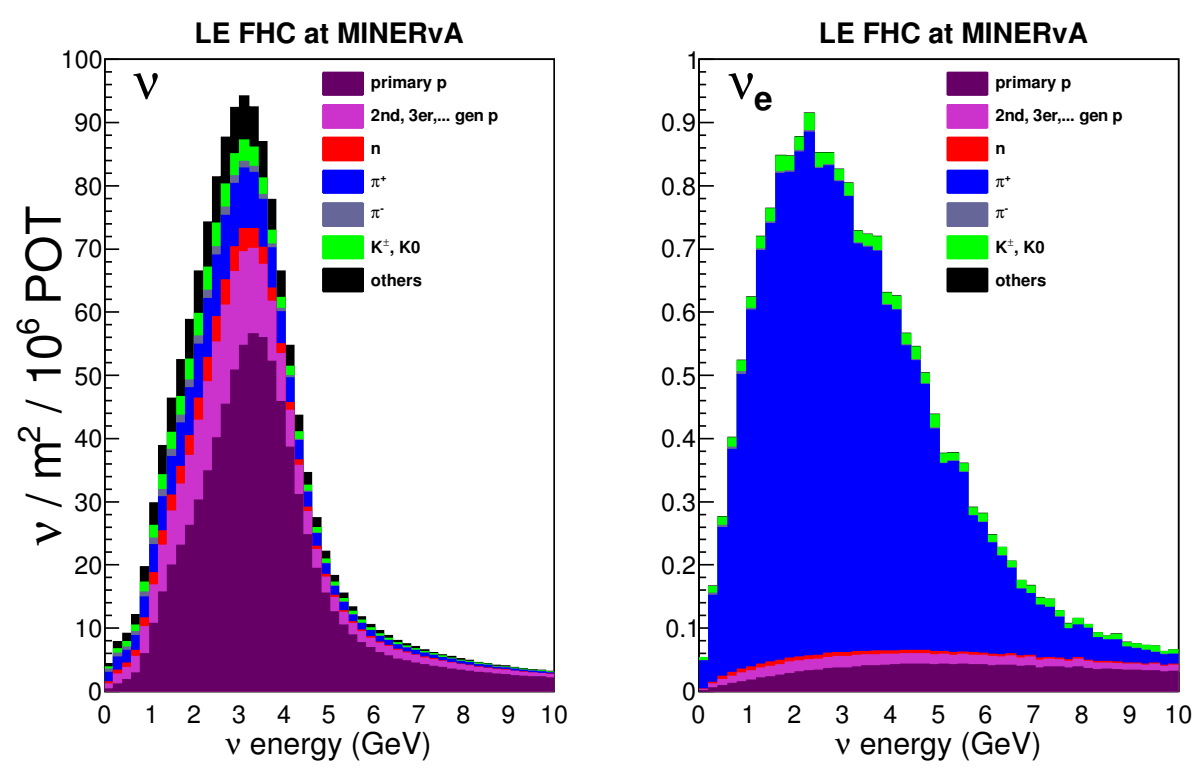

(b) Grandparents.

FIG. 2.16: $\nu_{\mu}$ and $\nu_{e}$ flux in the LE010z185i configuration at MINERvA split in their ancestry. 


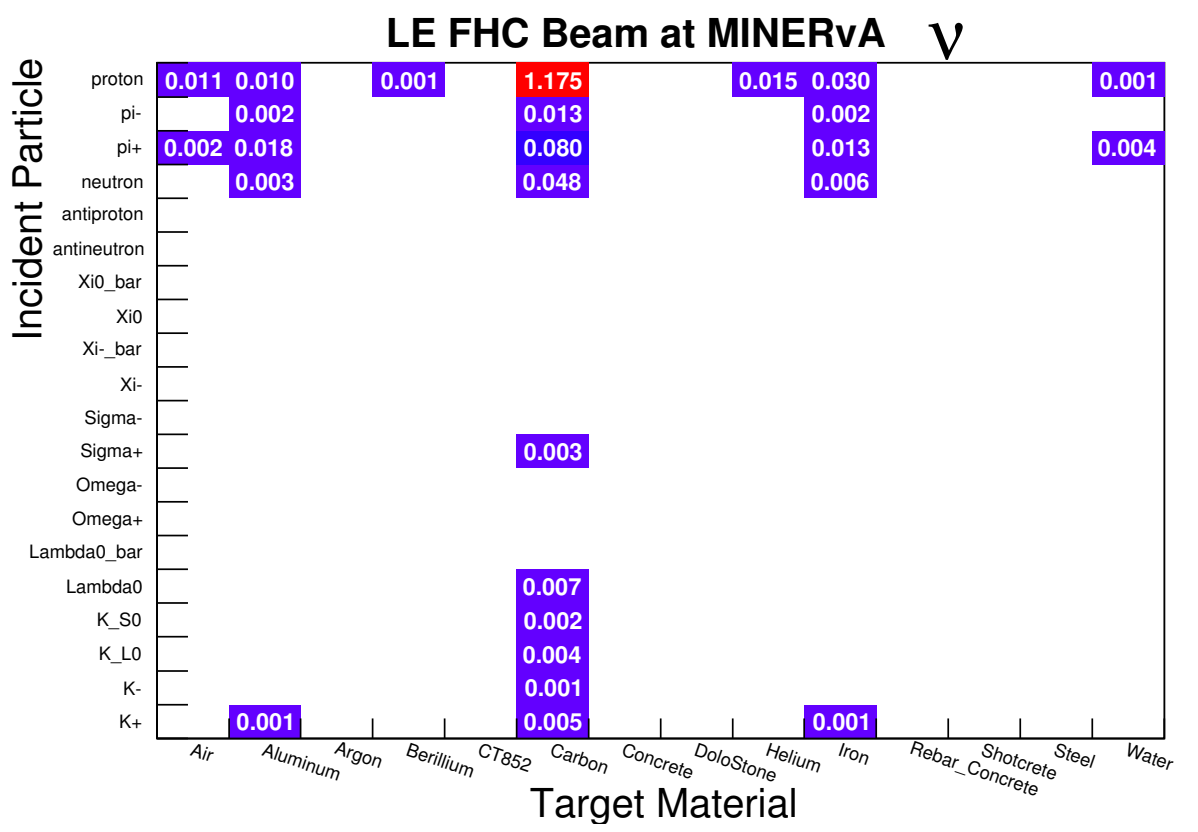

FIG. 2.17: Interaction map of incident particles vs. target material for $\nu_{\mu}$ flux passing through MINERvA in the LE010z185i configuration (numbers less than 0.001 are not shown). 


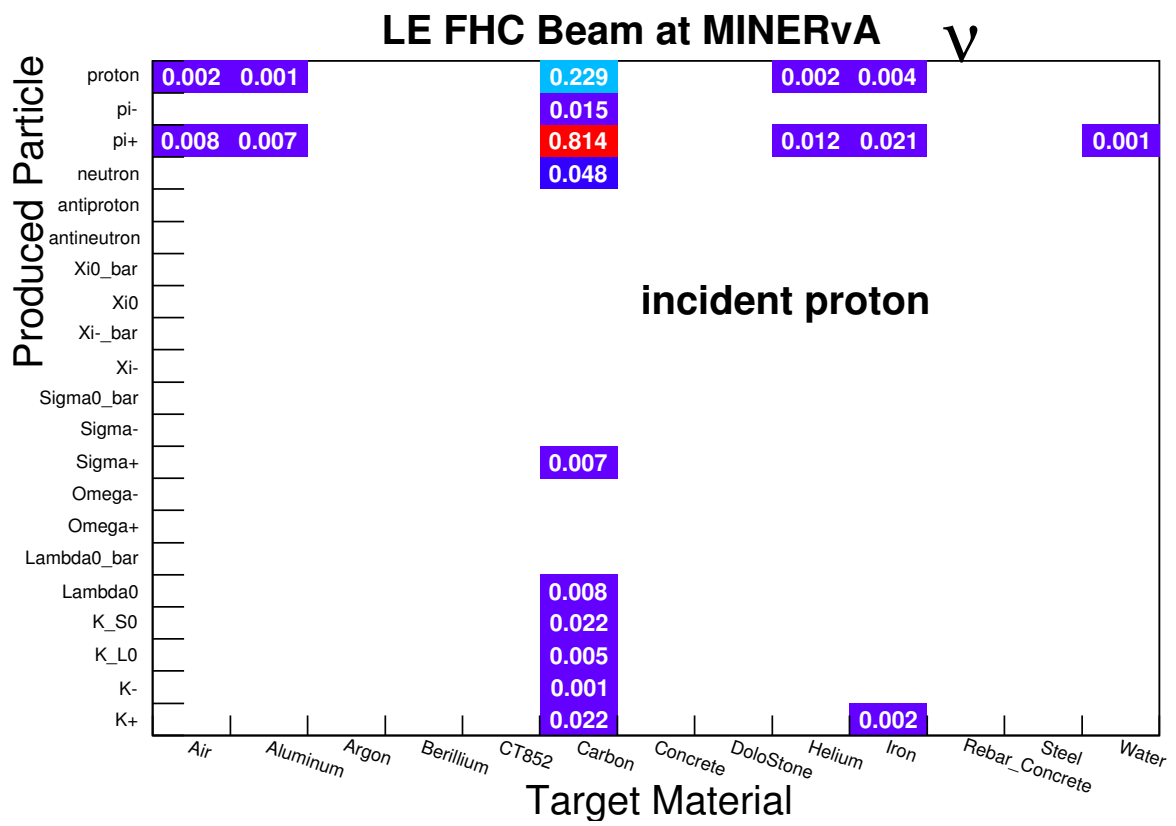

FIG. 2.18: Interaction map for proton projectiles. The map shows the number of produced particles per $\nu_{\mu}$ event vs. target material at MINERvA in LE010z185i configuration (numbers less than 0.001 are not shown). 


\subsubsection{Hadronic production}

The kinematic distribution of hadrons produced in primary proton interactions on the target is shown in Figure 2.19 for 6 particles: $\pi^{+}, \pi^{-}, K^{+}, K^{-}$, protons and neutrons in terms of Feynman-x and transverse momentum. This corresponds to muon neutrinos in LE010z185i and similar plots for muon neutrino in NOvA are in Figure A.13. For charged pions and kaons, an additional line on the top has been placed with the approximate expected neutrino energy using Equation 2.1. The plots are normalized to $10^{6}$ POT's and the bin sizes are: $\Delta x_{F}=0.02$ and $\Delta p_{T}=0.02 \mathrm{GeV} / \mathrm{c}$.

The region of interest for $\pi^{+}$is $x_{F}<0.15$ and $0.05<p_{T}<0.55$. This region contributes to the focusing peak. $\pi^{-}$and $K^{-}$look defocused in the same region and their contribution is very small. $K^{+}$production has an extensive area, but the region of interest is $x_{F}>0.17$ where they become dominant in the neutrino spectrum. Protons have a wider $x_{F}$ range and it can be split in two regions: one for $x_{F}<0.95$ that has a peak around 0.5 and other for $x_{F}>0.95$ that corresponds to protons likely to be quasi-elastics (see Chapter 4). Neutrons make a very small contribution to the LE flux.

\subsubsection{Beam attenuation}

The relevant amount of material traversed by hadrons in the neutrino chain leading to a muon neutrino in LE010z185i is shown in Figure 2.20. Similar plot for NOvA can be found in Figure A.14. The importance of this quantity in correcting the flux is seen in Section 4.2.1. The figure corresponds to the primary and secondary protons passing through the target $(\mathrm{C})$, and $\pi^{+}$crossing the target $(\mathrm{C})$, the horn inner conductors $(\mathrm{Al})$, the decay pipe volume $(\mathrm{He})$ and the decay pipe walls $(\mathrm{Fe})$. The horizontal axis is the material traversed $\left(\mathrm{mol} / \mathrm{cm}^{2}\right.$ units) and the bin size is 

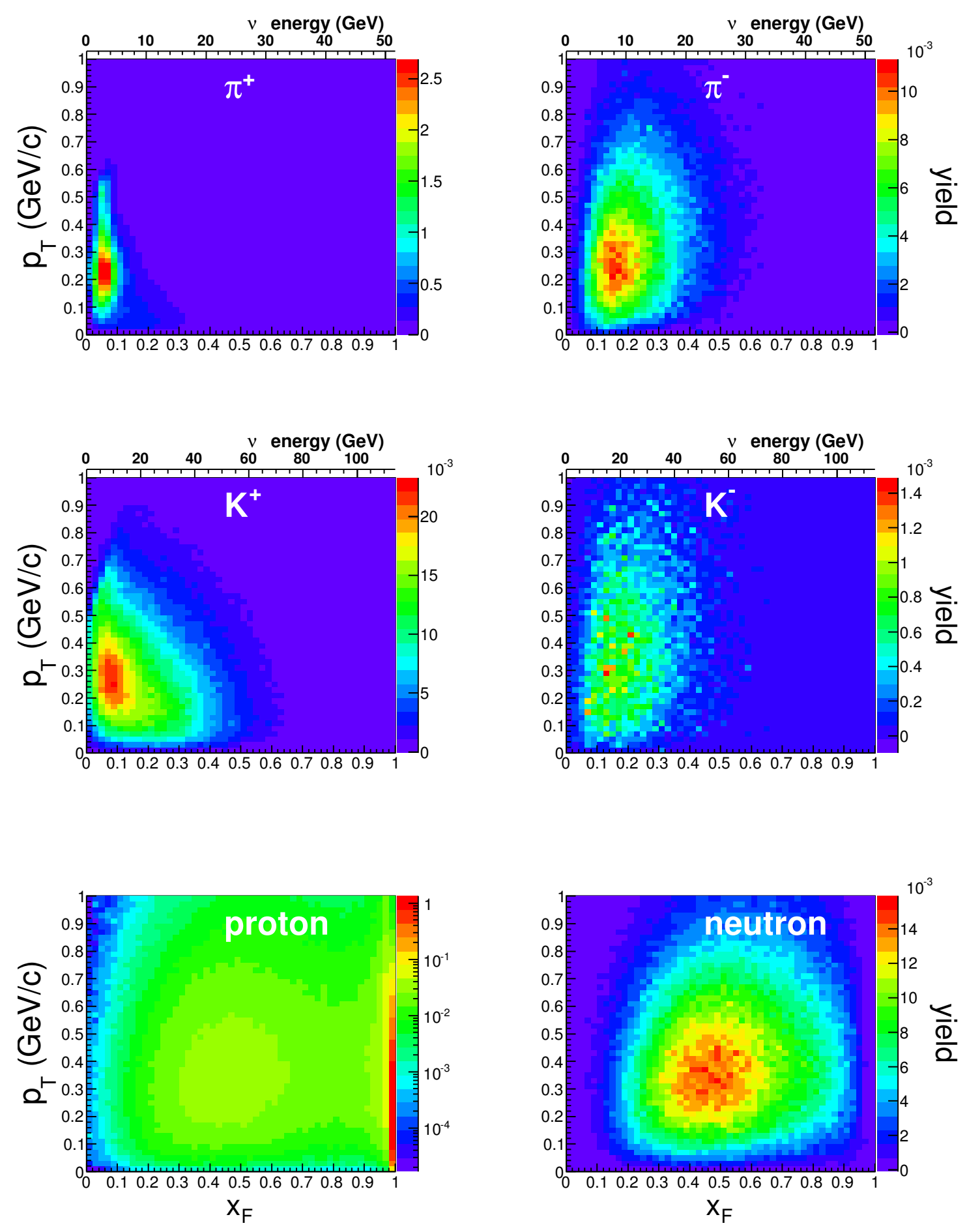

FIG. 2.19: Hadron production from the primary proton beam interacting in the target for $\nu_{\mu}$ flux passing through MINERvA in the LE010z185i configuration. 
$0.1 \mathrm{~mol} / \mathrm{cm}^{2}$.

The top left plot corresponds to the primary proton in the target and shows the characteristic exponential behavior one expects. The peak at the $14 \mathrm{~mol} / \mathrm{cm}^{2}$ come from protons that leave the target without interacting. The top right plot shows the secondary protons per momentum in log scale with a peak at high momentum due to quasi-elastic scattering.

The rest of the plots are for $\pi^{+}$neutrino parents in different materials. In the target, most of them traverse less than $2.5 \mathrm{~mol} / \mathrm{cm}^{2} \sim 20 \mathrm{~cm}$. Though the horns inner conductor is thin $(2-4 \mathrm{~mm})$, the horns are long and skinny resulting in rather long path lengths (around $1 \mathrm{~mol} / \mathrm{cm}^{2} \sim 10 \mathrm{~cm}$ ). In the decay pipe it is $1 \mathrm{~mol} / \mathrm{cm}^{2} \sim 275 \mathrm{~m}$ but with a limit at $2.45 \mathrm{~mol} / \mathrm{cm}^{2} \sim 675 \mathrm{~m}$, the length of the pipe. The decay pipe walls are negligible.

Table 2.4 summarizes the density and mass number of the NuMI volumes included in Figure 2.20. This corresponds to the volumes where the attenuation correction is applied (see Chapter 4).

\begin{tabular}{|l|c|c|c|}
\hline Volume & Material & Mass number $(\mathrm{g} / \mathrm{mol})$ & Density $\left(\mathrm{g} / \mathrm{cm}^{3}\right)$ \\
\hline TGT and BM & carbon & 12.01 & 1.78 \\
IC & aluminum & 26.98 & 2.7 \\
DVOL & Helium & 4.003 & 0.000145 \\
DPIP & iron & 55.85 & 7.87 \\
\hline
\end{tabular}

TABLE 2.4: Material information for volumes considered in Figure 2.20.

\subsection{Focusing uncertainties}

In this section I present the uncertainties in the flux that come from the focusing system. Some of these efforts were inherited from MINOS studies [25] like 

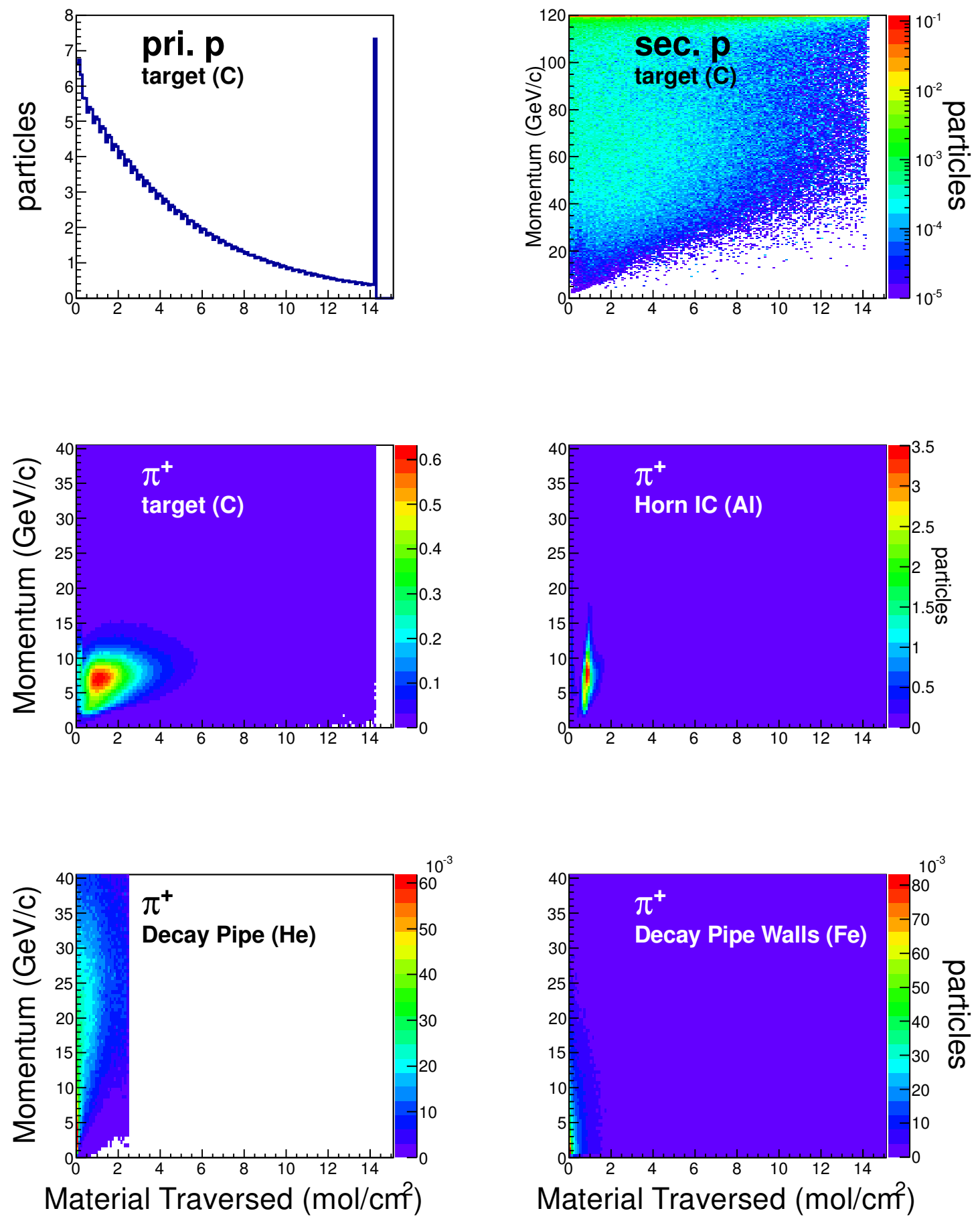

FIG. 2.20: Material traversed by particles in the NuMI beamline for $\nu_{\mu}$ flux passing through MINERvA in the LE010z185i configuration. 
the transverse horn offset, the baffle scraping, the POT counting and the horn current magnitude. We revisited the horn current distribution and added the horn inner conductor shape, target position and water layer around the inner conductor uncertainties.

We expect that those uncertainties directly related to the relation of target and horns as well as to the horn parameters impact the region just outside the focusing peak and have low impact for high energies. The reason for this is that all parameters involved (distances, materials, etc) have been optimized to have a small uncertainty on the flux peak (to be more precise, in the Far over Near ratio for oscillation studies) and there was a large effort to make the simulation very close to the actual setup. The biggest uncertainties are in the falling edge of the flux spectrum (4-6 GeV) as can be seen in the Figure 2.21 that shows the fractional shifts by 1 sigma in the LE FHC mode.

A brief description of each uncertainty is presented in the rest of the Chapter in the context of the $\nu_{\mu}$ flux in LE010z185i at MINERvA and MINOS. These were calculated using MC simulation considering the uncertainty on each parameter given by the NuMI beamline group. The general procedure is to vary each parameter by $\pm 1,2,3 \sigma$ around its accepted value and see the effect on the neutrino flux. Understanding an uncertainty means knowing, as well as we can, the effect of a change in that parameter. In our case, we need to see the effect on the flux when we vary a parameter. Some of the effects are clearly linear (like POT counting), while other effects need further investigation to see the exact functional dependency of the change. In this case, several sigma flux deviations were simulated and a second degree polynomial was used to find this form in different neutrino energy bins. The fractional shift on the flux is taken in each bin by evaluating the resulting polynomial at $\pm 1 \sigma$. 


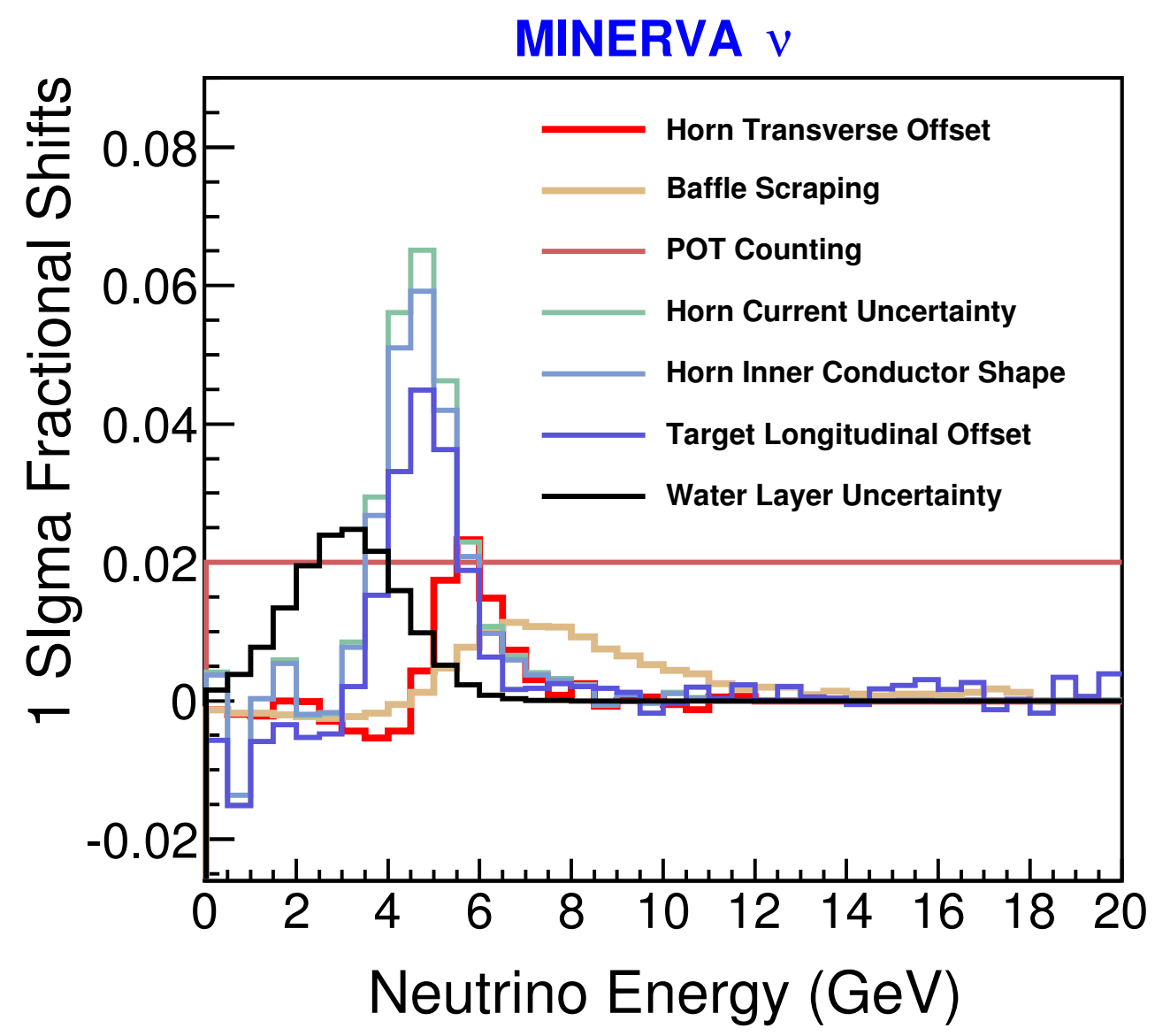

FIG. 2.21: Focusing fractional shifts for $\nu_{\mu}$ in the LE010z185i configuration at MINERvA. 


\subsubsection{Transverse horn offset}

This uncertainty was considered to be a $\sigma=1.0 \mathrm{~mm}$ offset. In Ref. [25] Horn 1 and Horn 2 misalignments were studied separately and the total effect is in the red line in Figure 2.21. That line also include smaller effects such as angular misalignments of the horns.

The result has the undesirable effect of reducing the flux in the focusing peak by $0.05 \%$ even while it increases by $2 \%$ the flux in the $4.5-8 \mathrm{GeV}$ energy range. For example, in the focusing peak, "horn2-only" mesons with small angles that need slight focusing by Horn 2, are now deviated in Horn 1 and become unlikely to contribute to the flux when we move the Horn 2 (in analogy can be made for "horn2-only" mesons). But moving the horns makes high energy mesons that would pass neck-to-neck be focused and become more likely to contribute to the on-axis flux.

\subsubsection{Baffle scraping}

This uncertainty comes from neutrino ancestors that are produced when the primary proton beam interacts with the baffle upstream of the target. It is estimated in two ways. First, by fitting the proton beam profile to a Gaussian and calculating the fraction of the beam expected to hit the baffle. Second, by measuring the beam-induced temperature change in the baffle and relating that to the number of interactions. This gives a fraction of $0.25 \%$ of the protons that hit the baffle [25].

Baffle scraping decreases the number of low energy pions, including those that contribute to the focusing peak. On the other hand, more high energy pions are able to be focused now. The orange line in Figure 2.21 shows a very small reduction in 0-5 GeV neutrino energy and a flux increase of no more $1 \%$ in the $5-11 \mathrm{GeV}$ region. 


\subsubsection{POT counting}

NuMI directly measures the number of protons in the beam using two toroid intensity monitor integrators [13] with an uncertainty of 1\% [25]. To take into account other beam-related parameters like its position and width, the uncertainty is conservatively taken as $2 \%$. The POT value is an overall normalization.

\subsubsection{Horn current}

The uncertainty on the horn current magnitude is estimated to be $1 \%$, generating a corresponding uncertainty in the magnetic field value. The falling edge of the flux peak increases by $6.5 \%$ when the current increases (as seen in the green line in Figure 2.21) and it decreases when the current decreases.

The skin depth effect $(\delta)$ of the conductors was reassessed [26] and it was found that the current is approximately uniformly distributed inside the conductor for $\delta \sim 7.7 \mathrm{~mm}^{8}$. We varied $\delta$ within its nominal uncertainty (due to the uncertainty on the magnetic permeability) and found no change in the flux, so the skin depth systematic was removed.

\subsubsection{Horn inner conductor shape}

The NuMI simulation was upgraded to incorporate a more accurate geometric and magnetic model of Horn 1 [27].

The main addition is a better representation of the inner conductor shape. Due to some limitations in the GEANT4 geometry package, the parabolic part was approximated by segmenting it into in small cones with $50 \mu \mathrm{m}$ length each one (this is called the "old horn model" in this thesis). Ideally the horn model would have an infinite number of infinitesimal length segments. The impact of improving

\footnotetext{
${ }^{8}$ This value correspond to $2.3 \mathrm{~ms}$ pulse duration and inner conductor thickness between $2-4 \mathrm{~mm}$
} 
the segmentation by making it 50 times finer ("new horn model"), and thus better modeled, is to reduce the flux up to $6 \%$ in the $4-6 \mathrm{GeV}$ range for LE FHC and up to $14 \%$ in $7-14 \mathrm{GeV}$ for ME FHC as can be seen in Figure 2.22. This suggests that this change especially affects mesons leaving the target and passing the neck in their trajectory before decay.

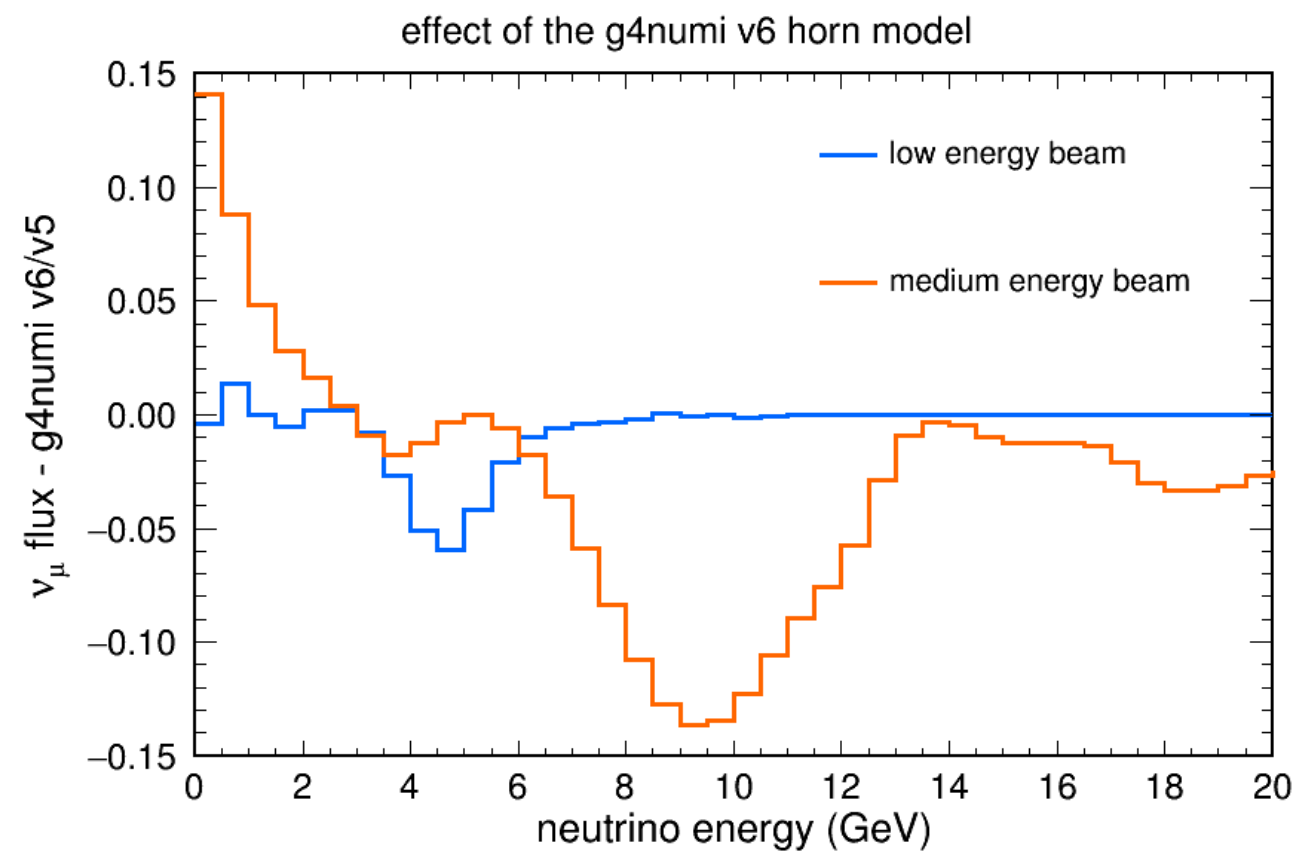

FIG. 2.22: Effect of the new horn model on the $\nu_{\mu}$ flux at MINERvA for the LE010z185i and ME000z200i configurations.

As is described in Section 3.5, the new horn model was not implemented for the current MINERvA published papers.

The new horn model has an effect that is very similar to the effect we see when we reduce the horn current by $0.8 \%$. Using this fact (and due to the time scale of this thesis), we reused the horn current magnitude uncertainty shape for this systematic assigning 100\% uncertainty. The result is the light blue line in Figure 


\subsubsection{Longitudinal target offset}

An optical survey has been made to determine the exact longitudinal position of the target after moving the target carrier between different NuMI configuration runs [28]. The uncertainties of these values are around $1 \mathrm{~mm}$. Any shift from the nominal positions shown in Table 2.3 affects the neutrino spectrum since the relative position target - Horn 1 is crucial in the particle focusing.

The precise position of the target was implemented in g4numi by splitting each NuMI beam configuration in run periods using the values given by the survey for the time when MINERvA starts taking data. Every run period is known as a playlist and it is named using the label minervaX, where $\mathrm{X}$ corresponds to a run period. This involves generating flux predictions for each playlist as can be seen in Table 2.5 .

Figure 2.23 shows the impact of the longitudinal target displacement in the $\nu_{\mu}$ flux for LE FHC mode. Moving the target upstream produces a decrease in the flux in the $4-6 \mathrm{GeV}$ energy region.

We use the longitudinal target position uncertainty from MINOS studies, that assume $1 \mathrm{~cm}$ uncertainty (blue line in 2.21) even when we use the survey data in our simulation. This value is clearly an overestimated value and needs to be revised in the future.

\subsubsection{Water layer uncertainty}

The horns are constantly sprayed with cooling water which is deposited around the inner conductor creating a thin layer. The sketch in Figure 2.24 shows the horn inner conductor from the transverse view (left side) and the longitudinal view (right 


\begin{tabular}{|c|c|c|}
\hline Beam configuration & Playlist & $\Delta Z(\mathrm{~cm})$ \\
\hline LE010z185i & minerva1 & +0.50 \\
& minerva7 & +0.82 \\
& minerva9 & -0.40 \\
& minerva13 & +0.87 \\
\hline LE010z-185i & minerva & +0.50 \\
& minerva5 & +1.15 \\
& minerva10 & +0.82 \\
\hline LE010z000i & minerva6 & +0.82 \\
\hline LE100z200i & minerva2 & +0.43 \\
& minerva11 & +0.83 \\
\hline LE100z-200i & minerva3 & +0.43 \\
& minerva12 & +0.83 \\
\hline LE250iz200i & minerva4 & +0.43 \\
& minerva8 & -0.09 \\
\hline
\end{tabular}

TABLE 2.5: MINERvA LE flux playlists. The shift in the target position $\Delta z$ is with respect to the nominal position. 


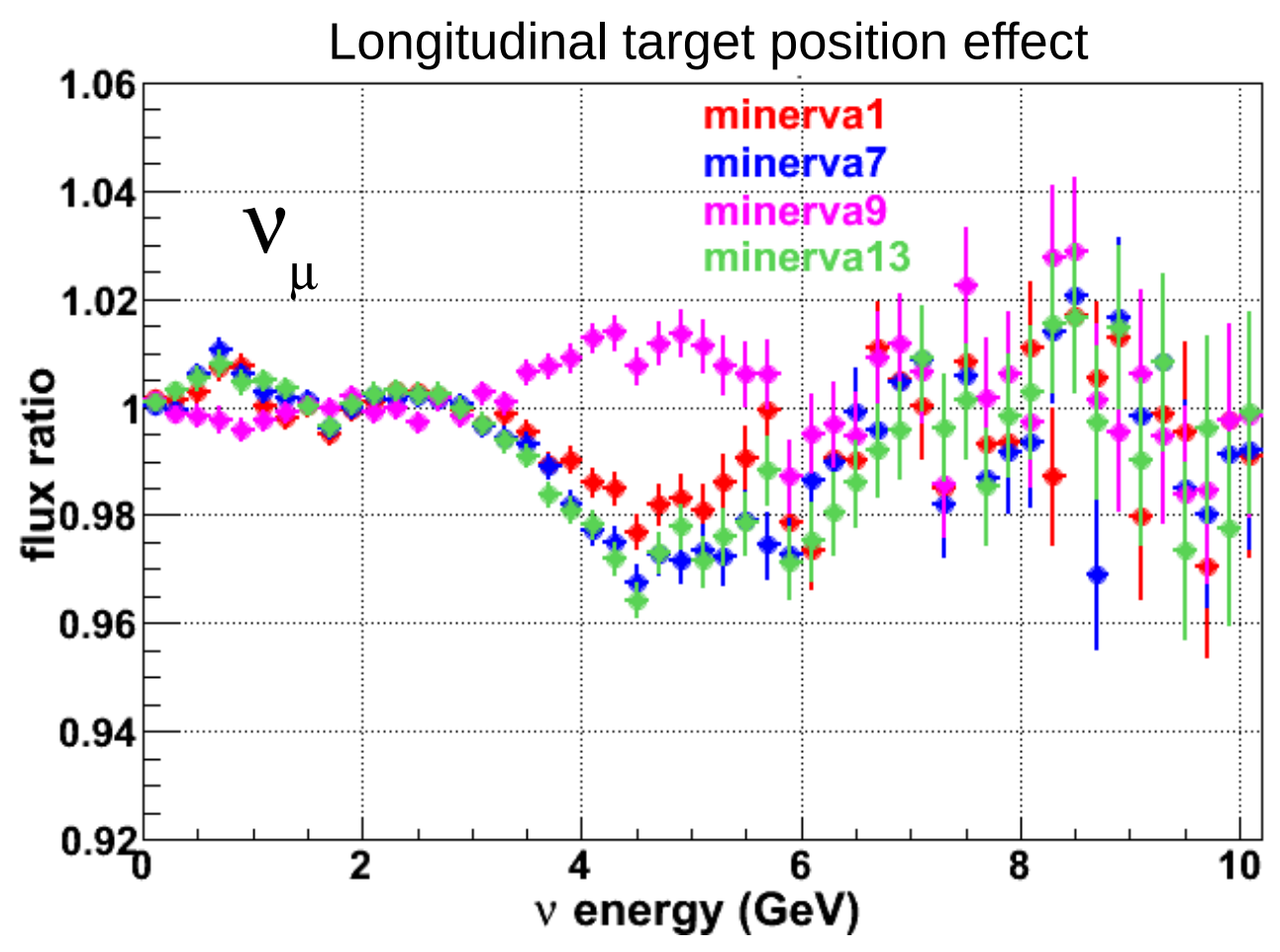

FIG. 2.23: Longitudinal target position displacement effect in the $\nu_{\mu}$ flux at MINERvA. 
side) located between $a<r<b$ (light violet curve).
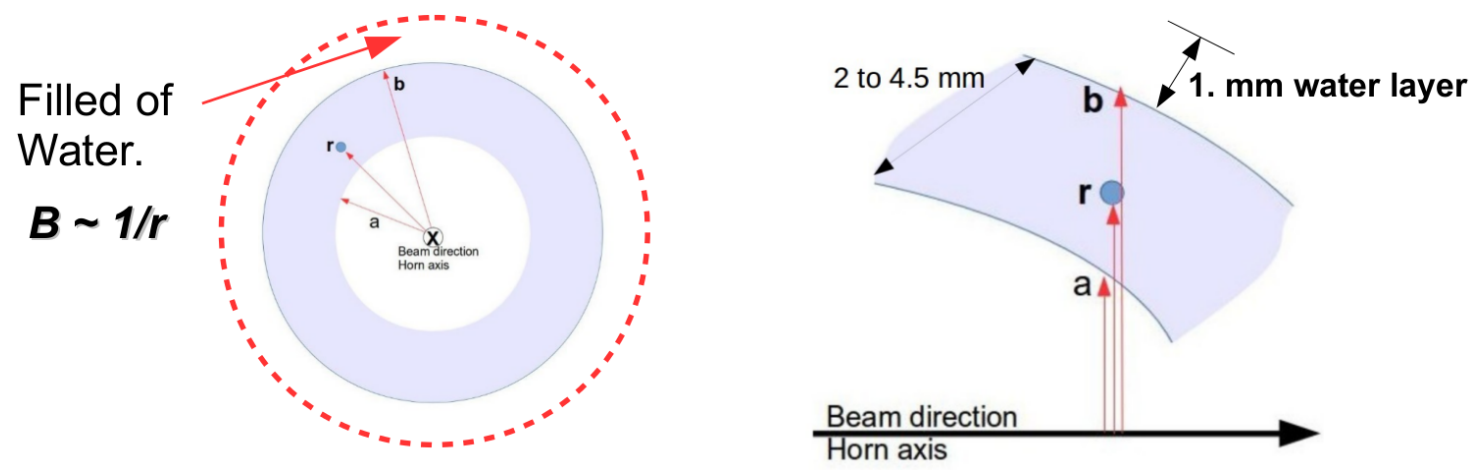

FIG. 2.24: Sketch of the position of the water layer around the horn inner conductor.

A $1 \mathrm{~mm}$ thick water layer was implemented in g4numi [29] (the NuMI Beam group estimates $1 \mathrm{~mm}$ thickness as the maximum expected) and the effect on the $\nu_{\mu}$ flux in LE010z185i is shown in Figure 2.25: a four percent reduction on the flux to due the meson absorption on the water for energies less than $4 \mathrm{GeV}$. The uncertainty applied was $0.5 \mathrm{~mm}$ (blue line in Figure 2.21). 


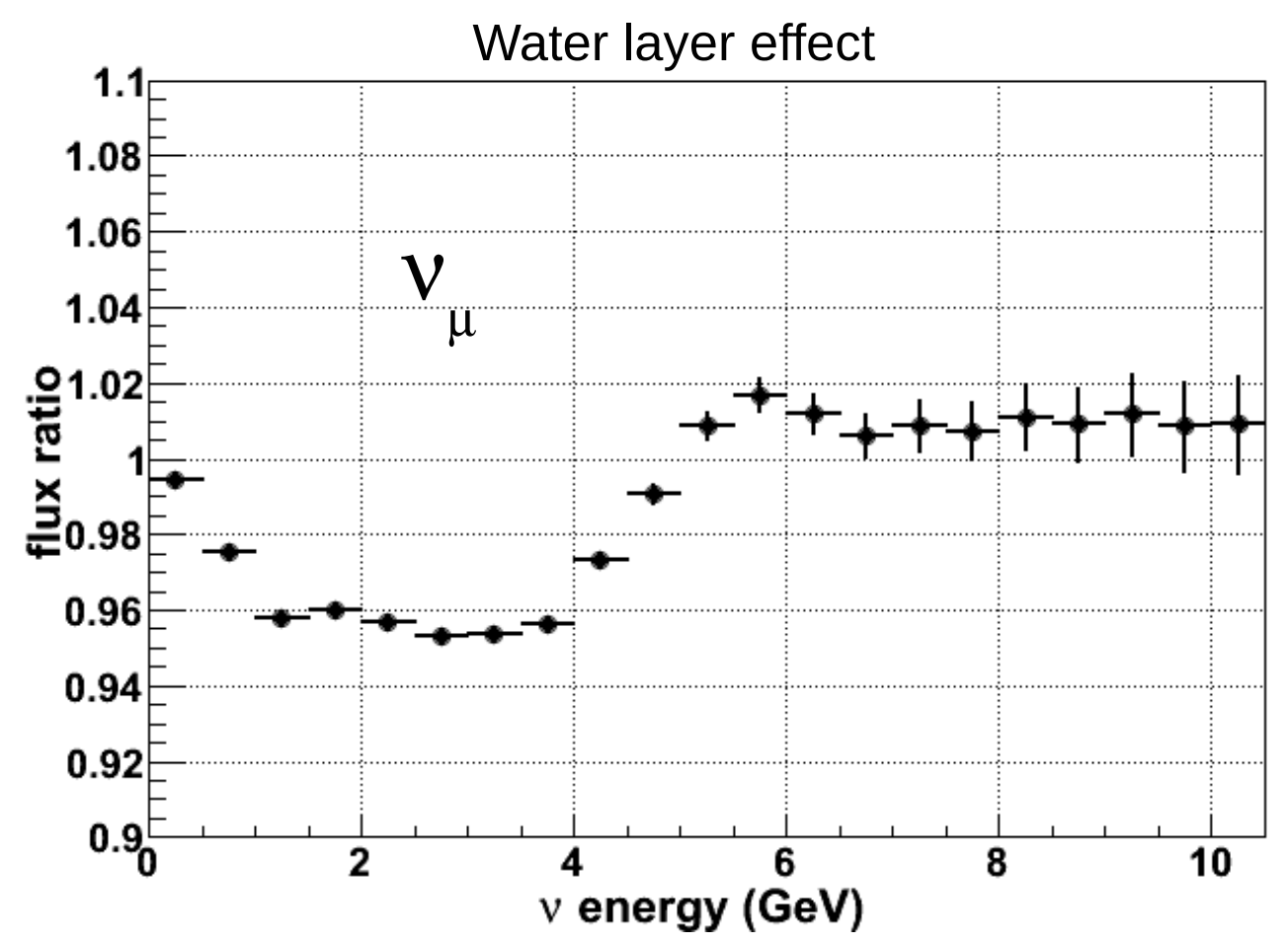

FIG. 2.25: Effect of $1 \mathrm{~mm}$ water layer around the Horn 1 inner conductor in the $\nu_{\mu}$ flux at MINERvA in the LE010z185i configuration. 


\section{CHAPTER 3}

\section{Overview of neutrino flux determination strategies}

As was described in Chapter 2, there are two primary sources of uncertainties in NuMI and in most conventional neutrino beams. One of them comes from the incomplete knowledge of the physics of the hadronic interactions that makes the simulation rely on phenomenological models, and the other one comes from some geometrical unknowns associated with the focusing process, relevant in some neutrino energy ranges. This chapter is dedicated to presenting different approaches to determine the neutrino flux in a conventional neutrino beam context as well the MINERvA strategy. We do not pretend to present the strategies of all experiment's approaches in detail. We want to place the MINERvA efforts as a part of a bigger effort in the experimental neutrino community.

We can distinguish two kind of procedures: those that determine the flux as an external input (i.e., without any intervention of the detector in where they are trying to find the flux) and other procedures that require the use of some data taken in the same detector for which we are trying to calculate the flux, but applying a 
criteria to deconvolute the flux (a "standard candle" process) that produced the data (this is called in situ). For the former procedure, we show the hadron production constraint as an example (Section 3.1). For the later one, we present three examples: multi-beam fitting (Section 3.2), the low-nu method (Section 3.3) and the neutrino - electron scattering constraint (Section 3.4). Other procedures are not considered here (like the use of muon monitors [30]) because they are not within the scope of this thesis.

\subsection{Using external data}

To apply a constraint to the hadron production in the cascade that leads to a neutrino in the flux simulation, we need to have some knowledge about the number of hadrons produced in the interactions and their kinematic distributions.

During the past few decades an extensive program has been running in many experiments to measure cross-sections of hadron-nucleus collisions in the non-perturbative QCD regime. This has been useful to understand the effects of the intra-nuclear interactions and to test model hypotheses. In recent years, new experiments have been running, motivated by the search for the quark-gluon plasma and to help to improve the simulations of neutrino fluxes and cosmic ray air showers. Sections 4.3 to 4.7 contain lists of some of these experiments in the context of our hadron production correction procedure.

A complete and model independent knowledge of all interactions in this regime would mean having measurements of interactions of every particle with all incident energies on all possible targets. Even though there are many datasets available, there are still a number of areas that lack the data coverage needed for a specific conventional neutrino beam. Recent experiments dedicated to measuring the production of hadrons from proton beams incident on thin (few percentage of interaction 
length) Be, C, Al targets. These experiments were optimized to measure hadrons which make a large contribution to the neutrino flux in running experiments like MiniBooNE, T2K and MINOS. Other efforts involve making direct measurements on the same target (or a replica) used to produce a neutrino beam. The idea is to use all data available, incorporating their uncertainties and making well-founded arguments to extrapolate to areas not covered by data.

The MiniBooNE experiment used data from the HARP experiment at CERN to determine their flux. HARP measured the cross-sections of protons and pions with momenta ranging from $1.5 \mathrm{GeV} / \mathrm{c}$ to $15 \mathrm{GeV} / \mathrm{c}$ and interacting in different target materials like beryllium, carbon, aluminum ([31], [32], [33], [34], and [35]) and also measured the yields of particle production in a MiniBooNE replica target ([36]) with a good forward coverage.

The Booster beamline (see Section 2.2.1) collides $8 \mathrm{GeV}$ protons into a $71.1 \mathrm{~cm}$ long Be target [37], and, in conjunction with a one magnetic horn system, creates an on-axis muon neutrino beam peaked at $500 \mathrm{MeV}-600 \mathrm{MeV}$. Figure 3.1a shows the kinematic distribution of $\pi^{+}$at the target that contributes to the $\nu_{\mu}$ flux at the MiniBooNE detector. The figure was taken from the MiniBooNE flux prediction paper [38]. The black box represents the HARP coverage of $\pi^{+}$produced from proton - Beryllium interactions at $8.9 \mathrm{GeV}$ [34] that was used to constrain the flux. Most of the pions relevant to MiniBooNE are constrained by the HARP data. More details about other datasets employed by MiniBooNE and their procedure can be found in their flux paper [38].

Another example is the T2K flux constraint using NA61 data. Their muon neutrino beam is peaked at $0.6 \mathrm{GeV}$ and is produced by colliding protons at $30 \mathrm{GeV}$ with a $91.4 \mathrm{~cm}$ long graphite target [39]. NA61 measured the cross-sections of protons interacting at $30 \mathrm{GeV}$ in carbon nuclei ([40] and [41]) and a T2K replica target ([42]). Figure 3.1b shows the $\pi^{+}$kinematic distribution at the target that con- 
tributes to the muon neutrino flux at the T2K far detector (Super-Kamiokande). The area enclosed by the black lines corresponds to the NA61 $\pi^{+}$data coverage that was used in the flux calculation [39] showing that most of pion production is able to be constrained. A complete description of their procedure and all datasets used can be found in their flux paper [39].

\subsection{Using a multi-beam fitting}

As explained in Section 2.3, changing the beamline configuration may change the neutrino spectra. This allows us to relate, in each configuration in the simulation, the neutrino yield in one energy bin and a kinematic region of the hadrons produced in the target. Using the data measured in these different configurations, we can attempt to find the hadron yields from the target that make data and simulation agree for all configurations considered. This is an example of an "in situ" procedure.

MINOS followed this strategy ${ }^{1}$ using data from different beam configurations at their Near Detector, adjusting parametrized yields of the hadrons that exit the target [25]. The functional form used was similar to the BMPT parametrization [43]. Their goal was to find the best extrapolation of the flux at the Near Detector to the flux at the Far Detector [5] for their oscillation parameter search. In the introductory chapter, we used Figure 1.3 to highlight the importance of the flux determination. That figure shows how the $\mathrm{F} / \mathrm{N}$ ratio changes due to the beam fit procedure.

The result of MINOS's beam fit procedure is shown in Figure 3.2. The beam configurations have the same convention as we explained in Section 2.3 where the data and simulation before tuning and the simulation after the tuning are represented by dots, a gray line and a black line, respectively. The effects on the event

\footnotetext{
${ }^{1}$ In addition to the hadron production, MINOS also adjusted some beam focusing parameters.
} 


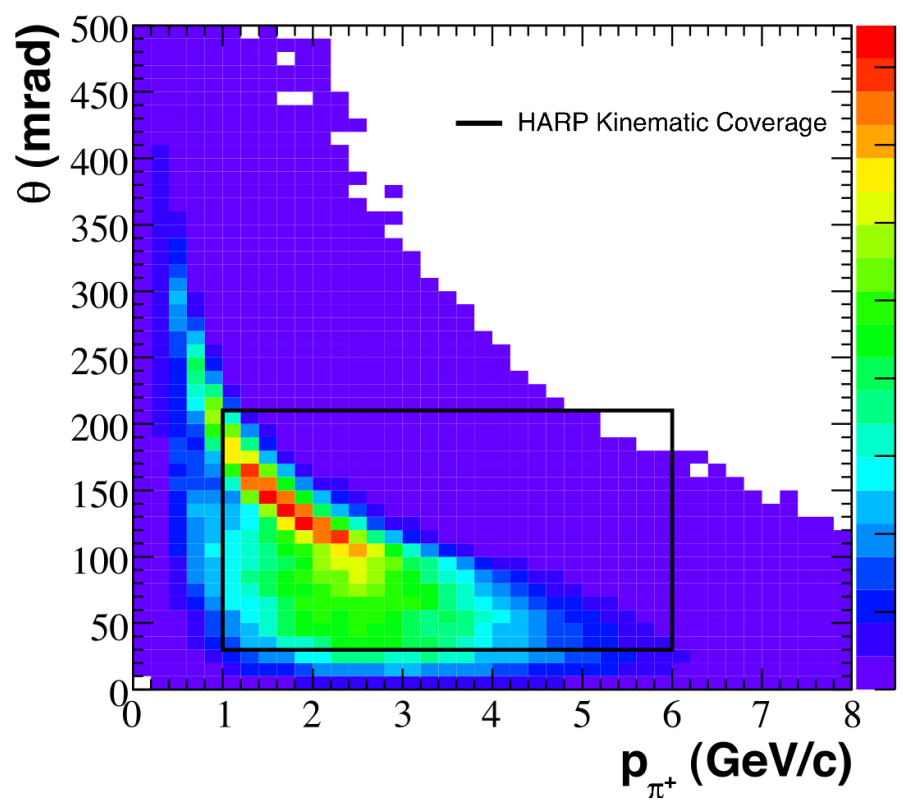

(a) $\pi^{+}$neutrino parents in MiniBooNE [38]. The black box enclosed the data coverage of HARP data of $\pi^{+}$produced in proton - beryllium interactions at $8 \mathrm{GeV}$. Plot taken from [34].

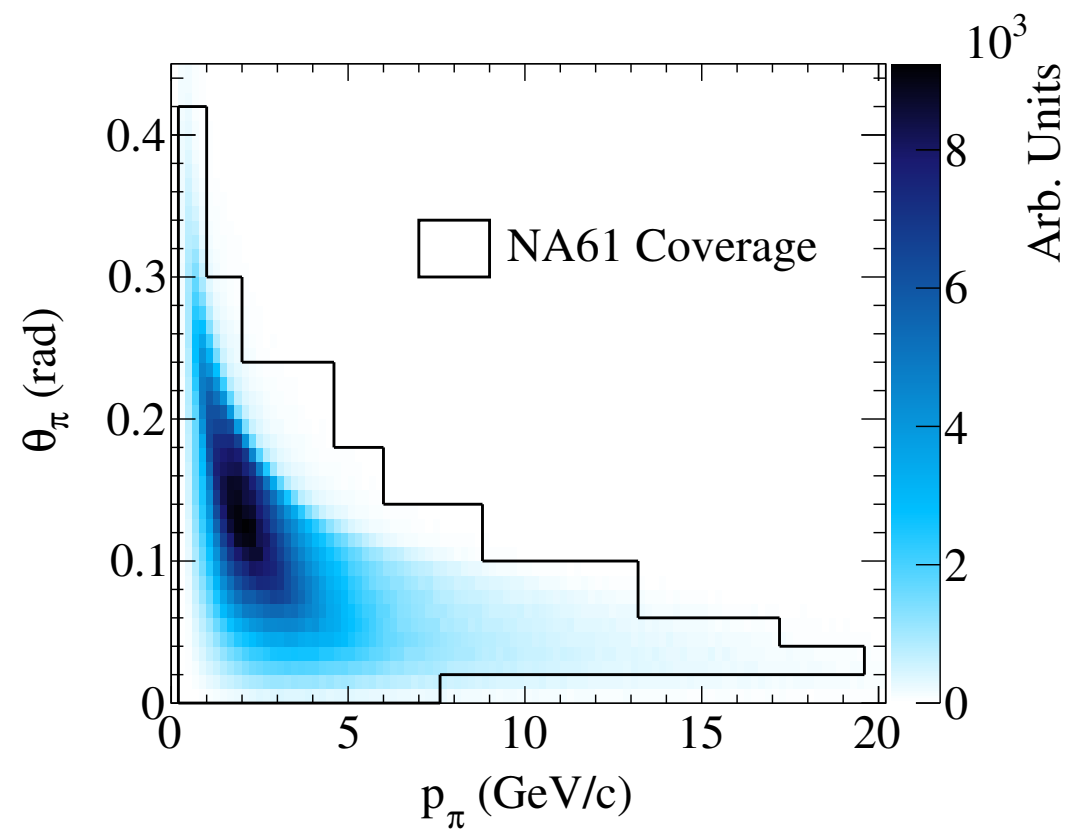

(b) $\pi^{+}$neutrino parents in T2K [39]. The area enclosed by black lines corresponds to the data coverage of NA61 $\pi^{+}$produced in proton - carbon interactions at $30 \mathrm{GeV}$. Plot taken from [40].

FIG. 3.1: Examples of hadron production experiments for neutrino flux (HARP and NA61). 
rates MINOS Near Detector can be seen in the ratios.

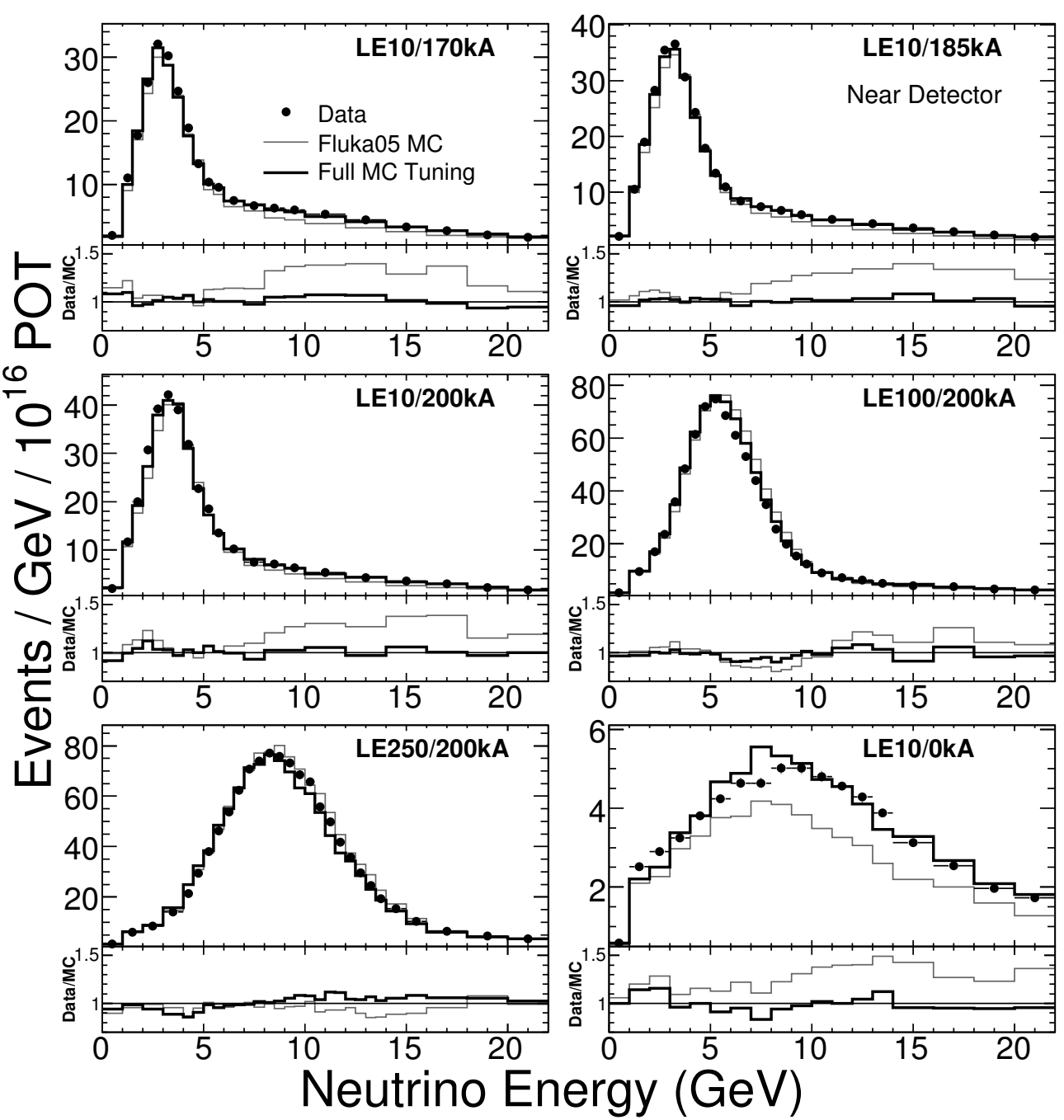

FIG. 3.2: Results of the multi-beam fitting procedure followed by MINOS to correct the NuMI flux in different configurations. Lower plots show ratio of data to MC simulation. Plot taken from [44].

\subsection{Low-nu method}

The differential cross-section of the neutrino-nucleon charged current scattering with respect to the recoil energy $(\nu)$ can be expressed as a combination of integrals 
over structure functions $(\mathrm{A}, \mathrm{B}$ and $\mathrm{C})$ and the ratio $\nu / E_{\nu}$, where $E_{\nu}$ is the neutrino energy:

$$
\frac{d \sigma}{d \nu}=A\left(1+\frac{B}{A} \frac{\nu}{E}-\frac{C}{A} \frac{\nu^{2}}{E^{2}}\right)
$$

The cross-section becomes independent of the neutrino energy as the ratio between the recoil energy and the neutrino energy goes to zero $\left(\nu / E_{\nu} \rightarrow 0\right)$. See [45] for more details. The energy spectrum of events with small $\nu / E_{\nu}$ will approximate the energy dependence of the flux (the flux shape). An additional normalization to external data is required (for instance, a cross-section measurements) so the absolute flux can be determined.

The method was developed by the $\mathrm{CCFR} / \mathrm{NuTeV}$ collaboration to determine the neutrino flux at energies greater than $30 \mathrm{GeV}$ and it used this flux to calculate the neutrino cross-sections [46] on iron. Some efforts have been made to extend the procedure to lower energies by MINOS and MINERvA. MINOS used this procedure to calculate the muon neutrino and antineutrino inclusive cross-section on iron $[47$, 44].

Recently, MINERvA also estimated the flux in the LE010z185i configuration using the low-nu method [48]. There are two challenges associated with the estimate and the procedure used to make it:

- To find a correction for finite $\nu$ values. MINERvA uses GENIE to compute corrections and the uncertainty on them.

- To accurately reconstruct the hadronic recoil energy.

The low-nu MINERvA results are used as a check of the flux in this thesis and additional details are shown in Section 6 . 


\subsection{Neutrino - electron scattering constraint}

Another in situ procedure uses elastic neutrino scattering on electrons as a standard candle. Standard electroweak theory predicts this cross-section precisely since ony leptons are involved in the $\nu e \rightarrow \nu e$ process, as shown in Figure 3.3.
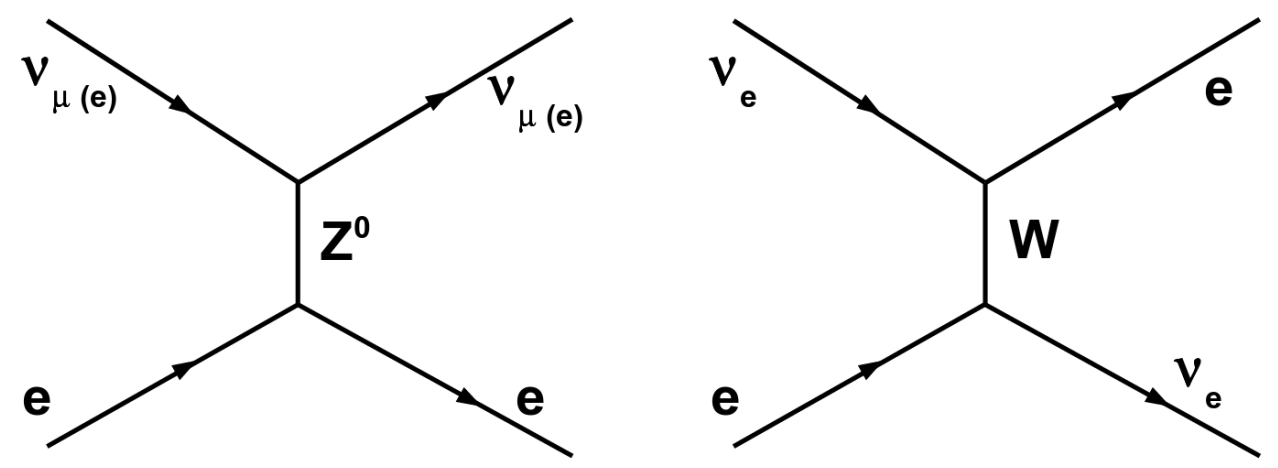

FIG. 3.3: neutrino - electron scattering Feynman diagram.

The $\nu e \rightarrow \nu e$ scattering process produces a single forward electron and a neutrino. The electron can be easily identified with a high resolution detector like MINERvA. However, the cross-section is approximately 2000 times smaller than the neutrino - nucleon cross-section and is thus statistically limited. The neutrino produced escapes the detector and it is not possible to reconstruct the incident neutrino energy. However, it serves as a flux normalization since the number of interactions can be counted and we can then determine the number of neutrinos that passed through the detector.

Recently, MINERvA studied these events [49] and this is applied as an additional constraint for the flux determined in this thesis. More details are provided in Section 6 . 


\subsection{Minerva strategy}

MINERvA has adopted a multi-layer approach to predict the NuMI flux that involves all the procedure described before: the use of external hadron production data (Section 3.1), the low-nu method (Section 3.3), the neutrino-electron constraint (Section 3.4) and a multi-beam fitting of the hadron production (Section 3.2).

The MINERvA flux prediction has been constantly updated, motivated by several factors including the incorporation of new hadron production data, a better understanding of the effect of flux systematics, and geometrical improvements in the NuMI simulation. The first two flux versions, called Generation 0 and Generation 1, have been used as the standard flux in the current MINERvA publications (see Table 5.1). The most updated flux version - Generation 2 - will be used in all upcoming MINERvA papers and it is the basis of the final results in this thesis. The details about every generation will be provided in the subsequent chapters.

The MINERvA strategy is the following:

- The foundation is formed from constraining hadron production with external measurements on thick and thin targets (see Chapter 4). The use of independent data gives us the chance to have different predictions.

- Beam optics uncertainties are incorporated by propagating errors in the alignment of beamline elements and the horn's geometry and magnetic field (see Section 2.5). The flux determined by hadron production constraints plus the focusing uncertainties is an a priori flux.

- In-situ measurements are then incorporated atop to this foundation. These consist of:

- The rate of charged current muon neutrino and antineutrino scattering events with low energy transfer to the target for which the flux shape is known (see 
Section 3.4). This is used to study the consistency of our flux predictions.

- Neutrino-electron scattering events for which the cross-section is known (see Section 3.4). This is used to reduce the flux uncertainty.

- The Multi-beam fitting technique had been followed early on before MIPP NuMI data was published and it is not used in this thesis. However, it served to check sources of MINERvA reconstruction issues.

One criteria used to design the MINERvA strategy has been the desire to develop flux calculating software tools that can be shared with other NuMI experiments. That is the reason for the PPFX package (see Section 4.8).

The next chapter explains the hadron production correction procedure we followed and the incorporation of the datasets we used in the context of the Generation 2 flux version ${ }^{2}$. See Section 4.3 to 4.7 .

The details of Generation 0 and Generation 1 are postponed to Chapter 5 when a historical review is made and the a priori LE flux results of every generation are presented. Chapter 6 is dedicated to the incorporation of in situ measurements and to compare Generation 0-1 to Generation 2 to update the MINERvA results already published. In Chapter 7, we will extend our procedure to ME flux and to other detectors.

\footnotetext{
${ }^{2}$ Explaining the dataset incorporation in the context of Generation 2 seems more convenient since it is the base of the result of this thesis.
} 


\section{CHAPTER 4}

\section{Correcting the simulation using hadron production data}

The non-perturbative QCD nature of the hadronic interactions in any conventional neutrino beam require the use of phenomenological models in the simulation. In principle, if we have enough experimental data to constrain the model, the flux can be determined and its uncertainty can be well understood. As was explained in the introductory chapter, knowing the flux is important in neutrino oscillation studies and it is crucial for neutrino cross-section measurements. How can the NuMI beam flux simulation be constrained using hadron production data? This chapter addresses this question by developing the MINERvA strategy that was introduced in Section 3.5.

This chapter is structured in three main parts. The first one establishes some common definitions (Section 4.1) and explains in detail the procedure we followed to correct the hadron production (Section 4.2). In the second part we survey all existing relevant data for NuMI and describe their implementation in the context of Generation 2 flux version (Sections 4.3 to 4.7). The last part is dedicated to 
introducing the tool we developed to handle the data in a computational framework: PPFX (Section 4.8).

\subsection{Definitions}

Before explaining our procedure, it is worthwhile to state some basic definitions that we use in this chapter and throughout the rest of the thesis.

\subsubsection{Total cross-section}

In hadron - hadron interactions $(\mathrm{h}+\mathrm{h})$, the total hadronic cross-section $\left(\sigma_{\text {total }}\right)$ can be divided in two components, one elastic $\left(\sigma_{\text {elastic }}\right)$ and the other one inelastic $\left(\sigma_{\text {inelastic }}\right)$ :

- Elastic component $(h+A \rightarrow h+A)$ : the nucleus remains intact after the interaction. This implies that neither mesons nor nucleons are ejected from the nucleus.

- Inelastic component $(h+A \rightarrow+$ mesons + fragments of nucleus $)$ : the nucleus is broken and new particles are created.

However, when we consider hadron - nucleus interactions $(\mathrm{h}+\mathrm{A})$ another component arises: "quasi-elastic":

- Quasi-elastic component $(h+A \rightarrow+\mathrm{p}+$ fragments of nucleus): the nucleus is broken but no new particles (mesons) are created. It is characterized by a fast nucleon in the final state (typically, $x_{F}>0.95$ ) and one or more slow nucleus fragments.

In the context of $\mathrm{h}+\mathrm{A}$ interactions, we define the absorption cross-section

$\left(\sigma_{\text {absorption }}\right)$ as the sum of the quasi-elastic and inelastic components. Then, the total 
hadronic cross-section is:

$$
\sigma_{\text {total }}=\sigma_{\text {elastic }}+\underbrace{\sigma_{\text {inelastic }}+\sigma_{\text {quasi-elastic }}}_{\sigma_{\text {absorption }}}
$$

\subsubsection{Differential cross-section}

The Lorentz invariant differential cross-section is defined as:

$$
f=E_{s} \frac{d^{3} \sigma}{d p^{3}}
$$

where $E_{s}$ and $p$ are the energy and momentum of the particle produced in the interaction.

The approximate scaling variable "Feynman-x" ([50] and [51]) is defined as:

$$
x_{F} \equiv \frac{p_{\|}^{*}}{P_{\|}^{*}(\max )} \simeq \frac{2 p_{\|}^{*}}{\sqrt{s}}
$$

where $(*)$ indicates that the variable is calculated in the center of momentum system,

$p_{\|}^{*}$ is the longitudinal momentum of the produced particle and $P_{\|}^{*}(\max ) \simeq \sqrt{s} / 2$ is the maximum momentum allowed ( $\sqrt{s}$ is the energy of the center of mass). Feynman speculated that the cross-sections of inclusive high energy hadronic collisions would have an energy-independent scaling behavior when expressed in terms of $x_{F}$.

\subsection{Procedure}

This section is dedicated to explaining in detail the procedure we followed to make the hadron production correction.

Each interaction in the neutrino's ancestry chain is corrected with a weighting factor computed from yields or invariant differential cross-sections: 


$$
c_{i}=\frac{N_{i}^{\text {data }}}{N_{i}^{M C}}
$$

The factor $c_{i}$ depends on the identities of the projectile, target, and produced hadron as well as the initial and final state kinematics. Here "i" denotes the initial and final state information, and it is generally a particular bin in a hadron production dataset. In that case we have an uncertainty $\sigma_{i}$ and a covariance with other bins $\mathrm{j}, V_{i j}$. Even in the case that the interaction in question is not covered by a hadron production dataset, we still assign $c_{i}, \sigma_{i}$, and $V_{i j}$ factors using our best

judgment. $N_{i}^{M C}$ is either taken directly from the MC or computed by simulating the interactions in question to produce a yields table or an invariant cross-section.

We refer to each $c_{i}$ as the "central value" estimate for the interaction. In Section 4.2.5 we will describe the way in which the $V_{i j}$ are used to vary the weighting factors to propagate uncertainties.

In addition to the weights applied to each interaction we also account for attenuation of particles in the beamline, inserting correction factors which depend on the absorption cross-section in data and MC.

\subsubsection{Attenuation Corrections}

When a beam particle passes through a volume, the interaction rate depends on the cross-section and the amount of material traversed. The probability $P(r)$ of a particle not interacting while crossing a distance $r$ is given by:

$$
P(r)=e^{-r N_{A} \rho \sigma}
$$

Where $N_{A}$ is the Avogadro number, $\rho$ is the nuclei volume density and $\sigma$ is the absorption cross-section per nucleus. 

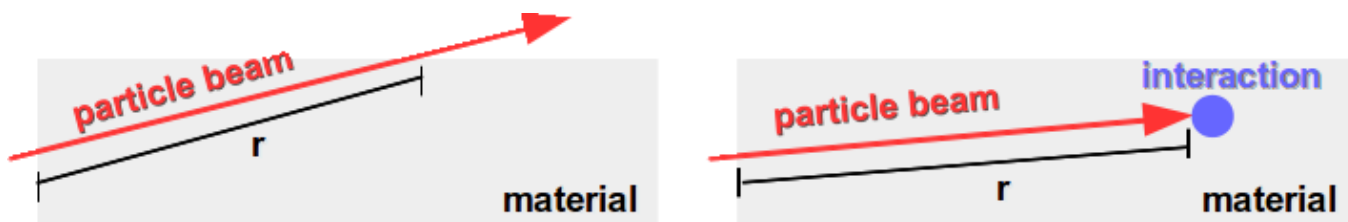

FIG. 4.1: Particle beam traversing a volume. Left side: particle leaving the volume without interacting. Right side: particle interacting in the volume.

If an interaction does not happens (see Figure 4.1, left side), this correction is applied to the probability that the particle survived:

$$
c(r)=e^{-r \frac{N_{A} \rho\left(\sigma^{d a t a}-\sigma^{M C}\right)}{A}}
$$

The material traversed $r N_{A} \rho$ is independent of the specific material and it can be expressed as $\mathrm{mol} / \mathrm{cm}^{2}$. The correction is shown in the Figure 4.2 considering a cross-section disagreement up to $\pm 20 \mathrm{mb}$ and up to $10 \mathrm{~mol} / \mathrm{cm}^{2}$ of material.

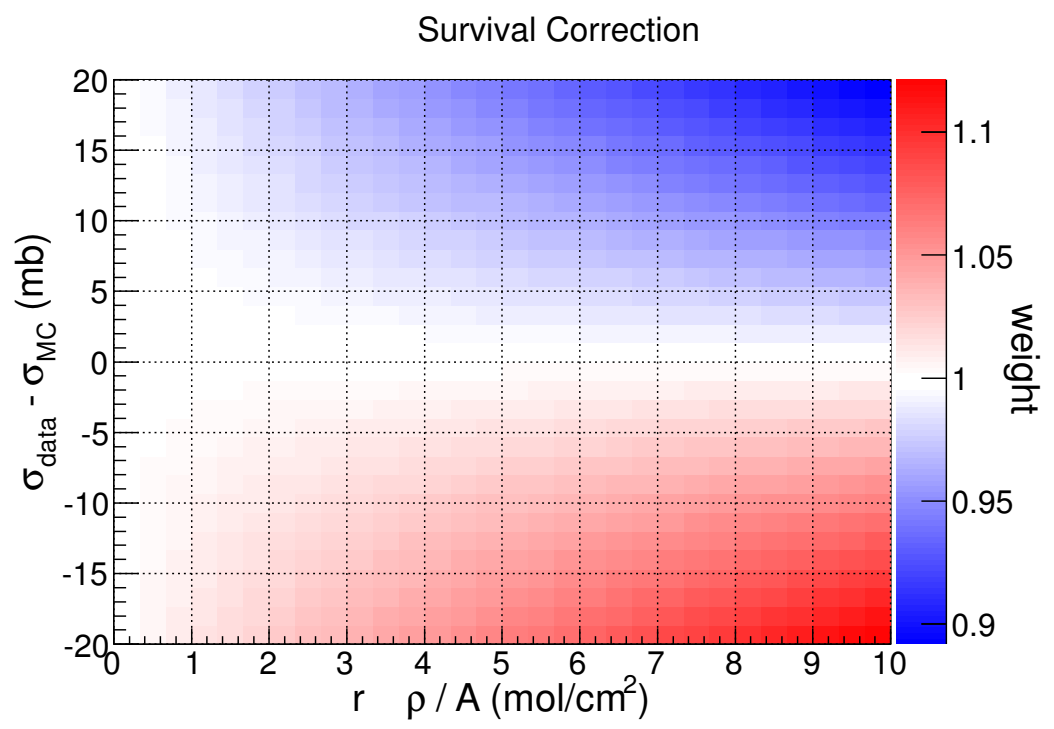

FIG. 4.2: The survival correction.

There is no correction to apply when the agreement between data and MC is perfect (the factor in this case is 1). When the material traversed is very small the 
factor is also close to 1 , for example, when the particle is created on the edge of a volume. If the $\mathrm{MC}$ overestimates the cross-section, less particle beam survives in the simulation and the correction goes up and increases for increased material traversed. Conversely, if the MC underestimates the cross-section, more particle beam survived in the simulation and the correction goes down and further decreases for increased material traversed.

If an interaction happens in a volume (see Figure 4.1, right side), the correction is applied to the probability that the particle survived up to the interaction point:

$$
c(r)=\frac{\sigma^{d a t a}}{\sigma^{M C}} e^{-r \frac{N_{A} \rho\left(\sigma^{d a t a}-\sigma^{M C}\right)}{A}}
$$

Now, the attenuation correction also depends on the data over MC crosssections ratio. The Figures 4.3 and 4.4 show this correction for two fixed MC cross-section values: $\sigma=193.6 \mathrm{mb}$ (this corresponds to $\pi$ on $C$ at $7 \mathrm{GeV}$ ) and $\sigma=368 \mathrm{mb}(\pi$ on $A l$ at $7 \mathrm{GeV})$ respectively. As before, in a perfect data-MC crosssection agreement the correction would be 1. Interactions are more (less) likely to occur in the simulation if the MC overestimates (underestimates) the cross-section and this effect competes with the survival portion of the function creating the patterns we see in the figures.

\subsubsection{Particle Production}

Thin target experiments tend to release their data as invariant double differential cross-sections $f$ in terms of Feynman-x $x_{F}$ and transverse momentum $p_{T}$. As was mentioned in Section 4.1.2, Feynman-x is an approximate scaling variable and it lets us extrapolate a cross-section taken at one energy to another one in inelastic collisions. When scaling data like NA49 that was taken at $158 \mathrm{GeV}$ (see Section 4.5.1) we use FLUKA $[52,53]$ to remove the residual energy dependence and to 


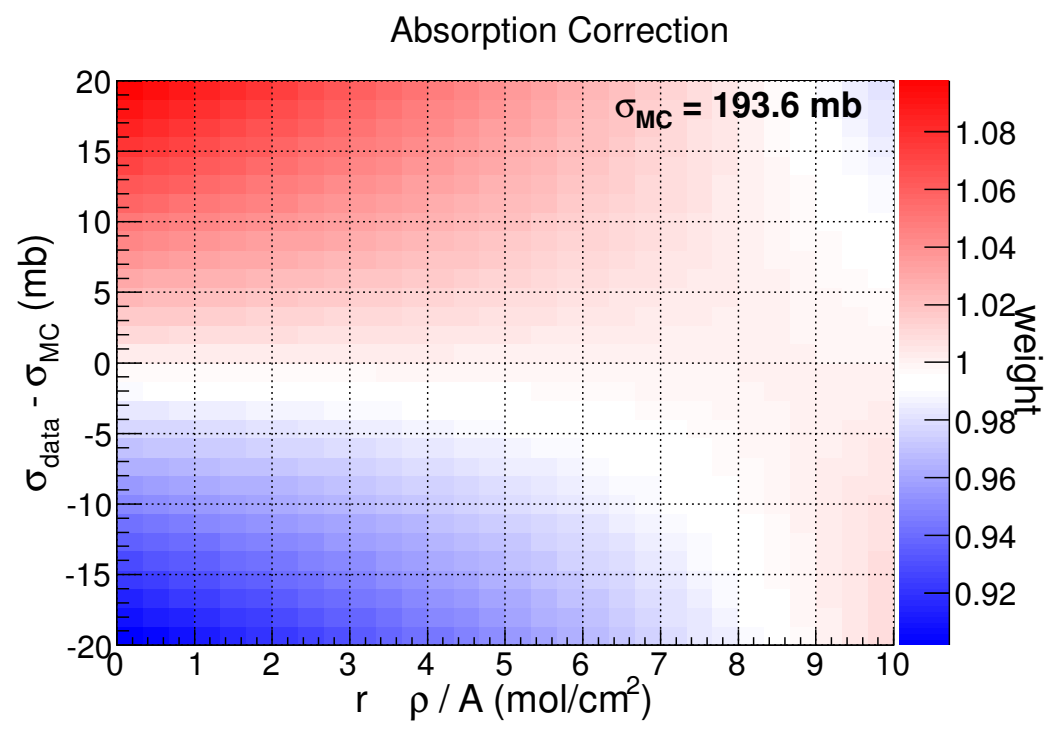

FIG. 4.3: Attenuation correction when an interaction happened for $\sigma_{M C}=193.6 \mathrm{mb}$ (this corresponds to $\pi$ on $C$ at $7 \mathrm{GeV}$ ).

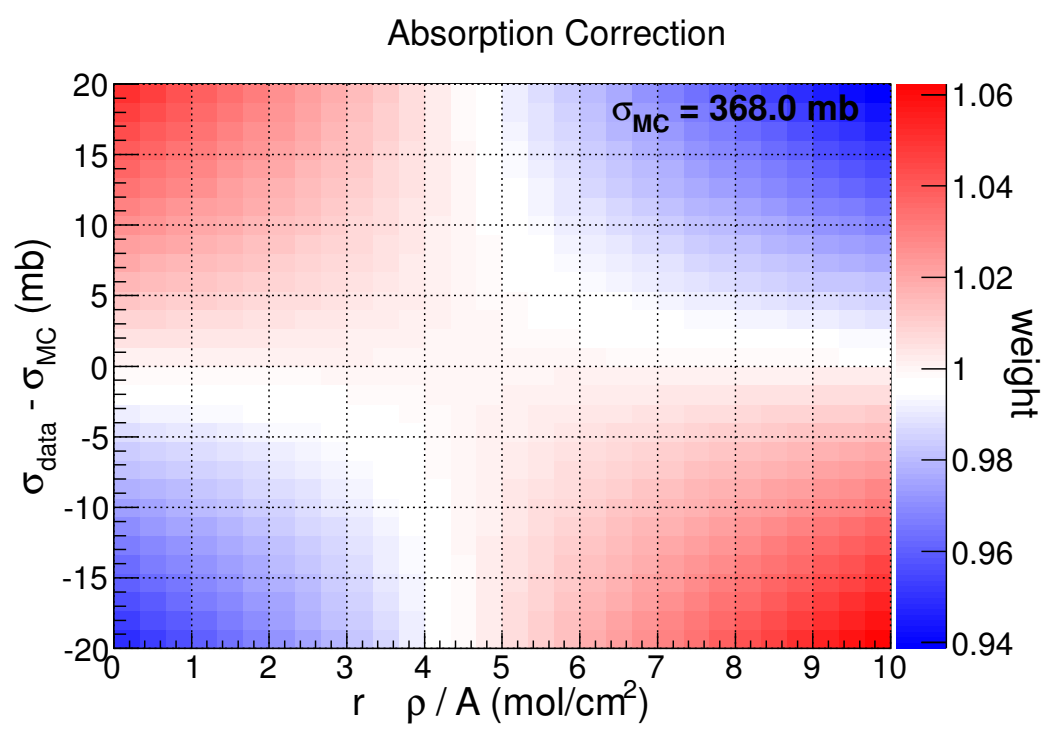

FIG. 4.4: Attenuation correction when an interaction happened for $\sigma=368 \mathrm{mb}$ (this corresponds to $\pi$ on $A l$ at $7 \mathrm{GeV}$ ). 
scale to $12-120 \mathrm{GeV}$ [54] that is the range relevant for NuMI. This "energy scaling" correction is shown in Figure 4.5 for four $x_{F}$ ranges for positive pions generated in proton on carbon collisions. For typical $p_{T}$ values $(\sim 300 \mathrm{MeV} / \mathrm{c})$ the correction at $120 \mathrm{GeV}$ is very small (less than $1 \%$ ) but it can be up $10 \%$ when the energy of the incident proton goes down to $12 \mathrm{GeV}$.
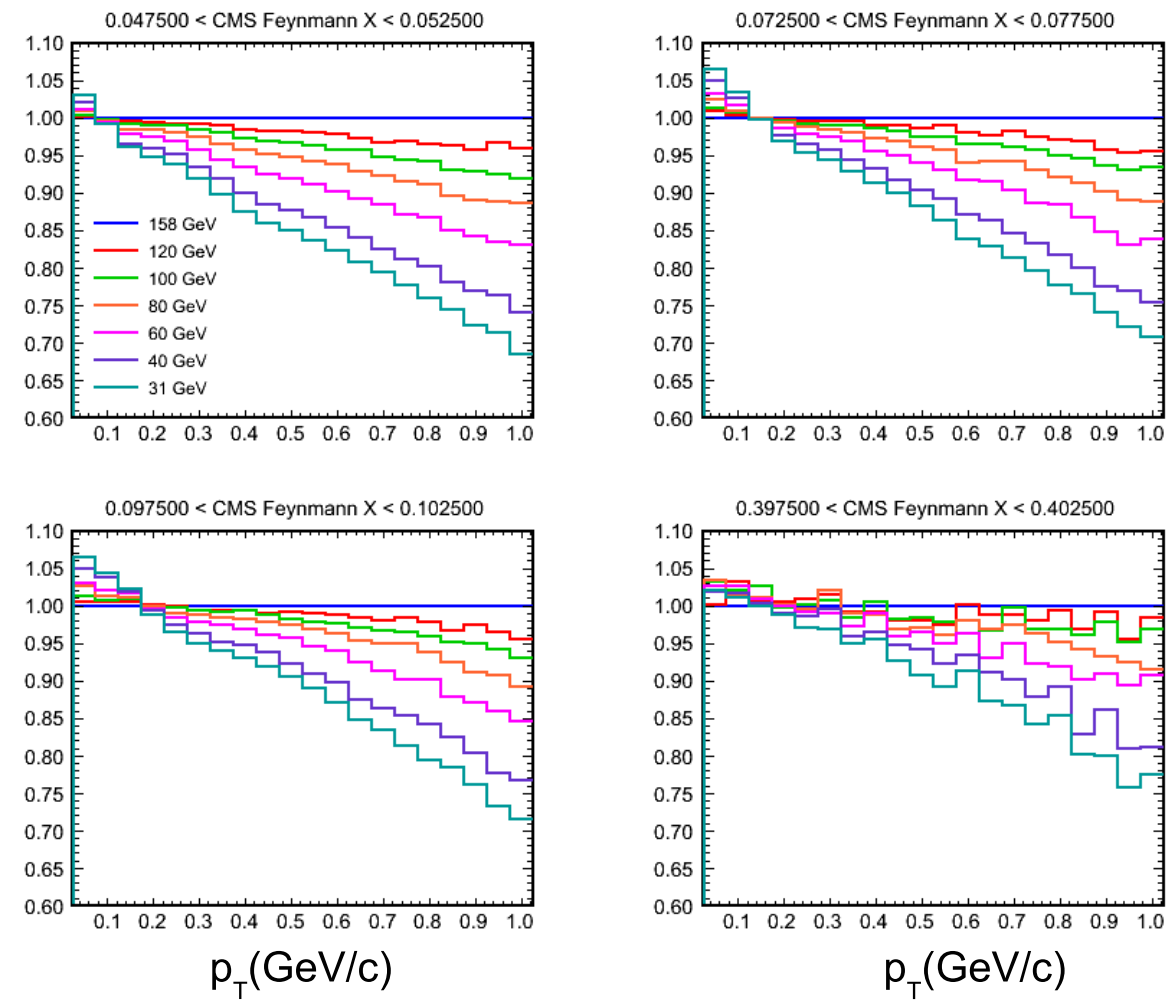

FIG. 4.5: FLUKA energy scaling correction for invariant cross-section data taken at $158 \mathrm{GeV}$ to lower energy down to $31 \mathrm{GeV}$. This is split in four $x_{F}$ ranges.

The formula to be applied becomes:

$$
c\left(x_{F}, p_{T}, E\right)=\frac{f_{\text {Data }}\left(x_{F}, p_{T}, 158 G e V\right) \times \operatorname{scale}\left(x_{F}, p_{T}, E\right)}{f_{M C}\left(x_{F}, p_{T}, E\right)}
$$

The energy scaling correction has been calculated for the following incident energies: $\{12,20,31,40,60,80,100,110,120\} \mathrm{GeV}$ and the MC cross-sections $f_{M C}$ for $\{12,20,31,40,50,60,70,80,90,100,110,120\}$ GeV. A simple linear 
interpolation is applied for intermediate incident energies. In Figure 4.6 the blue line represents NA49 data integrated in $p_{T}$ and expressed in terms of the angle of the pion produced as in NA61. The green line shows the effect of applying the energy scaling factors and shows that it is in good agreement with NA61 (purple line), especially around the focusing peak as it is shown in a zoomed-in version in the right plots.

This prescription was checked by scaling NA49 pion production data at $158 \mathrm{GeV}$ to NA61 data taken at $31 \mathrm{GeV}$. The difference between the two was very small. The effect on the neutrino flux was calculated to be no more than $1 \%$ (see [55]). Figures 4.7 shows the fractional difference between NA49 and NA61, where $\Delta f\left(x_{F}, p_{T}\right)$ is the discrepancy between the value of scaled NA49 value at $31 \mathrm{GeV}$ and the corresponding NA61 value.

When an interaction happens in a volume where we are applying an attenuation correction, we have to modify Equation 4.8 to cancel the inelastic cross-section contribution in the Equation 4.3 to avoid a double correction:

$$
c\left(x_{F}, p_{T}, E\right)=\frac{f_{\text {Data }}\left(x_{F}, p_{T}, 158 G e V\right) \times \operatorname{scale}\left(x_{F}, p_{T}, E\right)}{f_{M C}\left(x_{F}, p_{T}, E\right)} \times \frac{\sigma_{M C}(E)}{\sigma_{\text {data }}(E)}
$$

On the other hand, thick target experiments release their data as yields of particle production when they leave the target ${ }^{1}$. The correction in this case is given by the ratio of the data over MC yields:

$$
\operatorname{correction}\left(p_{Z}, p_{T}\right)=\frac{n_{\text {Data }}\left(p_{Z}, p_{T}\right)}{n_{M C}\left(p_{Z}, p_{T}\right)}
$$

\footnotetext{
${ }^{1}$ Longitudinal dependence of the yields is sometimes provided like in Ref. [42].
} 


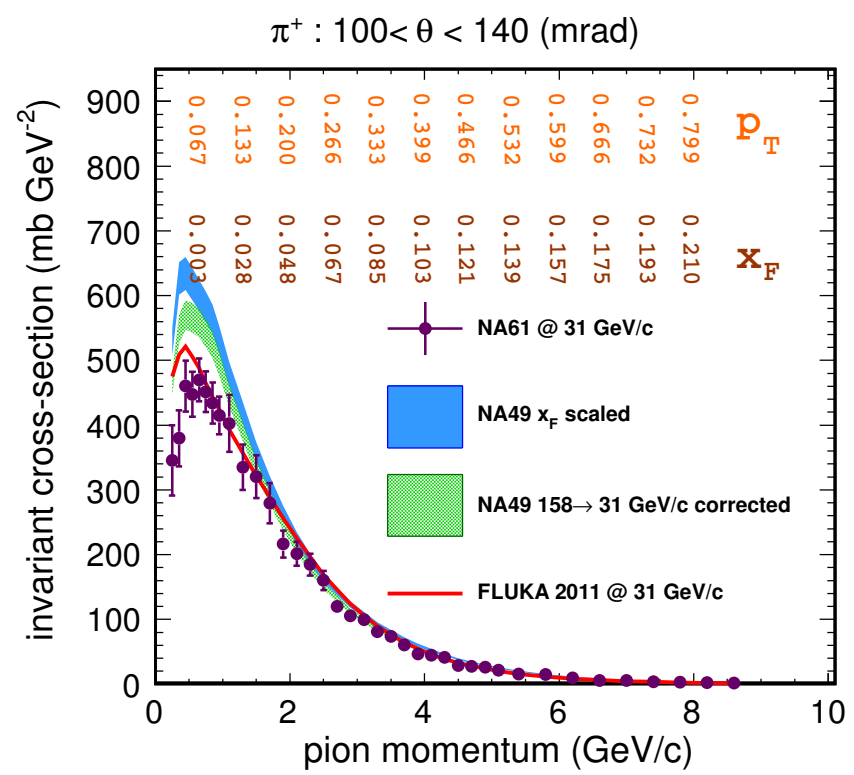

(a)

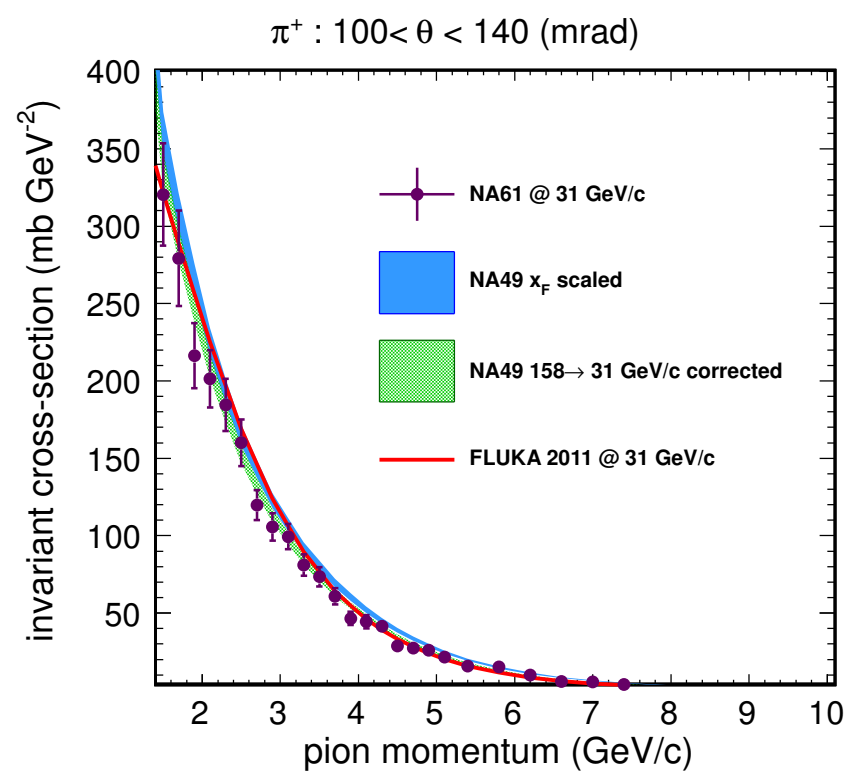

(b)

FIG. 4.6: Effect of the energy scaling for $\pi^{+}$produced on proton - carbon collisions. The corresponding $x_{F}$ and $p_{T}$ are indicated in the left side plot and the right plot is a zoomed version. 


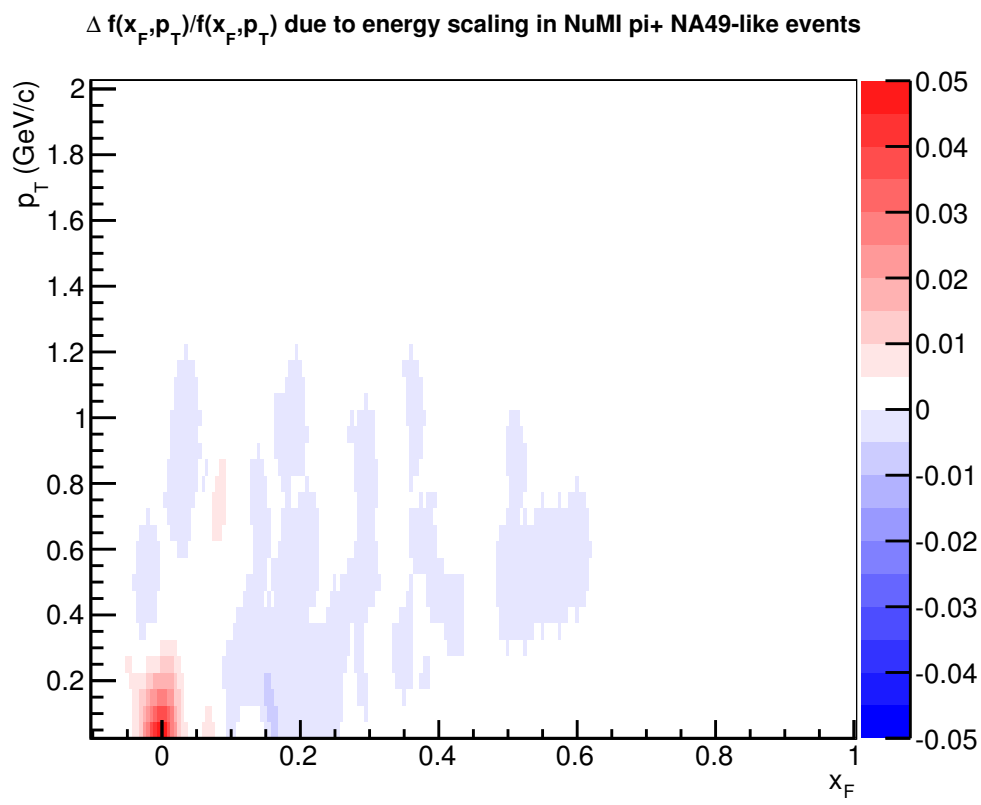

(a) $\pi^{+}$

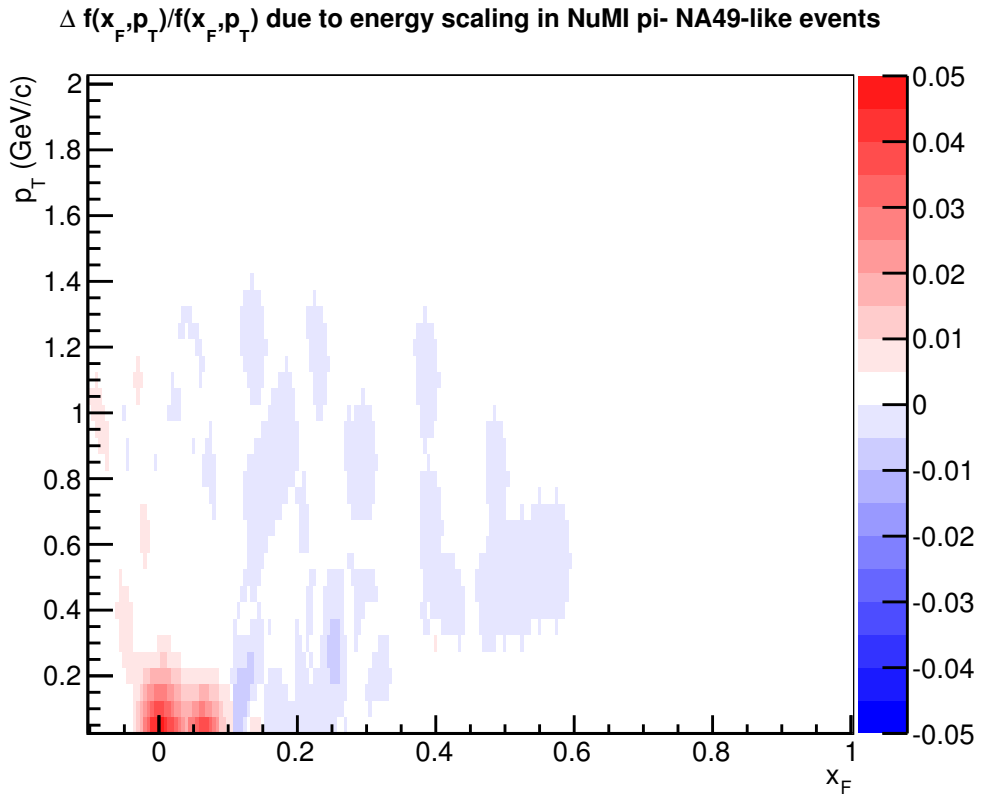

(b) $\pi^{-}$

FIG. 4.7: Fractional difference $\left(\Delta f\left(x_{F}, p_{T}\right) / f\left(x_{F}, p_{T}\right)\right)$ between NA49 and NA61 due to energy scaling. Plot taken from [55]. 


\section{Details of the target attenuation correction when using thick target data}

Applying both the thick target correction and the target attenuation correction gives a longitudinal target dependence to the hadron production correction. This is important since the hadrons that exit the target are focused differently depending on how they enter the horns. The challenge, as with the thin target correction, is to avoid double counting. The solution is not simple because there are many particles interacting or surviving in the target.

As we are going to see in Section 4.7, among all particles passing through the target, only the proton attenuation correction has its central value different than 1 and particularly, the primary proton beam has the most significant contribution in comparison to any secondary proton produced in the target. Pions and other particles do not change the central value.

Having this in mind, we assume that when a hadron that leaves the target is able to be corrected with thick target data, it is a good approximation to apply an attenuation correction to just the primary beam and consider that all other particle attenuation corrections are embedded in the thick target measurements.

Another simplification is that we consider only the longitudinal material traversed to calculate the attenuation correction since the primary proton momentum is high and almost parallel to the beamline, and the target length is also parallel to the beamline's longitudinal axis.

However, two modifications are needed to make this possible. The first one is

to calculate $n\left(p_{Z}, p_{T}\right)$ per interaction instead of per proton incident on the target by using a factor $y$, that is the fraction of the primary proton beam that interacts in the target. In this way, the beam that passes through the target without interacting can be treated independently. Equation 4.10 becomes: 


$$
c\left(p_{Z}, p_{T}\right)=\frac{n_{\text {Data }}\left(p_{Z}, p_{T}\right) / y_{\text {Data }}}{n_{M C}\left(p_{Z}, p_{T}\right) / y_{M C}}
$$

The second modification preserves the thick target data yield we are applying. The MC yield for a $\left(p_{Z}, p_{T}\right)$ bin can be expressed in terms of the longitudinal distribution $g(z)$ of the primary proton interaction positions in the target as:

$$
n_{M C}=\int_{0}^{z_{\max }} g(z) d z
$$

where $z_{\max }$ is the maximum longitudinal distance traveled by the proton in the target before interacting. After applying the attenuation correction $c_{a t t}$, the $n_{M C}$ becomes:

$$
n_{M C}^{\prime}=\int_{0}^{z_{\max }} g(z) c_{a t t}(z) d z
$$

And then,

$$
\begin{aligned}
n_{M C} & =n_{M C}^{\prime} \times \frac{n_{M C}}{n_{M C}^{\prime}} \\
& =\int_{0}^{z_{\max }} g(z)\left[c_{a t t}(z) \times \frac{n_{M C}}{n_{M C}^{\prime}}\right] d z
\end{aligned}
$$

The factor $n_{M C} / n_{M C}^{\prime}$ is calculated per bin and the final form of the Equation 4.7 is:

$$
c(r)=\frac{\sigma^{\text {data }}}{\sigma^{M C}} e^{-r \frac{N_{A} \rho\left(\sigma^{\text {data }}-\sigma^{M C}\right)}{A}} \times \frac{n_{M C}}{n_{M C}^{\prime}}
$$

\subsubsection{Extending data coverage}

Based on theoretical guidance, some extension of data is possible to different materials and different incident and produced particles. 
The first extension is "material scaling" and we are looking here for an uncertainty associated with using hadron production data taken on carbon in other materials. As was exhibited by Barton et al. [56], the invariant cross-section can be parametrized as:

$$
f\left(A, x_{F}, p_{T}\right)=\sigma_{0} A^{\alpha\left(x_{F}, p_{T}\right)}
$$

where $A$ is the mass number of the target nuclei different than hydrogen ${ }^{2}$. The parametrization is independent of the energy of the incident particle as the approximate scaling $x_{F}$ hypothesis suggests. Using this approach, the relation between the cross-sections of two materials can be express as:

$$
\frac{f\left(A_{1}, x_{F}, p_{T}\right)}{f\left(A_{2}, x_{F}, p_{T}\right)}=\left(\frac{A_{1}}{A_{2}}\right)^{\alpha\left(x_{F}, p_{T}\right)}
$$

MINERvA has followed the parametrization of $\alpha$ proposed by [56] and extended by $[43]$ :

$$
\alpha\left(x_{F}, p_{T}\right)=\left(a \cdot x_{F}^{2}+b \cdot x_{F}+c\right) \cdot\left(d \cdot p_{T}^{2}+e \cdot p_{T}+1\right)
$$

$\alpha\left(x_{F}, p_{T}\right)$ is found by doing an independent fit of the Skubic data [57] of invariant cross-sections of $K^{0}, \Lambda^{0}$ and $\bar{\Lambda}^{0}$ produced from proton interacting in various nuclei at $300 \mathrm{GeV}$. Figure 4.8 shows an example of the $\alpha\left(x_{F}, p_{T}\right)$ fit for neutral kaon production in proton interactions at $x_{F}=105 \mathrm{GeV} / \mathrm{c}$ and angle $=0.9 \mathrm{mrad}$. The same fit is made with all Skubic data, having $318 \alpha$ values in total.

The results are checked against $\pi^{ \pm}$and $K^{ \pm}$production in proton interactions to find the additional uncertainty to cover materials different than carbon with proton carbon data (more details an be found in [58]).

\footnotetext{
${ }^{2}$ The data fit using the Equation 4.17 in [56] excludes hydrogen.
} 


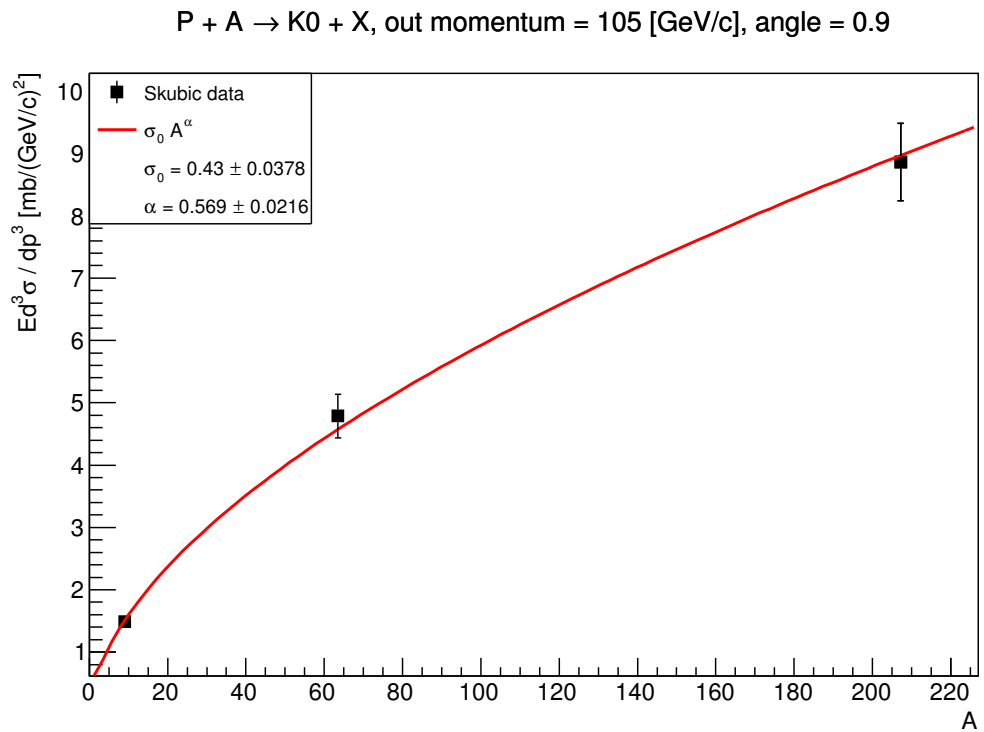

FIG. 4.8: Skubic data $\alpha\left(x_{F}, p_{T}\right)$ fit for neutral kaon production in proton interactions at $x_{F}=105 \mathrm{GeV} / \mathrm{c}$ and angle $=0.9 \mathrm{mrad}$. The same fit is made with all Skubic data. This is shown as an example. Plot taken from [58].

The additional uncertainties to extend data taken in carbon to other materials $\left(p_{T}\right.$ units are $\left.\mathrm{GeV} / \mathrm{c}\right)$ are in Table 4.1.

Another extension comes from the isoscalar nature of ${ }^{12} \mathrm{C}$ (the main material of the NuMI target). The isospin symmetry of deuterons establishes that $\sigma(p d \rightarrow$ $\left.\pi^{+} n d\right)=\sigma\left(n d \rightarrow \pi^{-} p d\right)$ and then we can infer that:

$$
\sigma\left(p C \rightarrow \pi^{ \pm} X\right)=\sigma\left(n C \rightarrow \pi^{\mp} X\right)
$$

This symmetry is used to determine the correction for hadron production in neutron carbon interactions and assume that the same correction is necessary for other materials than carbon. No additional uncertainty is added when using this extension. Since there is a small number of neutron interactions in the typical neutrino ancestry chain, and because we could not find any relevant data to test Equation 4.20. 
(a) $\pi^{+}$.

\begin{tabular}{|l|c|c|c|}
\hline$x_{F} p_{T}$ & {$[0,0.5]$} & {$[0.5,1]$} & {$[1,1.5]$} \\
\hline$[0,0.25]$ & 10 & 7.5 & 2.5 \\
{$[0.25,0.5]$} & 10 & 12.5 & 10 \\
{$[0.5,0.75]$} & 7.5 & 10 & 2.5 \\
\hline
\end{tabular}

(c) $K^{+}$.

\begin{tabular}{|c|c|c|c|}
\hline$x_{F} p_{T}$ & {$[0,0.5]$} & {$[0.5,1]$} & {$[1,1.5]$} \\
\hline$[0,0.25]$ & 10 & 7.5 & 2.5 \\
\hline$[0.25,0.5]$ & 10 & 12.5 & 10 \\
\hline$[0.5,0.75]$ & 7.5 & 10 & 2.5 \\
\hline
\end{tabular}

(b) $\pi^{-}$.

\begin{tabular}{|l|c|c|c|}
\hline$x_{F} p_{T}$ & {$[0,0.5]$} & {$[0.5,1]$} & {$[1,1.5]$} \\
\hline$[0,0.25]$ & 12.5 & 7.5 & 2.5 \\
{$[0.25,0.5]$} & 10 & 7.5 & 15 \\
{$[0.5,0.75]$} & 15 & 10 & 20 \\
\hline
\end{tabular}

(d) $K^{-}$.

TABLE 4.1: Additional uncertainties (\%) when extending data taken in carbon to other materials $\left(p_{T}\right.$ units are $\left.G e V / c\right)$.

The neutral kaons can be determined from charged kaons assuming isospin symmetry in a quark parton model [43]. If the number of valence quarks $u$ is the double of $d$ and $q_{s}=\bar{q}_{s}$ for $q=\{u, d, s\}$, then:

$$
N\left(K_{L(S)}^{0}\right)=\frac{N\left(K^{+}\right)+3 N\left(K^{-}\right)}{4}
$$

In this way a correction for neutral kaons for any material can be calculated when $K^{ \pm}$data is available and we also extend also this procedure to thin target data. Since the kaon data is dominated by statistical errors, no uncertainty is added from these assumptions.

\subsubsection{Procedure when there is no data}

We have two options in assigning an uncertainty to the hadron production when there is not clear theoretical guidance to extend the data coverage.

1. First we can examine the spread between different hadronic models. This was the 
original approach in MINERvA (see Sections 5.1 and 5.2) to handle interactions not covered by NA49. The differences between models could depend on the type of interactions. Then, to avoid underestimating the true flux differences predicted by each model, we categorized interactions not covered by NA49 and looked at the maximum difference between geant4 hadronic models for the following categories for each individual interaction that is relevant for the total neutrino flux and assumes this as the uncertainty. A complete list of categories and more details can be found in [59]. Examples of these categories are:

- $\pi^{+}$neutrino parents that were born in the target whose ancestry includes proton reinteraction in the target.

- $K^{+}$neutrino parents that were born inside the magnetic horns.

Figure 4.9 shows the predicted fluxes due to $\pi^{+}$neutrino parents born in the target whose ancestry involves a previous pion interaction from 5 geant 4 hadronic models. The spread in this case is $\sim 40 \%$ around the average value in the focusing peak.

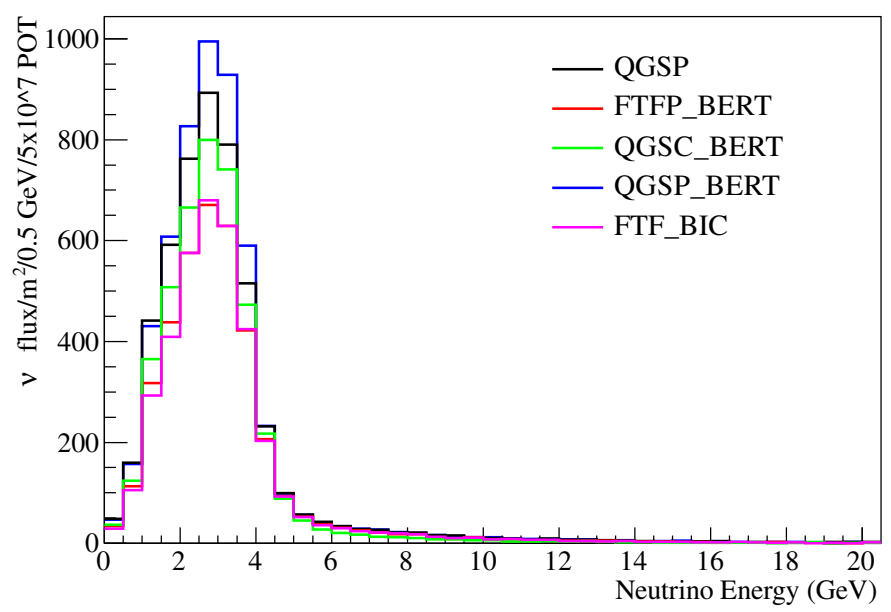

FIG. 4.9: Example of geant4 model spread: neutrino flux from $\pi^{+}$parents born in the target with a pion ancestry. 
The problem with this procedure is that it is hard to determine any internal correlation between models and we end up with the risk of underestimating the final flux error.

2. Second we apply a best guess uncertainty based by the agreement with other datasets. Our idea is that we are not totally blinded for interactions for which there is not data. The MC hadronic model we used (FTFP_BERT) is a microphysical model that applied QCD (in general, particle physics) first principles to all components in the interactions. Then we expect that the agreement (or disagreement) between data and MC for interactions that have data should be roughly the same as interactions not covered by data.

We notice three characteristics of the corrections when we look at the data:

- the corrections between different produced particles are not correlated.

- most of the correction values are in the range 0.6-1.4.

- the corrections depend on $x_{F}$ in a first order approximation, i.e., not $p_{T}$ dependence, since the neutrino energy is proportional to $x_{F}$.

Considering this, for the interactions where we do not find any data or extension to apply, we categorized those interactions in $4 x_{F}$ regions $(0-0.25,0.25-0.5,0.5-$ 0.75 and $0.75-1.0)$ per any combination of projectile and produced particle and assign $40 \%$ uncertainty and treat them as uncorrelated with each other. This procedure will be applied in Section 4.6.1. Some examples of these categories are:

- $\pi^{+} A \rightarrow \pi^{-} X$ with $x_{F}$ in 0.25-0.5 .

- $\pi^{+} A \rightarrow \pi^{-} X$ with $x_{F}$ in $0.5-0.75$.

- $p A \rightarrow p X$ with $x_{F}$ in 0.75-1.0 . 


\subsubsection{Uncertainty propagation}

We use the so called "multi-universe" technique to propagate uncertainties [60]. This technique is the creation of a statistical ensemble of individual randomly generated universes, where each "universe" selects a value (deviate) for each uncertain parameter. The parameters for the neutrino flux determination are, for example, the hadron production cross-sections. The deviates are drawn from a multi-variable normal distribution centered on the parameter's central values:

$$
N(\vec{x} \mid \vec{\mu} \mathbf{V})=\frac{1}{(2 \pi)^{M / 2} \operatorname{det}(V)^{1 / 2}} \exp \left(-\frac{1}{2}(\vec{x}-\vec{\mu}) \cdot \mathbf{V}^{-1} \cdot(\vec{x}-\vec{\mu})\right)
$$

where $\mathbf{V}$ is the covariance matrix of all $\mathrm{M}$ parameters, $\vec{\mu}$ is the vector of the central values and $\vec{x}$ is the vector of deviates. $\mathbf{V}$ is decomposed by the Cholesky factorization in a lower triangle $\mathbf{L}$ and an upper triangle matrices:

$$
\mathbf{V}=\mathbf{L} \cdot \mathbf{L}^{\mathbf{T}}
$$

Then, the vector of deviates $\boldsymbol{x}$ is given by ${ }^{3}$ :

$$
\vec{x}=\vec{\mu}+\vec{R} \mathbf{L}
$$

where $\vec{R}$ is vector of random Gaussian distributed values with mean zero and unit variance. Every universe predicts a flux spectrum and the resulting spread from the statistical variations is used to evaluate the final flux systematic uncertainty .

Figure 4.10 shows an application of the multi-universe technique for the flux using 50 universes using only the MIPP NuMI pion production weights and assuming $+75 \%$ bin-to-bin correlation for the systematic errors (more on this in Section 4.4.1).

\footnotetext{
${ }^{3}$ The proof of this procedure can be found in the Section 7.4 of $([61])$
} 


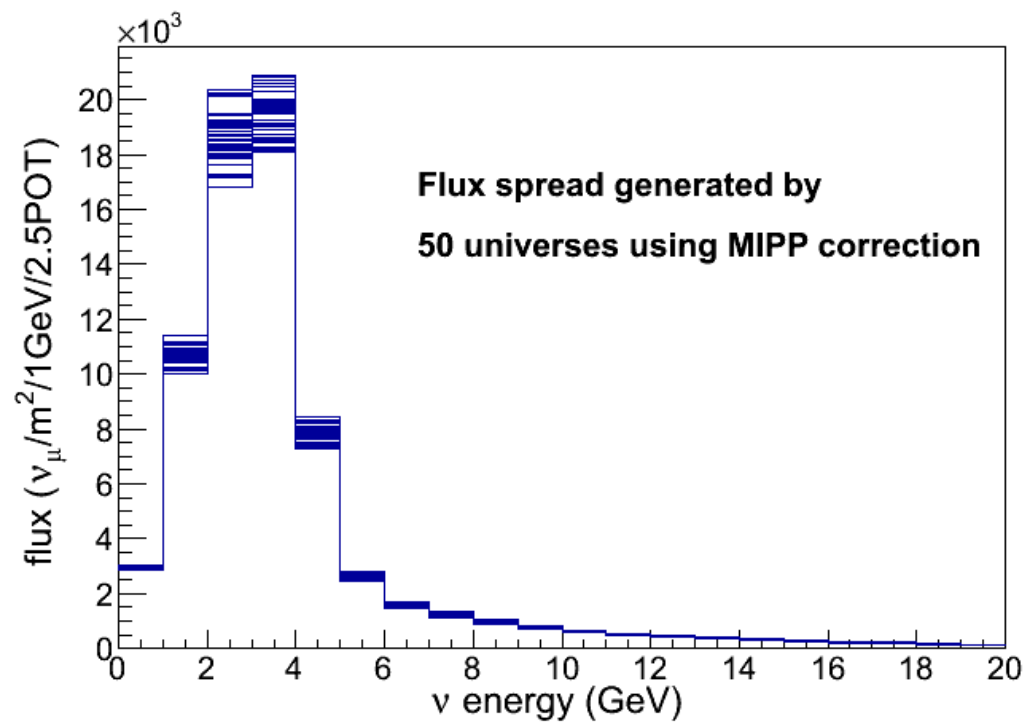

FIG. 4.10: Example of hadron production uncertainty propagation using the multiuniverse technique.

The final correction for a particular universe to the neutrino flux is applied as the product of all corrections given to the neutrino ancestry information.

\subsection{Available data}

We developed our procedure for correcting the simulated flux in the previous section. We now look into the hadronic cascade in NuMI (Section 2.4), to make a survey of all relevant data that can be used in the NuMI beamline.

\section{Thick target data}

These are experiments that collide monochromatic protons on a thick target and measure the yields of hadrons leaving the target. This is the first place to look since they are designed to help the flux determination efforts by using a similar primary beam and the same or replica target as used in the neutrino beamlines. In 
our survey, we found two datasets:

- MIPP: proton on a spare NuMI target at $120 \mathrm{GeV}$ :

- $\pi^{ \pm}$for $p_{Z}$ up to $80 \mathrm{GeV} / \mathrm{c}[62]$.

- $K / \pi$ for $p_{Z}>20 \mathrm{GeV} / \mathrm{c}[63]$.

- NA61: $\pi^{ \pm}$production from proton collisions on a replica T2K target at $31 \mathrm{GeV}$ $[42]^{4}$.

\section{Thin Target Data}

These are experiments that use monochromatic beams on targets of a few percents of interaction lengths. They measure:

- The inelastic and absorption cross-section. Some of the datasets found are:

- Belletini et. al. [64], Denisov et al. [65], etc. : proton, pions and kaons on carbon, aluminum, etc. in a wide energy range.

- NA49 ([66]): proton on carbon at $158 \mathrm{GeV}$.

- NA61 ([40]): proton on carbon at $31 \mathrm{GeV}$.

- Hadron Production. Some of the datasets found are:

- Barton et. al [56]: $p C \rightarrow \pi^{ \pm} X$ at $100 \mathrm{GeV}$ for $x_{F}>0.3$.

- NA49 ([66]): $p C \rightarrow \pi^{ \pm} X$ at $158 \mathrm{GeV}$ for $x_{F}<0.5$.

- NA49 ([67]): $p C \rightarrow n(p) X$ at $158 \mathrm{GeV}$ for $x_{F}<0.95$.

- NA49 ([68]): $p C \rightarrow K^{ \pm} X$ at $158 \mathrm{GeV}$ for $x_{F}<0.2$.

- NA61 ([40]): $p C \rightarrow \pi^{ \pm} X$ at $31 \mathrm{GeV}$.

\footnotetext{
${ }^{4}$ These data were not used in this thesis but it could be used as a cross check in the future.
} 
- MIPP [69]: $\pi / K$ from $p C$ at $120 \mathrm{GeV}$ for $20 \mathrm{GeV} / \mathrm{c}$.

In the next four sections, we explain some details of the experiments and their use in the hadron production correction for the NuMI flux determination, focused on those parts that are relevant to understand their results and uncertainties, as well as the assumptions of our implementation in the computational framework (Section 4.8). Thin target data is split in three sections, one dedicated to the hadron production data (Section 4.5), the second one dedicated to the data extension (Section 4.6) and the other one dedicated to the absorption and inelastic crosssection data (Section 4.7).

\subsection{The thick target datasets}

The thick target data we used come from the experiment MIPP (Main Injector Particle Production) that was dedicated to studying cross-sections and yields of hadron production using thin and thick targets with multiple particle beams at Fermilab. One of its goals was to measure yields of hadrons that exit the NuMI target with low uncertainties in order to help determine the NuMI flux for Fermilab neutrino experiments. 1.43 million events were collected colliding $120 \mathrm{GeV}$ protons from the Main Injector beam on a spare NuMI target. A schematic view of the MIPP detector can be seen in Figure 4.11. More details can be found in [62] and [63].

The basic setup consists of several sub-detectors, each one of which provides particle identification (PID) for a specific momenta range of the particles when they leave the target:

- a Time Projection Chamber (TPC) covering 0.2-1.2 GeV/c.

- a Time of Flight (ToF) system covering 0.5-2.5 GeV/c. 


\section{MIPP \\ Main Injector Particle Production Experiment (FNAL-E907)}

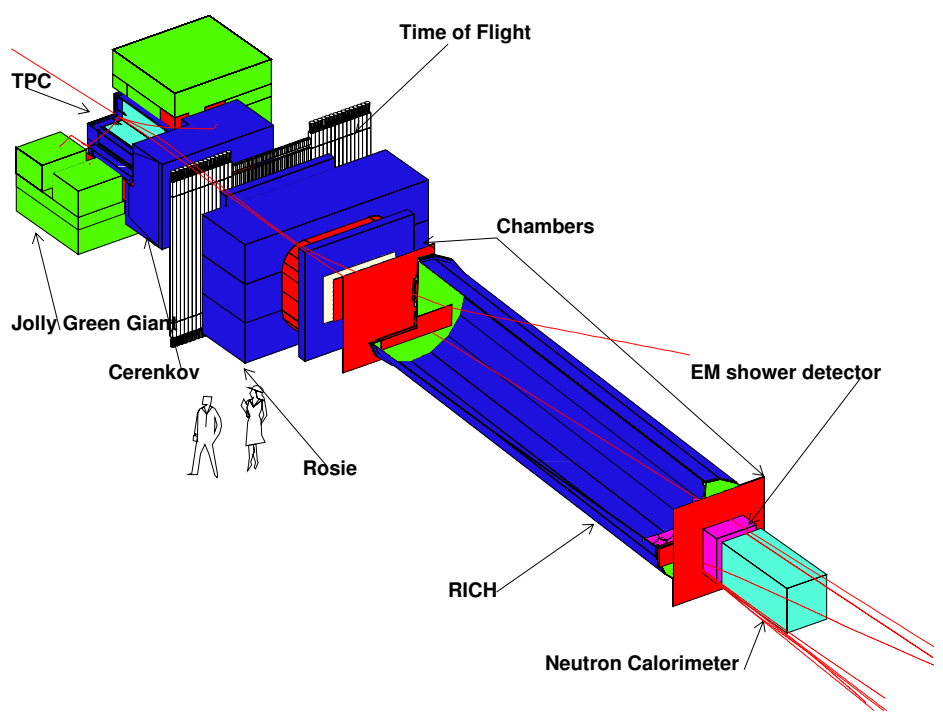

FIG. 4.11: Schematic view of MIPP detector. Taken from [62].

- a segmented gas Cherenkov detector (Ckov) covering 2-20 GeV/c.

- a gas ring imaging Cherenkov detector (RICH) covering 4-80 GeV/c.

For the NuMI target run analysis the particle identification relies on the highly segmented TPC and RICH detectors that give good momentum coverage. The ToF was used to estimate backgrounds in the TPC. The Ckov was not used in this analysis $^{5}$. The low momentum $\left(p_{Z}<2 \mathrm{GeV} / \mathrm{c}\right)$ PID is handled by the TPC while high momentum PID $\left(p_{Z}>20 \mathrm{GeV} / \mathrm{c}\right)$ is handled by the RICH.

To highlight the importance of these data, we quantify the interactions covered by using thick target measurements using our simulation. Figures 4.12 and 4.13 show the average number of inelastic interactions per $\pi^{+}$and per $K^{+}$per MIPP bin, starting from the primary proton beam interaction and counting up all inelastic interactions up to the creation of the hadron that exits the target. They

\footnotetext{
${ }^{5}$ It is complicated for ToF and Ckov to distinguish between particles when there are high multiplicity events.
} 
are separated into three momentum ranges of the particles exited the target: $0-2$ $\mathrm{GeV} / \mathrm{c}, 2-20 \mathrm{GeV} / \mathrm{c}$ and 20-80 GeV/c. The hashed bins represent regions where there are no data due to detector acceptance. For $K^{+}$, only the high momentum is relevant due to the data coverage. See Section 2.4. The minimum possible value is 1 since meson production requires having the generation of new particles from proton-nucleus collisions. Numbers larger than 1 indicate reinteractions in the target. Many of these are incident pions and kaons for which we have no thin target data to use in a correction. See the next section.

There are more than 2 interactions on average for very low momentum pions $(<1 \mathrm{GeV} / \mathrm{c})$ and these values decrease monotonically for higher momentum particles approaching 1.2 in the upper limit of MIPP coverage. Figure 4.12 shows that a significant number of interactions are not covered while the high energy pions and kaons in Figure 4.13 are well covered.

\subsubsection{Thick target pion production}

MIPP NuMI results of $\pi^{+}$and $\pi^{-}$yields [62] are presented in 124 and 119 bins respectively for $p_{Z}$ in $0.3-80 \mathrm{GeV} / \mathrm{c}$ and $p_{T}$ in $0-2 \mathrm{GeV} / \mathrm{c}$ per proton on target (POT). Figure 4.19 shows data published for $\pi^{+}$(left side) and $\pi^{-}$(right side). Different markers represents different $p_{T}$ ranges. Each $p_{T}$ range has been multiplied by a factor to accommodate all the data points in a single plot.

Figures in 4.15 show a combination of the statistical and the background systematic uncertainties for $\pi^{+}$and $\pi^{-}$. Most of the values are small and typically in 2-5\% except for the edges of the sub-detectors, where the errors become bigger. In

our approach, we treat these uncertainties just as statistical, considering them to be uncorrelated between bins.

The additional MIPP systematic uncertainties are: 


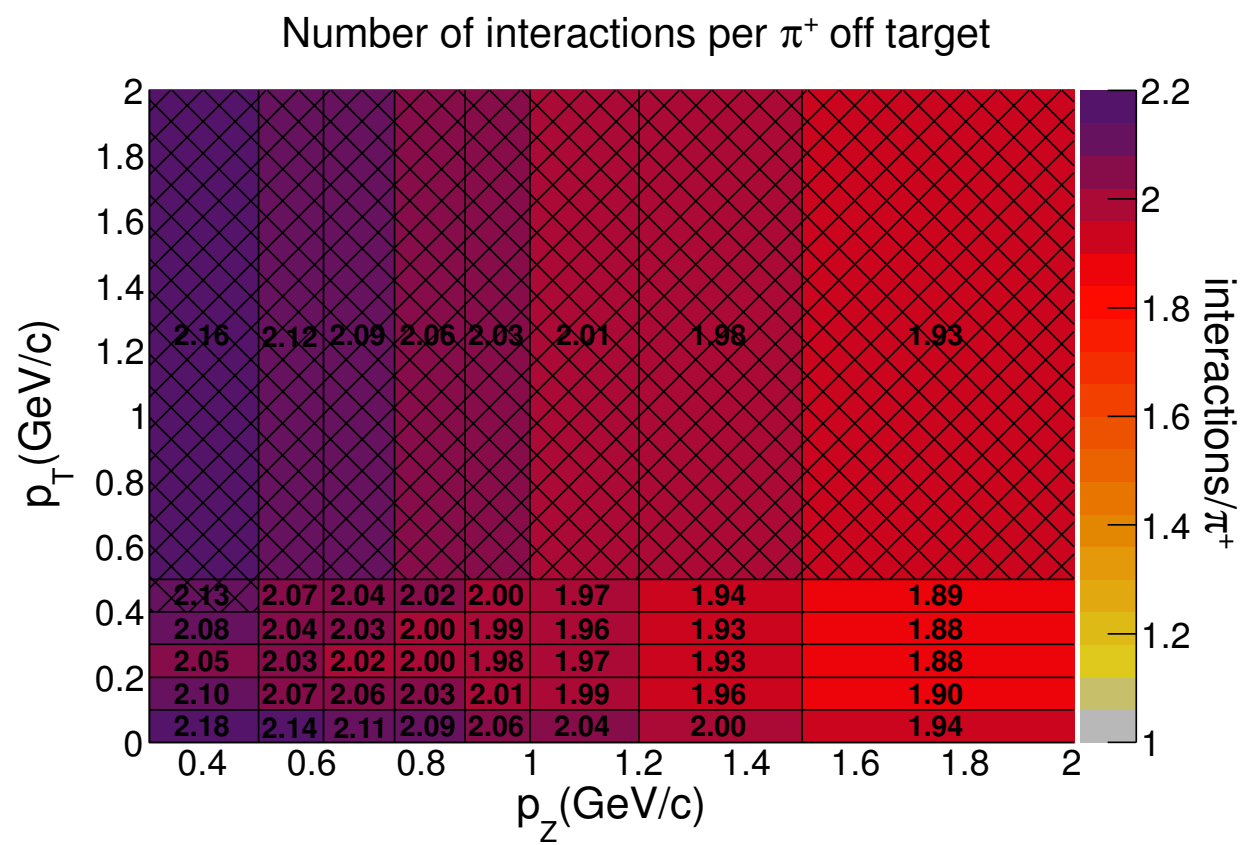

(a)

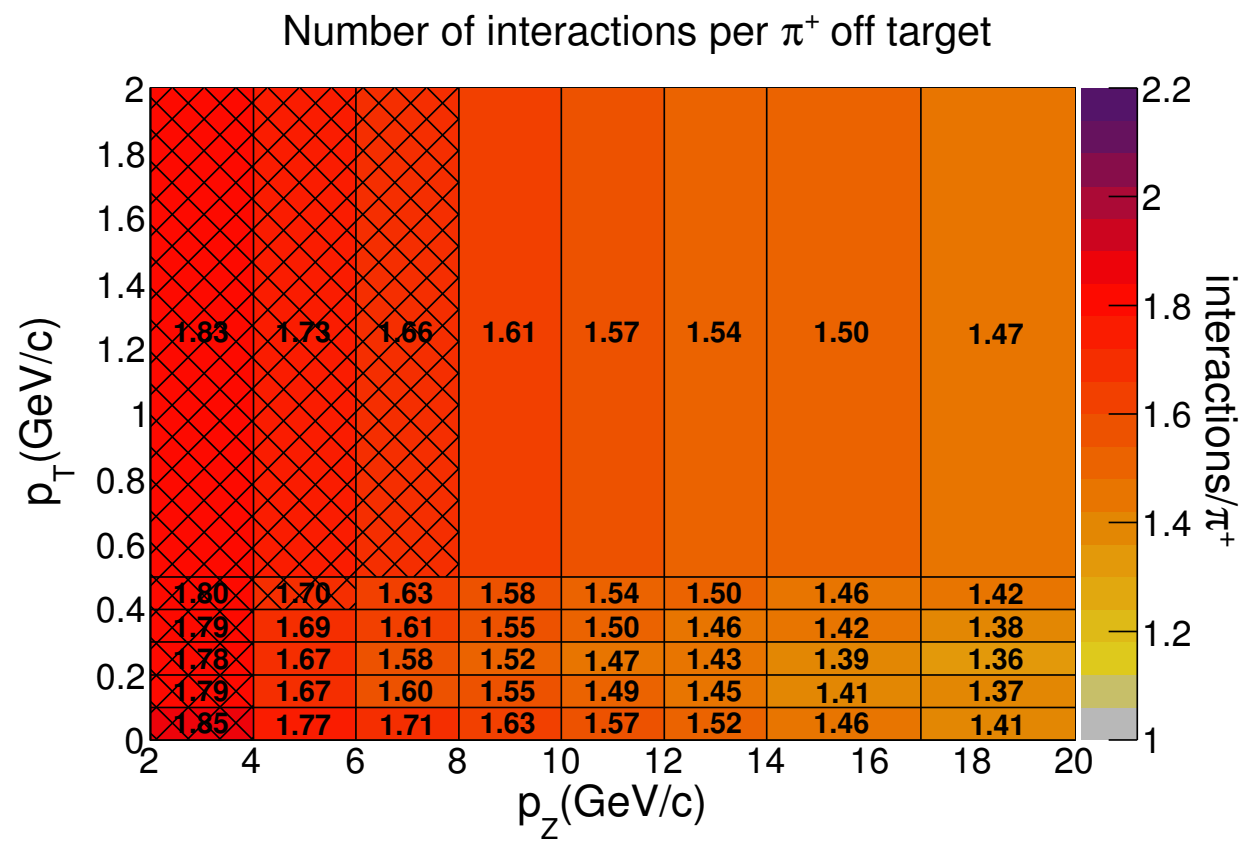

(b)

FIG. 4.12: The importance of secondary interactions: these plots show the average number of inelastic interactions per $\pi^{+}$that exits the target using MIPP binning for $p_{Z}$ in $0-20 \mathrm{GeV} / \mathrm{c}$ according to our NuMI simulation. $p_{Z}$ and $p_{T}$ are the momentum of the $\pi^{+}$when it exited the target. The hashed bins represent regions where there are no data. 


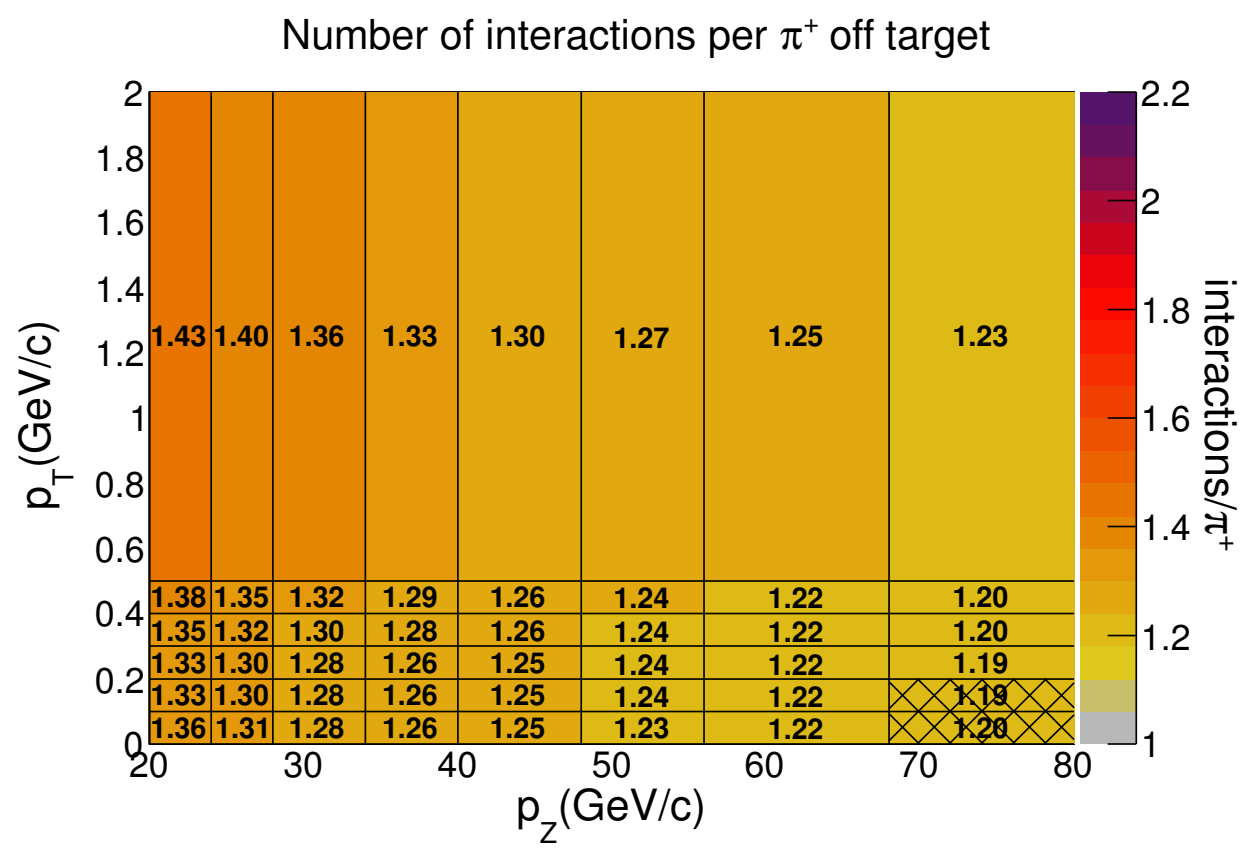

(a) $\pi^{+}$

\section{Number of interactions per $\mathrm{K}^{+}$off target}

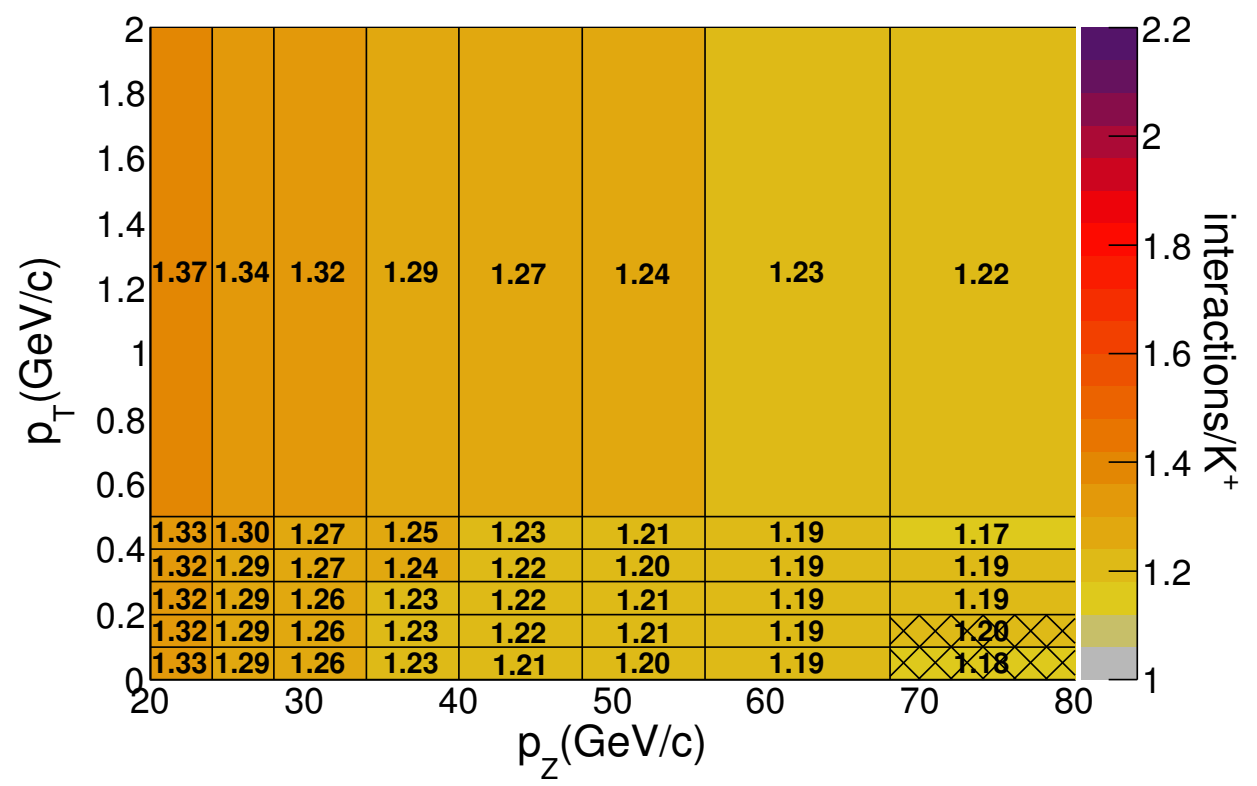

(b) $K^{+}$

FIG. 4.13: The importance of secondary interactions: these plots show the average number of inelastic interactions per $\pi^{+}$and $K^{+}$that exit the target using MIPP binning for $p_{Z}$ in $20-80 \mathrm{GeV} / \mathrm{c}$ according to our flux simulation. $p_{Z}$ and $p_{T}$ are the momentum of the $\pi^{+}$and $K^{+}$when they exited the target. The hashed bins represent regions where there are no data. 

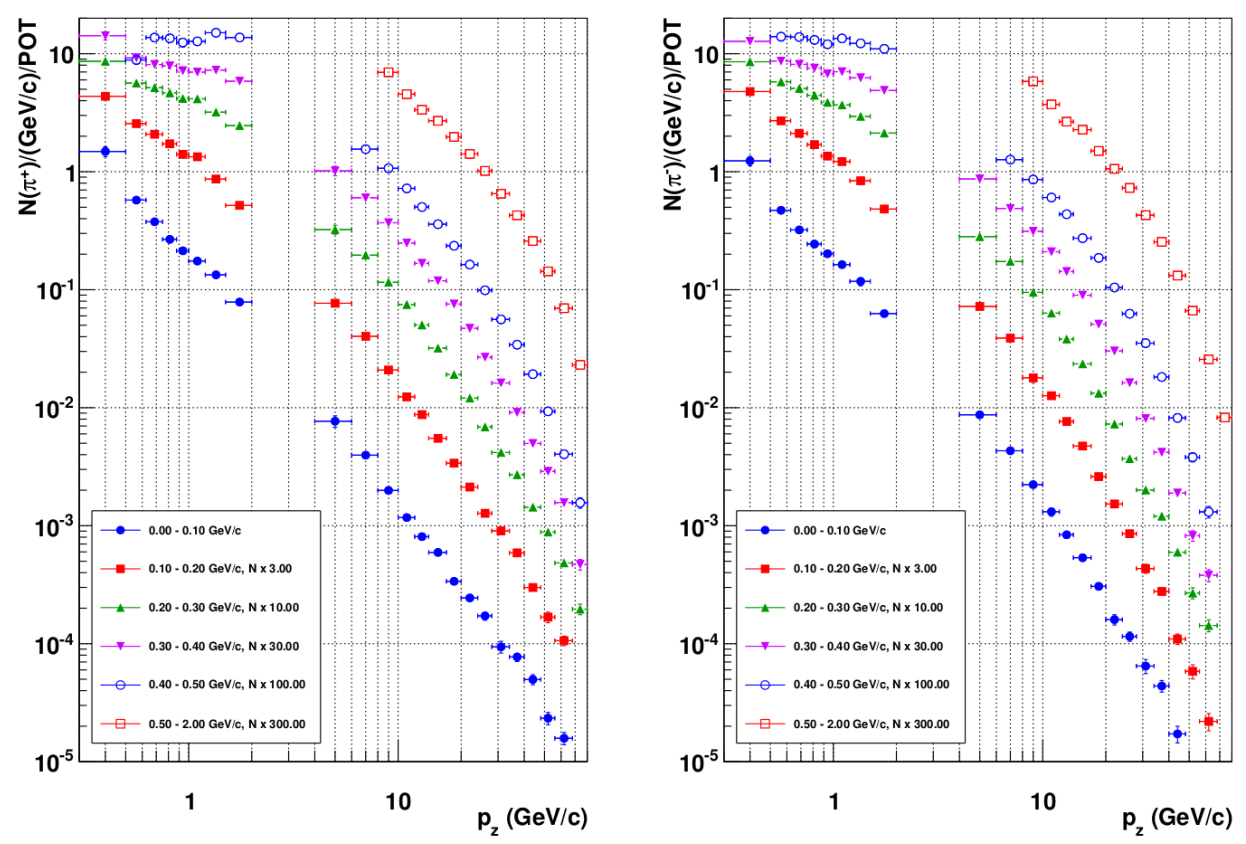

FIG. 4.14: MIPP charged pion production measured at NuMI. The left side is for $\pi^{+}$ and the right side is for $\pi^{-}$. Different markers represents different $p_{T}$ ranges. Every yield has been multiplied by a factor to accommodate them in single plots. Plot taken from $[62]$.

- Momentum scale when a correction is applied to the reconstructed momenta to account for energy loss, scattering and any reconstruction algorithm bias. The uncertainty is $1 \%$ in most of the bins.

- Bin migration due to the momentum resolution and reconstruction failures (4\%) and mismodeling of the noise (1\%).

- Detector Modeling in the MC (2\%).

- Pileup due to an improper modeling of overlapping tracks, specially for high multiplicity events. This is the dominant uncertainty, typically a few percent but it can be up to $10 \%$ for some bins.

The figures in 4.16 show these additional systematics errors added in quadrature for $\pi^{+}$and $\pi^{-}$. Nearly all bins are in the 4-5\% range but large bin-to-bin correlations within each sub-detector are expected. 


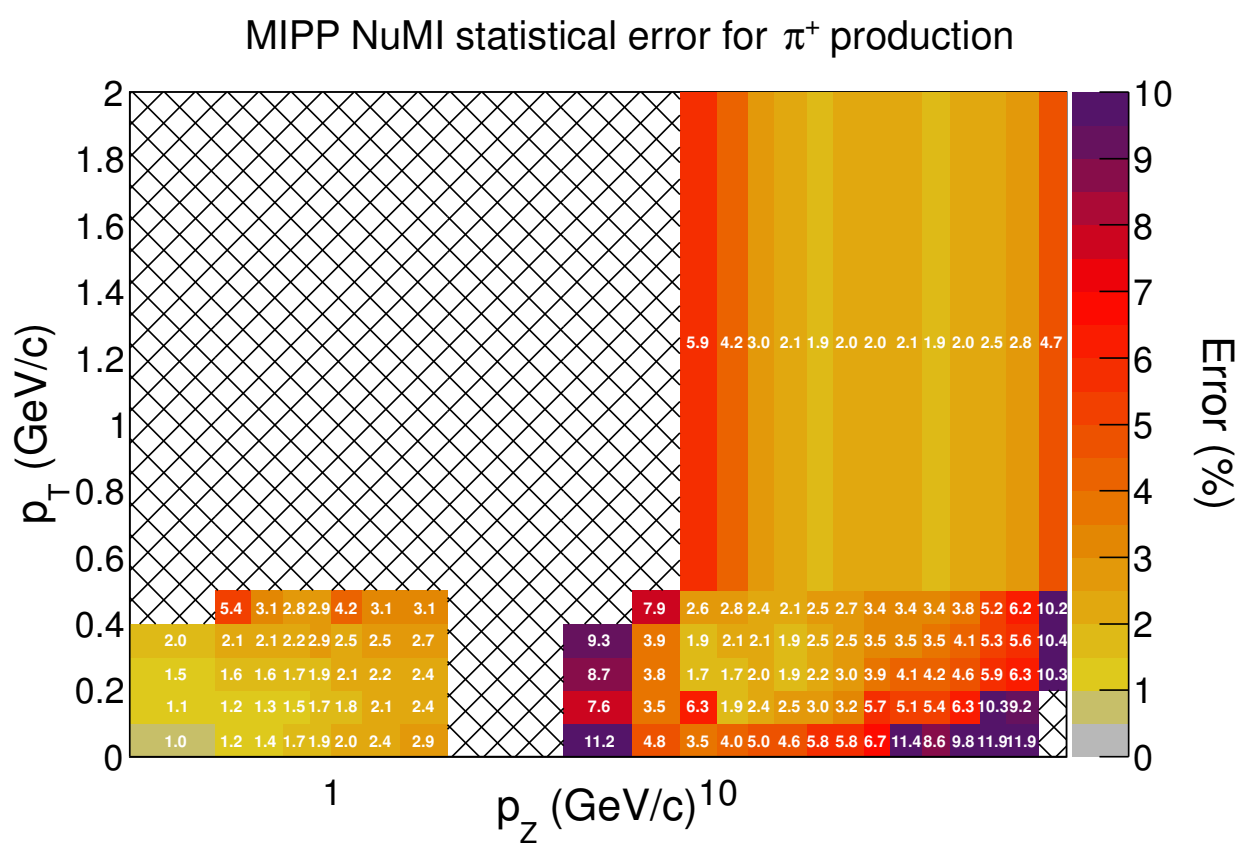

(a) $\pi^{+}$.

MIPP NuMI statistical error for $\pi$ - production

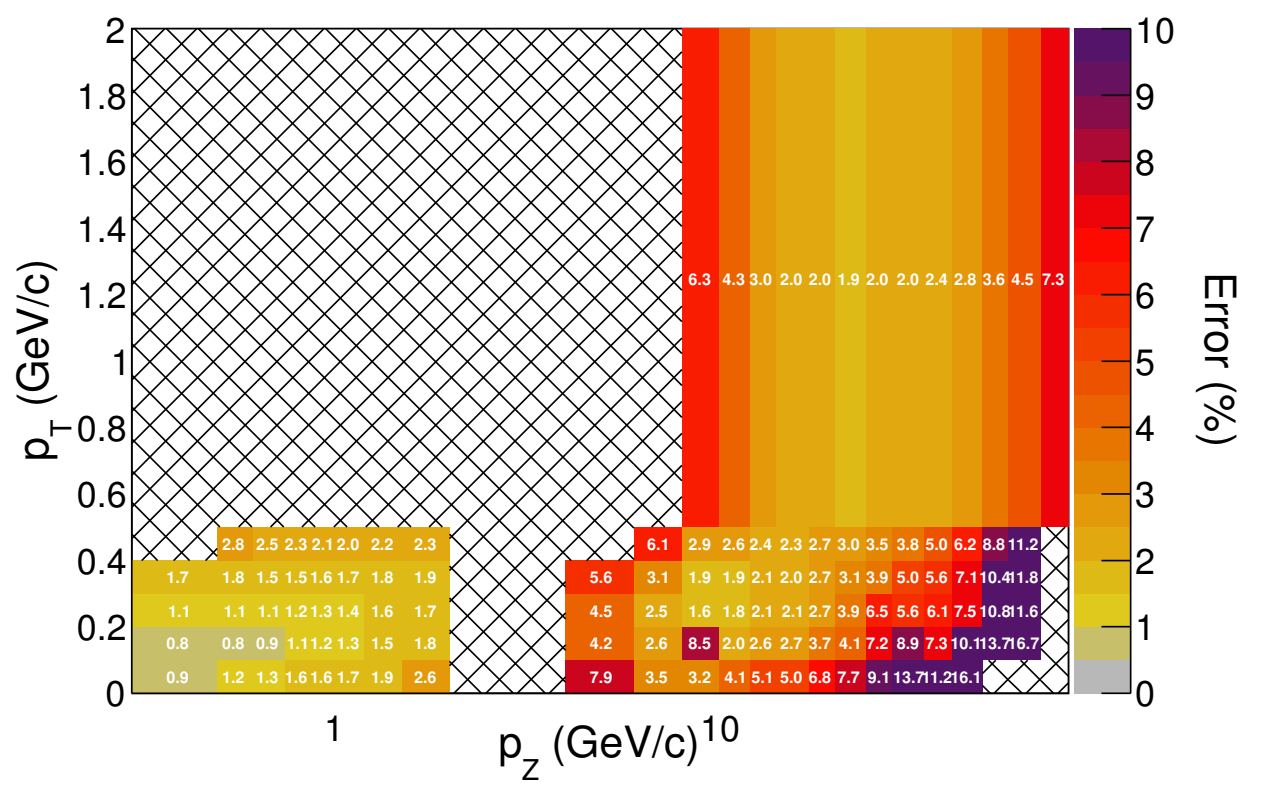

(b) $\pi^{-}$.

FIG. 4.15: MIPP NuMI charged pion statistical uncertainties. Numbers in every bins indicate the exact uncertainty value. The hashed bins represent regions where there are no data. 


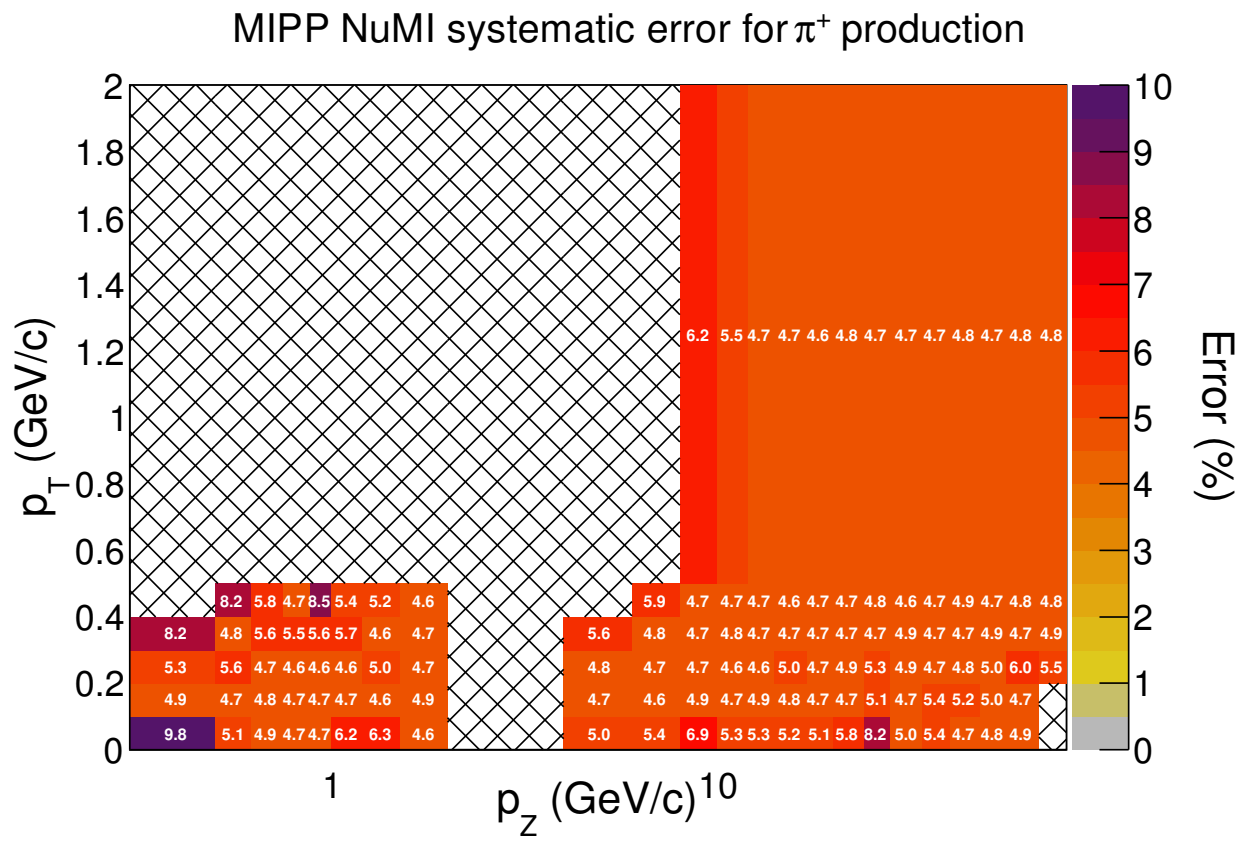

(a) $\pi^{+}$.

MIPP NuMI systematic error for $\pi^{-}$production

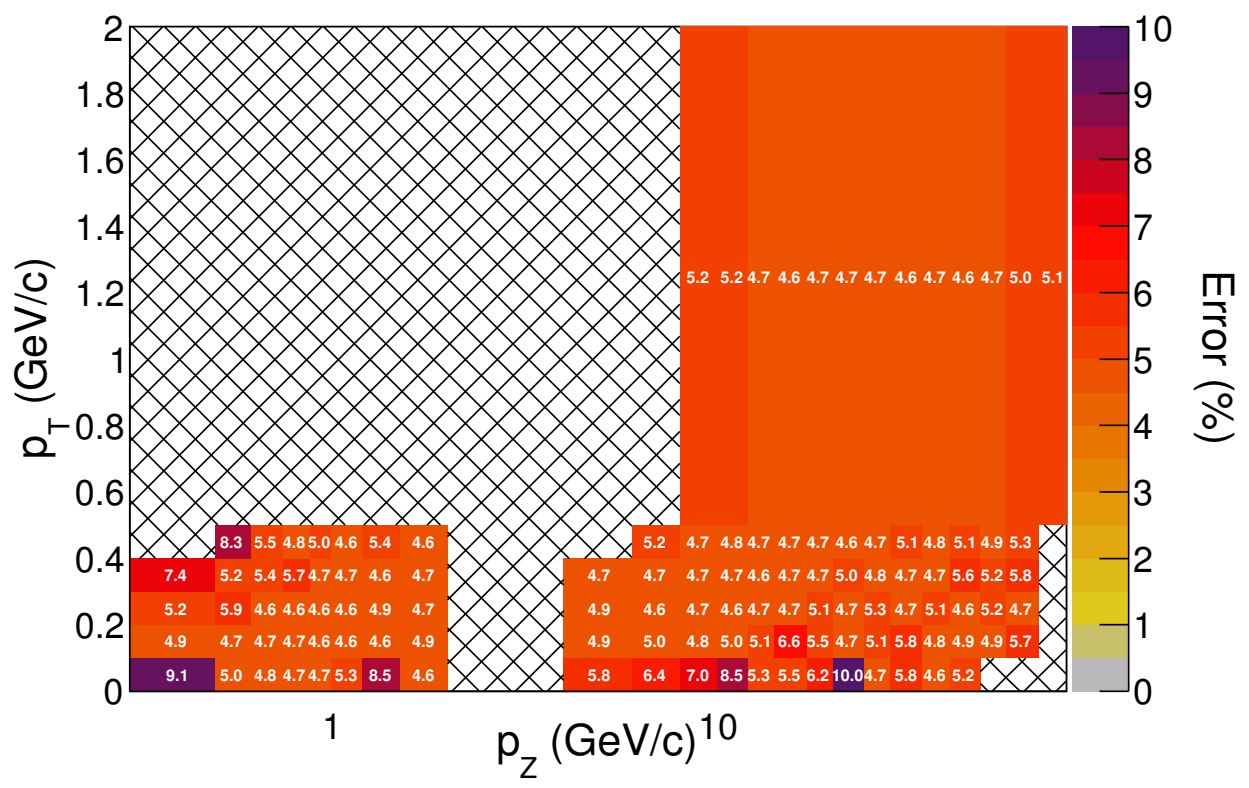

(b) $\pi^{-}$.

FIG. 4.16: MIPP NuMI charged pion systematic uncertainties. Numbers in every bins indicate the exact uncertainty value. The hashed bins represent regions where there are no data. 
The effects of using four different positive bin-to-bin correlation assumptions of their systematic errors are shown in Figure 4.17. The fractional errors on the flux correspond to the part of the $\nu_{\mu}$ flux that is affected by MIPP (i.e., the neutrinos that have one pion correctable by MIPP). As we expect, when we increase the correlation, the fractional error increases accordingly. For example, the values go from $\sim 2 \%$ for no correlation scenario to $\sim 5 \%$ for a totally correlated case around $8 \mathrm{GeV}$ neutrino energy.

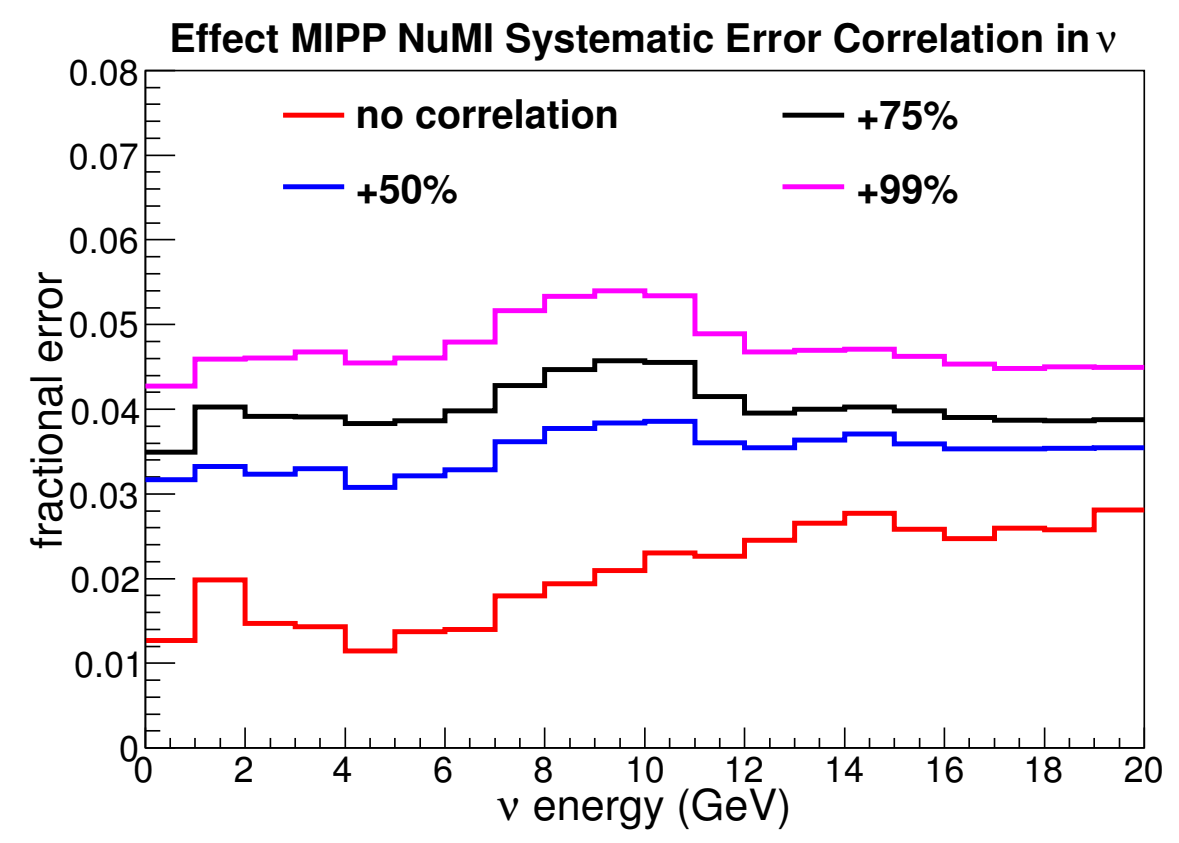

FIG. 4.17: Effect of the bin-to-bin correlation of the systematic uncertainties. The fractional error comes from systematic uncertainties applied to the $\nu_{\mu}$ flux affected by MIPP assuming 4 possible correlations: none, $+50 \%,+75 \%$ and $+99 \%$.

\section{Technical details of the implementation}

MIPP NuMI charged pion correction uses Equation 4.11. The implementation is as follows :

1. As was mentioned above, we treat the statistical + background systematic uncertainties as just statistical. In that way, they enter as uncorrelated to the 
additional systematics presented.

2. A positive $75 \%$ correlation is used between bins for the systematic uncertainties within TPC and RICH. Bins from different sub-detectors are uncorrelated.

3. We have not made any data interpolation and the correction is applied directly when the kinematics of a pion falls on a bin edge.

4. To calculate the factor $y_{D a t a}$ in Equation 4.11, we look at the percentage of primary protons that pass through and leave the replica target without interacting in MIPP NuMI which is $13.5 \pm 2-3 \%$. This is based on the number of reconstructed tracks per proton incident on the target and assumes that one-track events are just primary protons ${ }^{6}$. This uncertainty comes from the reconstruction efficiency. We use $3 \%$ for this uncertainty.

5. In the MC, we found that $13.28 \%$ of primary protons leave the target without interacting or after a quasi-elastic interaction and then we use this value to calculate $y_{M C}$.

Figures in 4.18 show the MIPP charged pion weights using the Equation 4.11. The Z-axes are restricted to $[0.5,2.0]$ since all weights are inside this range. For low momentum, the MC underestimates most of $\pi^{+}$yields for TPC bins. For RICH bins, there are two clear regions, one of them where MC overestimates the yields $\left(10 \mathrm{GeV} / \mathrm{c}<p_{Z}<30 \mathrm{GeV} / \mathrm{c}\right.$ and $\left.p_{T}<0.4 \mathrm{GeV} / \mathrm{c}\right)$ up to $25 \%$, and another one where the $\pi^{+}$are overestimated for $\sim 50 \%$.

For $\pi^{-}$weights, the TPC bins have similar patterns as $\pi^{+}$, however, for RICH, they are dissimilar. Apart for $20 \mathrm{GeV} / \mathrm{c}<p_{Z}<50 \mathrm{GeV} / \mathrm{c}$ and $p_{T}<0.4 \mathrm{GeV} / \mathrm{c}$ where the MC overestimates $\pi^{-}$, the rest of the bins have small corrections. These will cause different effects in the $\nu_{\mu}$ than $\bar{\nu}_{\mu}$ fluxes.

${ }^{6}$ These values were established after an email exchange with J. Paley (main author of [62]). 


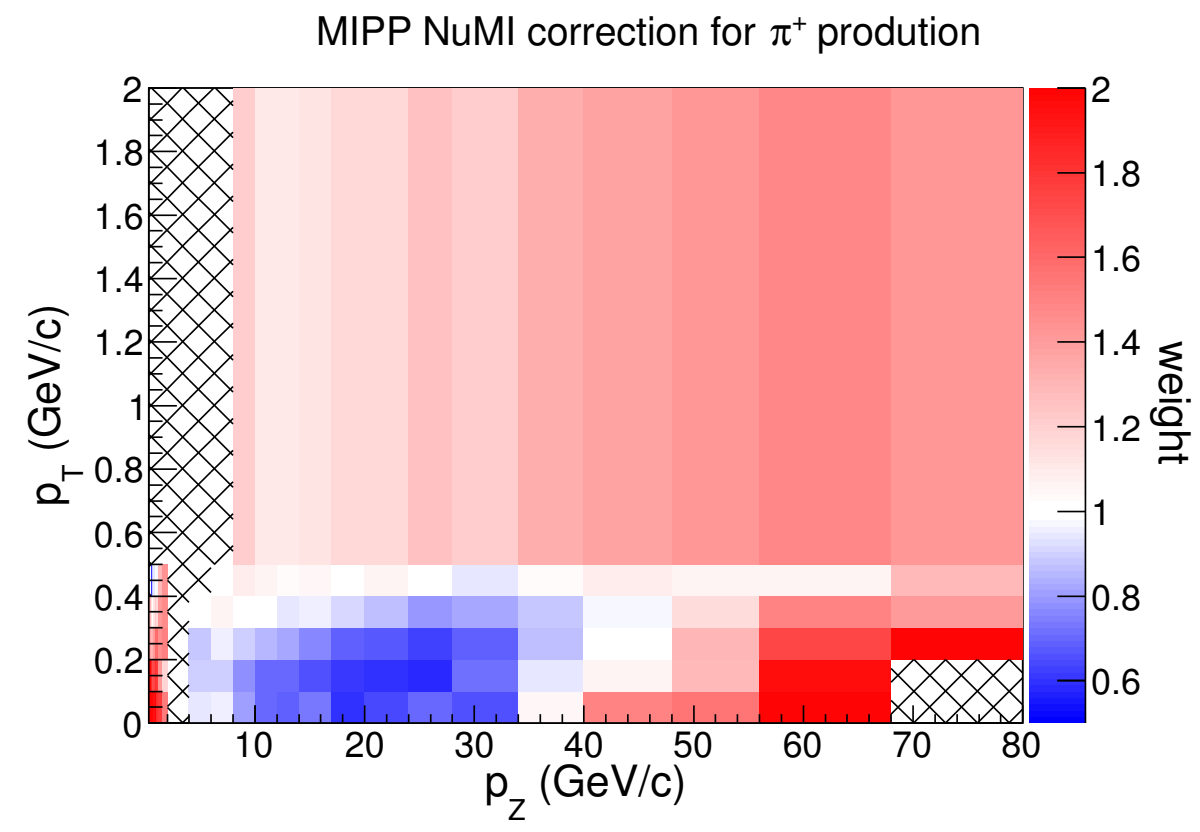

(a) $\pi^{+}$.

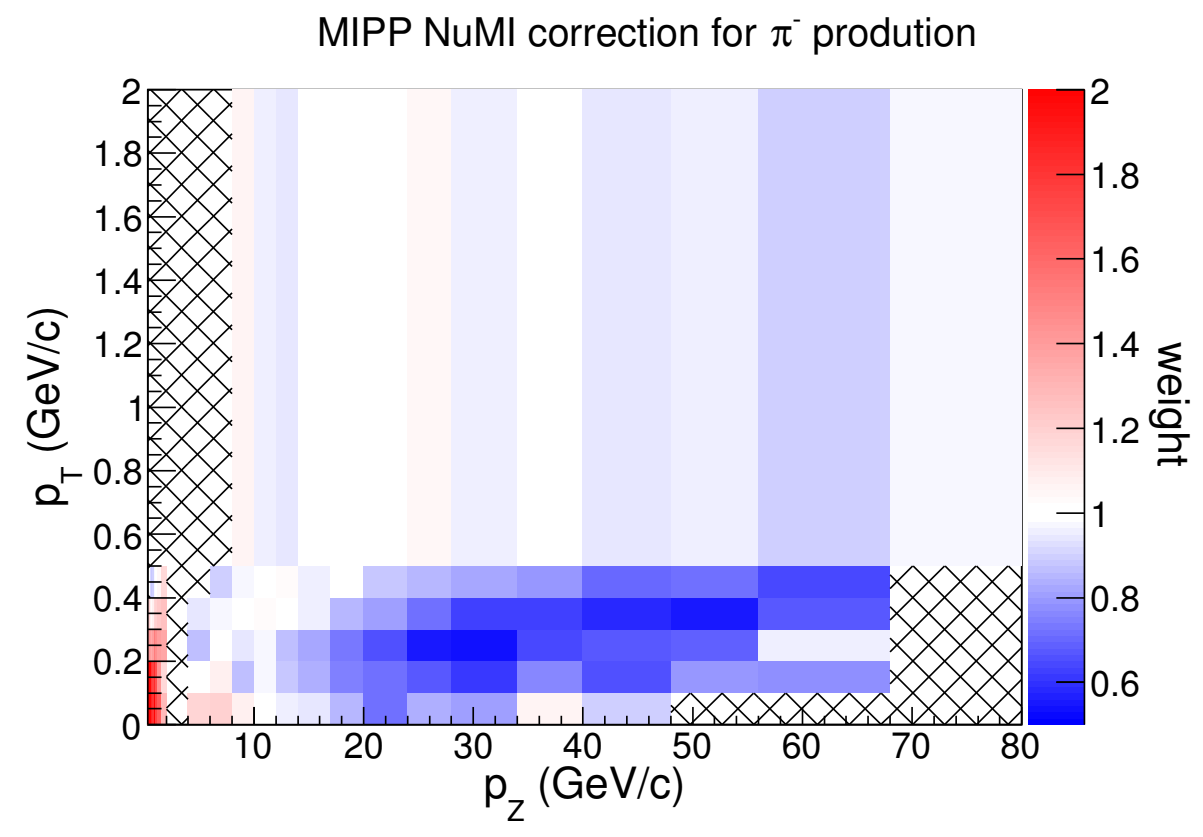

(b) $\pi^{-}$.

FIG. 4.18: MIPP NuMI charged pions weights. 


\subsubsection{Thick target kaon production}

We extract kaon production by combining pion yields from [62] and the $K / \pi$ ratios reported in [63] for longitudinal momentum $\left(p_{Z}>20 \mathrm{GeV} / \mathrm{c}\right)$. For the ratio analysis, only the RICH was used and the results are presented in 24 bins.
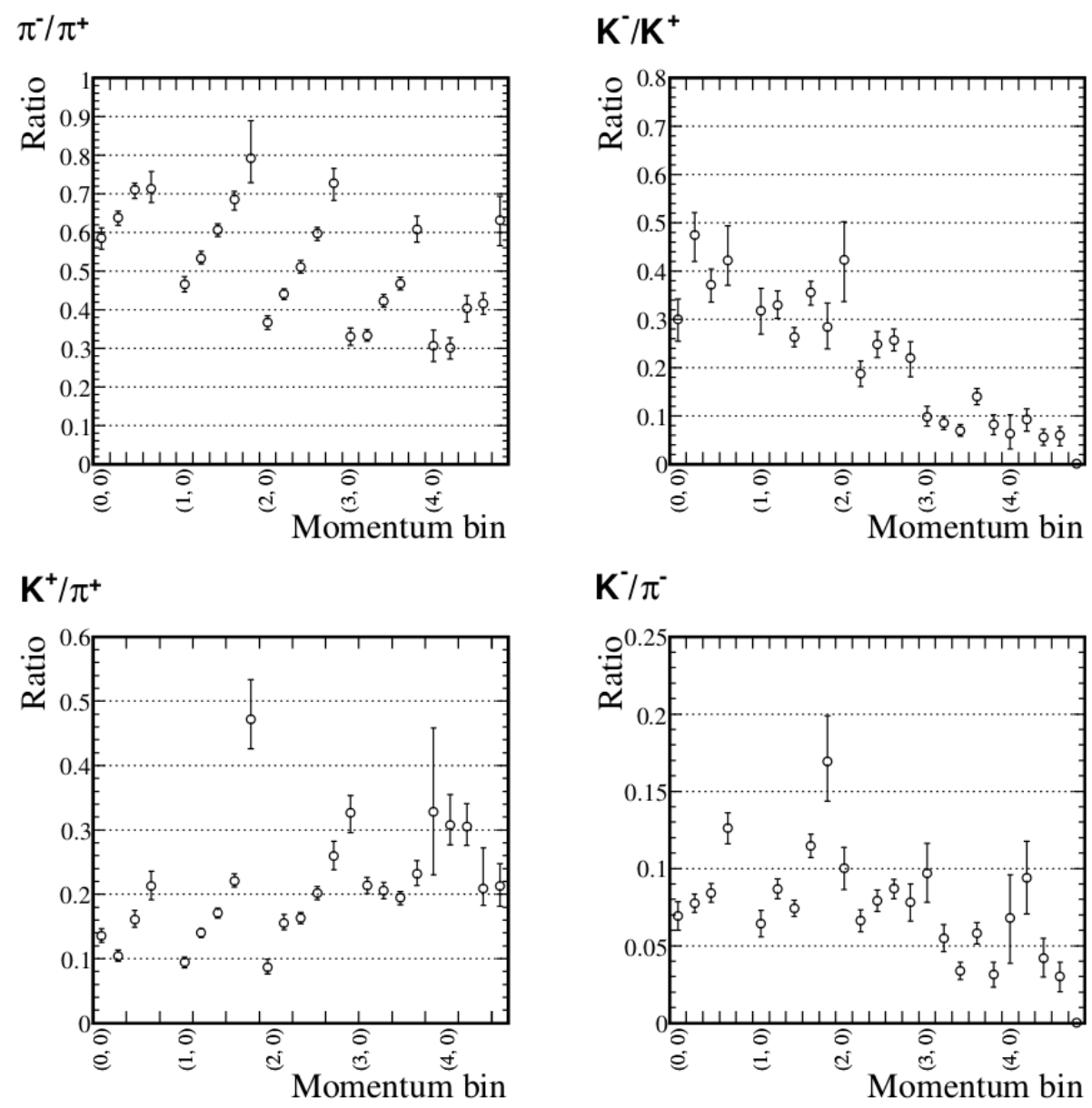

FIG. 4.19: $\operatorname{MIPP} \pi^{-} / \pi^{+}, K^{-} / K^{+}, K^{+} / \pi^{+}$and $K^{-} / \pi^{-}$ratios. The momentum bin convention $(\mathrm{X}, \mathrm{Y})$ is such that $\mathrm{X}$ represent the longitudinal momentum and $\mathrm{Y}$ the transverse momentum. $\mathrm{X}(\mathrm{GeV} / \mathrm{c})=0(20-24), 1(24-31), 2(31-42), 3(42-60), 4(60-90) . \mathrm{Y}(\mathrm{GeV} / \mathrm{c})$ $=0(<0.2), 1(0.2-0.4), 2(0.4-0.6), 3(0.6-1.0), 4(>1.0)$. Plot taken from [63].

Figure 4.20 shows the statistical errors of $K^{+} / \pi^{+}$and $K^{-} / \pi^{-}$and as can be noted, the values are high, especially in the latter case.

The systematic uncertainties on $\pi / K$ were evaluated independently from the pion yield analysis presented above and we do not have enough information to cancel 


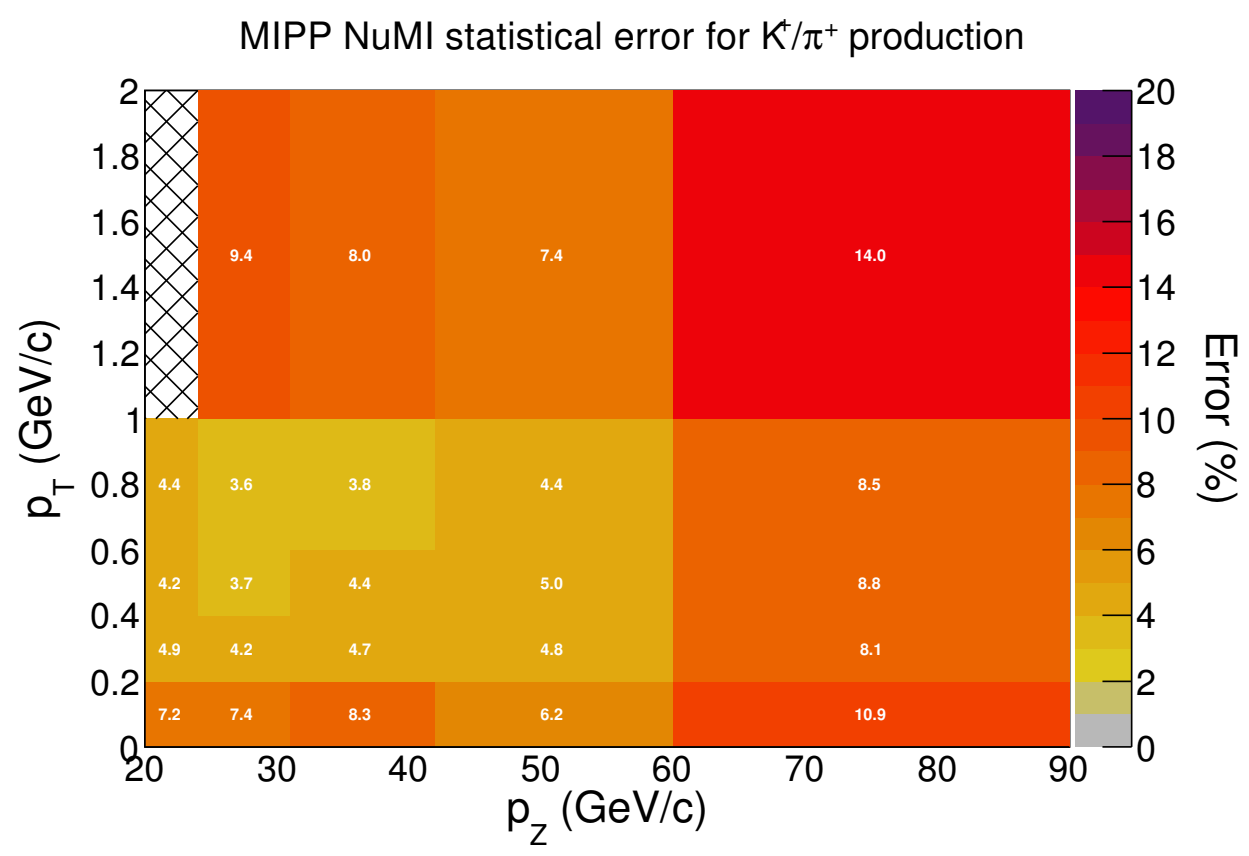

(a) $K^{+} / \pi^{+}$

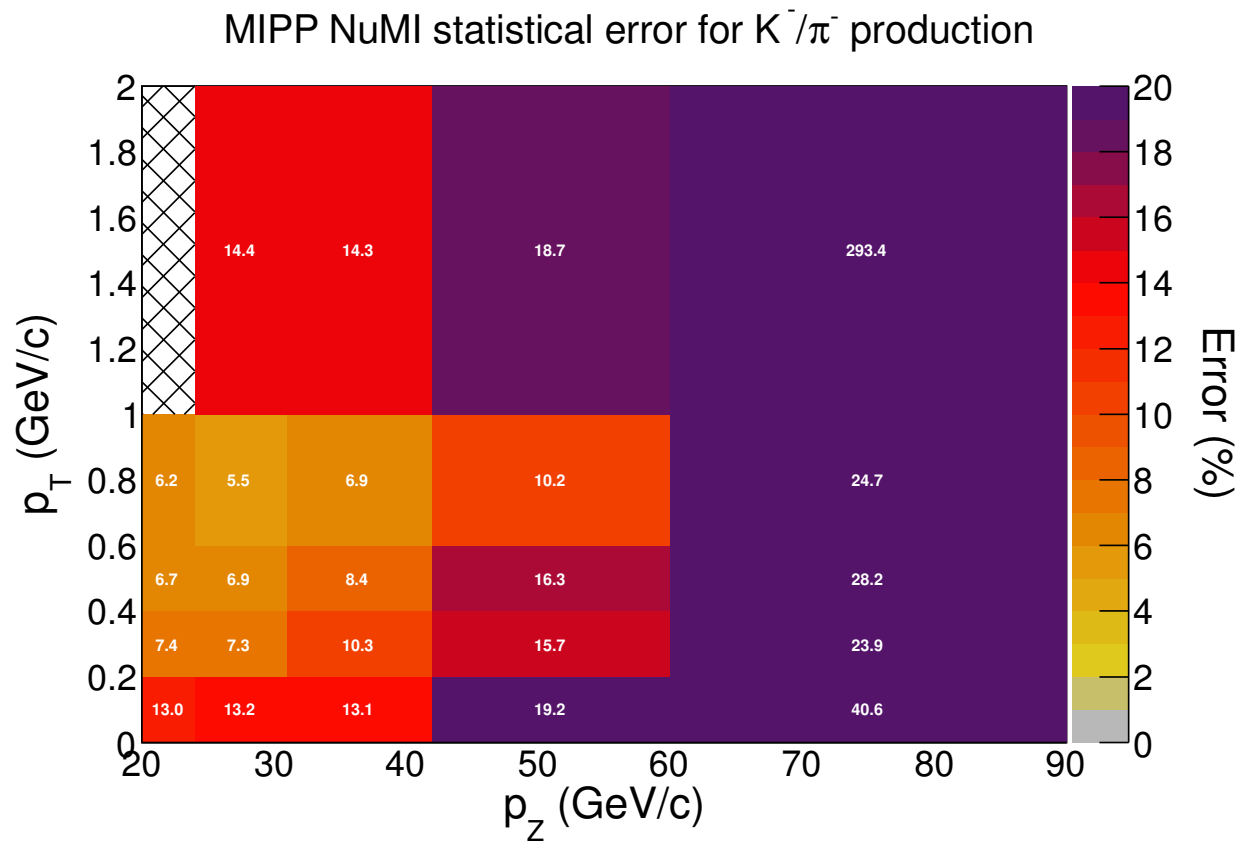

(b) $K^{-} / \pi^{-}$.

FIG. 4.20: MIPP NuMI statistical uncertainties for $K / \pi$. Numbers in every bins indicate the exact uncertainty value. The hashed bins represent regions where there are no data. 
any correlations between them. The main sources on the systematic uncertainties on the ratio are:

- Beam: estimating the effect of small changes of the proton beam profile on the ratio $(<4 \%)$.

- Momentum: accounting for the differences between the measured momentum in data with respect to $\mathrm{MC}$. The effect on the ratio is $\pm 5 \%$ for most of the bins.

- Background subtraction, typically less than $20 \%$.

Figures in 4.21 show the systematic errors added in quadrature for $K^{+} / \pi^{+}$and $K^{-} / \pi^{-}$. The values are large in comparison to pions, especially for high longitudinal momentum.

\section{Technical details of the implementation}

The implementation of the MIPP NuMI kaon correction is as follows:

1. The $K / \pi$ statistical and systematic uncertainties are considered as uncorrelated. No bin-to-bin correlation assumption is made for the systematic uncertainties on the ratio.

2. No interpolation is applied to the data.

3. The $K^{ \pm}$yields are determined by multiplying the $\pi^{ \pm}$yields and $K^{ \pm} / \pi^{ \pm}$ratios in each universe.

4. A $K_{S(L)}^{0}$ correction is calculated by using the Equation 4.21 .

Figures in 4.22 and 4.23 show the MIPP charged and neutral kaon weights using formula 4.11 after the kaon yield determination. The Z-axes are restricted to $[0.5,3.0]$ but $K^{+}$weights can be higher for $p_{Z}>56 \mathrm{GeV} /$ c. Except for $p_{Z}<$ 


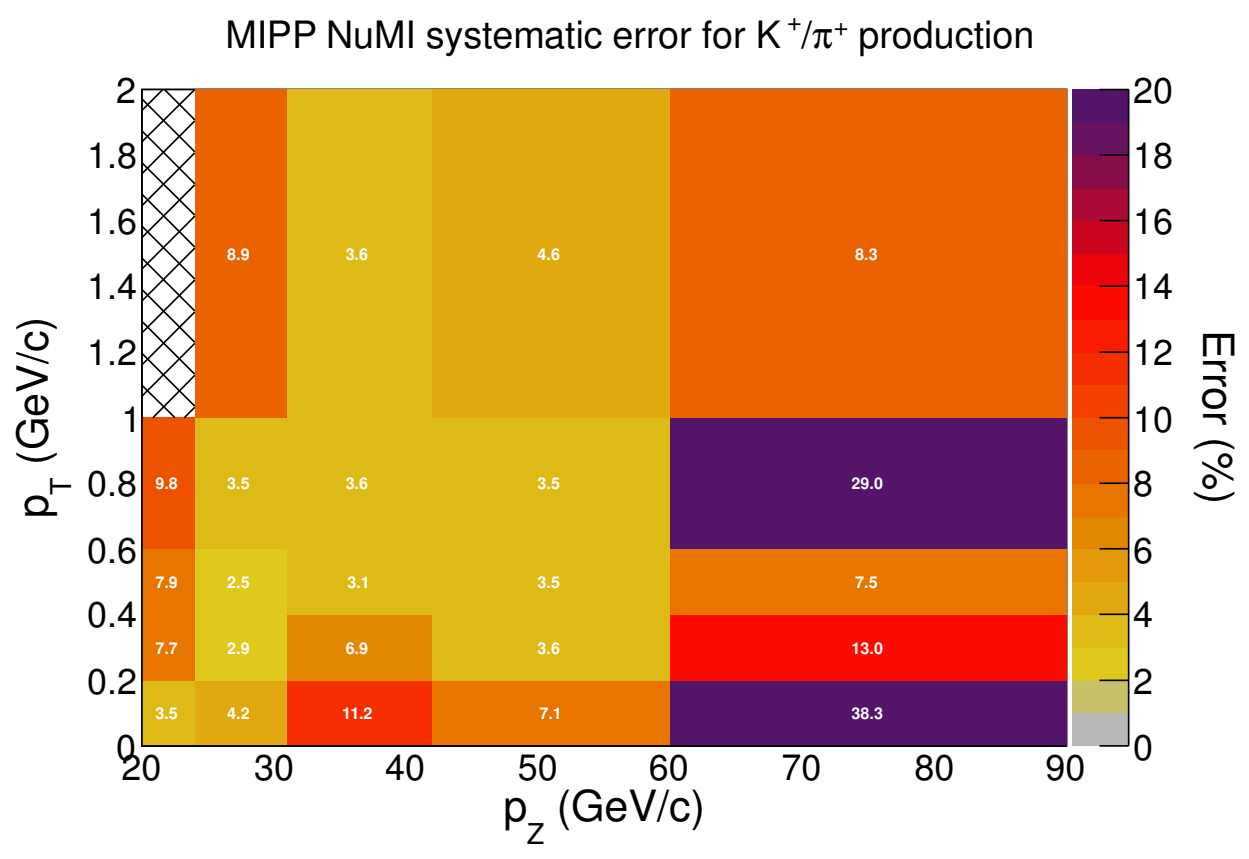

(a) $K^{+} / \pi^{+}$.

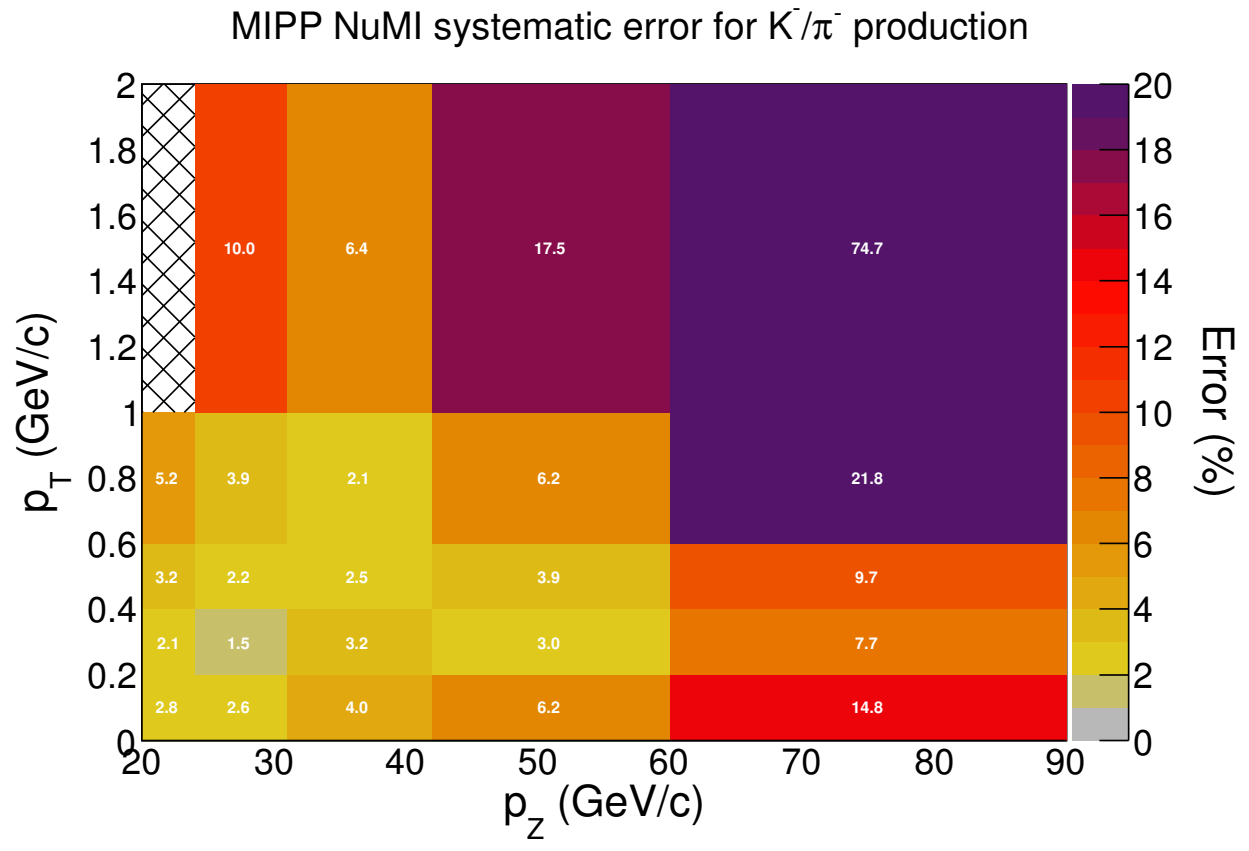

(b) $K^{-} / \pi^{-}$.

FIG. 4.21: MIPP NuMI statistical systematic for $K / \pi$. Numbers in every bins indicate the exact uncertainty value. The hashed bins represent regions where there are no data. 
$40 \mathrm{GeV} / \mathrm{c}$ and $p_{T}<0.4 \mathrm{GeV} / \mathrm{c}$ the $\mathrm{MC}$ underestimates the $K^{+}$yields. $K^{-}$and neutral weights have, in general, smaller values than $K^{+}$. 


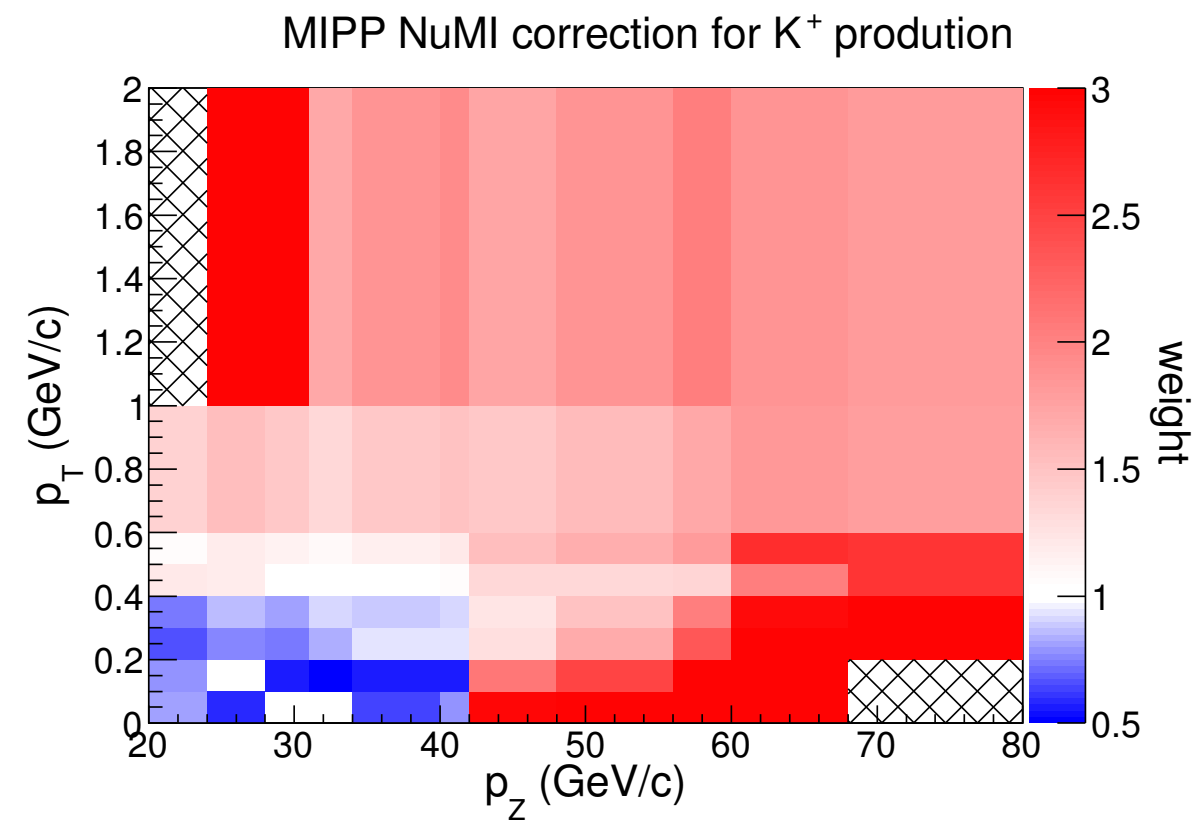

(a) $K^{+}$.

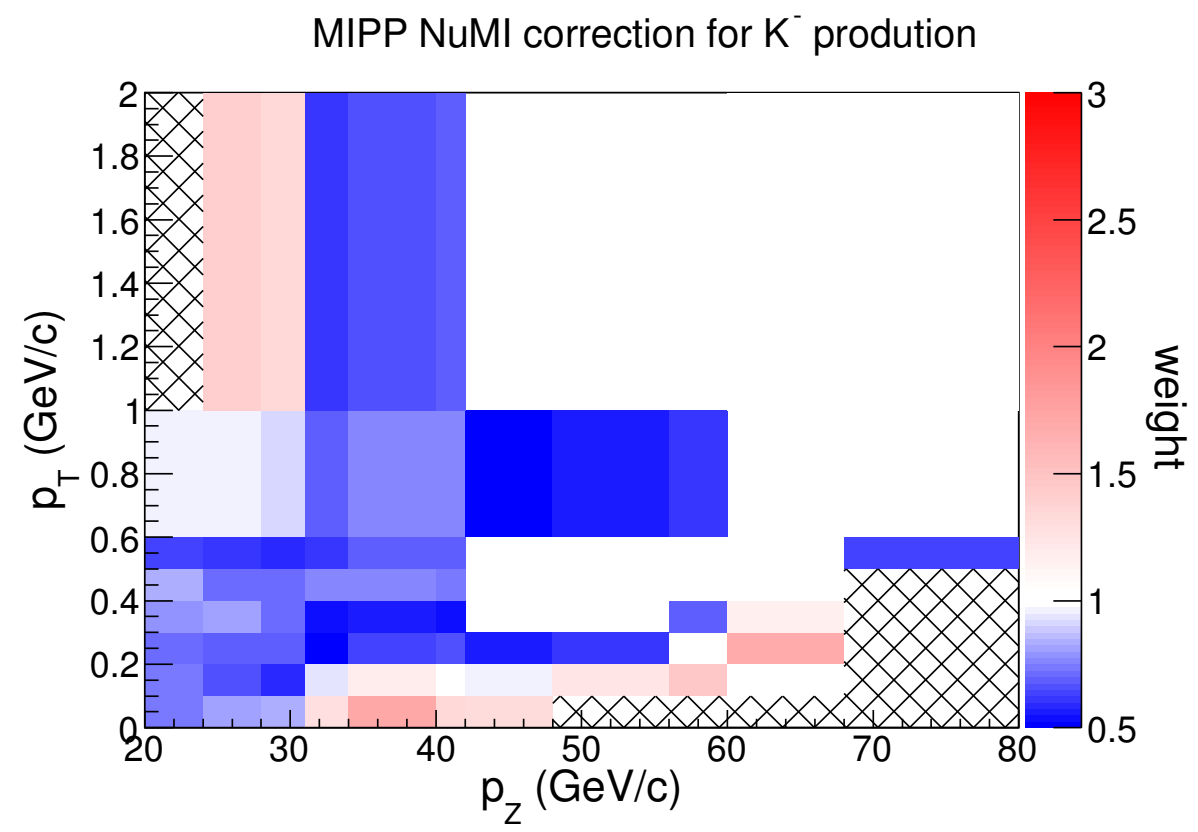

(b) $K^{-}$.

FIG. 4.22: MIPP NuMI charged kaons weights. 


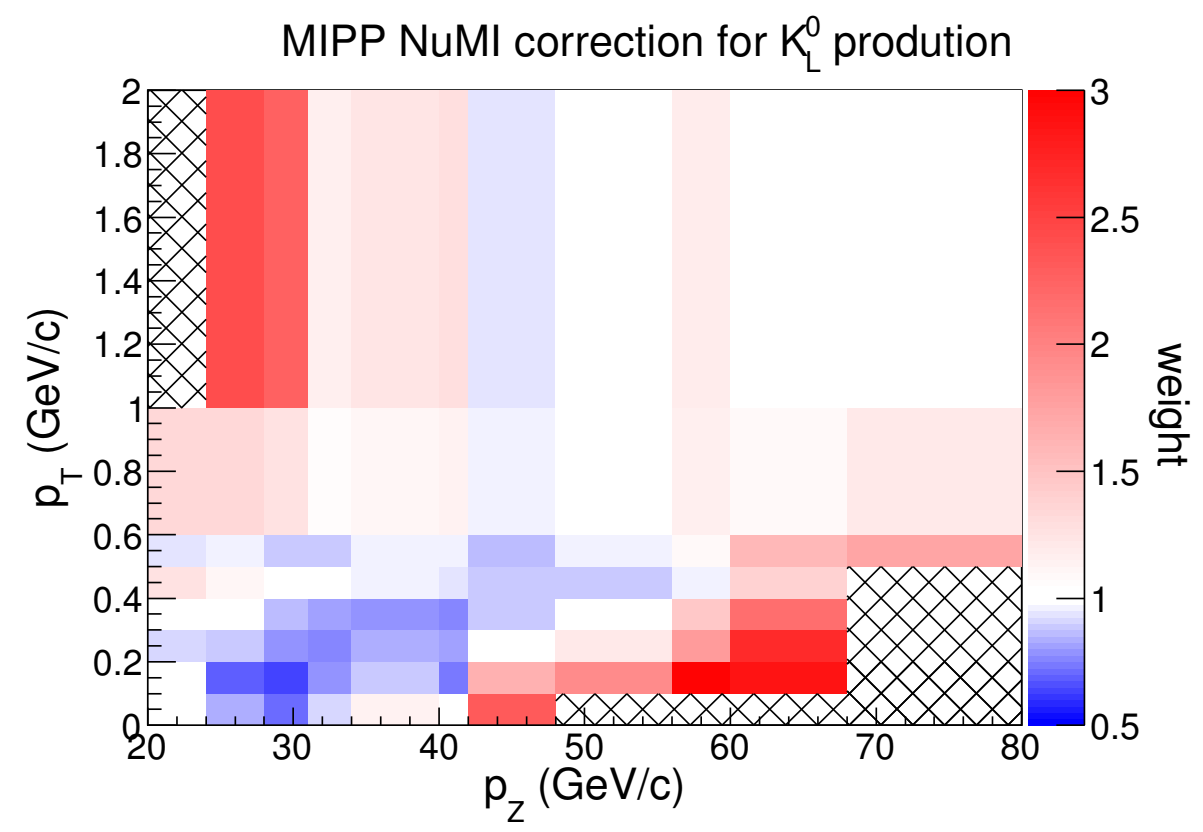

(a) $K_{L}^{0}$.

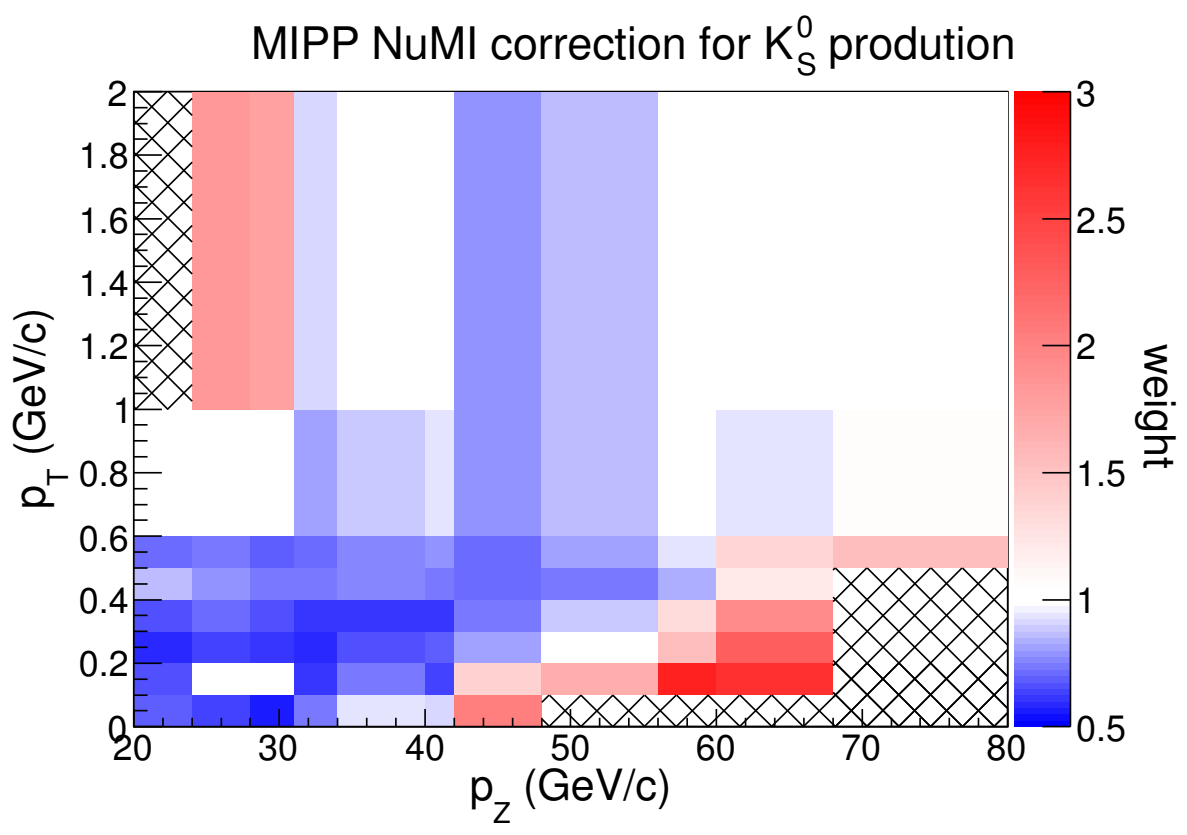

(b) $K_{S}^{0}$.

FIG. 4.23: MIPP NuMI neutral kaons weights. 


\subsection{The thin target hadron production datasets}

The thin target hadron production datasets we used essentially come from NA49. Barton and MIPP are used in conjunction with NA49 to extend its data coverage.

\subsubsection{NA49}

The NA49 experiment's goal is to make a comprehensive study of soft hadronic interactions using different beams and thin targets. Their relevant results for NuMI are the measurements of hadron production in proton-carbon interactions at $158 \mathrm{GeV} / \mathrm{c}$ beam momentum. 377,000 inelastic events in the H2 beam line at CERN SPS accelerator complex were recorded from a beam composed by $65 \%$ protons, $30 \%$ pions and $5 \%$ kaons. Charged pions and protons invariant differential cross-section as well as neutron yields were published in a wide $x_{F}$ coverage $[66,67]$. There are also low $x_{F}$ charged kaon data from a MINOS thesis [68].

A schematic view of the NA49 detector can be seen in Figure 4.24. More details can be found in [70].

The basic setup consists of four large Time Projection Chambers (TPC):

- Two Vertex TPC's just downstream of the target, together with two aperture Vertex Magnets, are used for interaction vertex, particle tracking and PID determination.

- Two Main TPC's are located downstream of the magnets in order to extend the acceptance to higher momentum and provide sufficient track length for PID.

The NA49 interaction trigger system consisted of a small scintillation counter ( $2 \mathrm{~cm}$ in diameter) located $380 \mathrm{~cm}$ from the target and between the vertex TPC's (Figure 4.24). This operated in anti-coincidence with the primary beam. 


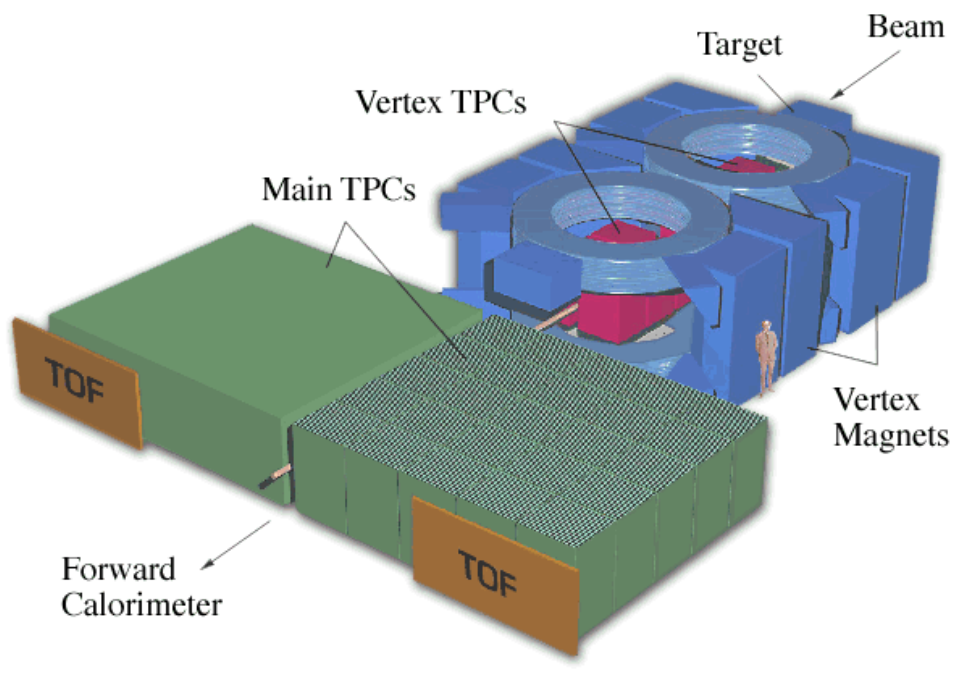

FIG. 4.24: Schematic view of NA49 detector. Taken from [71].

The main systematics in NA49 come from:

- Normalization in determining the inelastic cross-sections from the interaction trigger. A trigger scintillator counter was placed in anti-coincidence with the beam. This systematic accounts for any losses of protons, pions and kaons when they hit the trigger as well as subtracting the elastic component. The uncertainty is $2.5 \%$ and it is directly applicable to the differential cross-sections. For neutron yield it is $1.5 \%$. Normalization is the largest contribution to the total systematics and it is expected to be fully correlated bin to bin.

- Feed-down induced by weak decays of strange particles is especially important for pions and protons (1-2.5\%) as well as neutrons (3\%). This is the second largest contribution to the systematics. For kaons, it can be neglected. It is expected to be uncorrelated bin to bin.

- Binning is the correction for finite bin width determining the deviation of the real cross-section at the center of the bin from the measured one. It is approximately $0.5 \%$. 


\begin{tabular}{|l|c|c|}
\hline particle produced & total systematic (quadratic sum) & total systematic (upper limit) \\
\hline$\pi^{ \pm}$ & $3.8 \%$ & $7.5 \%$ \\
$K^{ \pm}$ & $4.2 \%$ & $8.7 \%$ \\
backward protons & $4.7 \%$ & $10.0 \%$ \\
forward protons & $3.7 \%$ & $7.0 \%$ \\
neutrons & $10 \%$ & $28.0 \%$ \\
\hline
\end{tabular}

TABLE 4.2: NA49 systematic uncertainties.

- Trigger bias due to fact that secondary produced particles can hit the trigger and cause the event to be vetoed. This is approximately $0.5-1 \%$.

- Tracking efficiency: $0.5 \%$.

- Other uncertainties like detector absorptions, pion decays and reinteractions in the target are in total about $0.5 \%$.

- Other neutron uncertainties related to the reconstruction.

A summary of the NA49 systematics is shown in the Table 4.2.

\subsubsection{Barton et al.}

The result reported by Barton et al. [56] is a comprehensive cross-section measurements of fast secondaries (momentum of the particles produced in 30-88 $\mathrm{GeV} / \mathrm{c}$ ) with different incident beams (pions, kaons and protons) at $100 \mathrm{GeV}$ using the Fermilab Single Arm Spectrometer facility in the M6E beamline (see page 741775 in Ref. [72] for a description). The use of the same detector to study the A dependence of the invariant differential cross-sections cancels some of the detector performance systematic uncertainties.

The relevant Barton data for NuMI is the pion production from proton - carbon interactions at $100 \mathrm{GeV}$. However, there is a disagreement of the pion and proton 
production between Barton and NA49 in the data points where they overlap. We are going to explain later in this section the assumptions we made to use Barton data.

\subsubsection{MIPP thin target}

Ratios of high energy $K / \pi$ have been studied in [69] from proton - carbon interactions. As will be explained later, combining NA49 and MIPP thin target data allows us to extend the data to higher energy kaon production.

\subsubsection{Thin target pion production}

NA49 data is used to correct the pion invariant differential cross-sections in the $-0.1<x_{F}<0.5$ and $0<p_{T}<2 \mathrm{GeV} / \mathrm{c}$ range. Figure 4.25 shows some of the 270 bins per particle of NA49 data taken from [66]. The right plot corresponds to $\pi^{+}$ and the left one to $\pi^{-}$. Many data points populate the $x_{F}$ region below 0.15 and are not shown in the plots. The lines come from NA49 interpolation. We implement our own interpolation as described later.

Barton et al. ([56]) reported their pion invariant differential cross-section data for $x_{F}$ in $0.3-0.88$ and $p_{T}=0.3,0.5 \mathrm{GeV} / \mathrm{c}$. As was pointed out, Barton and NA49 are in disagreement in points where they overlap. Figure 4.26 (upper plot) shows both datasets for $0.3<x_{F}<0.5$ (MC values in the plot are just for reference). Barton cross-sections for $\pi^{+}$are approximately 25\% less than NA49 [66]. Similar differences are seen for $\pi^{-}$. However, the ratios $\pi^{+} / \pi^{-}$agree between both datasets. We use this fact to extend the NA49 data for $x_{F}>0.5$ by imposing a $25 \%$ reduction to Barton data and adding a 25\% uncertainty due to this assumption. Figure 4.26 (lower plot) also show $\pi^{+}$Barton data for $x_{F}>0.5$. 

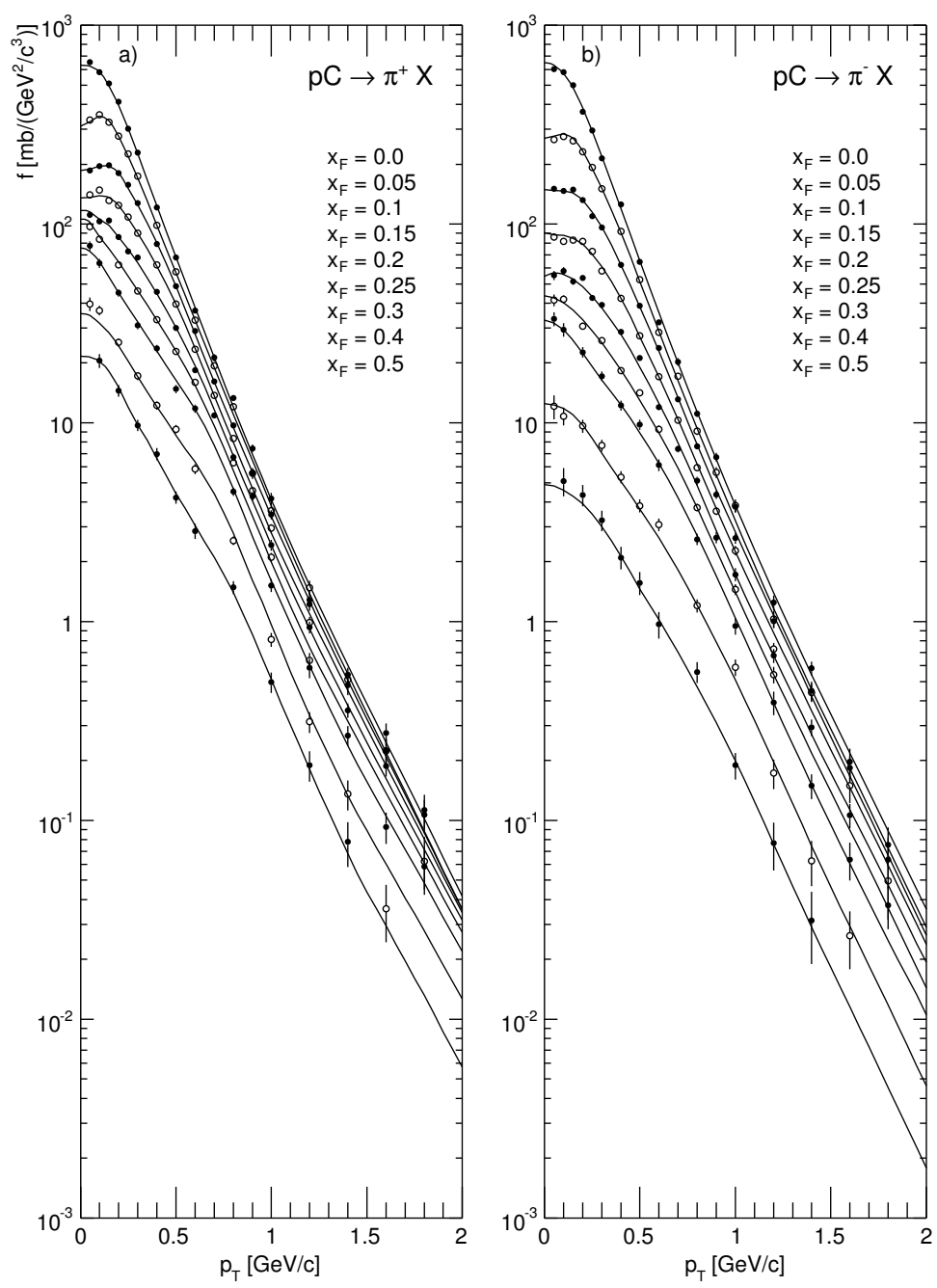

FIG. 4.25: NA49 charged pion production in proton carbon interactions. 


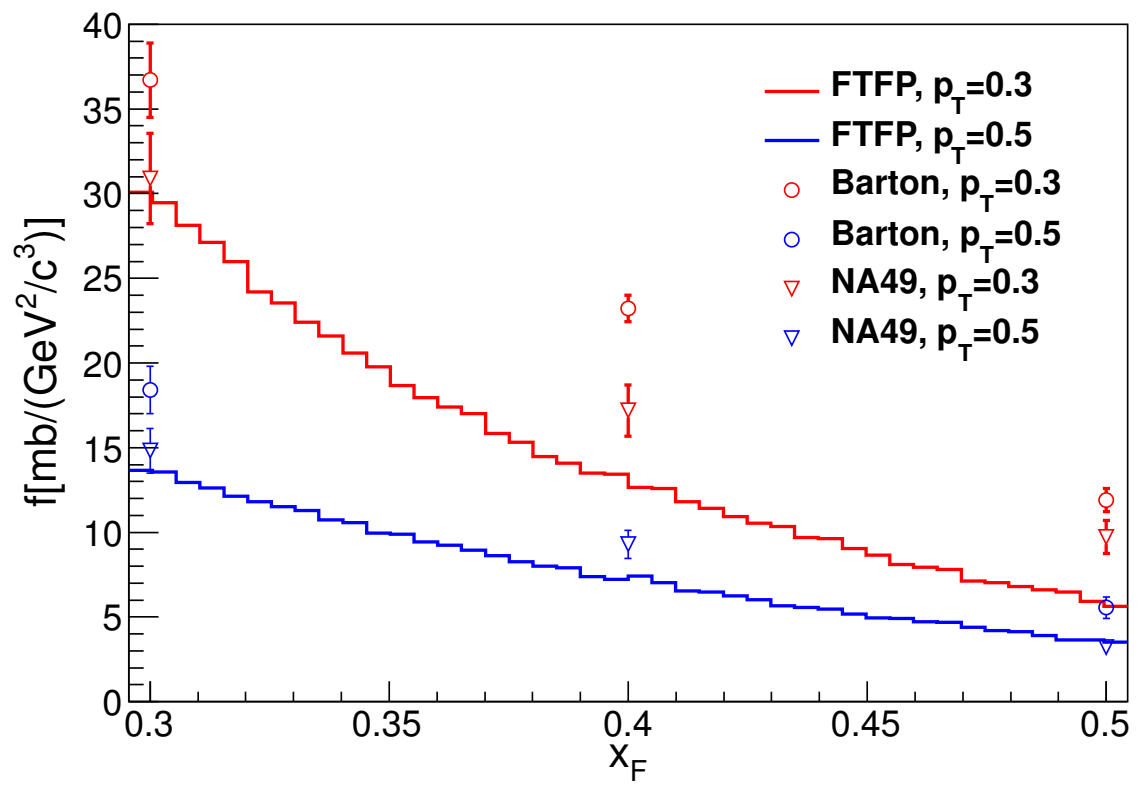

(a) Barton - NA49 comparison for $\pi^{+}$in $0.3<x_{F}<0.5$.

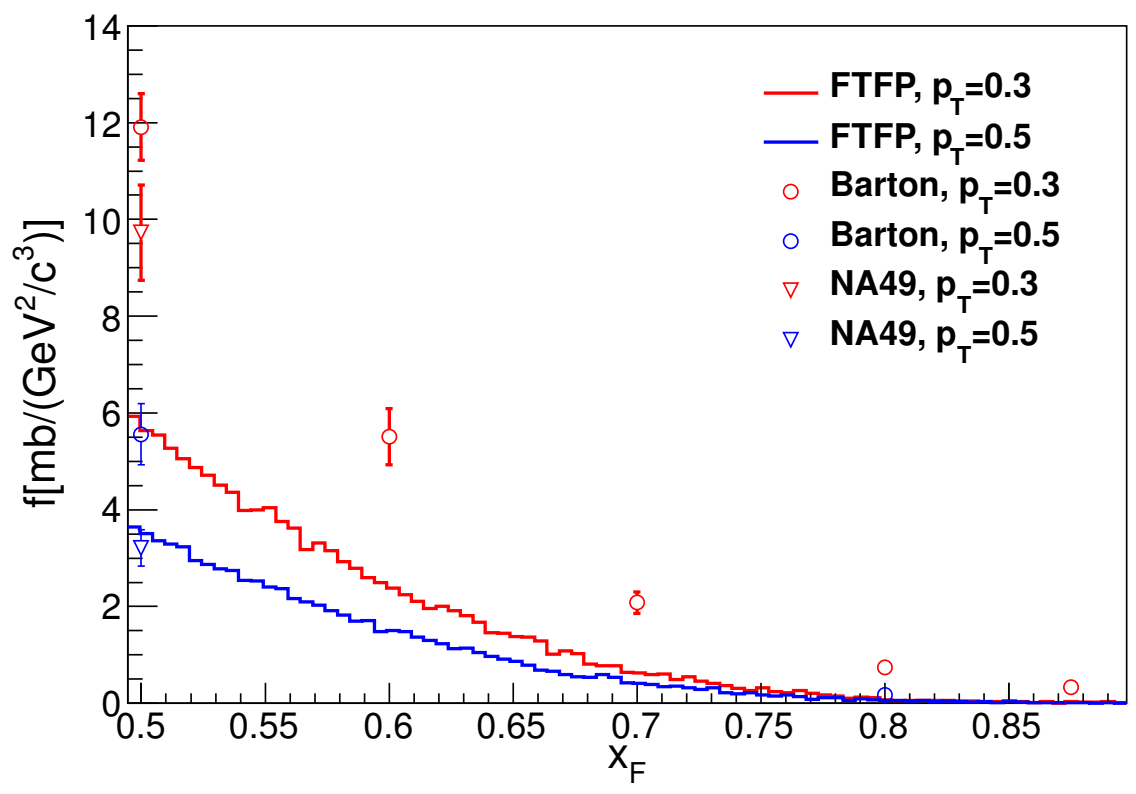

(b) $\pi^{+}$Barton data for $x_{F}>0.5$.

FIG. 4.26: Barton et al and NA49 $\pi^{+}$data in proton - carbon interactions. 


\section{Technical details of the implementation}

The implementation of the pion production correction is as follows:

1. The NA49 and Barton data are interpolated to smooth transitions between bins. The granularity is $\Delta x_{F}=0.005$ and $\Delta p_{T}=0.025 \mathrm{GeV} / \mathrm{c}$. This is finer than the data binning. MC values have the same granularity.

2. The statistical and systematic uncertainties assigned to each bin is the closest fractional error value of NA49 or Barton datasets.

3. The statistical uncertainties are assumed to be uncorrelated. Figures in 4.27 show these uncertainties for the interpolated NA49 data. The region for low $x_{F}$ (0-0.25) and $p_{T}<0.8 \mathrm{GeV} / \mathrm{c}$ has small errors (less than 5\%) for both $\pi^{+}$and $\pi^{-}$. This is important to determine the flux focusing peak of MINERvA and NOvA as can be seen in Chapters 5-7.

4. The systematic uncertainties are assumed to be $100 \%$ correlated between bins. It is clearly an overestimated value since only the normalization uncertainty is known to be fully correlated. A computational complication leads to this decision: the calculation of the Cholesky decomposition of a big matrix (for instance, in the case of NA49: $121 x_{F}$ bins $\times 81 p_{T}$ bins makes a 9801-dimension matrix to be handled ${ }^{7}$. Equation 4.23 demands that we compute the Cholesky decomposition of the covariance matrix. The total systematic uncertainties (see Table 4.2) added in quadrature is used.

5. An energy scaling correction is applied to use these datasets for any incident proton on carbon in $12-120 \mathrm{GeV} / \mathrm{c}$.

\footnotetext{
${ }^{7}$ Some techniques can be used to deal with this complication but a simple assumption had to be made due to the timescale to present the results of this analysis.
} 


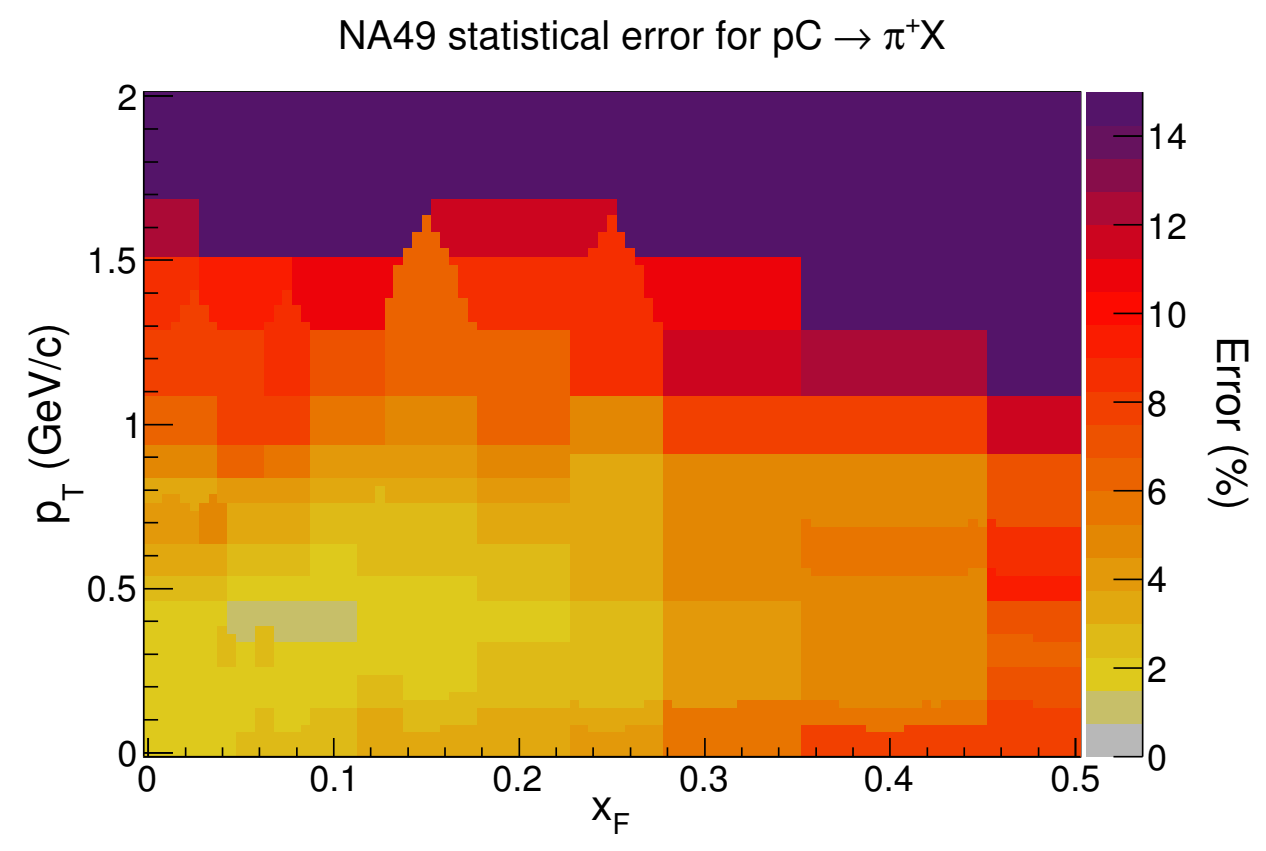

(a) $\pi^{+}$.

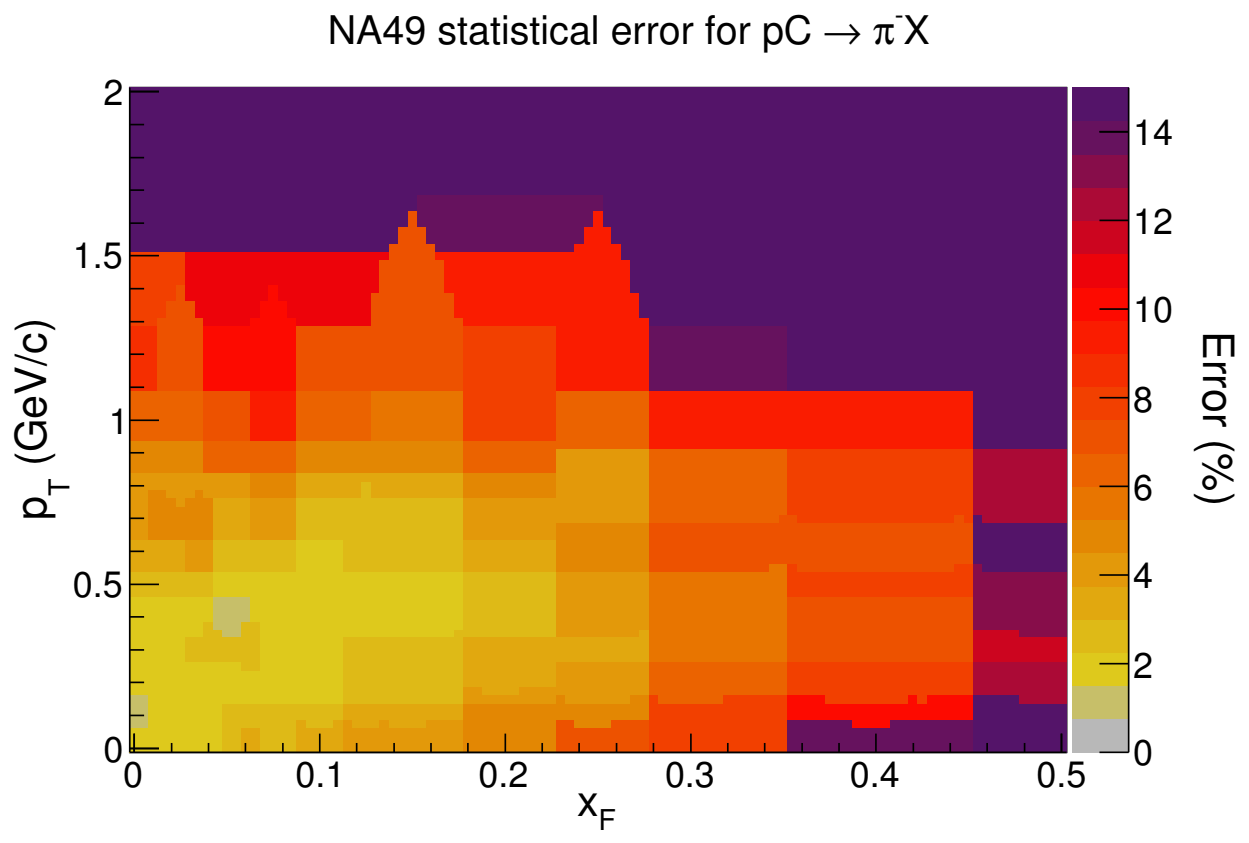

(b) $\pi^{-}$.

FIG. 4.27: NA49 statistical error for pion production. 
Figures in 4.28 show the weights for $\pi^{+}$and $\pi^{-}$applying Equation 4.8. For $x_{F}<$ $0.3, \pi^{+}$below $p_{T}=0.4 \mathrm{GeV} / \mathrm{c}$ are underestimated by the $\mathrm{MC}$ and overestimated otherwise. For $\pi^{-}$, almost the entire region for $p_{T}<1 \mathrm{GeV} / \mathrm{c}$ has to correct the MC up.

Pion production from neutron - carbon interactions was also corrected with the NA49 and Barton data using Equation 4.20.

\subsubsection{Thin target kaon production}

NA49 data is used to correct the invariant differential cross-sections for $x_{F}$ in [0,0.2] as reported by [68] (figures $4.29 \mathrm{a}$ and $4.29 \mathrm{~b}$ for $K^{+}$and $K^{-}$, respectively). The lines are interpolated values in Ref. [68]. We make our own interpolation. Except for low $p_{T}$, the coverage is good for low $x_{F}$ kaons.

A combination of NA49 pion [66] production with MIPP $K / \pi([69])$ is used to extend the kaon data beyond $x_{F}=0.2$. Figure 4.30 shows the MIPP kaon ratios. We are interested in the right hand side plots. The binning convention is described in the figure caption.

\section{Technical details of the implementation}

The charged kaon production correction uses Equation 4.11 as in the charged pions case and its implementation is as follows:

1. As for pion production, NA49 kaon production data is interpolated to smooth transitions between bins. The granularity is finer than the data binning: $\Delta x_{F}=$ 0.005 and $\Delta p_{T}=0.025 \mathrm{GeV} / \mathrm{c}$. However, no interpolation is made for MIPP pC ratios. MC values have the same granularity as their corresponding data after interpolation. 


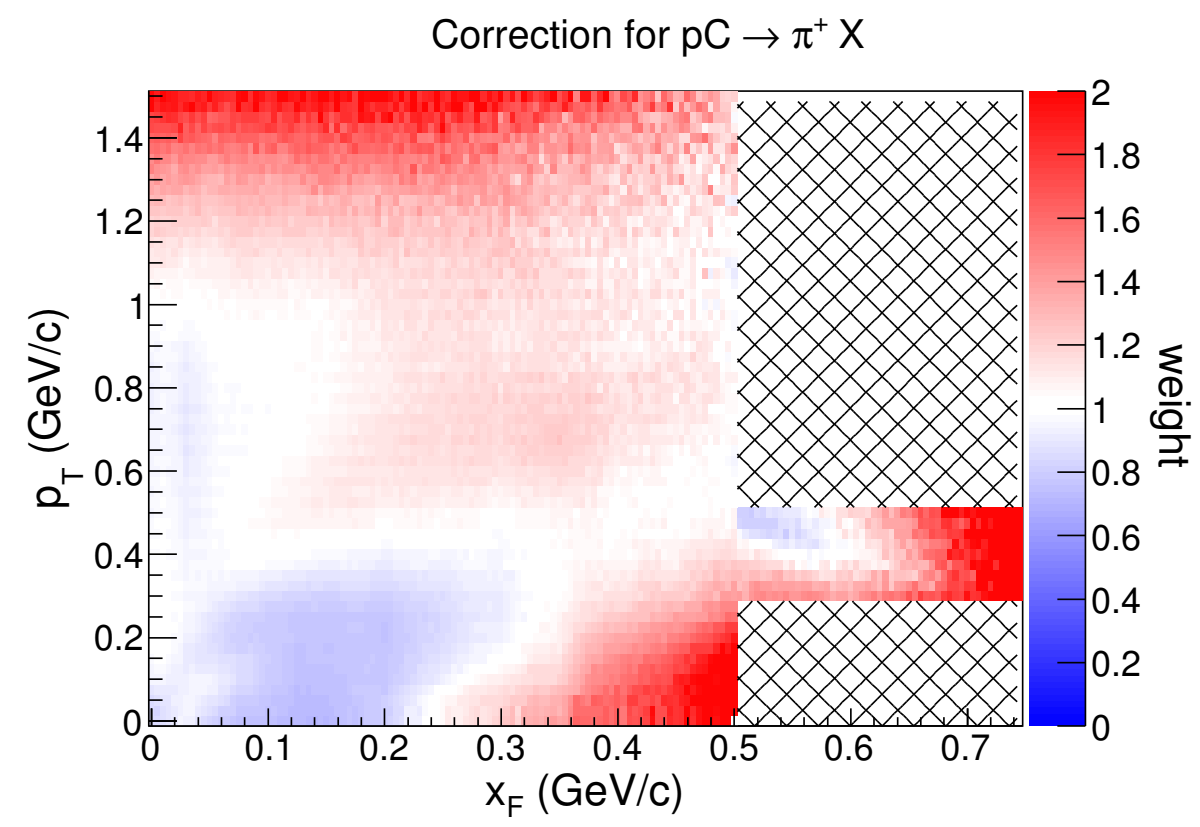

(a) $\pi^{+}$.

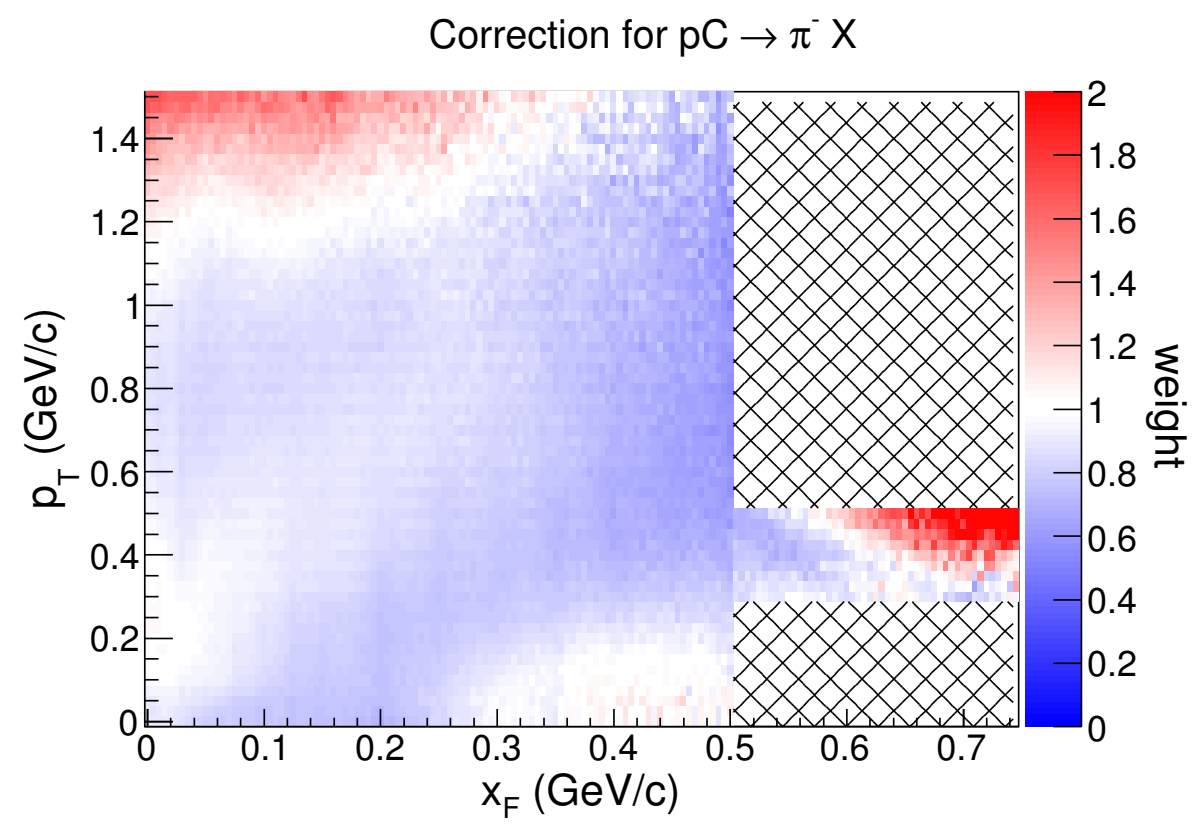

(b) $\pi^{-}$.

FIG. 4.28: Thin target weights for charged pions. 


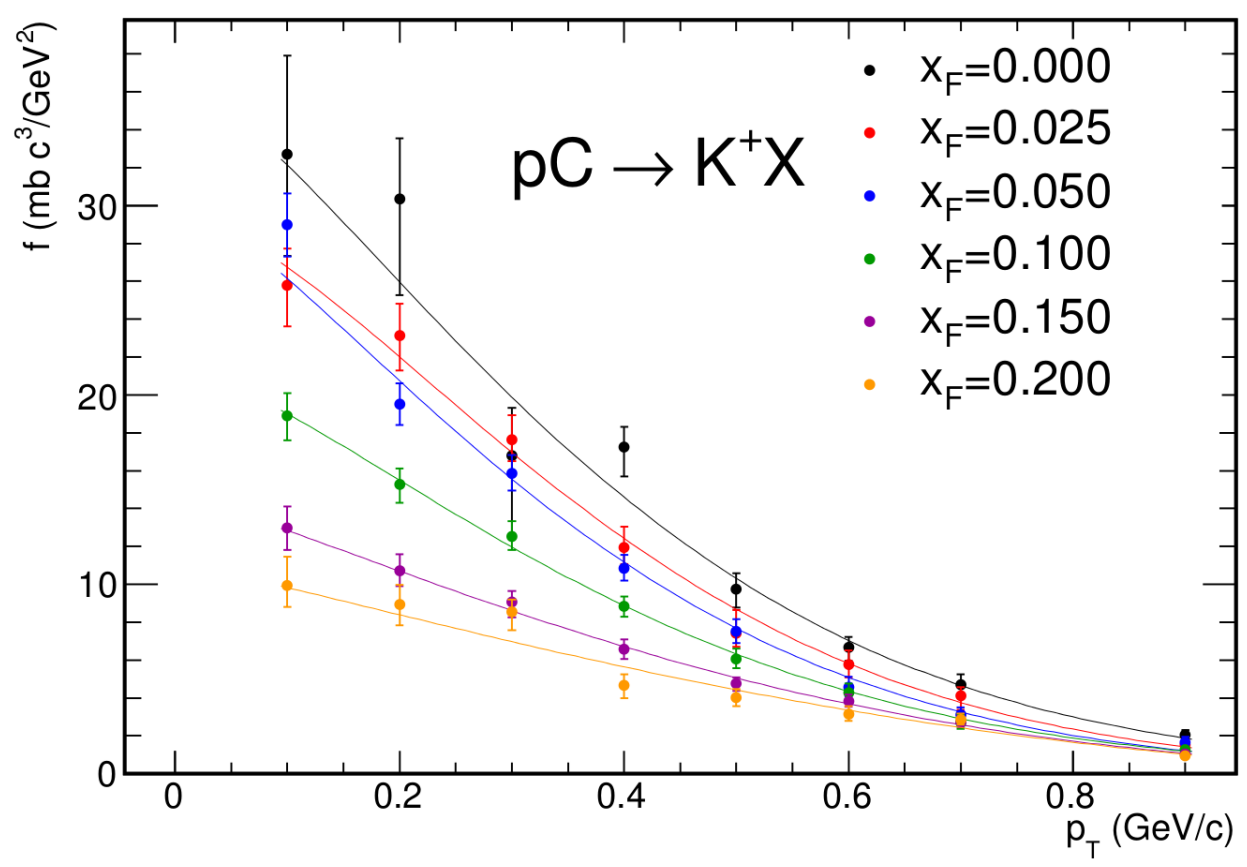

(a) $K^{+}$.

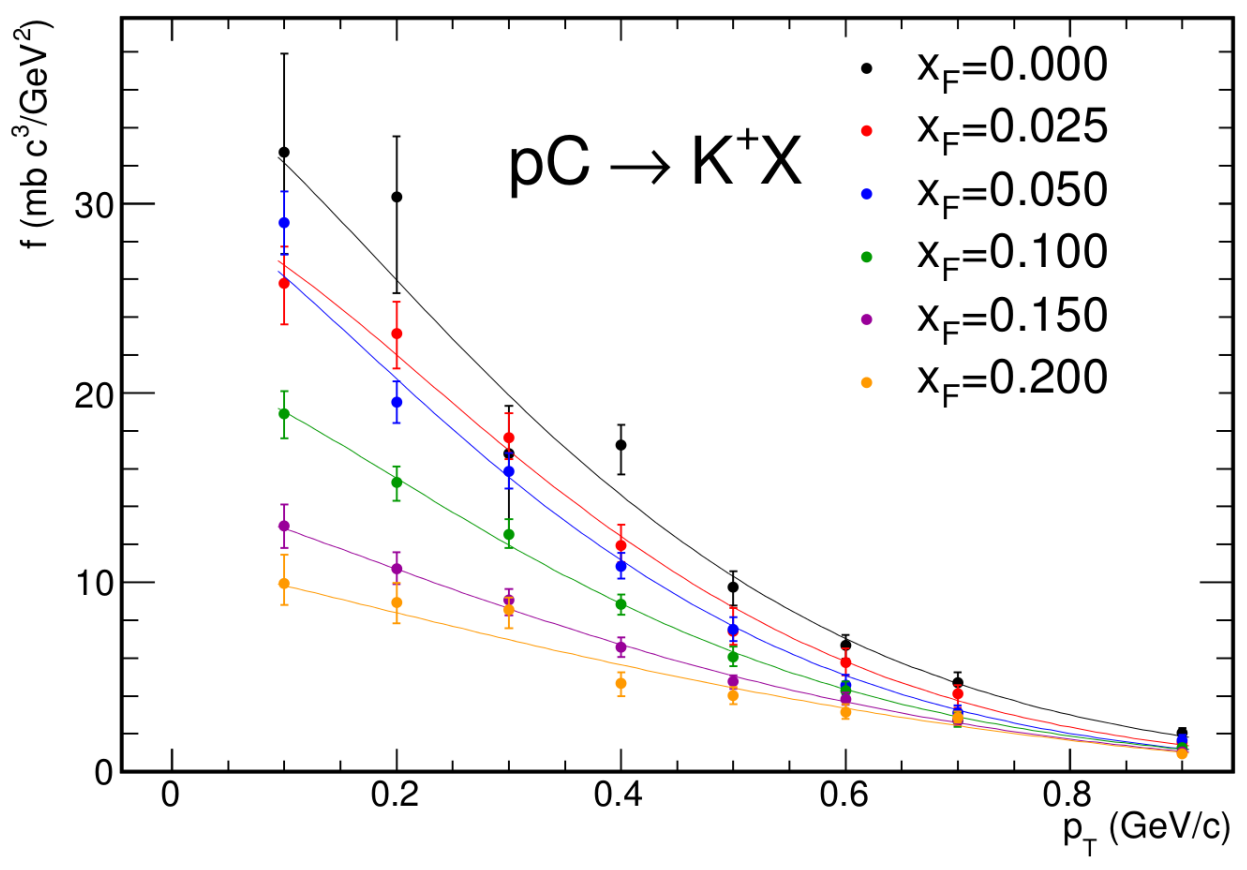

(b) $K^{-}$.

FIG. 4.29: NA49 invariant differential cross-section of charged kaon production in proton carbon interactions [68]. 

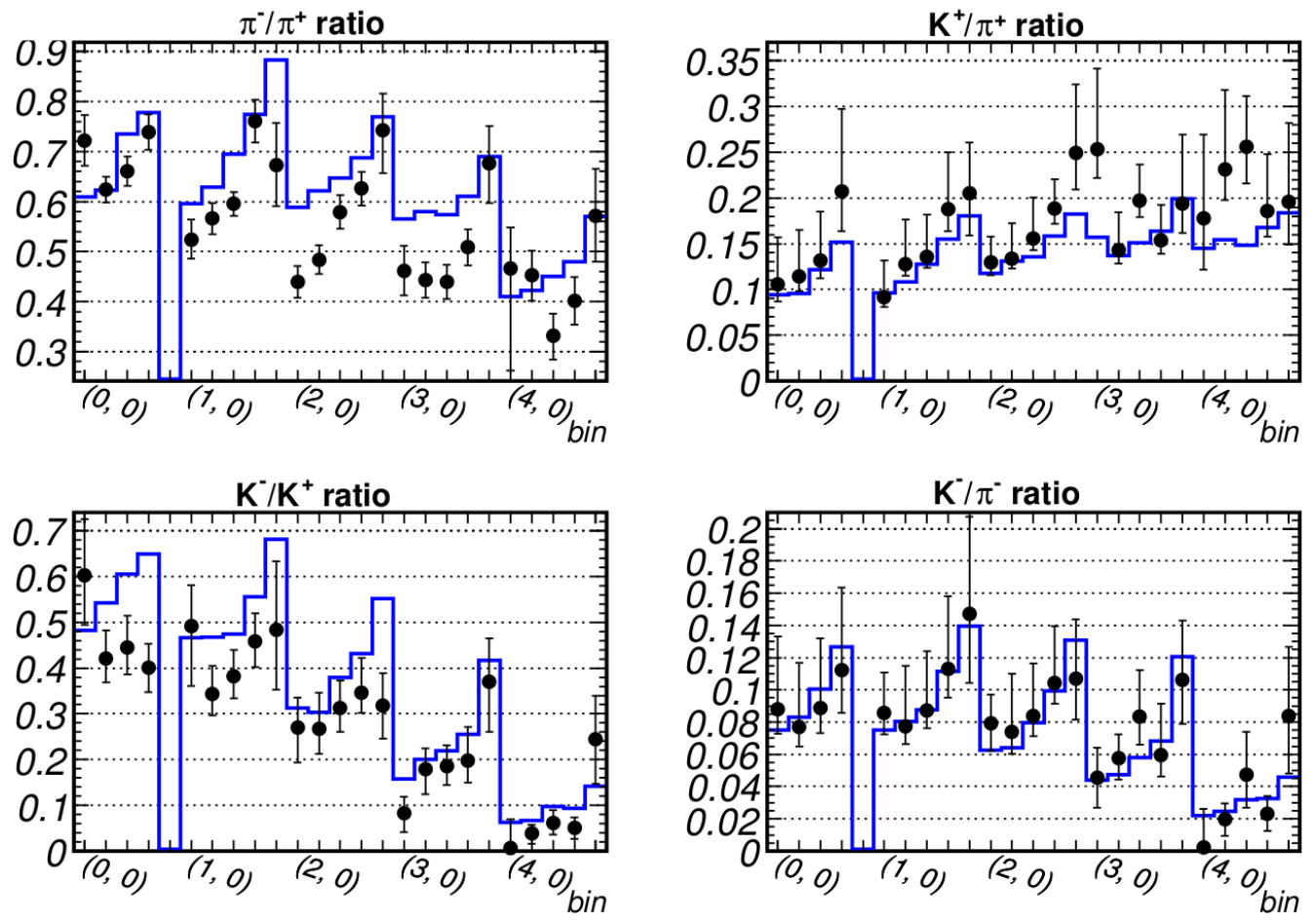

FIG. 4.30: MIPP $\pi^{-} / \pi^{+}, K^{-} / K^{+}, K^{+} / \pi^{+}$and $K^{-} / \pi^{-}$ratios. The momentum bin convention $(\mathrm{X}, \mathrm{Y})$ is such that $\mathrm{X}$ represent the longitudinal momentum and $\mathrm{Y}$ the transverse momentum. X $(\mathrm{GeV} / \mathrm{c})=0$ (20-24), 1 (24-31), 2 (31-42), 3 (42-60), 4 (60-90). Y(GeV/c) $=0(<0.2), 1(0.2-0.4), 2(0.4-0.6), 3(0.6-1.0), 4(>1.0)$. Plots taken from [69]. 
2. The statistical and systematic uncertainties assigned to each NA49 bin is the closest fractional data error. For MIPP, the data uncertainty is used directly.

3. The statistical uncertainties are assumed to be uncorrelated. The NA49 statistical uncertainties for $K^{+}$and $K^{-}$production are shown in figures in 4.31. The uncertainties are typically higher than pions. The statistical uncertainties for MIPP $K / \pi$ ratios are in figures at 4.32 with the exact value written in each bin.

4. Consistent with the pion correction, the NA49 systematic uncertainties are assumed to be $100 \%$ correlated between bins. The total of the systematic uncertainties (see Table 4.2) added in quadrature is used for NA49. Figure 4.33 shows the systematic uncertainties used in MIPP thin target $K / \pi$ ratios. We do not assume any bin to bin correlation for MIPP data ratios.

5. The energy scaling correction is applied for any incident proton on carbon with energies of $12-120 \mathrm{GeV} / \mathrm{c}$.

6. Formula 4.21 is used to estimate the neutral kaon cross-section from charged kaon data. The MC $K_{L}^{0}$ and $K_{S}^{0}$ yields are calculated off-line.

7. The factors $y_{D a t a}$ and $y_{M C}$ are calculated as in the charged pion case.

Figures $4.34 \mathrm{a}$ and $4.34 \mathrm{~b}$ and shows that the weights for $K^{+}$and $K^{-}$, respectively. Neutral kaons weights are shown in figures at 4.35 .

\subsubsection{Thin target nucleon production}

Proton production in pC interactions has been measured in NA49 [67] as invariant double differential cross-sections for $-0.8<x_{F}<0.95$ and $p_{T}<2 \mathrm{GeV} / \mathrm{c}$. Protons produced quasi-elastically have Feynman-x greater than 0.95 and they are not part of this data since those events are always vetoed by the NA49 trigger 


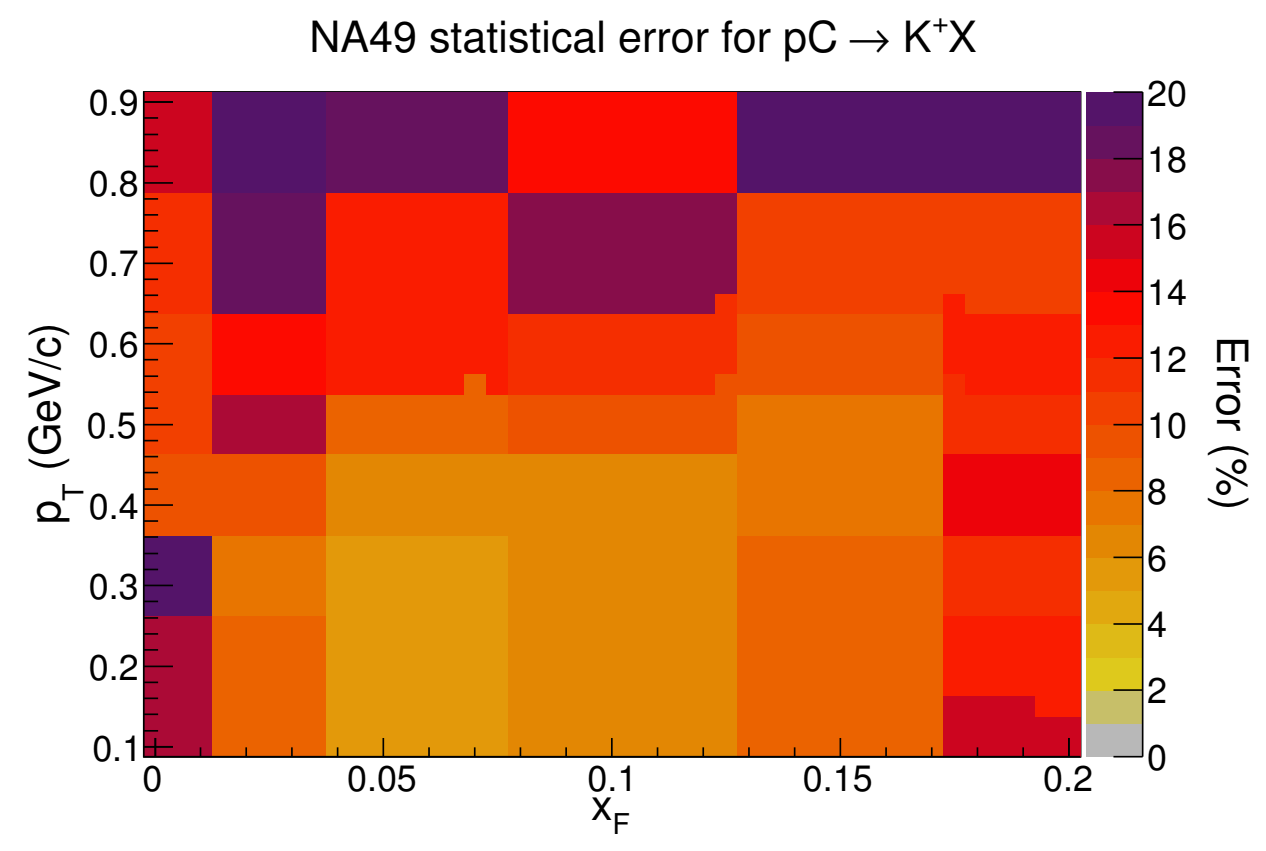

(a) $K^{+}$.

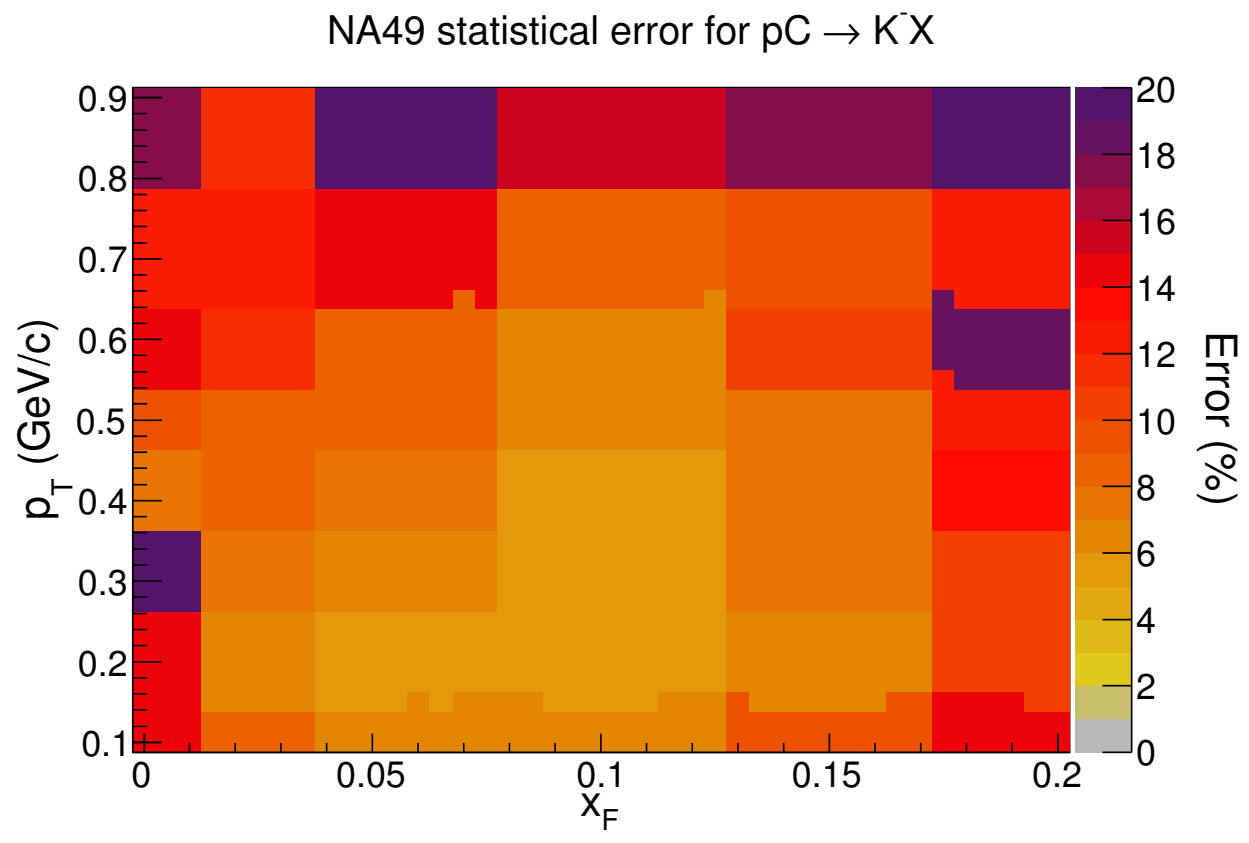

(b) $K^{-}$.

FIG. 4.31: NA49 statistical uncertainties for charged kaon production. 


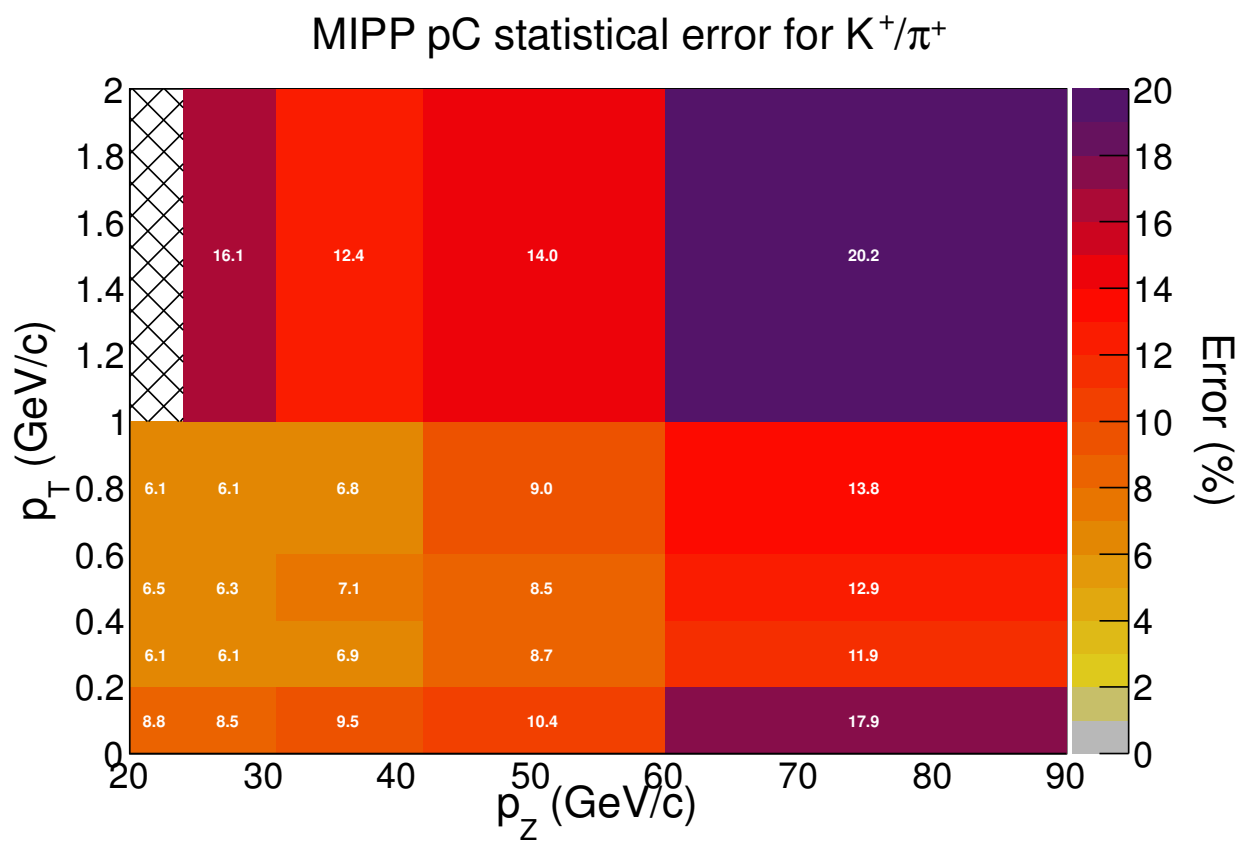

(a) $K^{+} / \pi^{+}$

MIPP pC statistical error for $\mathrm{K}^{-} / \pi^{-}$

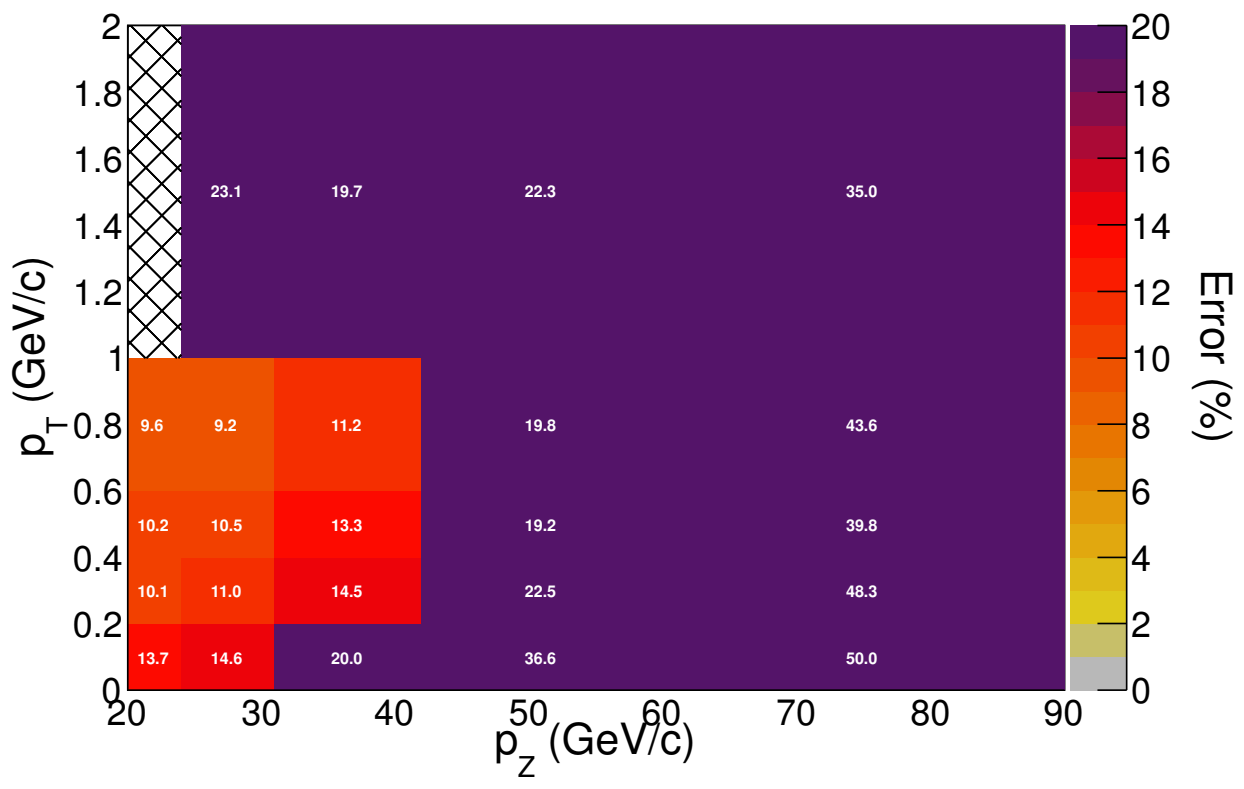

(b) $K^{-} / \pi^{-}$.

FIG. 4.32: MIPP pC statistical uncertainties for $K / \pi$. 


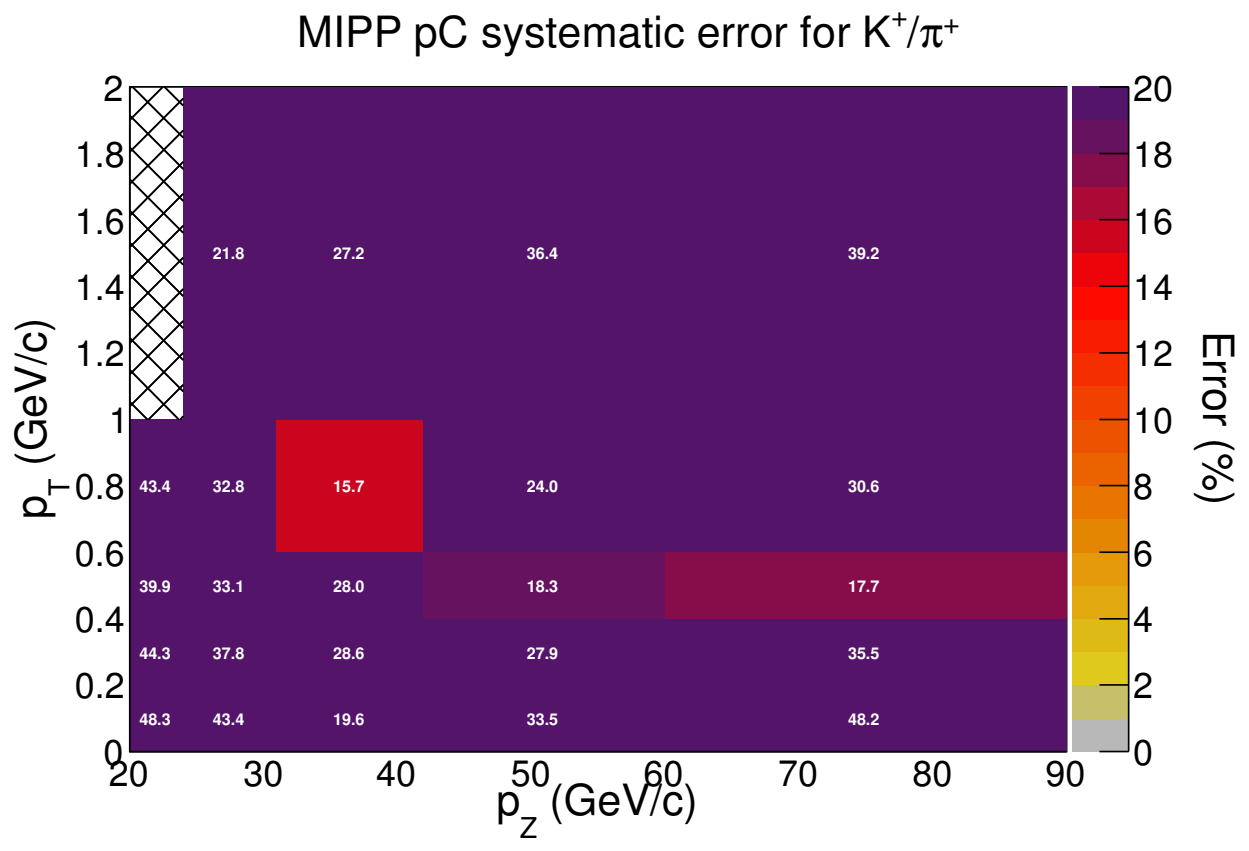

(a) $K^{+} / \pi^{+}$

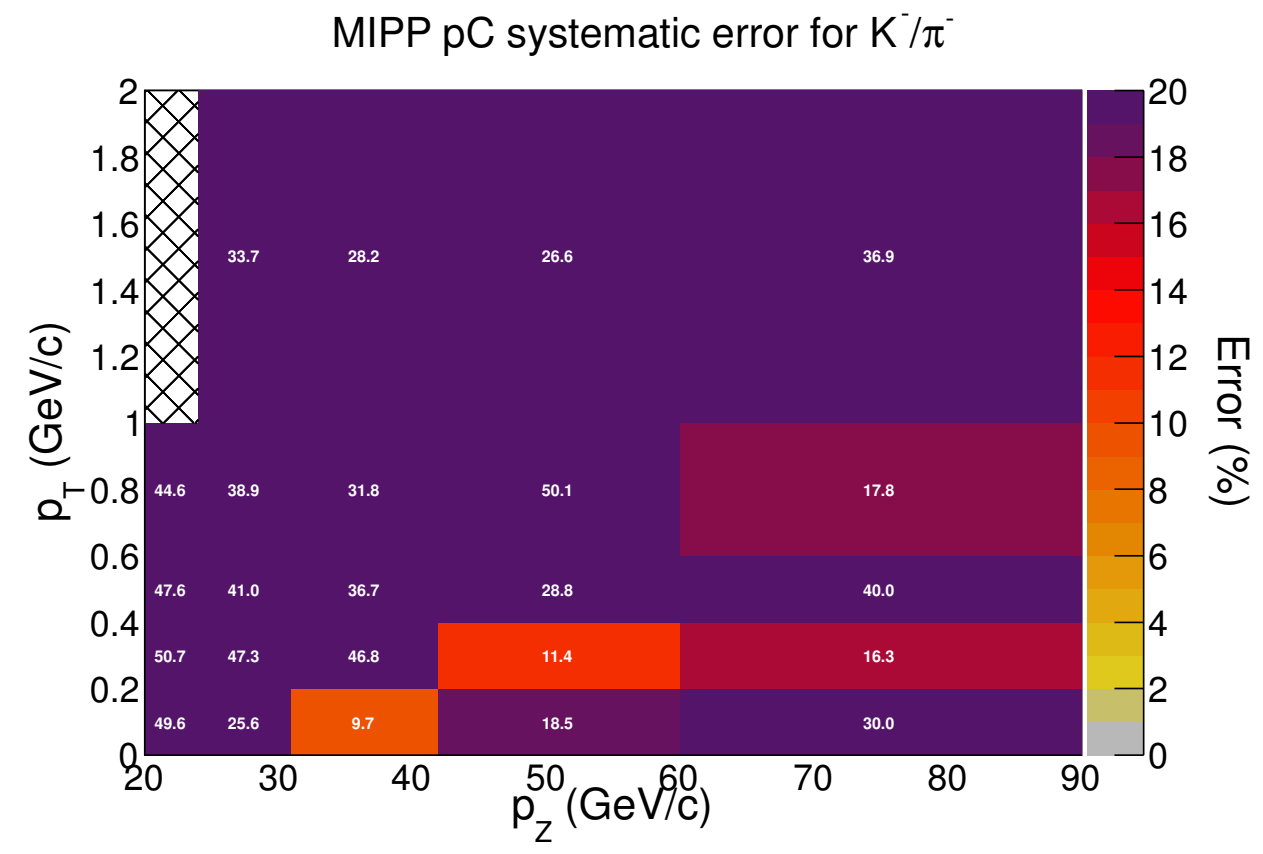

(b) $K^{-} / \pi^{-}$.

FIG. 4.33: MIPP pC systematic uncertainties for $K / \pi$. 


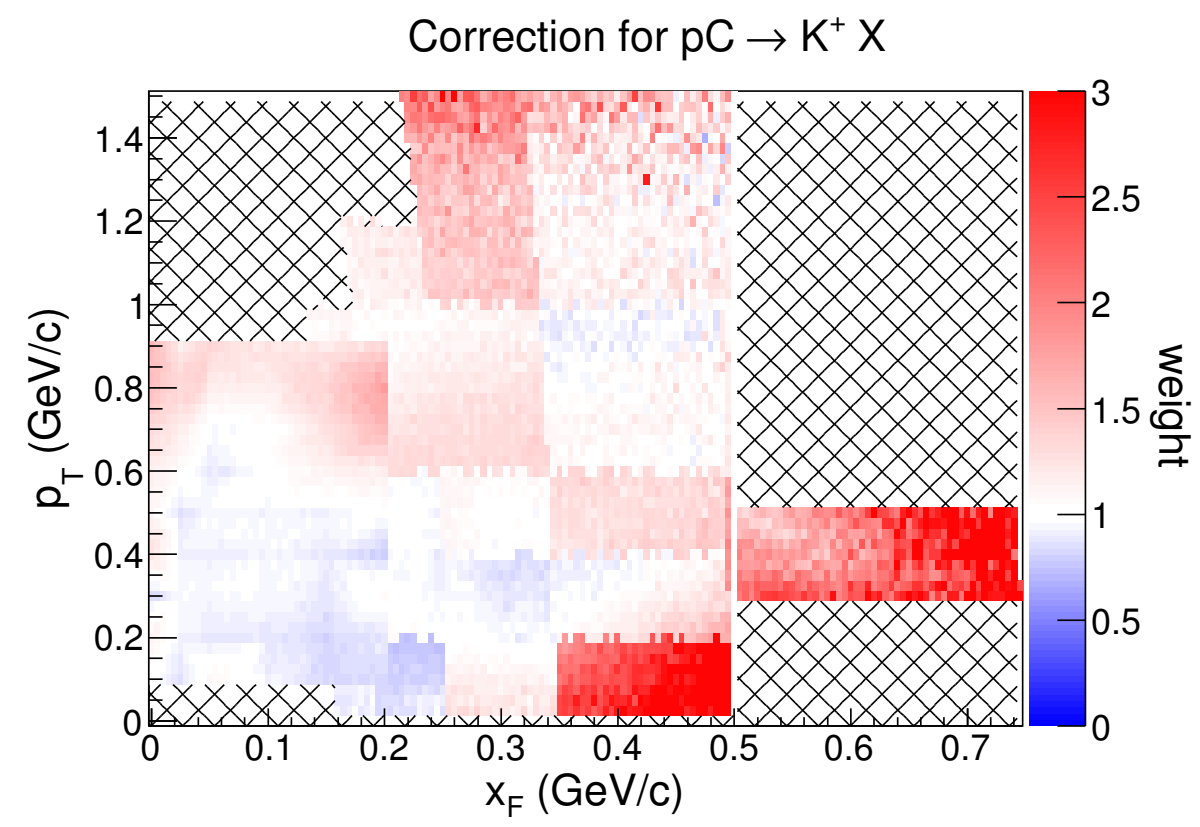

(a) $K^{+}$.

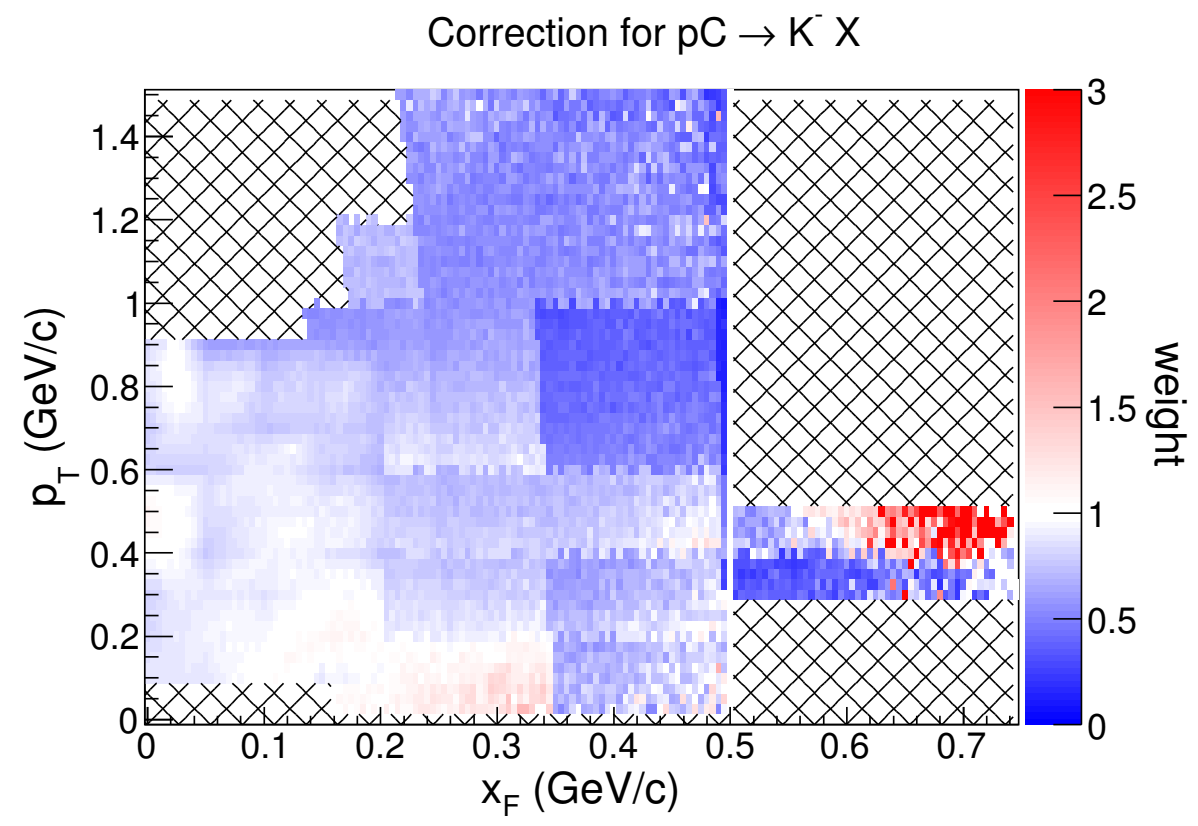

(b) $K^{-}$.

FIG. 4.34: Thin target weights for charged kaons. 


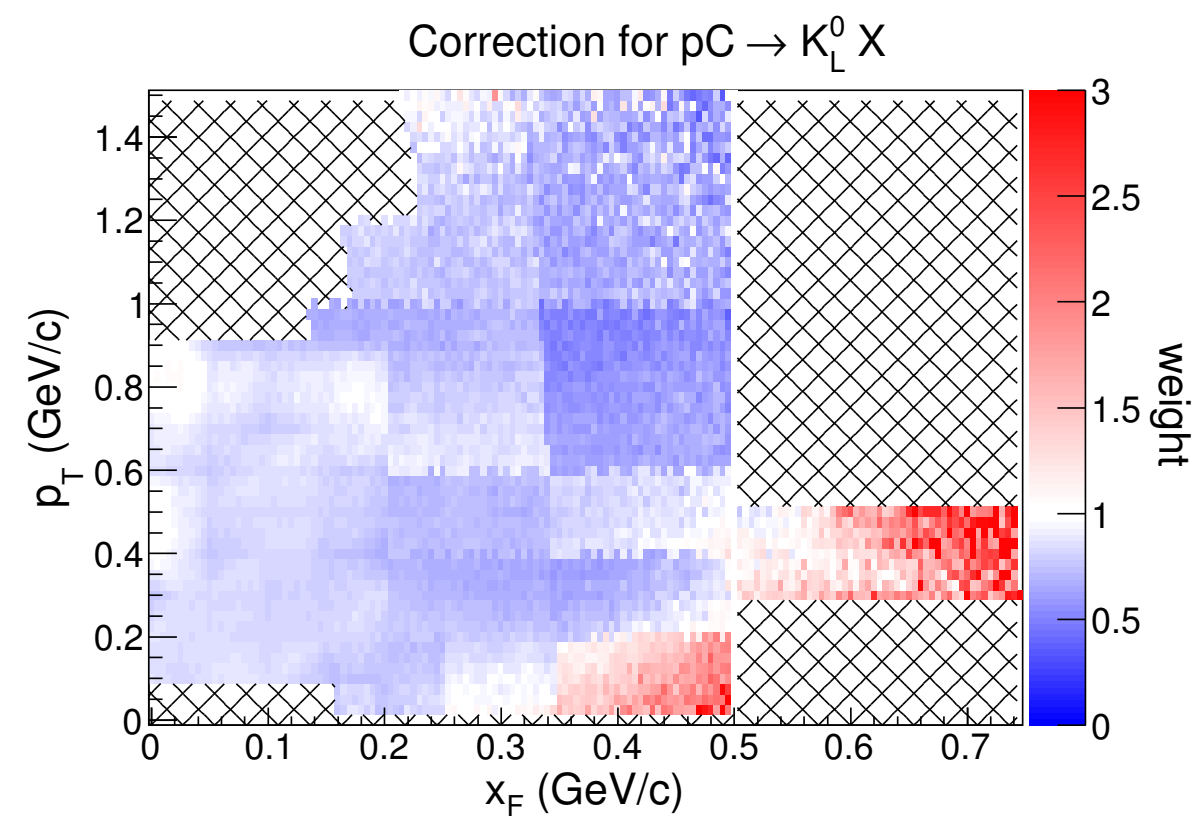

(a) $K_{L}^{0}$.

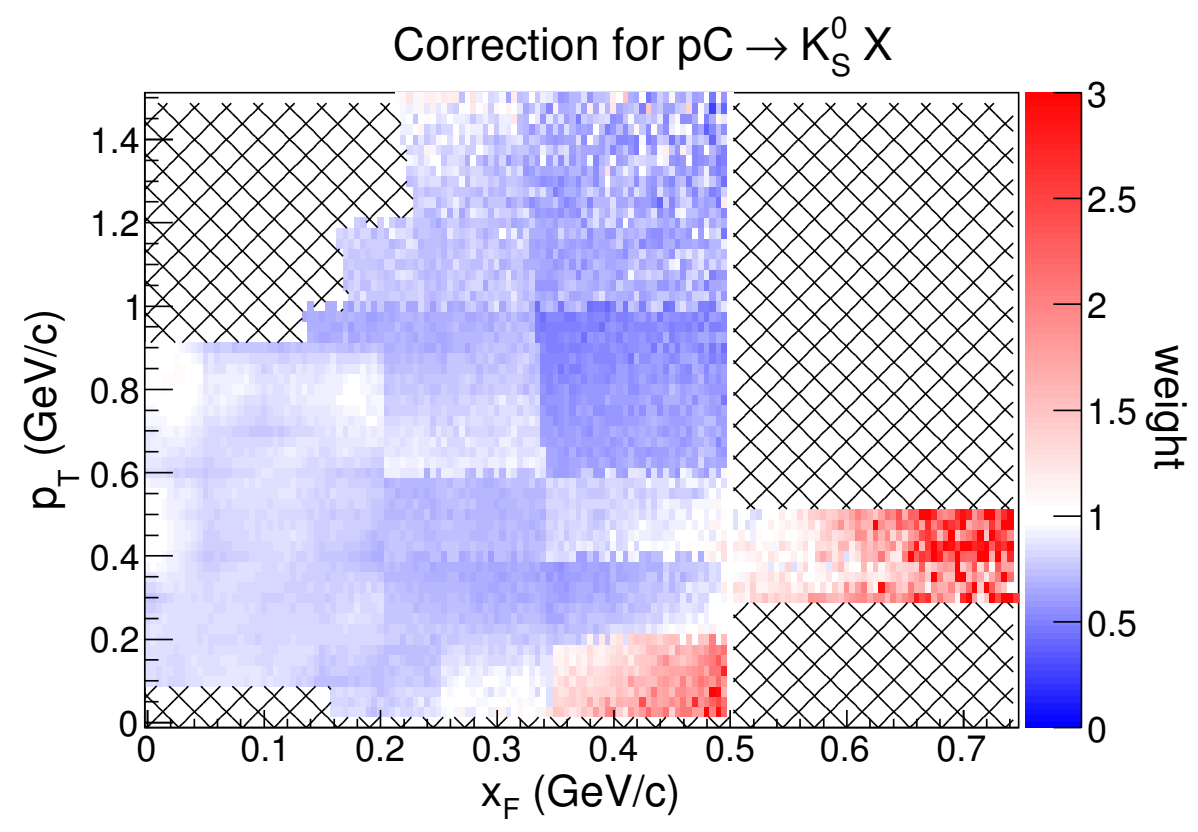

(b) $K_{S}^{0}$.

FIG. 4.35: Thin target weights for neutral kaons. 
counter. Figures in 4.36 show the data for proton (left side) and anti-proton production (right side) taken from Ref. [67]. Only the proton cross-section correction has been used since the anti-protons have very small effect. The values for negative $x_{F}$ are not shown in the figure, and the lines correspond to the NA49 interpolation reported in the paper. We have made our own interpolation.

NA49 also measured neutron production yields $\left(d n / d x_{F}\right)$ integrated over the $p_{T}$ acceptance for $0.1<x_{F}<0.9$. Figure 4.37 shows the 8 data points released by NA49 [67].

\section{Technical details of the implementation}

The implementation of the nucleon production correction is as follows:

- Proton production data has been interpolated with granularity of $\Delta x_{F}=0.005$ and $\Delta p_{T}=0.05 \mathrm{GeV} / \mathrm{c}$. The neutron yield was interpolated with a $4^{\text {th }}$ degree polynomial and sliced in bins with granularity of $\Delta x_{F}=0.005$.

- The statistical and systematic uncertainties assigned to each NA49 bin after interpolation are the closest fractional data uncertainty.

- Figure 4.38a shows the statistical uncertainties of proton production. The values are typically less than $5 \%$ for $p_{T}<1 \mathrm{GeV} /$ c. For neutron yield, the statistical error is around $10-15 \%$ (Figure $4.38 \mathrm{~b}$ ).

- The total systematic uncertainties added in quadrature (Table 4.2) and $100 \%$ correlation bin to bin are assumed. For protons, this is necessary because of the high number of bins that enter into the Cholesky decomposition. For neutrons this is not needed but it is made anyway for consistency with the other datasets used. Figures in $4.38 \mathrm{a}$ and $4.38 \mathrm{~b}$ show the fractional uncertainties for proton and neutron production, respectively. 

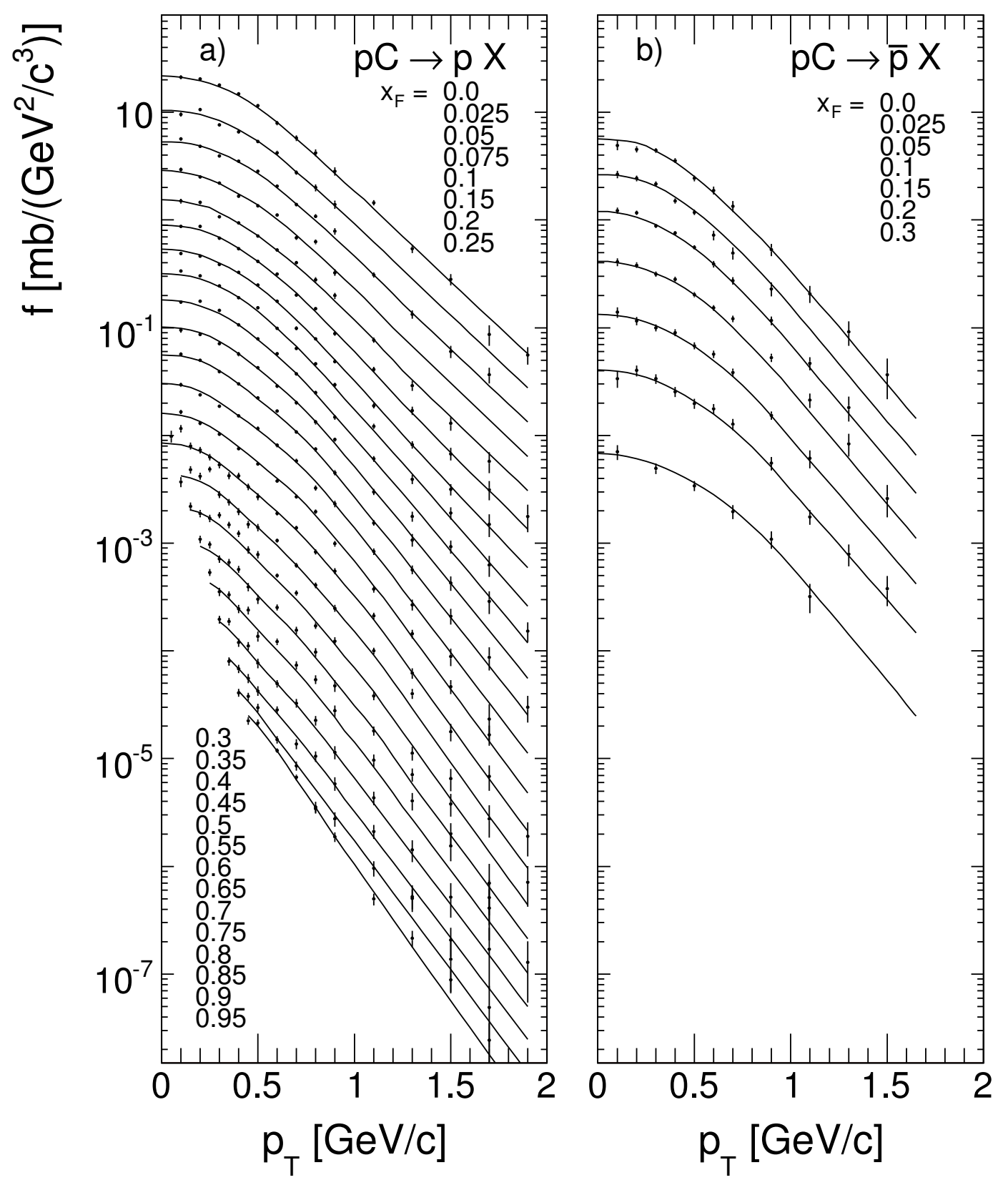

FIG. 4.36: NA49 invariant differential cross-section for proton production in proton carbon interactions. Plot taken from [67]. 


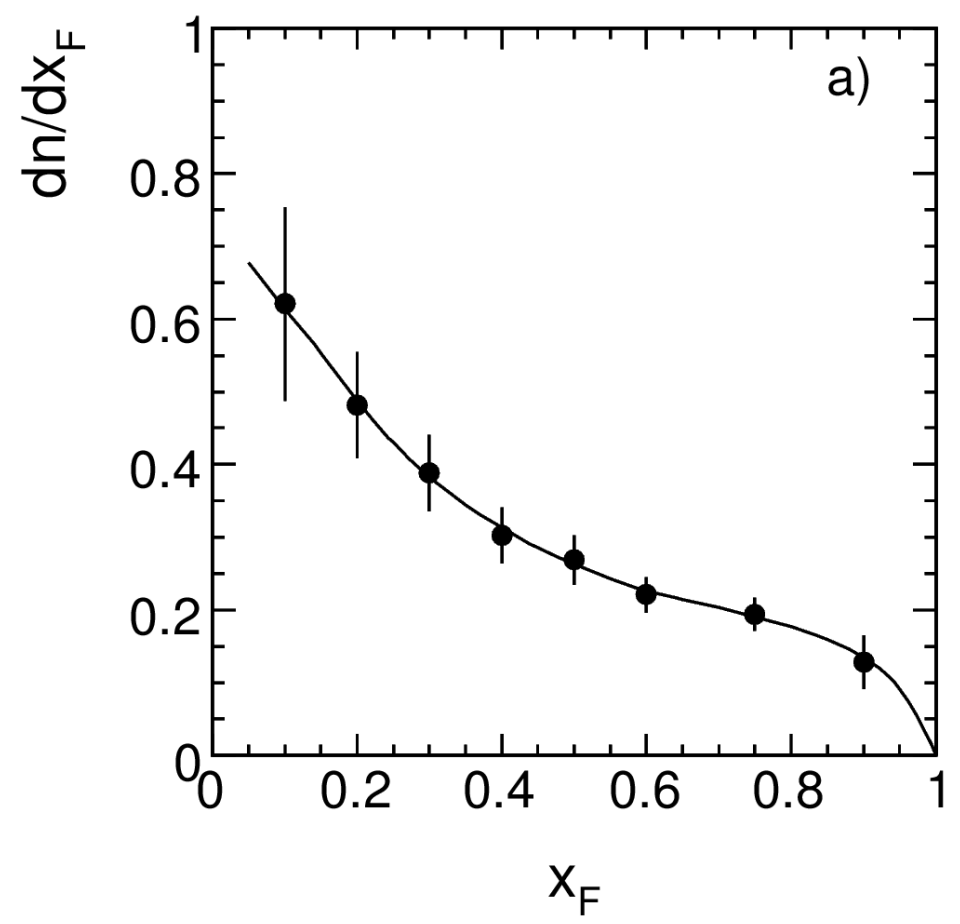

FIG. 4.37: NA49 neutron production yields in proton carbon interactions. Plot taken from $[67]$.

- As in pions and kaons, an energy scaling correction is used for nucleon production to go from $158 \mathrm{GeV}$ incident proton momentum to $12-120 \mathrm{GeV} / \mathrm{c}$.

The central part of the proton production cross-section correction is overestimated and has to be corrected down as can be seen in Figure 4.39a. The neutron yield needs to be corrected down for $0.15<x_{F}<0.65$ and up otherwise (Figure $4.39 b)$.

\subsection{Thin target data extension in Generation 2}

This section deals with the cases when we do not have data to apply directly in Generation 2. As was described in Section 4.2.3, the first approach can be to look if there are any theoretically guided data extensions and then use our best guess anchored in data. See Section 4.2.4. In any case, Generation 2 tries to avoid the 


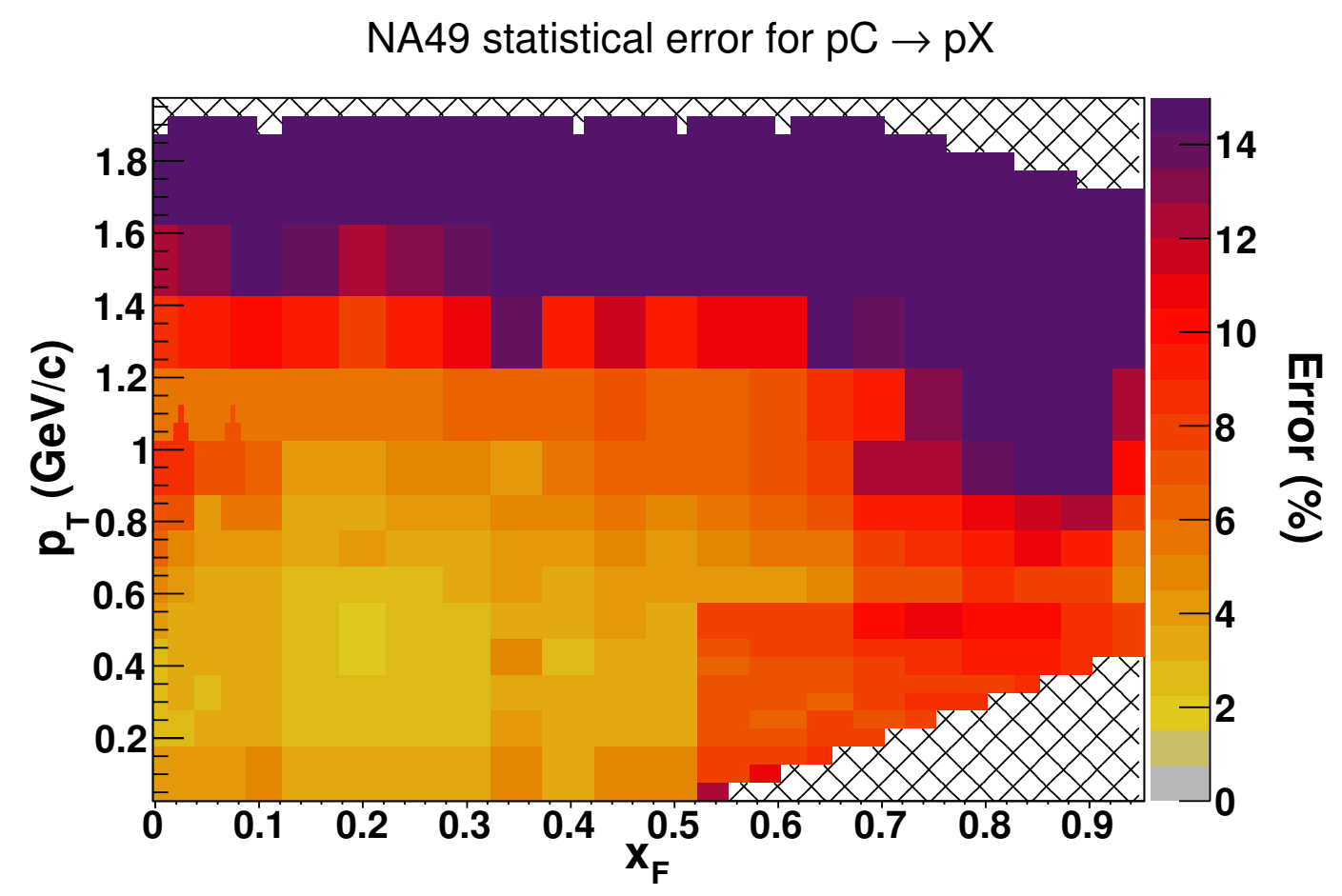

(a) proton

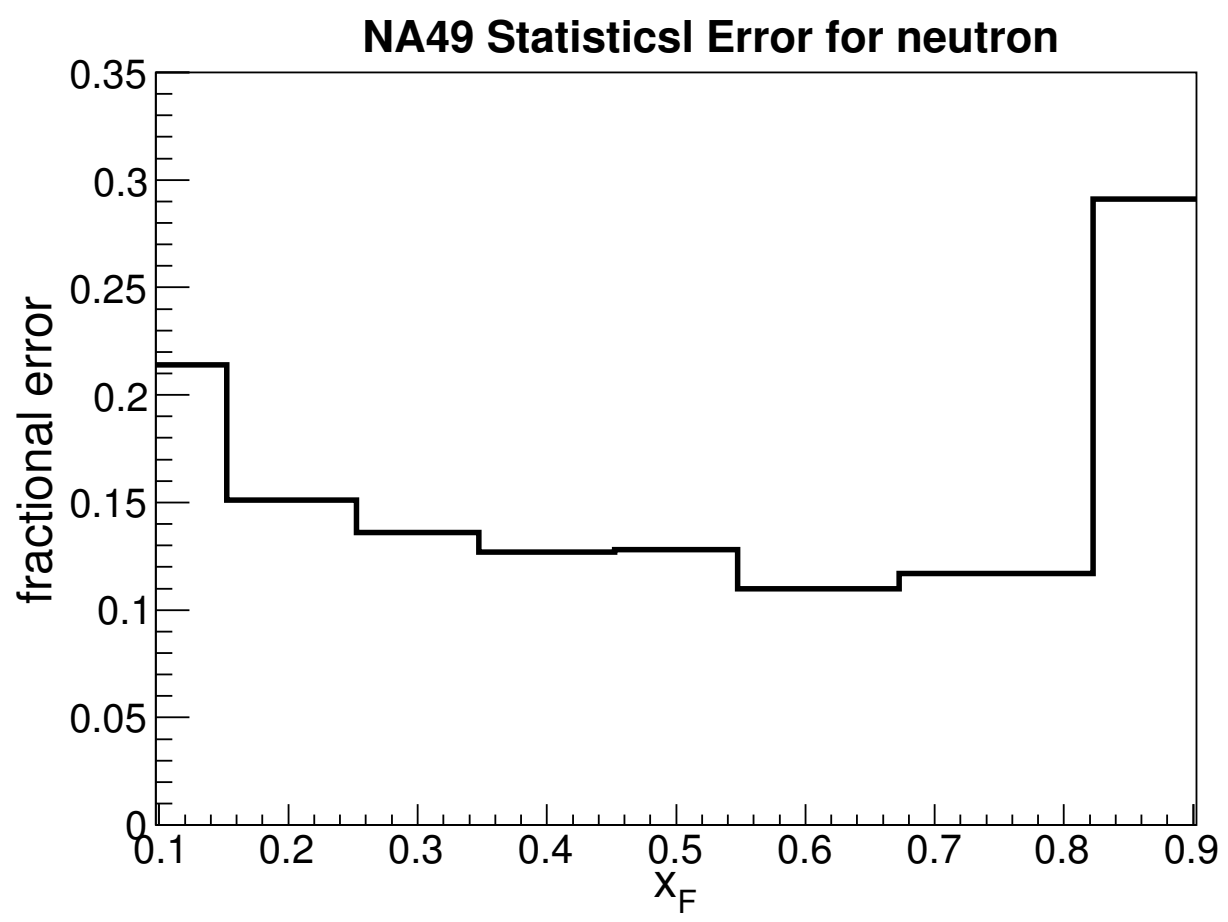

(b) neutron.

FIG. 4.38: NA49 nucleon statistical uncertainties. 


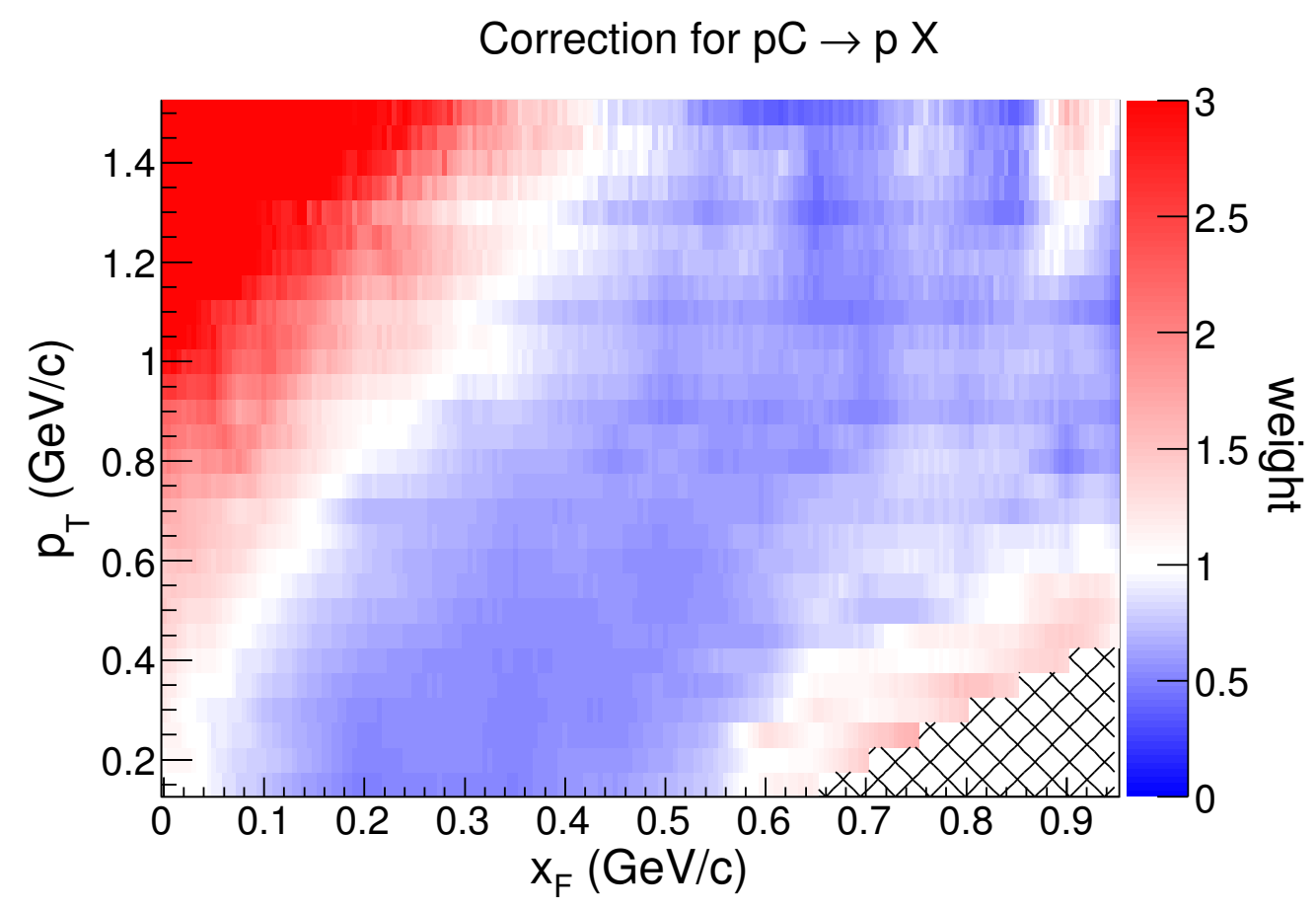

(a) protons.

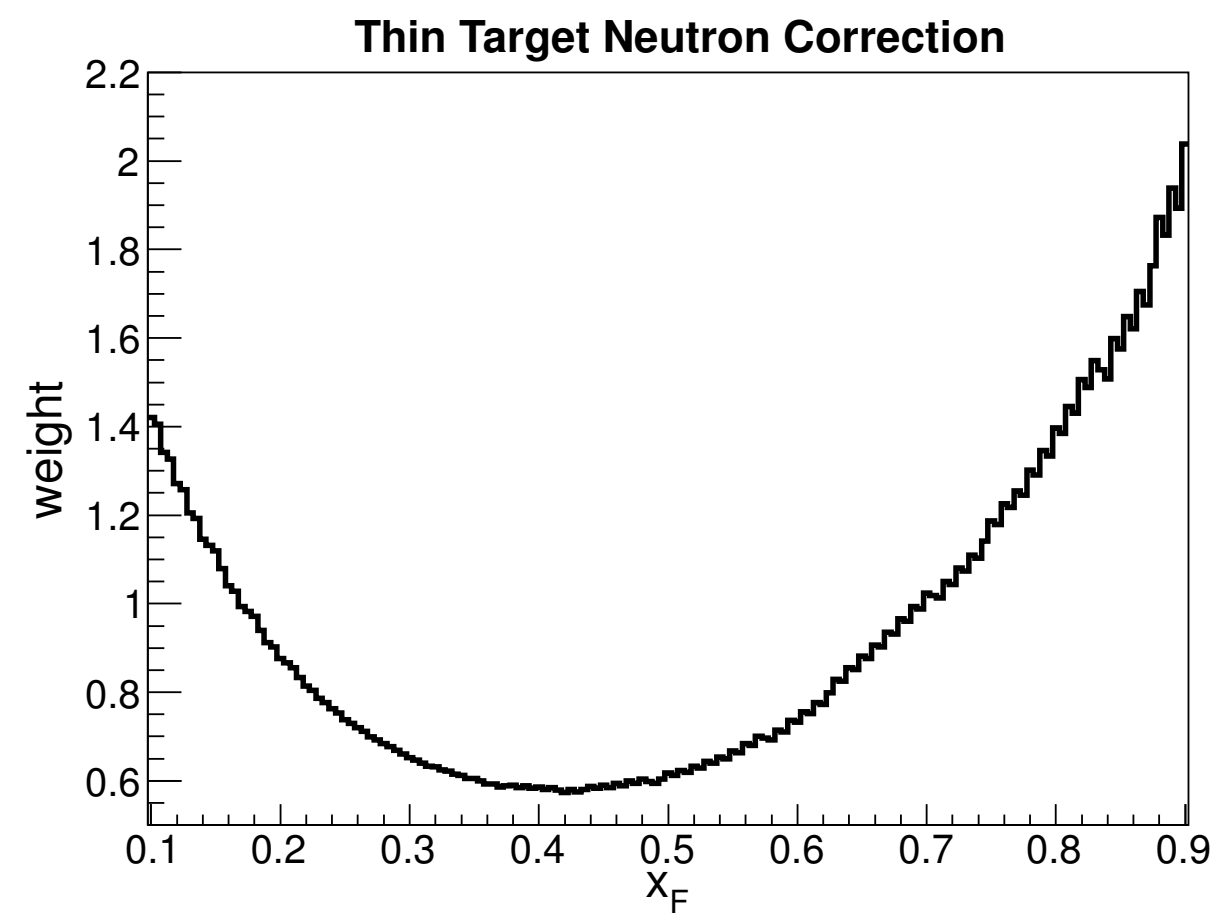

(b) neutron.

FIG. 4.39: Thin target weights for nucleon production. 
use of hadronic model spread as a procedure for these cases.

\subsubsection{Interactions of nucleons not covered by thin target data}

For interactions with nuclei other than carbon, we can apply the thin target carbon data from the previous section following the procedure described in Section 4.2.3. The material scaling adds the uncertainty listed in Table 4.1 to the data uncertainty for charged pions and kaons with $0<x_{F}<0.75$ and $p_{T}<1.5 \mathrm{GeV} / \mathrm{c}$. Another extension we use is to apply proton interaction data to the neutron interactions using the isospin symmetry principle (Section 4.2.3).

For any other nucleon interaction not covered yet, we use our best guess uncertainty based on data as was described in Section 4.2.4, i.e., $40 \%$ uncertainty split in four $x_{F}$ uncorrelated regions in $0-1(0-0.25,0.25-0.50,0.50-0.75$ and $0.75-1.0)$ per nucleon incident: \{proton, neutron $\}$ and per particle produced: $\left\{\pi^{+}, \pi^{-}, K^{+}\right.$, $K^{-}, K^{0}$, proton, neutron, any other particle\}. This gives 64 different uncertainty parameters, treated as uncorrelated. One thing that should be noted is that the quasi-elastic interaction correction falls into this category.

\subsubsection{Incident mesons and other interactions}

Currently, we are not applying any data corrections for interactions with incident mesons. In these cases, we use $40 \%$ uncertainty for each of the 4 uncorrelated $x_{F}$ regions $(0-0.25,0.25-0.50,0.50-0.75$ and $0.75-1.0)$. We do this for each different combination of incident meson: $\left\{\pi^{+}, \pi^{-}, K^{+}, K^{-}, K^{0}\right.$, any other meson $\}$ and produced particle: $\left\{\pi^{+}, \pi^{-}, K^{+}, K^{-}, K^{0}\right.$, proton, neutron, any other particle $\}$. This gives 144 uncorrelated uncertainty parameters.

When an interaction is not covered by any data and any of the above categories, 
a correlated $40 \%$ uncertainty is applied. Examples of these interactions are $p C \rightarrow$ $\bar{p} X$. As we see in the next chapters, the contribution from these "other" kind of interactions is very small. 


\subsection{Thin target inelastic and absorption cross- section datasets}

\subsubsection{Proton - carbon inelastic cross-section}

Figure 4.40 shows the data and MC inelastic cross-sections comparisons for proton and neutron incident on carbon. The datasets are Bellettini et al [64], NA61 for T2K [40], Denisov et al. [65], Carroll et al. [73], NA49 [66] and Roberts et

al. [74]. Some inconsistency in terminology in Denisov et al leads us to assume that the data provided in the paper is the absorption cross-section instead of the inelastic cross-section. For that reason, we subtract the quasi-elastic part using the Roberts et al calculation based the Glauber model. The neutron cross-section is placed as a reference. The data suggests that the inelastic cross-section has no energy dependence up to $300 \mathrm{GeV}$ incident energy.

The MC inelastic cross-sections are shown with black markers. They have been calculated by running high statistics thin-target simulations of proton incident with different energies and looking at interactions where no new particles (mesons) are created. The MC has a nonphysical behavior, increasing its value monotonically when the energy is increased. However, the MC absorption cross-section is flat at $243.24 \mathrm{mb}$.

\subsection{2 $\pi^{ \pm}$absorption cross-sections}

Figure 4.41 shows the absorption cross-section for charged pions incident in carbon and aluminum up to $70 \mathrm{GeV} / \mathrm{c}$ momentum. The datasets used are Cronin et al [75], Denisov et al [65], Allaby et al [76], Allardyce et al [77], Longo et al [78], Vlasov et al [79], Bobchenko et al [80], Carroll et al [73]. The data suggest that the absorption cross-sections decrease monotonically above $1 \mathrm{GeV} / \mathrm{c}$ incident momentum. 


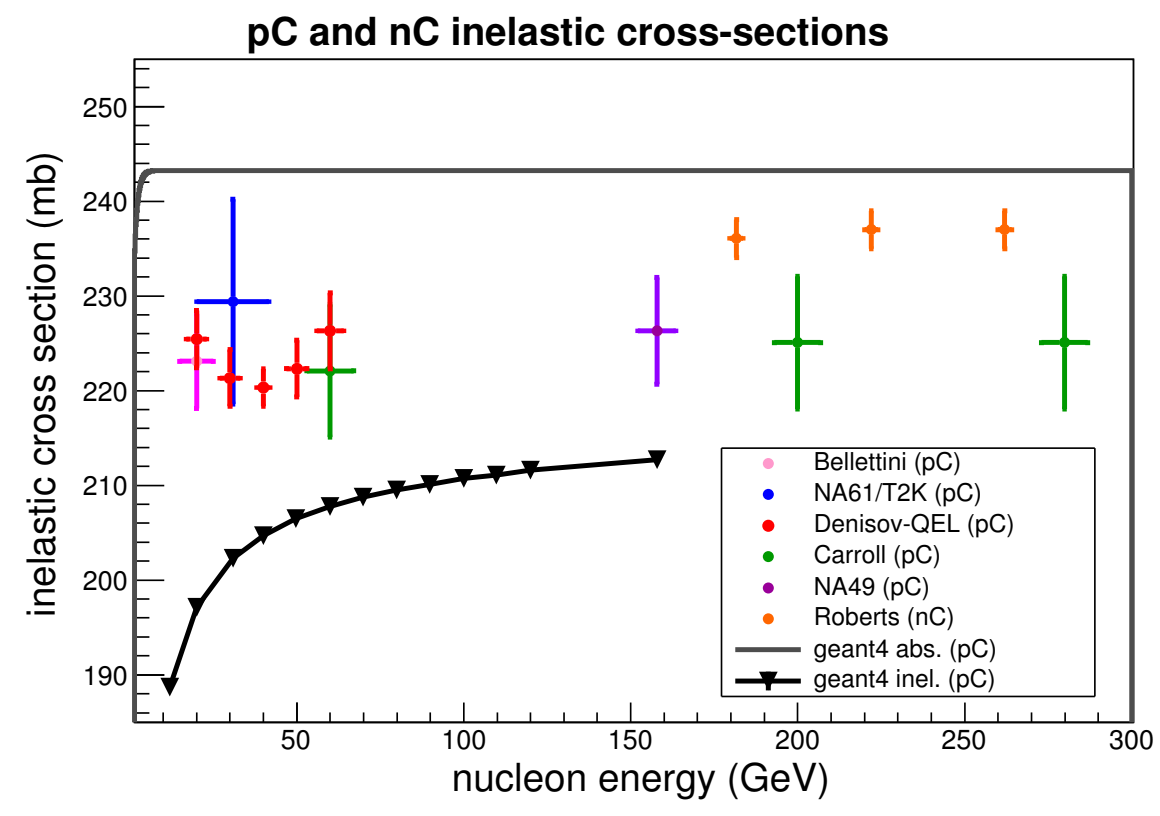

FIG. 4.40: Proton-carbon inelastic cross-sections.

The MC values are in blue (pion on carbon) and red (pion on aluminum).

Figures $4.42 \mathrm{a}$ and $4.42 \mathrm{~b}$ show the differences between data and MC absorption cross-sections. The data has a small disagreement with MC. For pion on carbon, most of the differences are less than $10 \mathrm{mb}$ and for pion on aluminum, less than $20 \mathrm{mb}$, except at low energies.

\subsection{3 $\quad K^{ \pm}$absorption cross-sections}

Figure 4.43 shows the absorption cross-section for charged kaon incident in carbon and aluminum up to $60 \mathrm{GeV} / \mathrm{c}$ momentum. The datasets used are Abrams et al. [81], Denisov et al. [65], Carroll et al. [73] and Allaby et al. [76]. There are few data points for $K^{+}$and no pattern has been found (c.f. Figure 4.43a ). For $K^{-}$, a slow cross-section decrease with energy can be seen in Figure 4.43b.

Figures 4.44 and 4.45 show the differences between data and MC absorption cross-sections for $K^{+}$and $K^{-}$on carbon and aluminum. The disagreement is larger 


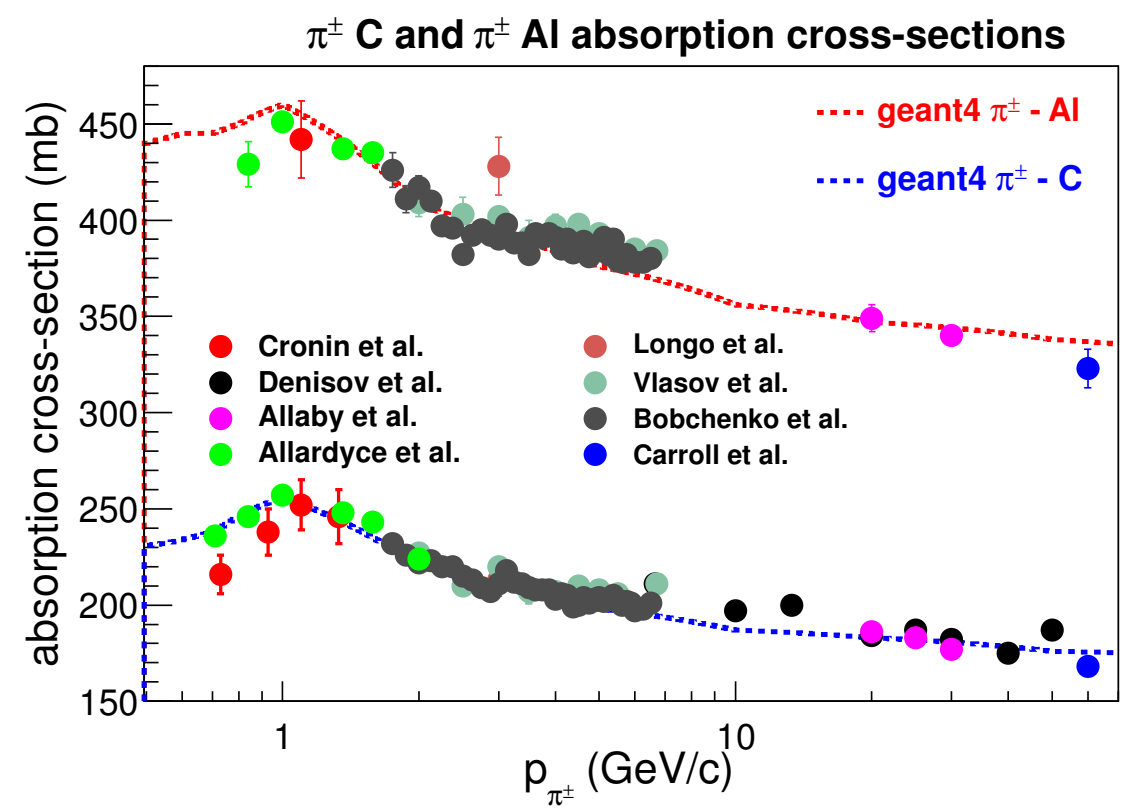

FIG. 4.41: $\pi \mathrm{C}$ and $\pi \mathrm{Al}$ data and MC absorption cross-section. Error bars on many of the points are too small to be seen.

than in charged pions, especially at low energy.

\subsubsection{Technical implementation of the absorption and in- elastic cross-section}

The correction is implemented as follows:

1. $\mathrm{pC}$ inelastic and absorption cross-sections:

- For the inelastic cross-sections (needed in cases when we use Equation 4.9), we implement the value given by NA49 data with its associated uncertainty $(226 \mathrm{mb} \pm 2.5 \%)$. For the absorption cross-section (needed for the proton attenuation correction in the target), we add the implemented inelastic crosssection and the quasi-elastic component, which has $40 \%$ uncertainty as described in Section 4.6.1. 


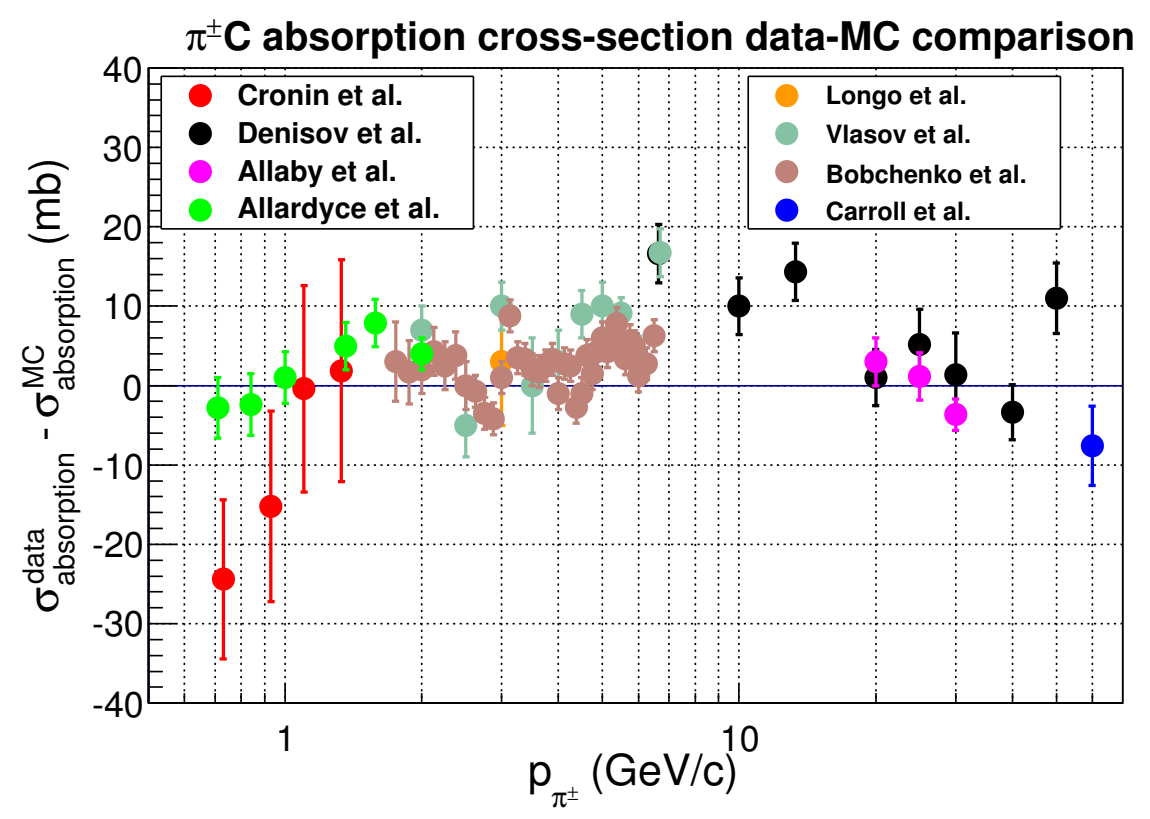

(a) $\pi \mathrm{C}$.

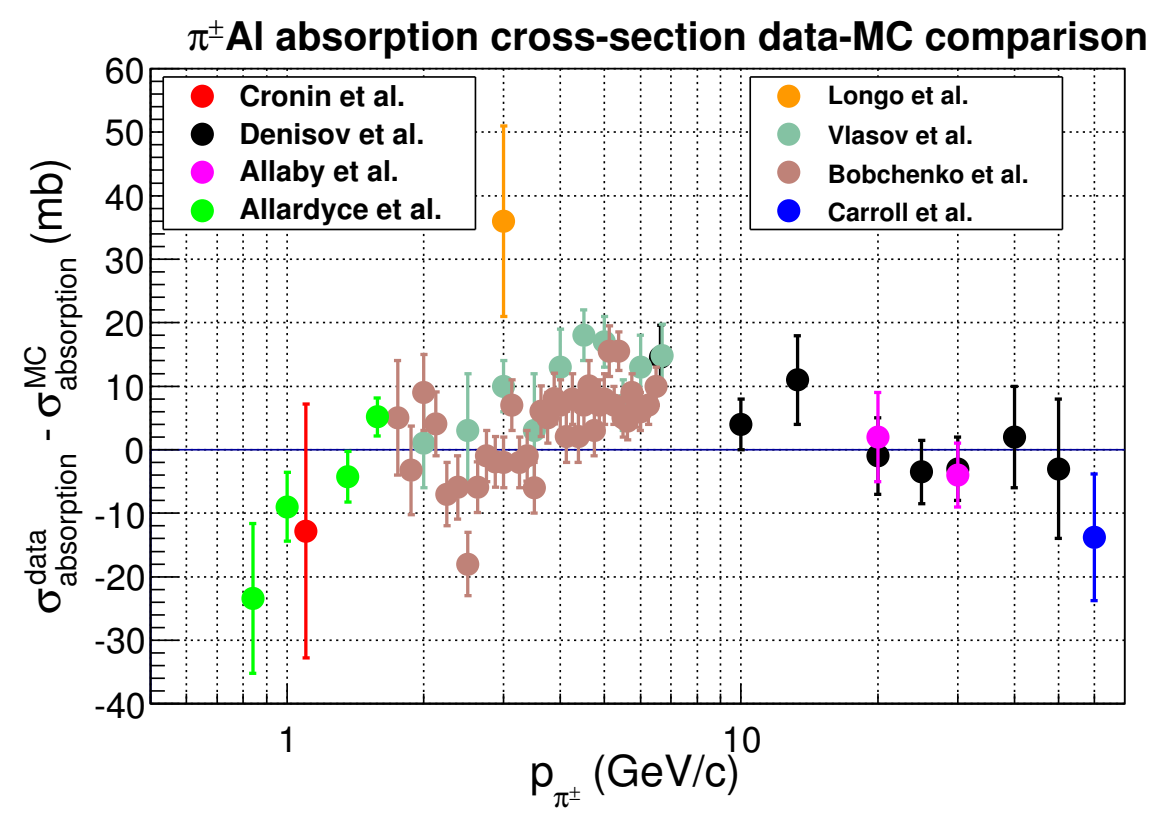

(b) $\pi \mathrm{Al}$

FIG. 4.42: $\pi$ Data - MC absorption cross-section data - MC comparisons. 


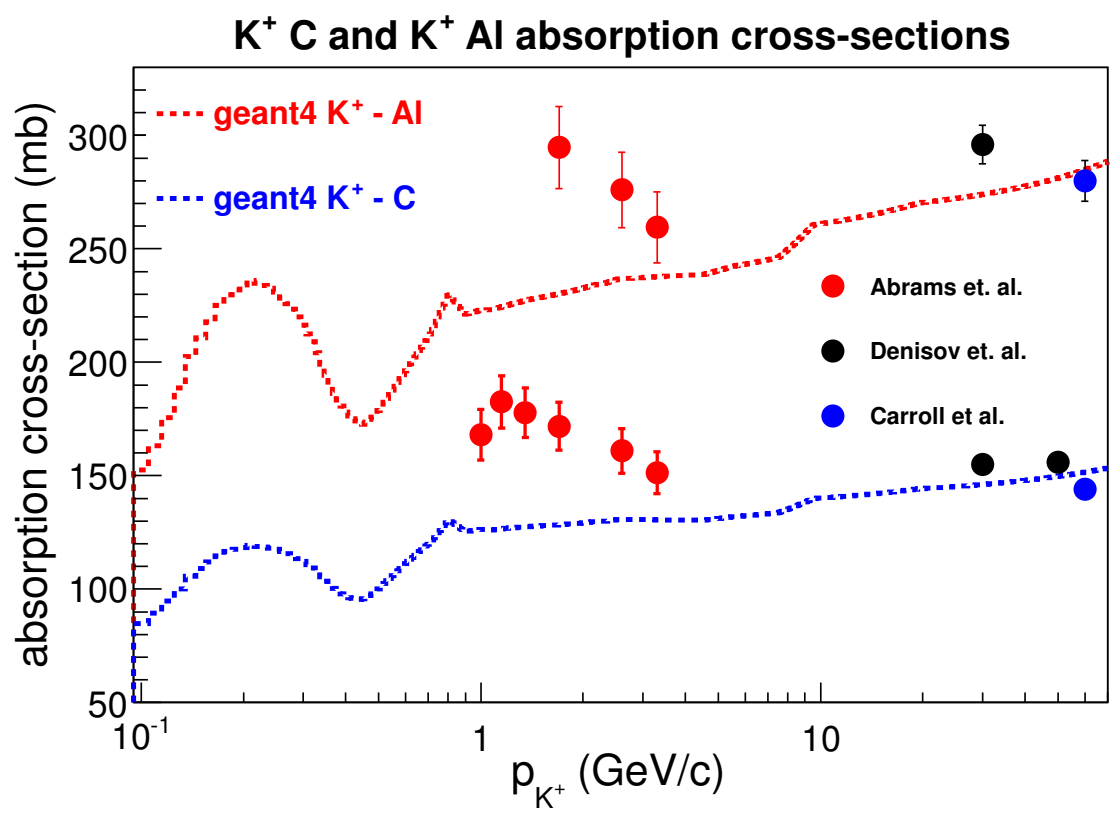

(a) $K^{+} \mathrm{C}$ and $K^{+} \mathrm{Al}$

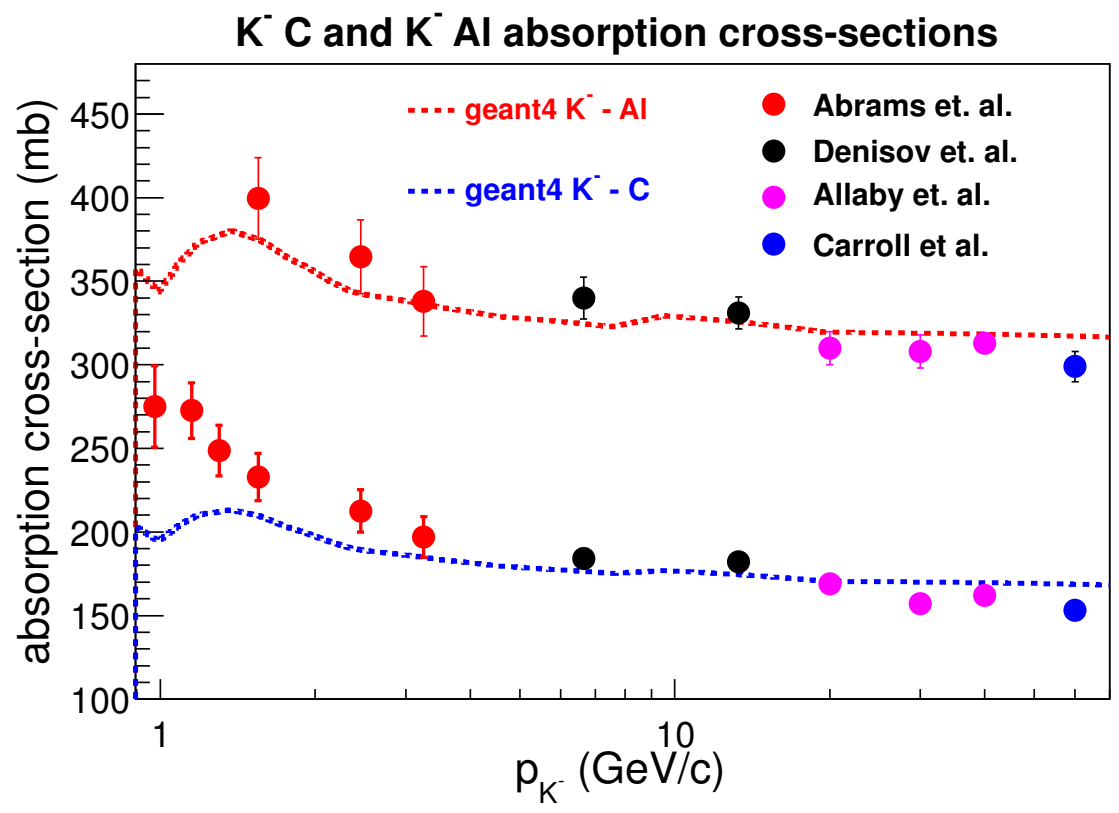

(b) $K^{-} \mathrm{C}$ and $K^{-} \mathrm{Al}$

FIG. 4.43: Charged kaon on $\mathrm{C}$ and $\mathrm{Al}$ data and MC absorption cross-section. Error bars on many of the points are too small to be seen. 


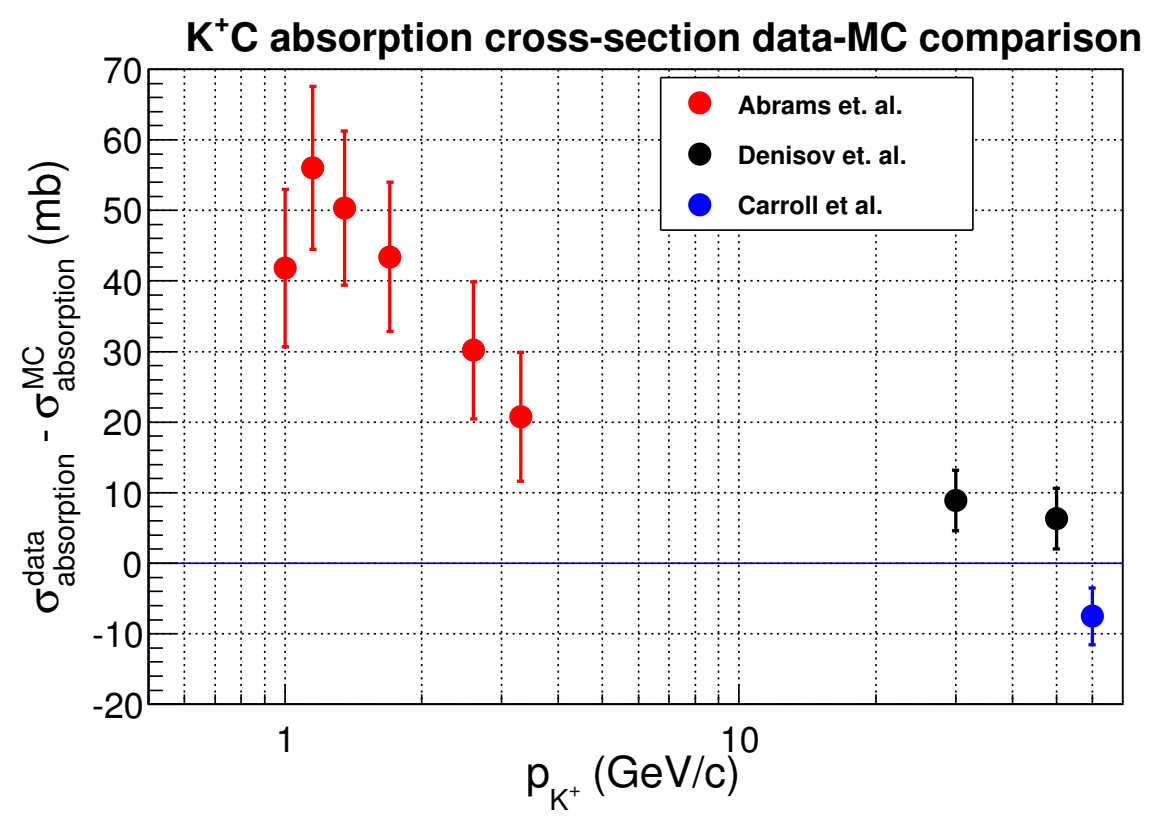

(a) $K^{+}$C.

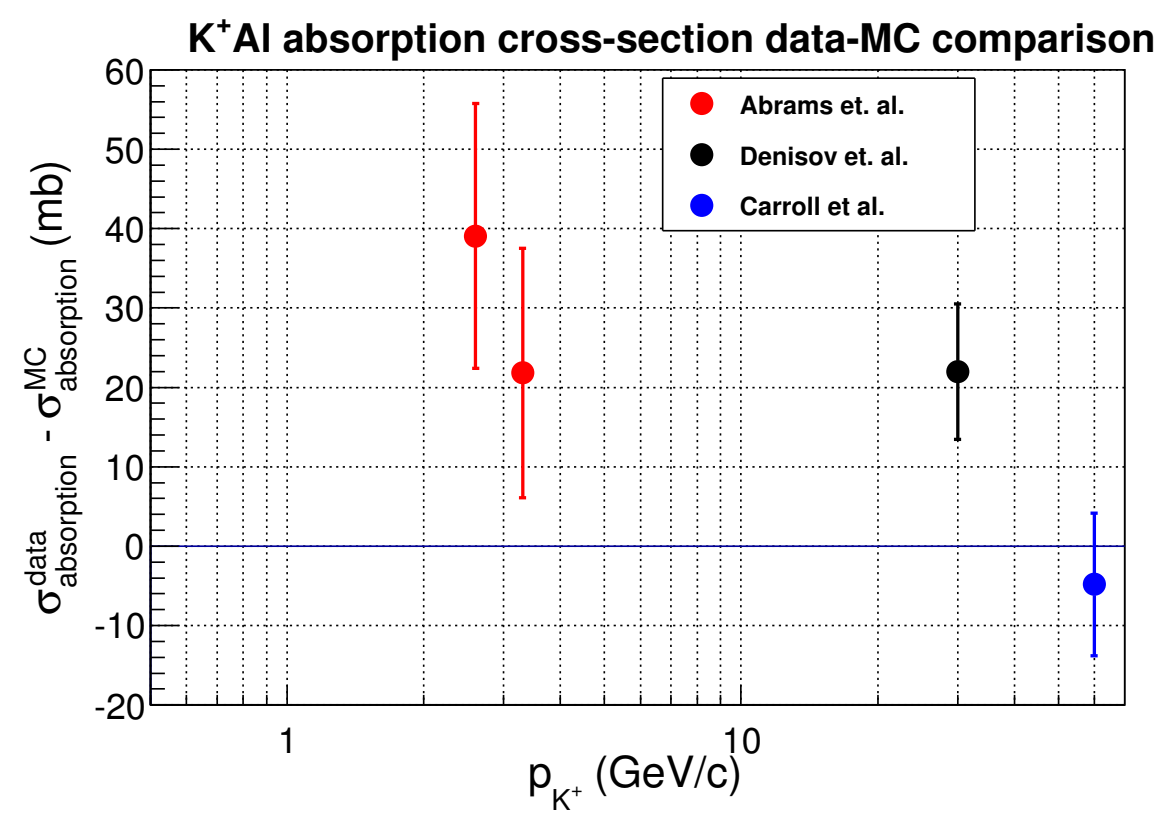

(b) $K^{+} \mathrm{Al}$.

FIG. 4.44: $K^{+}$data - MC absorption cross-section data - MC comparisons. 


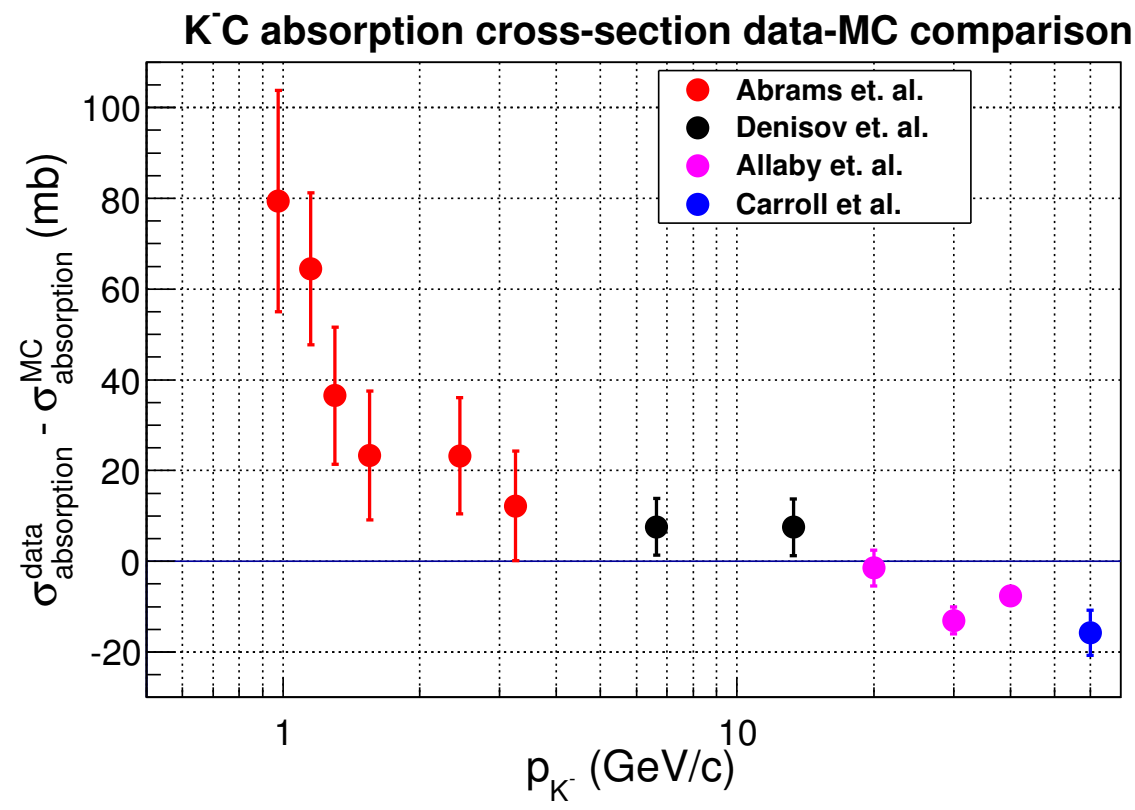

(a) $K^{-}$C.

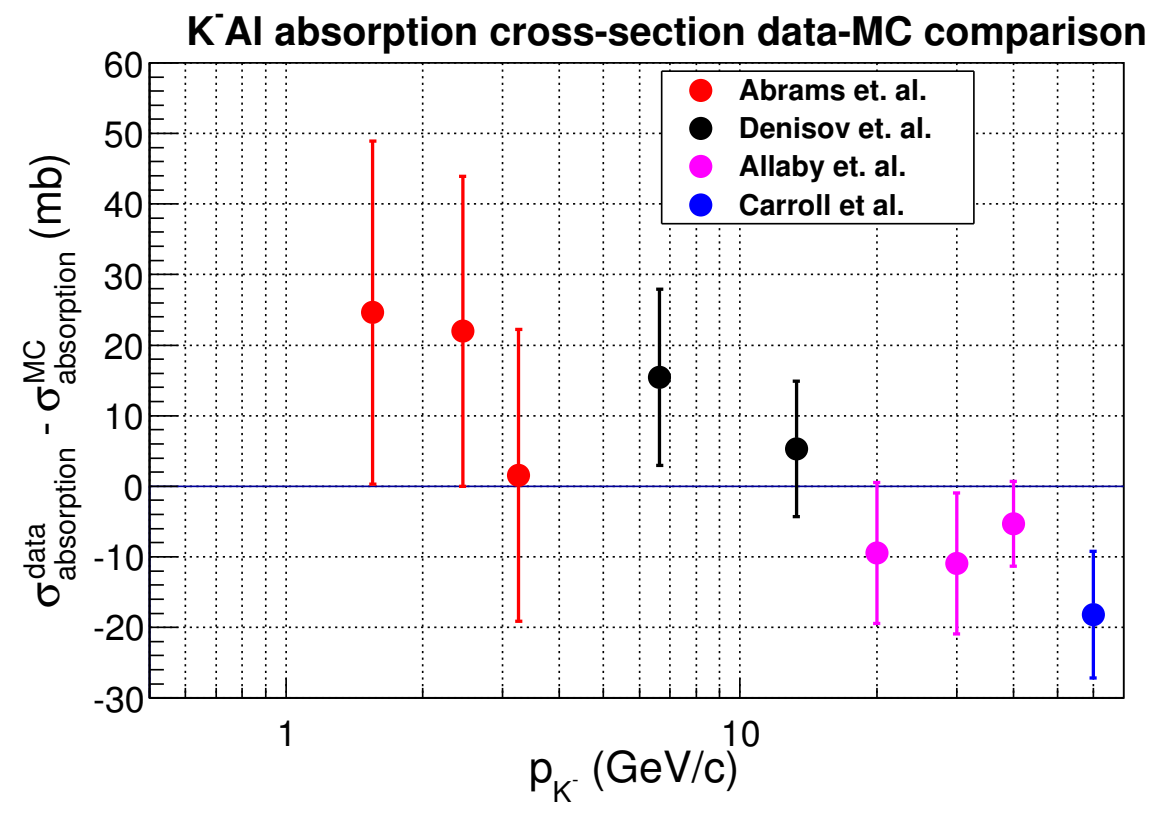

(b) $K^{-} \mathrm{Al}$.

FIG. 4.45: $K^{-}$data - MC absorption cross-section data - MC comparisons. 
- The MC, the calculated inelastic cross-section for the incident proton energy shown in Figure 4.40 is implemented and a linear interpolation is made for intermediate incident proton energies.

- We should note that this correction has a central value different than 1.

2. $\pi^{ \pm}$and $K^{ \pm}$absorption cross-section:

- The central value for this correction is equal 1 .

- For $\pi^{ \pm}$, we noted that almost all of the differences between the datasets and the $\mathrm{MC}$ are less than $\pm 5 \%$ of the $\mathrm{MC}$ value. Based on this, the uncertainty associated to $\pi^{ \pm} \mathrm{C}$ and $\pi^{ \pm} \mathrm{Al}$ are $10 \mathrm{mb}$ and $23.8 \mathrm{mb}$, respectively.

- For $K^{ \pm}$, the data and MC discrepancies are larger than $\pi^{ \pm}$, especially for low momentum $(<2 \mathrm{GeV} / \mathrm{c})$, and these discrepancies are different for $\mathrm{K}^{+}$ and $K^{-}$. Based on this, we implement uncertainties that cover almost all of the dataset - MC differences in Figures 4.44 and 4.45. Table 4.3 lists these values.

\section{Details about attenuation correction:}

- This correction is applied to all hadron neutrino parents, grandparents, and, when they exist, great-grandparents.

- For the target, the amount of carbon traversed longitudinally by the particles interacting or leaving the target is tabulated precisely. We add the material traversed only in the fins.

- The correction is also applied to others NuMI components: the horn inner conductor $(\mathrm{Al})$, the decay volume $(\mathrm{He})$ and decay pipe walls $(\mathrm{Fe})$.

- For nucleons, and in general any other particles, we assign the biggest uncertainty $\left(K^{+}\right.$on aluminum). 


\begin{tabular}{|l|c|}
\hline data & $\Delta \sigma(\mathrm{mb})$ \\
\hline$\pi$ on carbon & 10 \\
$\pi$ on aluminum & 23.8 \\
$K^{+}$on carbon $(P<2 \mathrm{GeV} / \mathrm{c})$ & 68 \\
$K^{-}$on carbon $(P<2 \mathrm{GeV} / \mathrm{c})$ & 80 \\
$K^{+}$on aluminum $(P<2 \mathrm{GeV} / \mathrm{c})$ & 83 \\
$K^{-}$on aluminum $(P<2 \mathrm{GeV} / \mathrm{c})$ & 49 \\
$K^{+}$on carbon $(P>2 \mathrm{GeV} / \mathrm{c})$ & 13 \\
$K^{-}$on carbon $(P>2 \mathrm{GeV} / \mathrm{c})$ & 20 \\
$K^{ \pm}$on aluminum $(P>2 \mathrm{GeV} / \mathrm{c})$ & 30.5 \\
\hline
\end{tabular}

TABLE 4.3: Summary of the meson absorption cross-section uncertainty implemented in our hadron production procedure.

A summary of the uncertainty per particle and material is listed in Table 4.3.

\subsection{PPFX}

Note: This is a technical section which may not interest some readers.

PPFX is the package we wrote to implement the hadron production corrections and propagate uncertainties described in the previous section. PPFX stands for Package to Predict the FluX and is an experiment-independent neutrino flux determination package for the NuMI beam that provides a correction for hadron production mismodeling ${ }^{8}$ using almost all relevant data. Currently, PPFX corrects the beamline simulated with g4numi using geant4_2.p03 and the FTFP_BERT hadronic model ${ }^{9}$. The inputs are dk2nu and dkmeta objects [82] for each neutrino event, and it returns a set of correction values to be used as weights to calculate the right

\footnotetext{
${ }^{8}$ The uncertainties associated with the focusing process have not been implemented yet and are currently handled inside the MINERvA framework.

${ }^{9}$ The prescription followed for the current model can be applied directly to extend to other hadronic models.
} 
neutrino yield. Internally, PPFX:

- Accounts for the attenuation of all particles passing through the relevant NuMI materials: the target and Budal monitors, the magnetic horn inner conductors, the decay pipe volume and the decay pipe walls.

- Implements energy-scaled thin-target data for incident particles in the energy range $12-120 \mathrm{GeV}$. All data is carefully interpolated to smooth transition between bins.

- Implements MIPP NuMI target data for pions and high energy kaons.

- When there are no data, PPFX attempts first to use theoretically guided extensions and when it is not possible, uses a data-driven best guess.

- Handles correlated and uncorrelated uncertainties using the multi-universe technique for uncertainty propagation.

Figure 4.46 shows a schematic view of how the main classes of PPFX work. They are described below:

\subsubsection{MakeReweight}

MakeReweight is a singleton class that controls the process. It has to be instantiated and initialized by the user selecting options that are entered in an xml file through the class member SetOptions. Three options are currently settable:

- The process mode. Two modes are implemented: MIPPNuMIOn and MIPPNuMIOff. When MIPPNuMIOn is selected, the code tries to use thick target data as a primary correction and thin target data for any interaction not yet covered. When MIPPNuMIOff is selected, the code does not use thick target at all and instead 


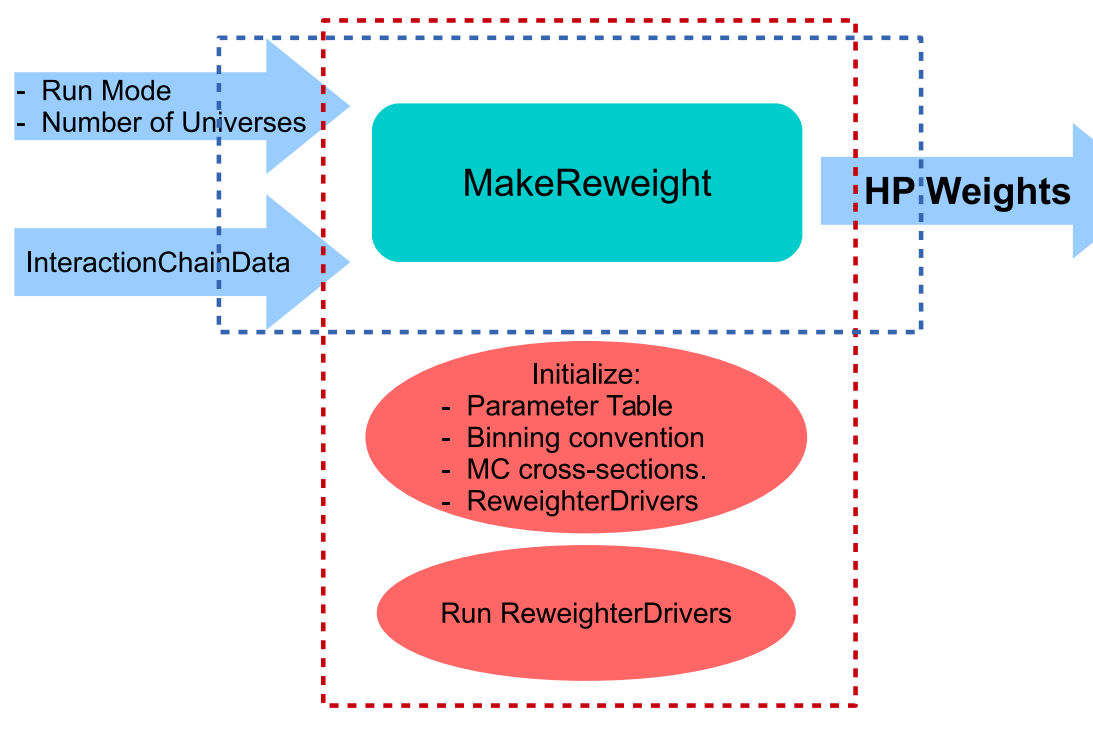

FIG. 4.46: PPFX flowchart.

applies a correction based on thin-target data for all interactions. This switching mechanism will be use for Gen2-thin and Gen2-thick flux versions as will be described in Section 5.3.

- The number of universes to be used in the process.

- The MIPP NuMI hadron production bin to bin correlation for systematic uncertainties. The default value is $+75 \%$.

MakeReweight is also in charge of initializing:

- The CentralValueAndUncertainties class that creates tables of central values and deviates per universe (it is called ParameterTable). This is done by reading the input data from an xml file (parameter file).

- The binning convention of the data input matching the parameter file convention. 
- A vector of ReweighterDriver's (where every entry corresponds to one universe and becomes its identification, ID) and a ReweighterDriver for the central value (which has an ID of -1).

Its member CalculateWeights constructs an InteractionChainData object using the dk2nu and dkmeta inputs, and passes them to every ReweighterDriver to make the calculations, and provide the user a vector of universe weights by calling the member GetWeights() and a central value weight by GetCVWeight().

\subsubsection{Data and MC Inputs}

The parameter file contains the input data from results of studies to make interpolations and correlations between bins. The implementation of every dataset has been presented and discussed during the MINERvA flux group meetings (which can be tracked looking at MINERvA doc-db ${ }^{10}$ ). For uncorrelated data, the central value and its uncertainty are provided as single values or a list of values. Correlated data requires a list of central values per bin, a list of statistical errors and a covariance matrix of the systematic errors. The CentralValueAndUncertainties class parses the parameter file and its member calculateParsForUniverse calculates a parameter table with all random data values per universe, where every universe has a unique and fixed random seed to make the process replicable and stable.

The MC values have been tabulated offline and, except for MIPP NuMI that enters as an $\mathrm{xml}$ file ${ }^{11}$, they are incorporated as ROOT histograms. Three classes store these values and provide them as requested by reweighters: AttenuationMC, ThinTargetMC and MIPPNumiMC.

\footnotetext{
${ }^{10}$ Some useful presentations are [83], [84] and [85] as well the implementation parts of Sections 4.4.1 to 4.7 .

${ }^{11}$ This is due to an early implementation before deciding to use ROOT files for MC inputs.
} 


\subsubsection{InteractionChainData}

The InteractionChainData class parses dk2nu and dkmeta objects and classifies the information in:

- A vector of InteractionData objects that contains information for an interaction (kinematics, identity of the projectile, target, and produced particles).

- A TargetData object with information for the hadron that exits the target: kinematics and identity of the hadron. This also includes its position index in the vector of InteractionChainData.

- A vector of ParticlesThroughVolumesData objects that stores the momentum and the amount of material crossed by the neutrino parent, grand-parent and when it exists, its great-grand-parent per volume.

\subsubsection{ReweightDriver}

The ReweightDriver class is in charge of initializing the specialized reweighters for specific interactions and using them to calculate hadron production weights. A reweighter makes the task for one universe, or for the central value, receiving the corresponding ParameterTable and the InteractionChainData from the MakeReweight class. The member CalculateWeights selects the appropriate reweighter and book a history of the interactions already corrected to avoid duplications.

\subsubsection{Reweighters}

These classes are responsible for the hard work (i.e. calculating the weights). Those are inherited from one of the two following abstract base classes: 
- IInteractionChainReweighting. The interface for classes that correct a chain of interactions given an InteractionChainData. Two members have to be implemented by reweighters inherited by this class: canReweight and calculateWeight. The former looks at the InteractionChainData input and identifies those interactions that can be corrected as part of a chain and returns a vector indicating which elements will be assigned a weight by calculateWeight.

- IInteractionReweighting. The interface for classes that corrects one interaction given by InteractionData. As before, canReweight and calculateWeight have to be implemented by every reweighter.

The reweighters associated with the attenuation correction are special because even when they are inherited from IInteractionChainReweighting, they are not specialized in correcting hadron production interactions and they use the InteractionChainData to get the vector of ParticlesThroughVolumesData.

The complete list of reweighters is given in Table 4.4.

X 


\begin{tabular}{|l|l|l|}
\hline Type & Name & Sections \\
\hline Thick target & MIPPNumiPionYieldsReweighter & 4.4 .1 \\
& MIPPNumiKaonYieldsReweighter & 4.4 .2 \\
\hline Thin target & ThinTargetpCPionReweighter & 4.5 .4 \\
& ThinTargetpCKaonReweighter & 4.5 .5 \\
& ThinTargetpCNucleonReweighter & 4.5 .6 \\
& ThinTargetnCPionReweighter & 4.6 .1 \\
& ThinTargetnucleonAReweighter & 4.6 .1 \\
& ThinTargetMesonIncidentReweighter & 4.6 .2 \\
& OtherReweighter & 4.6 .2 \\
\hline Attenuation & TargetAttenuationReweighter & $4.7 .1-4.7 .3$ \\
& AbsorptionICReweighter & \\
& AbsorptionDVOLReweighter & \\
& AbsorptionDPIPReweighter & NucleonAbsorptionOutOfTargetReweighter \\
& OtherAbsorptionOutOfTargetReweighter & \\
\hline
\end{tabular}

TABLE 4.4: PPFX reweighters. 


\section{CHAPTER 5}

\section{A priori MINERvA Low Energy Beam results}

This chapter is dedicated to presenting the a priori LE flux results at MINERvA. As was mentioned in Section 3.5, the flux prediction has been permanently updated to add new hadron production data, to increase the accuracy of the NuMI simulation's geometry, and to improve the treatment of the flux systematics.

Historically, we have had two NuMI a priori flux predictions called Generation 0 and Generation 1 that were used in published MINERvA papers. Table 5.1 has a list of the MINERvA papers with their flux versions. The most complete version is Generation 2 which includes the hadron production data constraints described throughout Sections 4.4 to 4.7 .

There are some commonalities between flux generations:

- The use of the multi-universe technique (see Section 4.2.5) to propagate the hadron production and focusing uncertainties.

- The use of G4numi as MC flux simulation and FTFP_BERT as the hadronic physics model. 
Additionally, it is worthwhile to emphasis that the MINERvA flux results are calculated integrating over the MINERvA fiducial volume defined as the region inside of an hexagon with $86 \mathrm{~cm}$ apothem transverse area to the beam axis and within a $126.7 \mathrm{~cm}$ region along $\mathrm{z}$ axis corresponding to the "downstream" portion of tracker region. Our procedure is the following:

- We select neutrino inclusive charged-current interactions on carbon produced in the fiducial volume. The selection is made using the MC truth information.

- Based on the neutrino ancestry record, we apply hadron production corrections and propagate the uncertainties associated to the hadron production and the focusing system. For Generation 0 and Generation 1, this is made using a code inside the MINERvA offline computational framework. For Generation 2, we use PPFX as an external package to the MINERvA framework .

- The event rate per neutrino type is then divided by the corresponding GENIE cross-section and the number of carbon atoms in the fiducial volume.

This chapter is structured into 4 sections. In the first two sections, we will explain the procedure and results for Generation 0 and Generation 1. We present the Generation 2 results in Section 5.3 while offering an analysis of the contribution of every PPFX reweighter. In the final section, we discuss the implications of the Generation 2 results.

\subsection{MINERvA flux Generation 0}

Generation 0 has been calculated with the following components:

- Hadron production central value. We only used thin target data to correct interactions in the target. The energy scaling described in Section 4.2 .2 is also used. We applied: 
- NA49 (Section 4.5.1) and Barton (Section 4.5.2) charged pion production on proton - carbon interactions (Figure 4.25).

- NA49 charged kaon production on proton - carbon interactions (Figure 4.29).

- NA49 proton production on proton - carbon interactions (Figure 4.36).

- Attenuation of the primary protons that interact in the target were applied using NA49 inelastic cross-section (Sections 4.2.1 and 4.7.1).

- Hadron production uncertainties. The uncertainties applied were:

- The statistical and systematic uncertainties added in quadrature of the NA49 pion production on proton - carbon interactions to the primary proton interactions producing pions in the target (Figure 4.27 and Table 4.2).

- The model spread between geant4 hadronic models was applied to the rest of the interactions 4.2 .4 .

\section{- Focusing:}

- We applied the focusing uncertainties inherited from MINOS [25]: transverse horn offset (Section 2.5.1), baffle scraping (Section 2.5.2), POT counting (Section 2.5.3), horn current magnitude (Section 2.5.4), current distribution in the horn inner conductors (explain in Section 2.5.4) and the longitudinal target offset (Section 2.5.6).

- The flux is simulated using the nominal target positions (Table 2.3), i.e., it was before implementation of the longitudinal target position survey described in Section 2.5.6.

Figure 5.1a shows the Generation $0 \nu_{\mu}$ and $\bar{\nu}_{\mu}$ fluxes from LE010z185i and LE010z-185i respectively before the HP correction and after applying the correction. The size and energy dependence of the HP correction can be seen in the Figure 5.1b. 


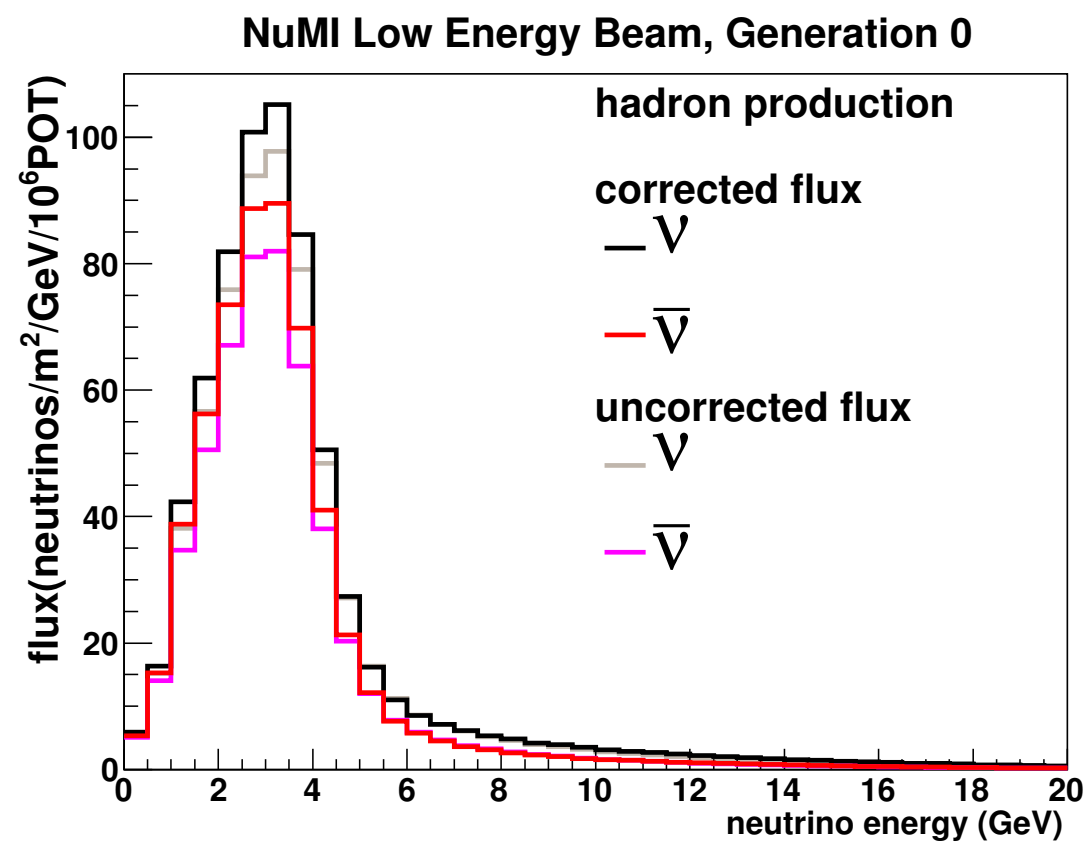

(a) Flux spectra.

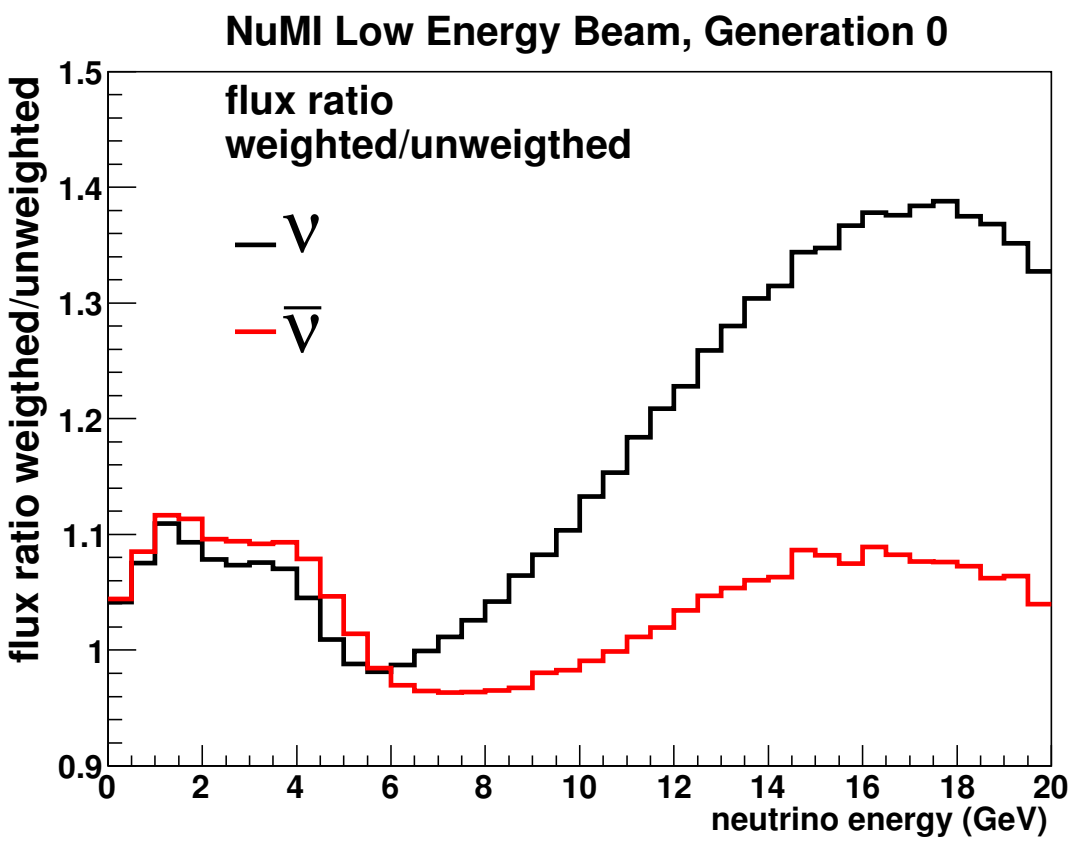

(b) Ratio weighted / unweighted.

FIG. 5.1: $\nu_{\mu}$ and $\bar{\nu}_{\mu}$ Generation 0 flux. 


\subsection{MINERvA flux Generation 1}

Generation 1 has been calculated considering:

- Hadron production Central value. In addition to the datasets included in Generation 0, we used:

- MIPP $K / \pi$ yield ratios to extend the NA49 data coverage (Figure 4.30).

- The HP correction extended to all materials (not only to the target) using the same weights calculated for carbon. This was the first effort to extend the data coverage to other materials, but it was without any scaling studies yet.

- A bug fixed on our computation of the MC invariant cross-section for $\pi^{ \pm}$. The Geant4 hadronic models produce explicitly $\eta, \eta^{\prime}, \Sigma^{0}$ and $\pi^{0}$ when simulating the hadronic interactions. Hadron production experiments do not explicitly measure $\eta$ and $\eta^{\prime}$ and they can decay into charged pions. Then, cases in which there is a charged pion from an $\eta\left(\eta^{\prime}\right)$ decay has to be accounted when calculating the invariant cross sections.

- Hadron production Uncertainties. There was no change in the hadron production uncertainties.

- Focusing. There was no change in the focusing uncertainties.

Figure 5.2a shows the Generation $1 \nu_{\mu}$ and $\nu_{e}$ fluxes from LE010z185i, respectively, and $\bar{\nu}_{\mu}$ flux from LE010z-185i before the HP correction and after applying the correction. The size and energy dependence of the HP correction can be seen in $5.2 \mathrm{~b}$. The differences with Generation 0 around the focusing peak come mainly from the fix of the $\eta\left(\eta^{\prime}\right)$ bug. 


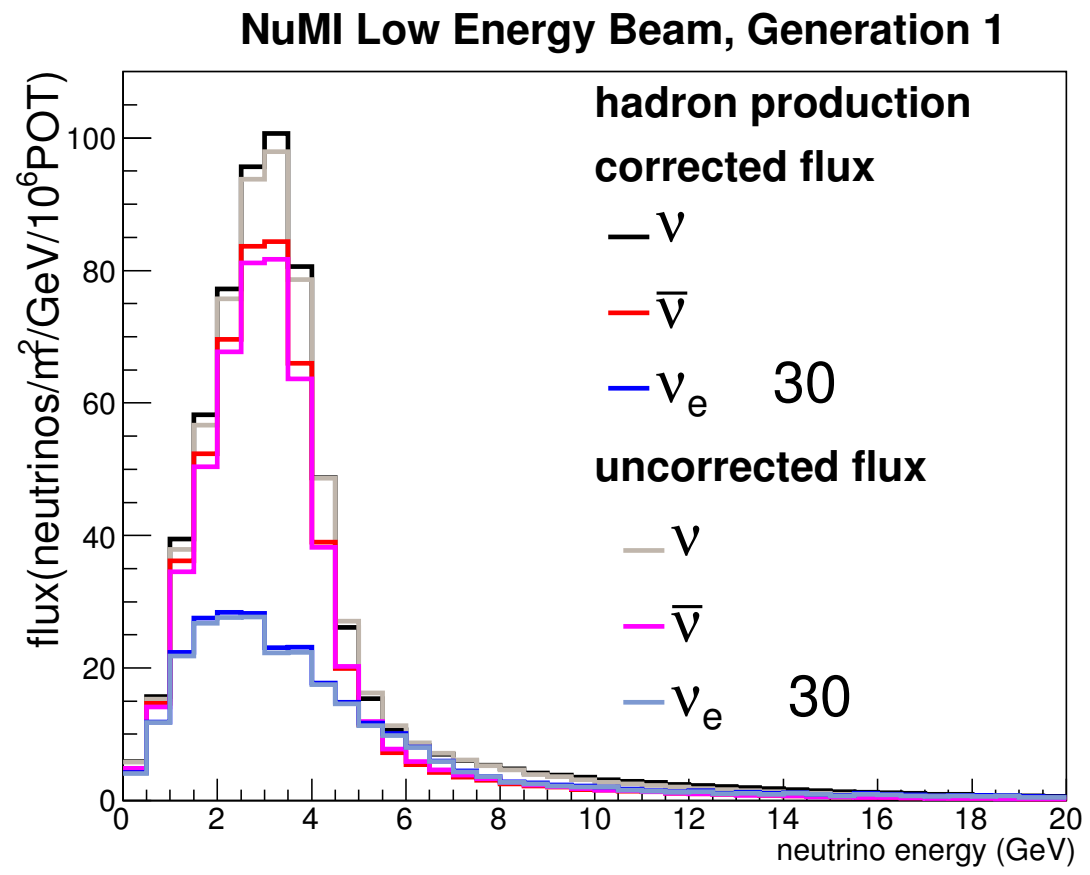

(a) Flux spectra.

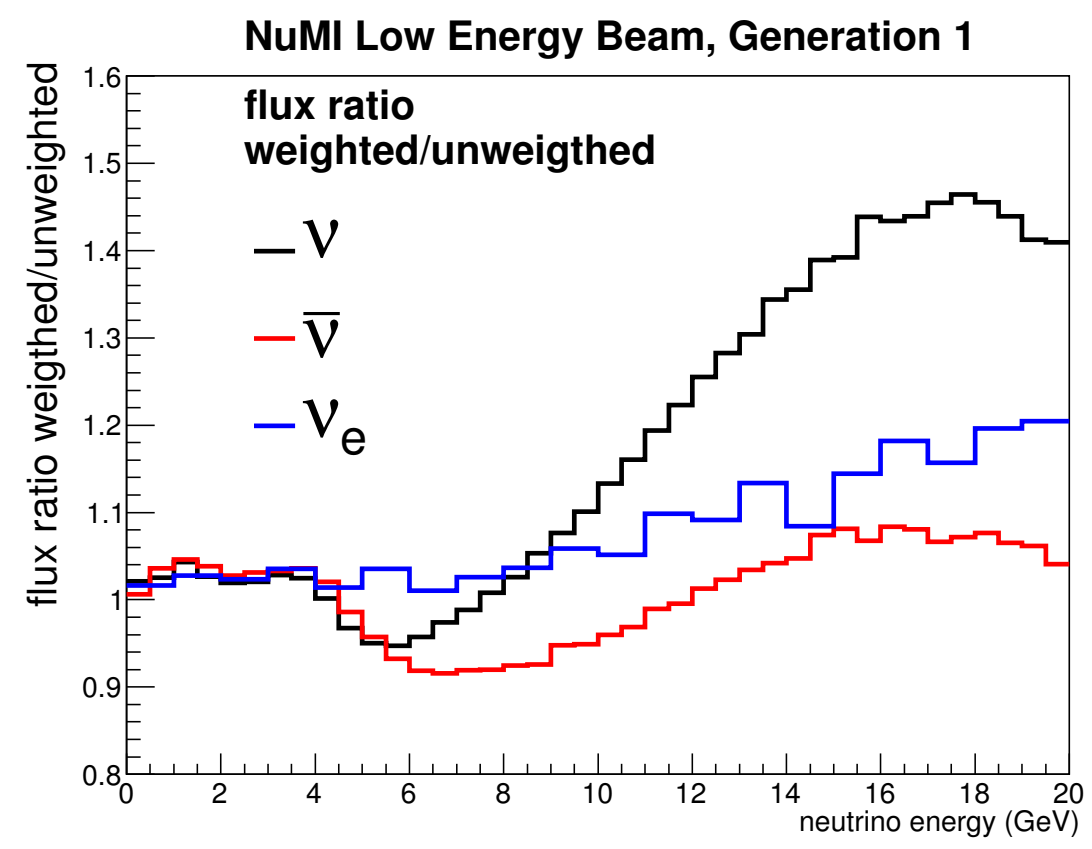

(b) Ratio weighted / unweighted.

FIG. 5.2: Generation 1 flux. 
Figure 5.3 shows the $\nu_{\mu}$ and $\nu_{e}$ flux fractional uncertainties $\left(\bar{\nu}_{\mu}\right.$ is similar to $\nu_{\mu}$ ). As was explained before, the uncertainties did not change when we moved from Generation 0 to Generation 1. The uncertainty from the model spread ("no data constraint" in the plot) dominates the $\nu_{\mu}$ total uncertainty, except around the focusing peak where NA49 data are used for most of the interactions that contribute to that neutrino energy region (red line). For the $\nu_{e}$ flux, the large contribution of highly energetic kaon neutrino parents makes the model spread (and the total uncertainty) larger than for $\nu_{\mu}$.

Table 5.1 contains a complete list of the MINERvA papers currently published. As was mentioned before, they will need to be updated with Generation 2 flux results (see Chapter 6).

\begin{tabular}{|l|l|}
\hline Flux & Analysis \\
\hline Generation 0 & $\begin{array}{l}\nu_{\mu} \text { Charged Current Quasi-elastic Scattering [86] } \\
\bar{\nu}_{\mu} \text { Charged Current Quasi-elastic Scattering [87] }\end{array}$ \\
\hline Generation 1 & $\begin{array}{l}\text { Charged Current Scattering Target Ratios [88] } \\
\text { Coherent } \pi \text { Production [10] } \\
\text { Muon }+ \text { Proton Production in } \nu_{\mu} \text { Scattering [89] } \\
\text { Charged Current } \pi^{0} \text { Production in } \bar{\nu}_{\mu} \text { Scattering [90] } \\
\text { Charged pion production in } \nu_{\mu} \text { interactions [91] }\end{array}$ \\
\hline
\end{tabular}

TABLE 5.1: Flux used in the current MINERvA publications.

\subsection{MINERvA flux Generation 2}

In May of 2014, we started working on a new flux version - Generation 2 motivated by:

\section{- Hadron Production:}




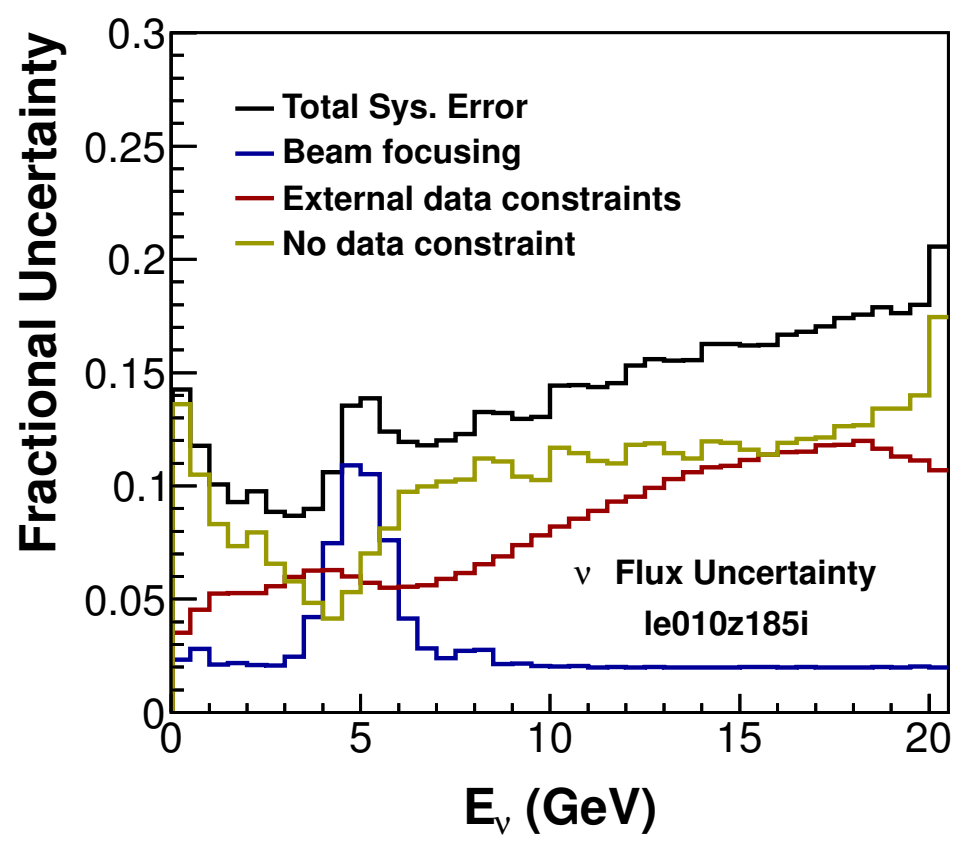

(a) $\nu_{\mu}$.

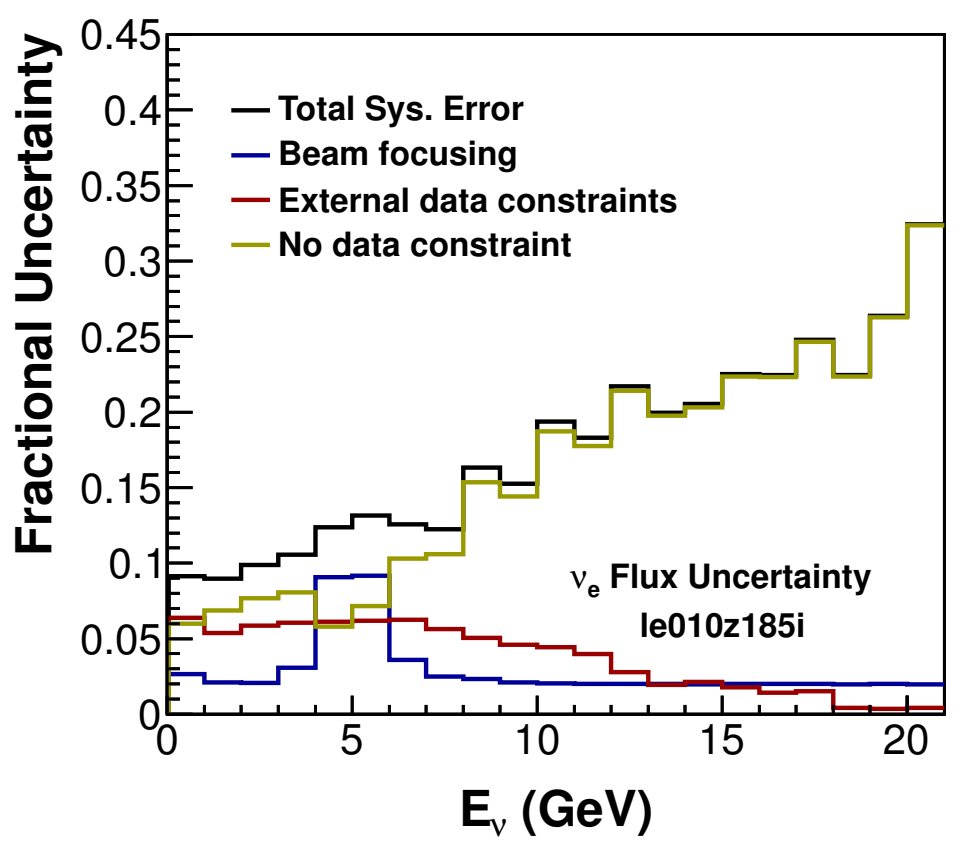

(b) $\nu_{e}$.

FIG. 5.3: Generation 0 and Generation 1 flux fractional uncertainties for $\nu_{\mu}$ and $\nu_{e}$ from LE010z185i. 
- Thick target data was published by the MIPP experiment using a LE NuMI target (see Section 4.4.1).

- The need to re-evaluate the flux uncertainties and gain a deeper understanding of them by replacing the model spread with direct data or physics motivated data extensions for the corrections (Section 4.2.4).

- The inclusion of particle absorption in the beamline volumes (Section 4.2.1) beyond primary proton in the target.

\section{- Improvements in the geometry simulation of NuMI that implement:}

- accurate target positions (Section 2.5.6).

- a water layer around the horn inner conductors, and other previously missing materials (Section 2.5.7).

- an improved horn geometry model (Section 2.5.5).

In this section, we present the Generation 2 Low Energy Beam flux results for $\nu_{\mu}$ in minerva13, $\bar{\nu}_{\mu}$ in minerva5 and $\nu_{e}$ in minerva13. See Section 2.5.6 for the playlist definition.

The implementation of thin and thick target data in PPFX, following the procedure in Section 4.2, gives us the opportunity to have two flux versions that are called Generation2 - thin (Gen2-thin) and Generation2 - thick (Gen2-thick).

Gen2-thin uses primarily thin target data to correct hadronic interactions (see Section 4.5). The first attempt is to use data directly and then, when that is not possible, we look to extend the data (i.e. for $\mathrm{pA}$ and $\mathrm{nA}$ interactions, see Section 4.2.4). For the remaining interactions $(\pi A \rightarrow \pi A$, etc.), we make an educated guess (see Section 4.2.4). The attenuation of the particle beams is corrected for any hadron passing through the relevant NuMI volumes: TGT, IC, DVOL and DPIP (see Section 4.7). 
Figures 5.4a and 5.4b show the average number of hadronic interactions per $\nu_{\mu}$ and $\nu_{e}$ in MINERvA that are handled by each reweighter in Gen2-thin (the $\bar{\nu}_{\mu}$ plots are not shown here since are similar to the $\nu_{\mu}$ ones). The black lines represent the total average. Except for low neutrino energies $(<1 \mathrm{GeV})$, these values are around 1.4, meaning that a large fraction of these interactions happen inside of the target as we can see when we compare this with the interactions covered by hadrons that exit the target (Figures 4.12 and 4.13).

Every line represents the coverage of one PPFX reweighter (see Table 4.3):

- MIPP NuMI $\pi$ and K production are shown by solid and dashed red lines respectively .

- Pion, kaon and nucleon production in proton - carbon interactions using thin target data are shown by solid red, dashed red and dashed orange lines respectively.

- Pion production in neutron carbon interactions constraint with data using isoscalar principles are shown by solid orange lines.

- Meson incident interactions are shown by solid brown lines.

- Nucleon incident interactions not covered directly by any data (it is called nucleonA in the plots) are shown by dashed brown lines.

- Others includes the remaining interactions not covered by prior considerations, for example, $p C \rightarrow \pi^{+} X$ at $x_{F}>0.5$ and $p_{T}>0.5$ that are not covered by neither NA49 nor Barton.

The dominant corrections handled by PPFX for $\nu_{\mu}\left(\nu_{e}\right)$ are for pion production from proton carbon interactions up to $20 \mathrm{GeV}(8 \mathrm{GeV})$ after which the kaon production correction becomes relevant at high energies in accordance with their ancestry 
(see Figure 2.16a ). The coverage around the focusing peak is almost complete (compare with the Figure $2.17^{1}$ ).

The other significant component is nucleon-A, especially around the transition between pion and kaon production. This is because only a small region with $x_{F}>0.5$ is covered by Barton. The nucleon interactions, nucleon production and meson incident corrections have higher values at low energies because they are composed by secondary and tertiary hadron production.

Gen2-thick uses thick target data primarily, and then, for the interactions still not covered, uses thin target data and an educated guess. The beam particle attenuation is corrected for any hadron passing through the IC, DVOL and DPIP. For TGT, only the primary proton beam attenuation (in combination with thick target data) is corrected (see Section 4.2.2).

Figures $5.5 \mathrm{a}$ and $5.5 \mathrm{~b}$ show the effects of thick target data on the average number of interactions covered by every reweighter in Gen2-thick. The data for MIPP NuMI pion and kaon cover many of these interactions, especially around the focusing peak. Thin target pion production becomes relevant only for low energies due to the MIPP acceptance (see 4.15 for an example). The contribution from nucleon-A also decreases, particularly due to the coverage of secondary protons reinteracting in the target to make pions. The small bump for thin target kaon production around 8-19 GeV comes from kaons below $20 \mathrm{GeV}$ covered by NA49 $\left(x_{F}<0.2 \mathrm{GeV}\right)$.

We split nucleon-A into more categories in order to understand which corrections are being handled for this reweighter: Figure 5.6a for Gen2-thin and 5.6b for Gen2-thick. Some of the characteristics are:

- Pion and kaon production from nucleon interactions (solid blue line) using the

\footnotetext{
${ }^{1}$ Figure 2.17 shows the average number of interactions integrated over the neutrino energy in 0-20 GeV but it is a good approximation for the focusing peak.
} 


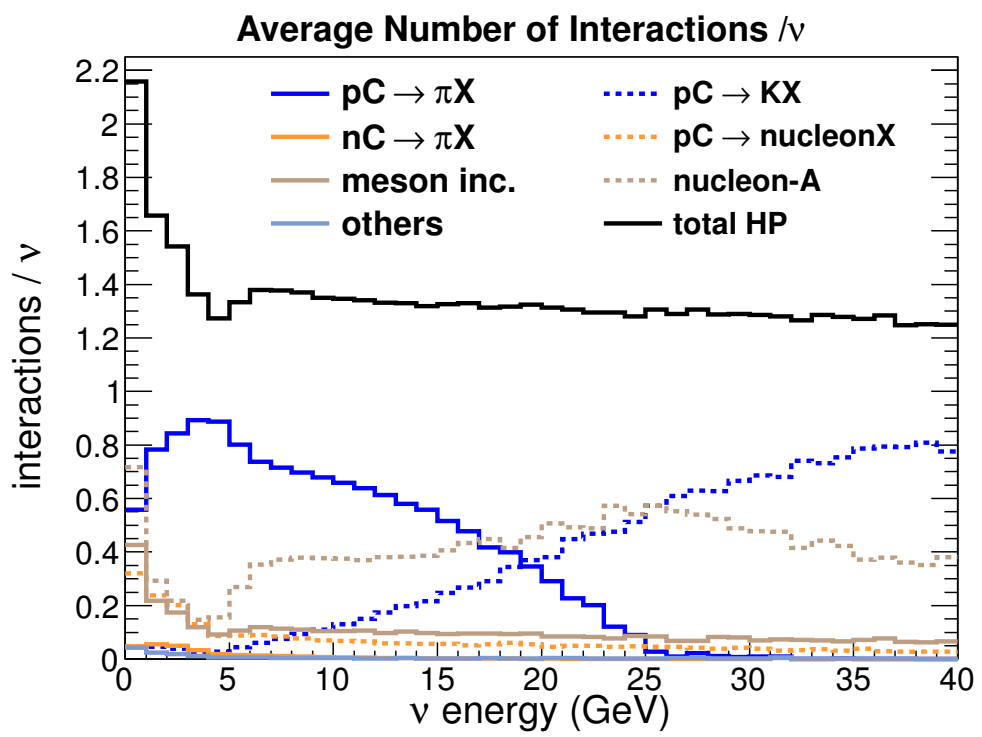

(a) $\nu_{\mu}$.

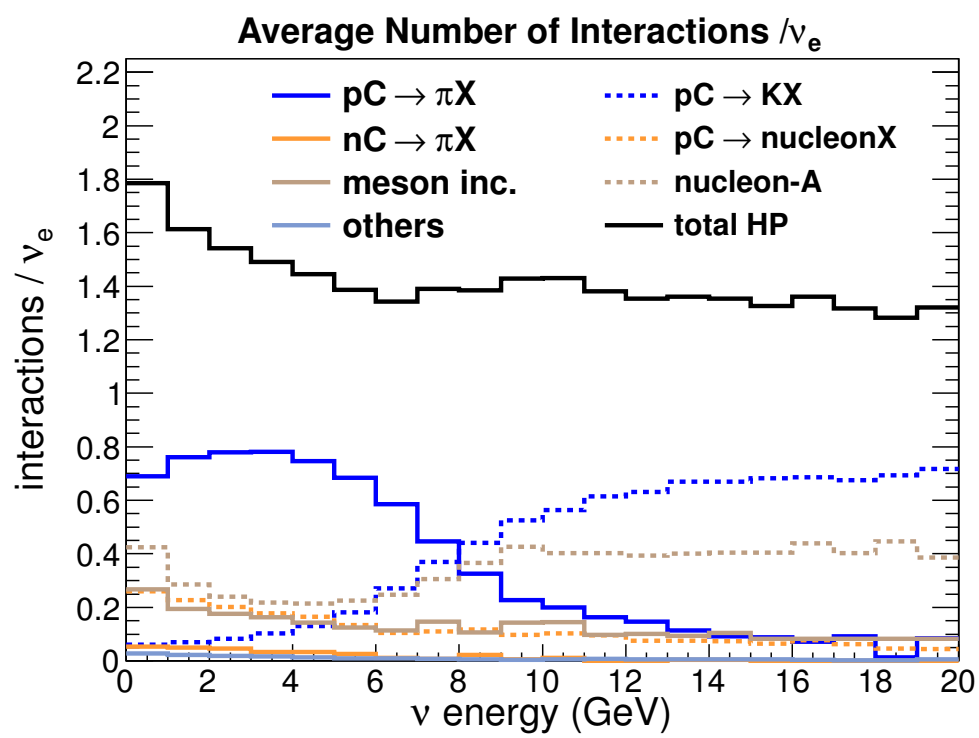

(b) $\nu_{e}$.

FIG. 5.4: Gen2-thin average number of hadronic interactions per neutrino minerva13. 


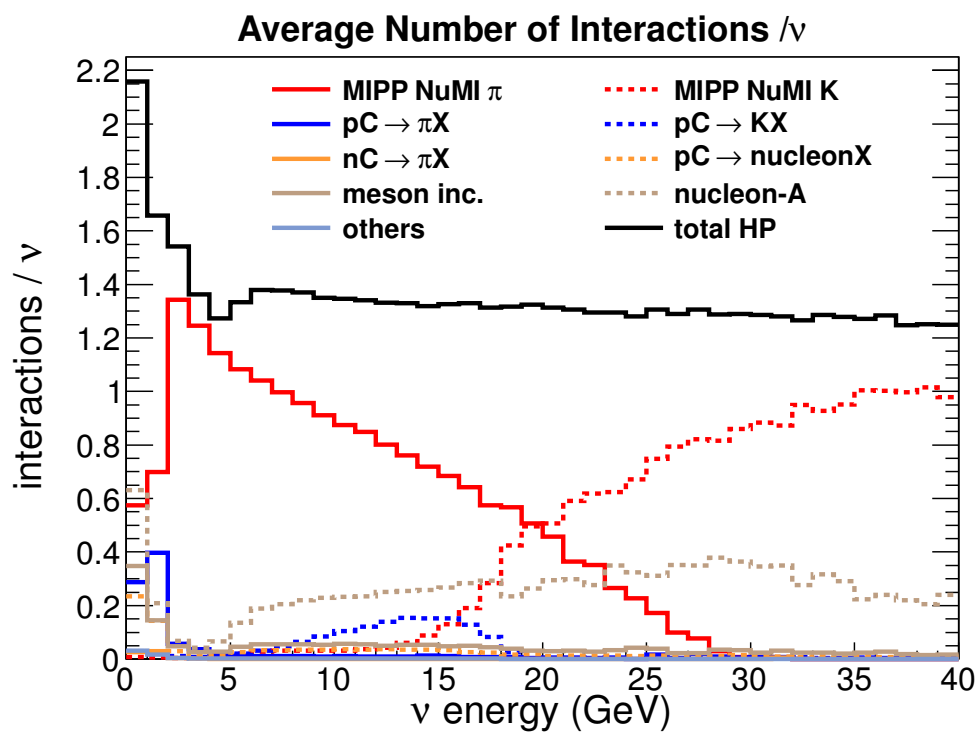

(a) $\nu_{\mu}$.

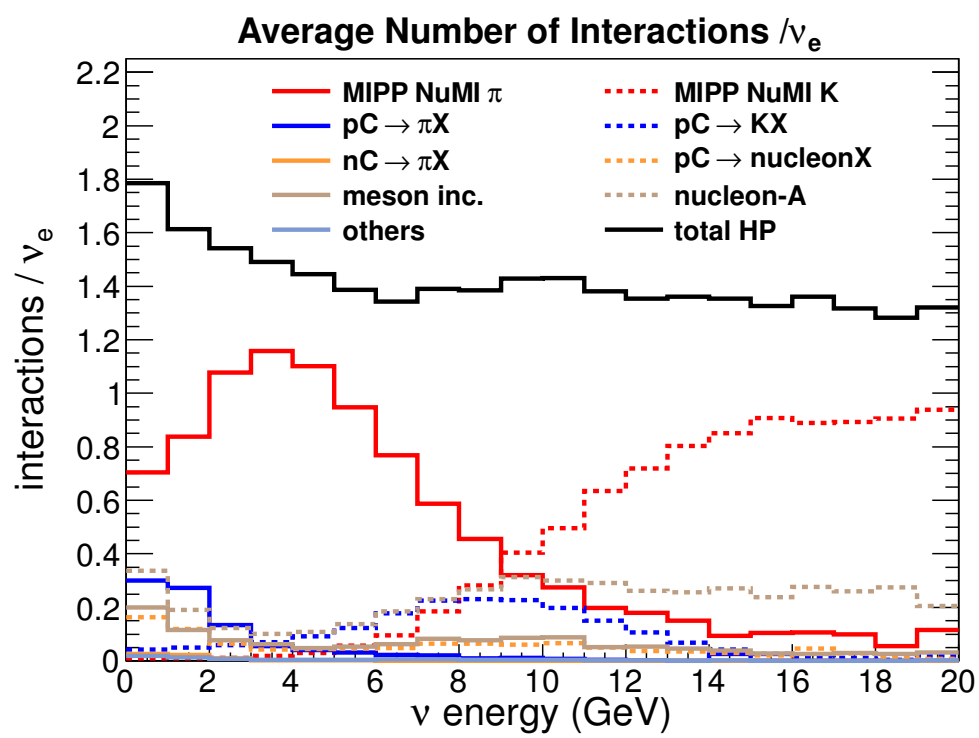

(b) $\nu_{e}$.

FIG. 5.5: Gen2-thick average number of hadronic interactions per neutrino minerva13. 
material scaling procedure described in Section 4.2.3. This value is small in the focusing peak because more of the neutrino parents are produced in the target. For higher energies it is almost flat (at 0.11) due to primary and high $x_{F}$ secondary protons interacting outside the target. This line remains unchanged between Gen2-thin and Gen2-thick as expected.

- The quasi-elastic proton production outside of the target (dashed red line) remain unchanged as expected, and it is a very small component of nucleon-A.

- The incident nucleon with momentum less than $12 \mathrm{GeV}$ (dashed blue line) has a small contribution to nucleon-A and remain almost unchanged since it is either not covered by thin target data or it produces low energy pions outside the MIPP acceptance region.

- Some of the protons created in quasi-elastic interactions in the target are also going to interact in the target and produce pions or kaons covered by MIPP. That is why this component (solid red line) reduces its value by more than half in Gen2-thick when compared to Gen2-thin.

- The orange line represents the remaining nucleon production after applying the previous classifications.

- The category others represents any nucleon-A interaction not classified yet. As mentioned, it is significant in the region between the edge of the pion production and the rise of kaon production PPFX coverage (neutrino energies in 20-30 GeV). They are basically pions or kaons not covered by data (directly or by using the material scaling) and produced in any material (for Gen2-thin) or just outside of the target (for Gen2-thick).

Figure 5.7a shows the average material traversed by any hadron in the relevant NuMI volumes for the beam attenuation effect: TGT, IC, DVOL and DPIP per 


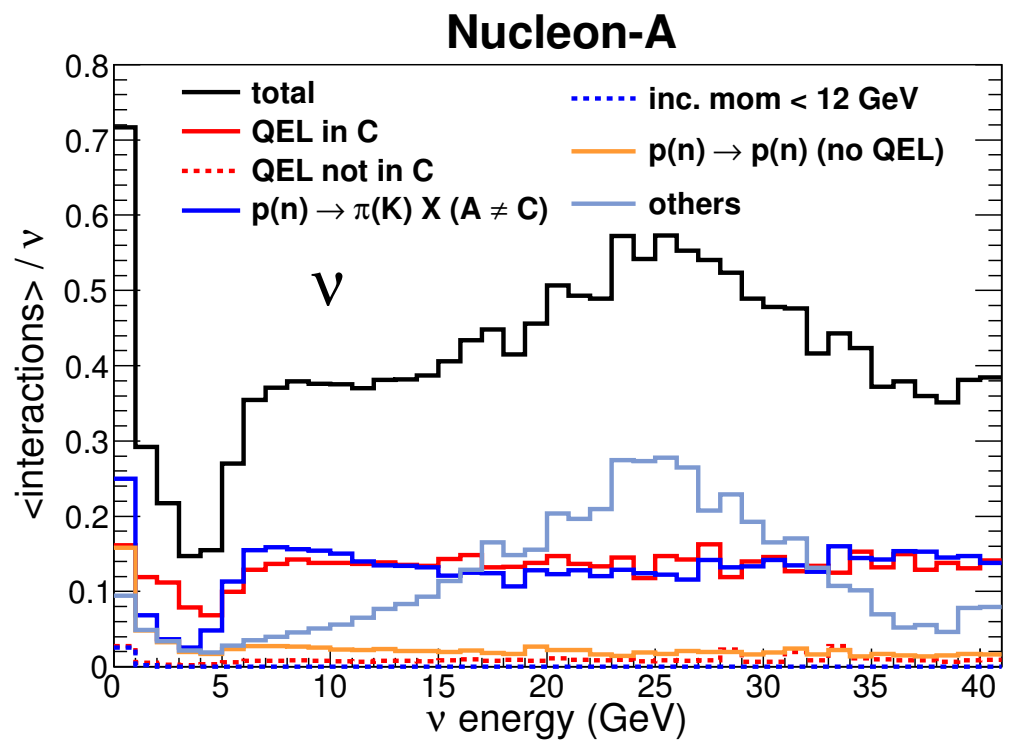

(a) Gen2-thin.

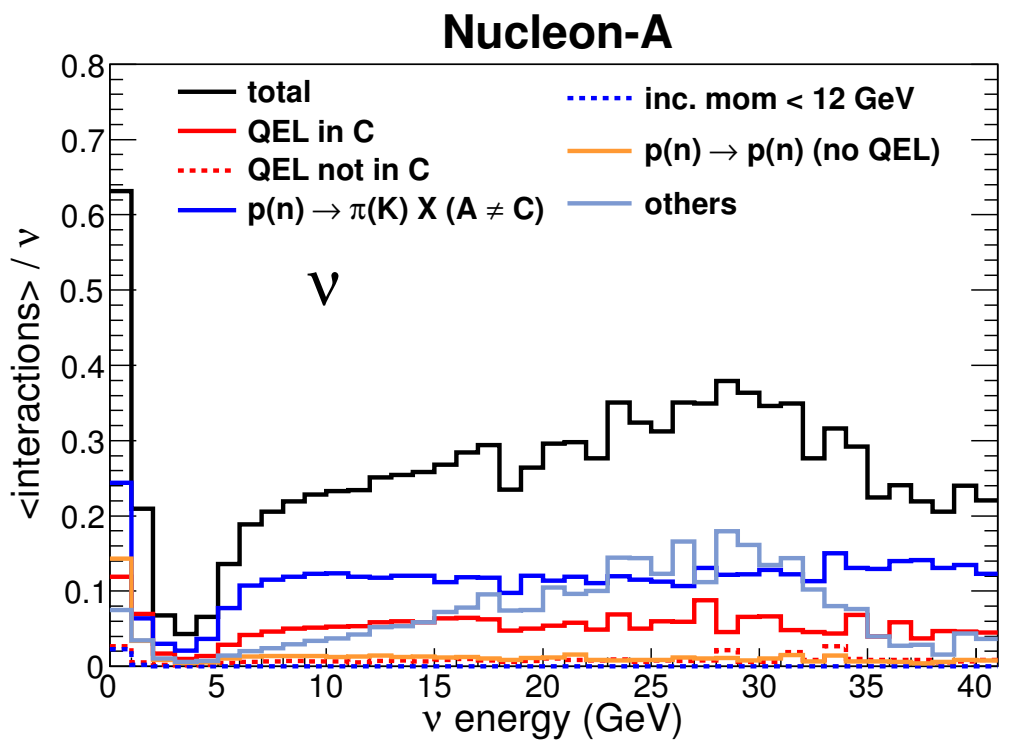

(b) Gen2-thick.

FIG. 5.6: Nucleon-A interaction per neutrino covered in Generation 2. 
$\nu_{\mu}$ with energies between $0-40 \mathrm{GeV}$ that pass through MINERvA detector. Gen2thin corrects the beam particle attenuation for the total average material traversed (black line) shown in the plot while Gen2-thick uses the primary proton passes through carbon (solid green line) and in materials different than carbon. The same information but just for pion neutrino parents is in Figure 5.7 restricted to the neutrino energy 0-20 GeV. Some characteristics to highlight are:

- For almost the whole neutrino energy range, the primary proton beam traversed about $40 \mathrm{~cm}\left(\sim 6 \mathrm{~mol} / \mathrm{cm}^{2}\right)$ in the target as average, except for neutrino energies around the focusing peak where the pions produced in the primary proton interactions are focused optimally: the average distance traveled by the primary protons decreases from $\sim 27 \mathrm{~cm}$ to $\sim 17 \mathrm{~cm}$ while for pions increases from $\sim 7 \mathrm{~cm}$ to $\sim 17 \mathrm{~cm}$ when the neutrino energy goes from $2 \mathrm{GeV}$ to $5 \mathrm{GeV}$.

- The pions traverse about $10 \mathrm{~cm}\left(\sim 1 \mathrm{~mol} / \mathrm{cm}^{2}\right)$ of $\mathrm{Al}$ on average. This is because the magnetic horns are long and thin and the pion angles are small and tend to travel more distance inside the horn inner conductors.

- Pion neutrino parents with energies greater than $\sim 4.5 \mathrm{GeV}$ (using the Equation 2.1 ) tend to reach the decay pipe and travel $\sim 200 \mathrm{~m}$ before decaying.

- As the neutrino energy increases, the material traversed by kaon becomes relevant.

\subsubsection{Results}

This subsection shows the flux results for:

- Gen2-thin, $\nu_{\mu}$, Figure 5.8,

- Gen2-thin, $\bar{\nu}_{\mu}$, Figure 5.10, 


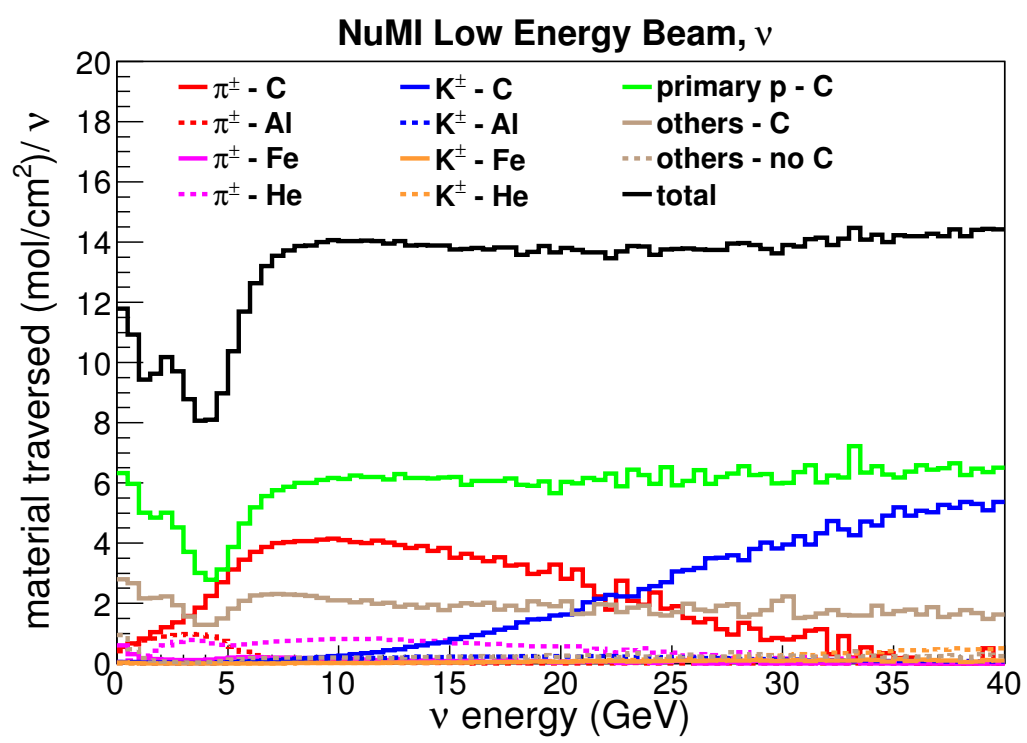

(a) All particles.

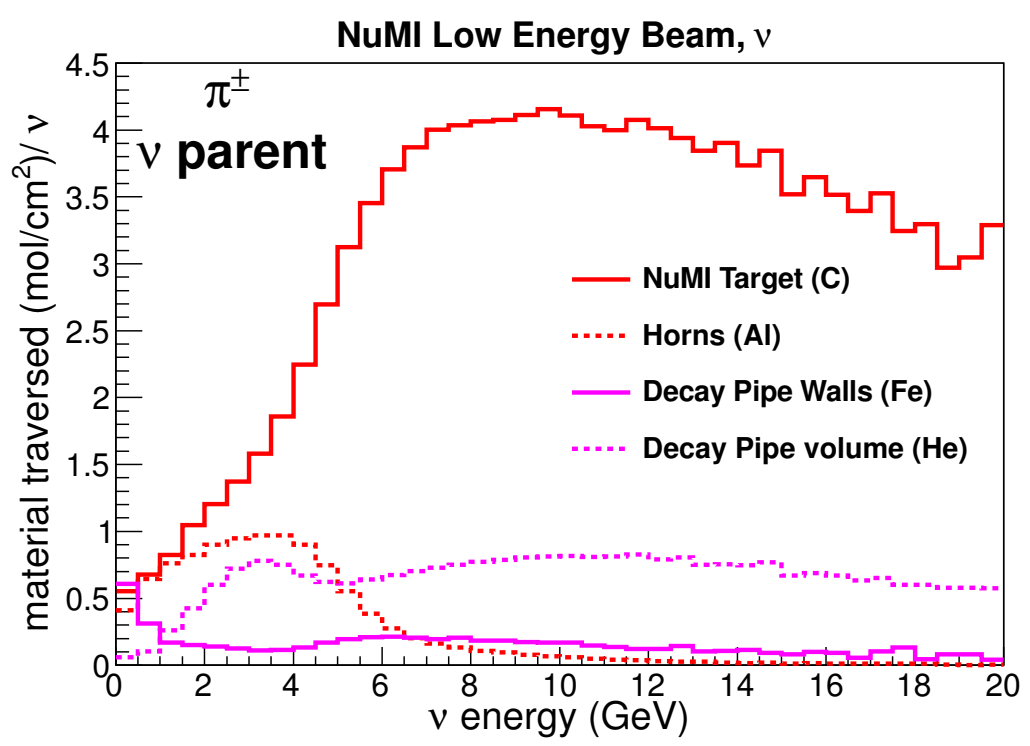

(b) Pions.

FIG. 5.7: NuMI material traversed. 
- Gen2-thin, $\nu_{e}$, Figure 5.12,

- Gen2-thick, $\nu_{\mu}$, Figure 5.9,

- Gen2-thick, $\bar{\nu}_{\mu}$, Figure 5.11,

- Gen2-thick, $\nu_{e}$, Figure 5.13.

The energy range is restricted to $0-20 \mathrm{GeV}$. The error bands combine hadron production and focusing uncertainties. The upper plots show the flux spectrum and the lower plot the ratio to the g4numi output in order to indicate the final correction we applied after using the tools developed in PPFX. A complete table, bin by bin, can be found in appendix B.

For $\nu_{\mu}$, the $\mathrm{MC}$ tends to underestimate the number of neutrino below $8 \mathrm{GeV}$ for Gen2-thin and below $11 \mathrm{GeV}$ for Gen2-thick (except at very low energies) and overestimate everywhere else. A discussion of the agreement between the two predictions can be found in Section 5.4, but it is clear that Gen2-thick predicts a big "dip" around 4-10 GeV of more than $20 \%$ while this effect in Gen2-thin is not that high.

The hadron production uncertainties for $\nu_{\mu}$ and $\nu_{e}$ in Gen2-thin are shown in Figure $5.14\left(\bar{\nu}_{\mu}\right.$ plots are not shown since they are similar to $\left.\nu_{\mu}\right)$ for an energy range of $0-20 \mathrm{GeV}$.

For Gen2-thin, the total uncertainties around the focusing peak are approximately $6-7 \%$ for both $\nu_{\mu}$ and $\nu_{e}$ due to the NA49 pion production data coverage. The uncertainties for $\nu_{\mu}$ increase to $\sim 9 \%$ for energies beyond $6 \mathrm{GeV}$ due to the influence of the nucleon-A correction, however, $\nu_{e}$ uncertainties increase in the same region because the kaon production becomes relevant. In general, the uncertainties are below $4 \%$ for the interactions where we applied data directly. The attenuation in TGT and other materials (it is called "absorption" in plots) has an uncertainty 


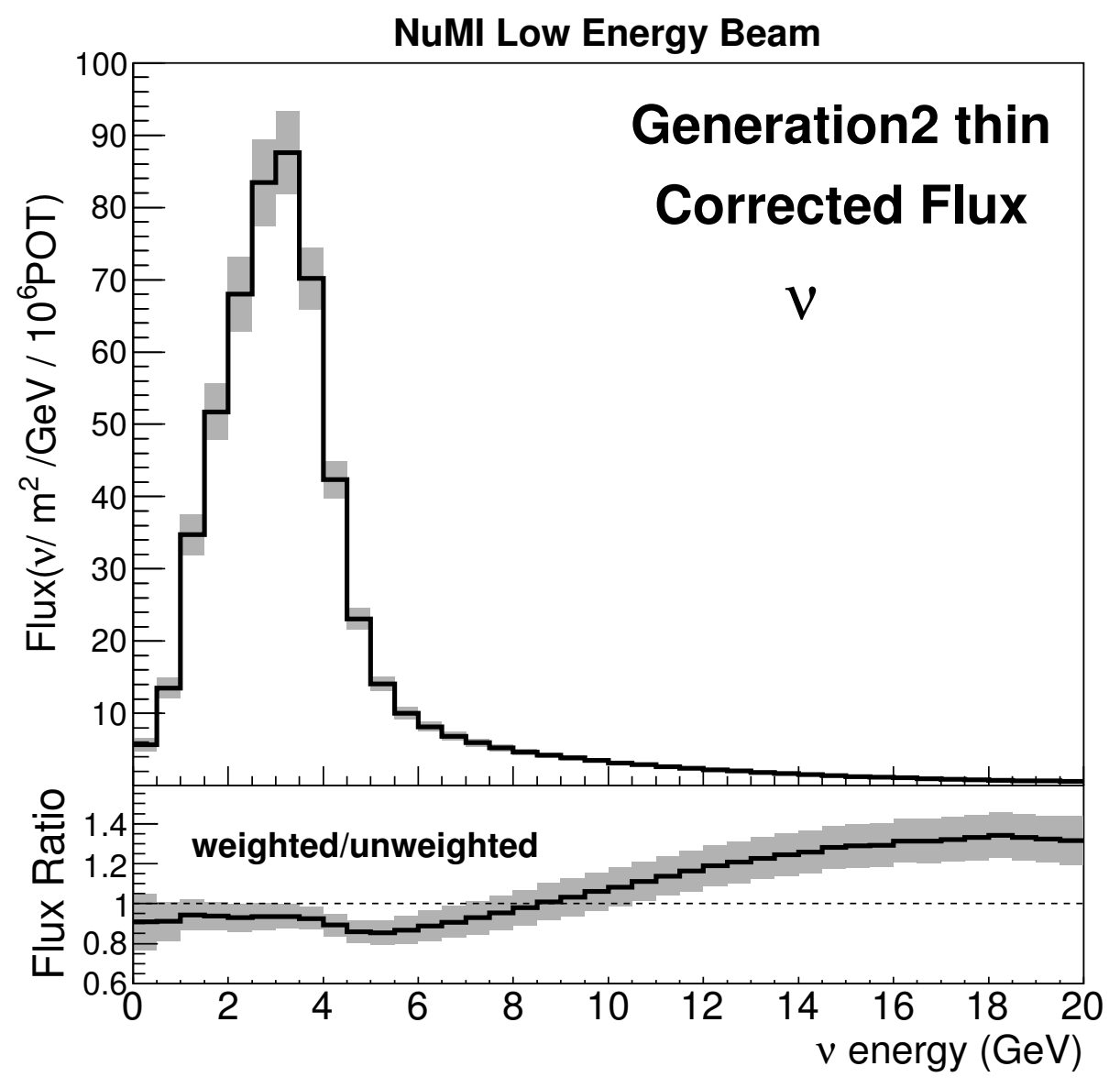

FIG. 5.8: Gen2-thin $\nu_{\mu}$ flux. 


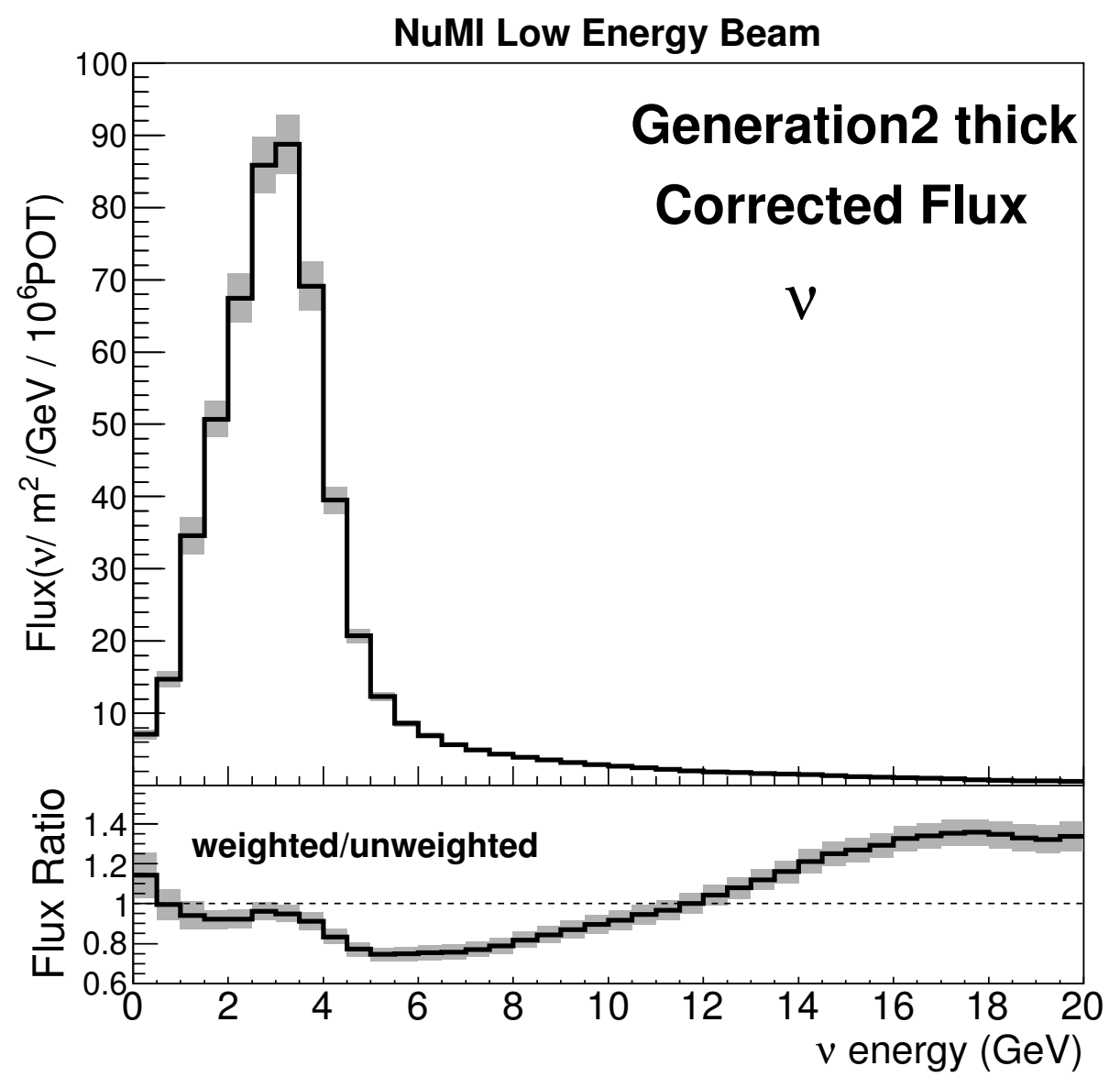

FIG. 5.9: Gen2-thick $\nu_{\mu}$ flux. 


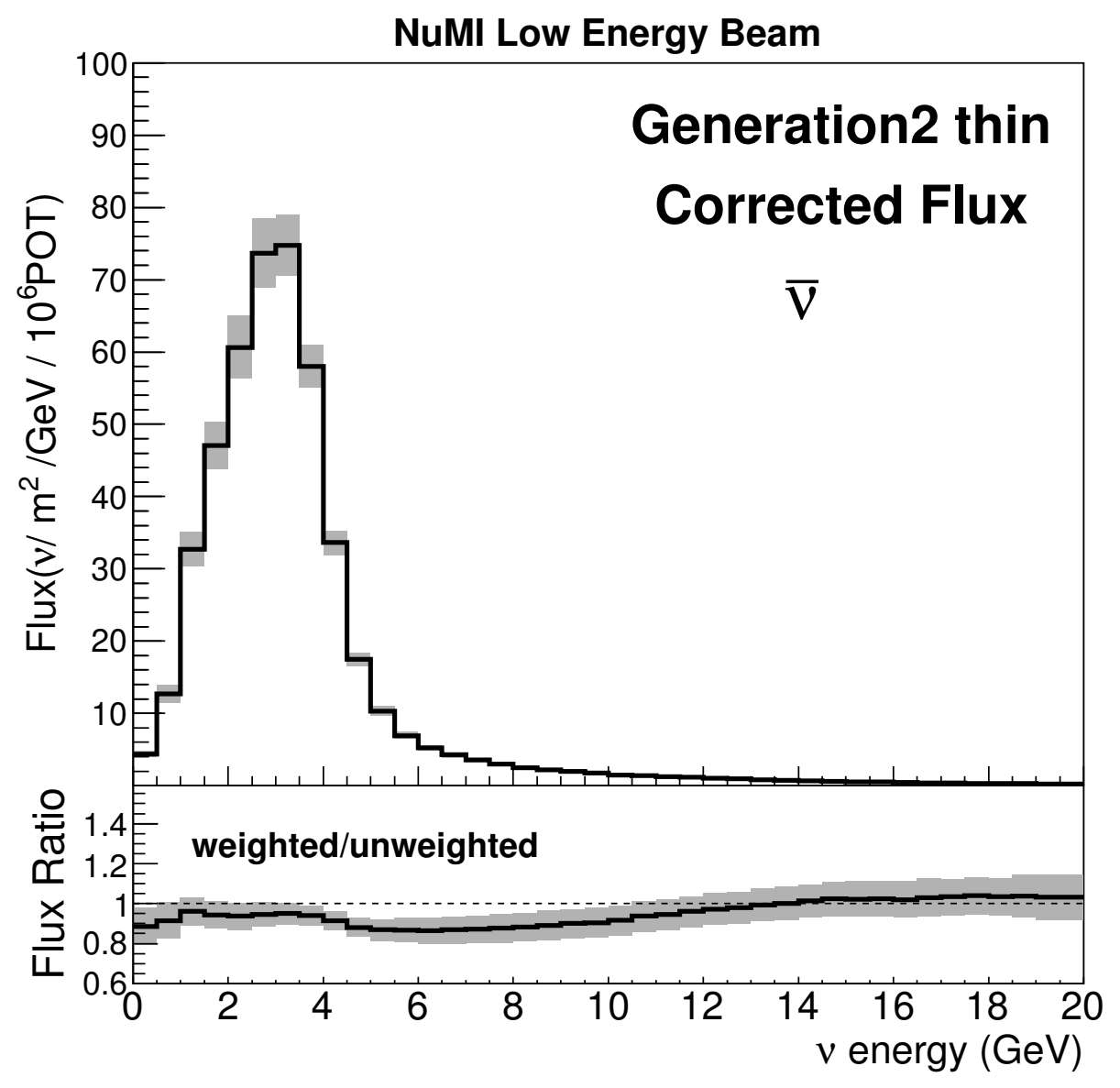

FIG. 5.10: Gen2-thin $\bar{\nu}_{\mu}$ flux. 


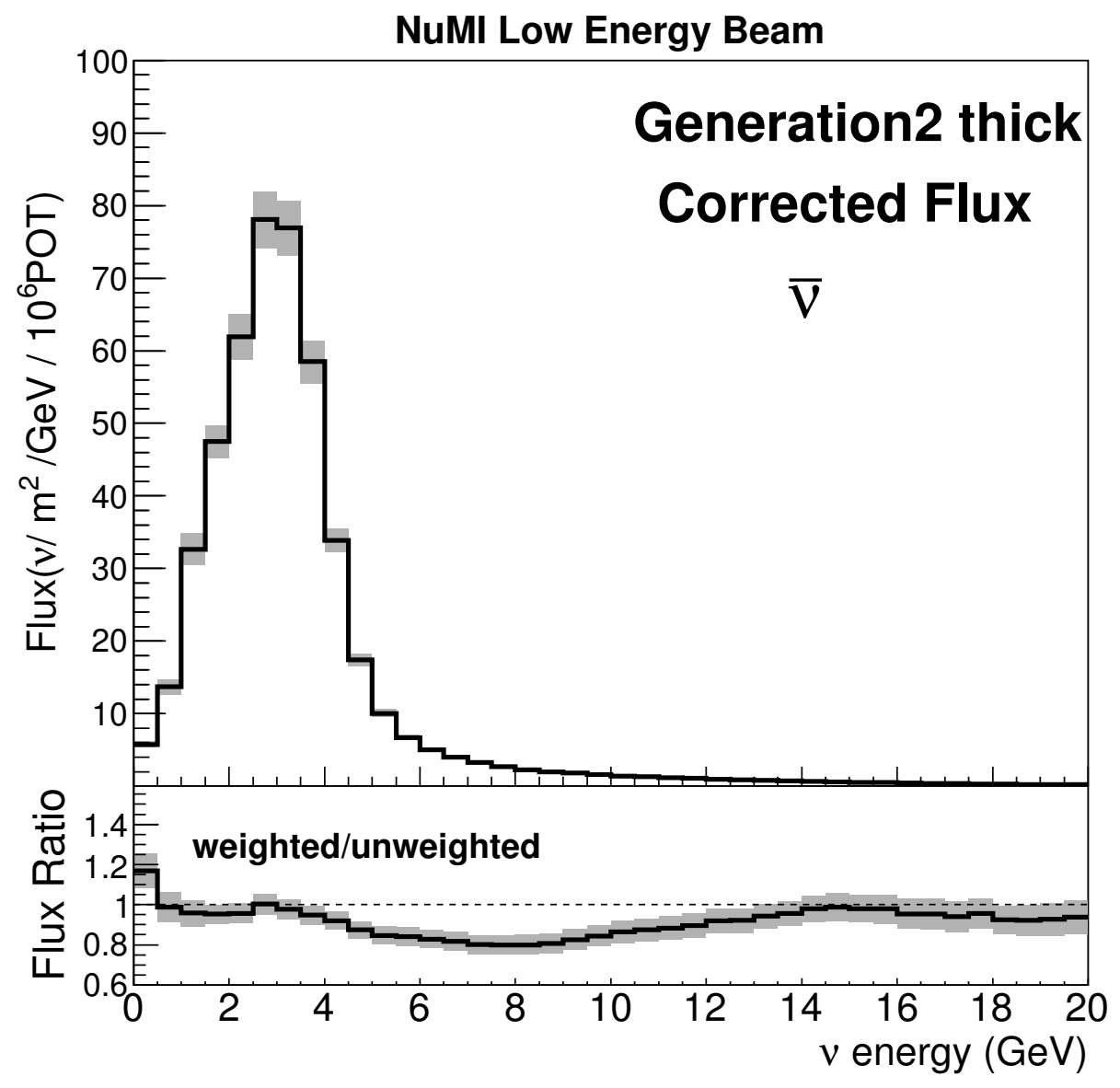

FIG. 5.11: Gen2-thick $\bar{\nu}_{\mu}$ flux. 


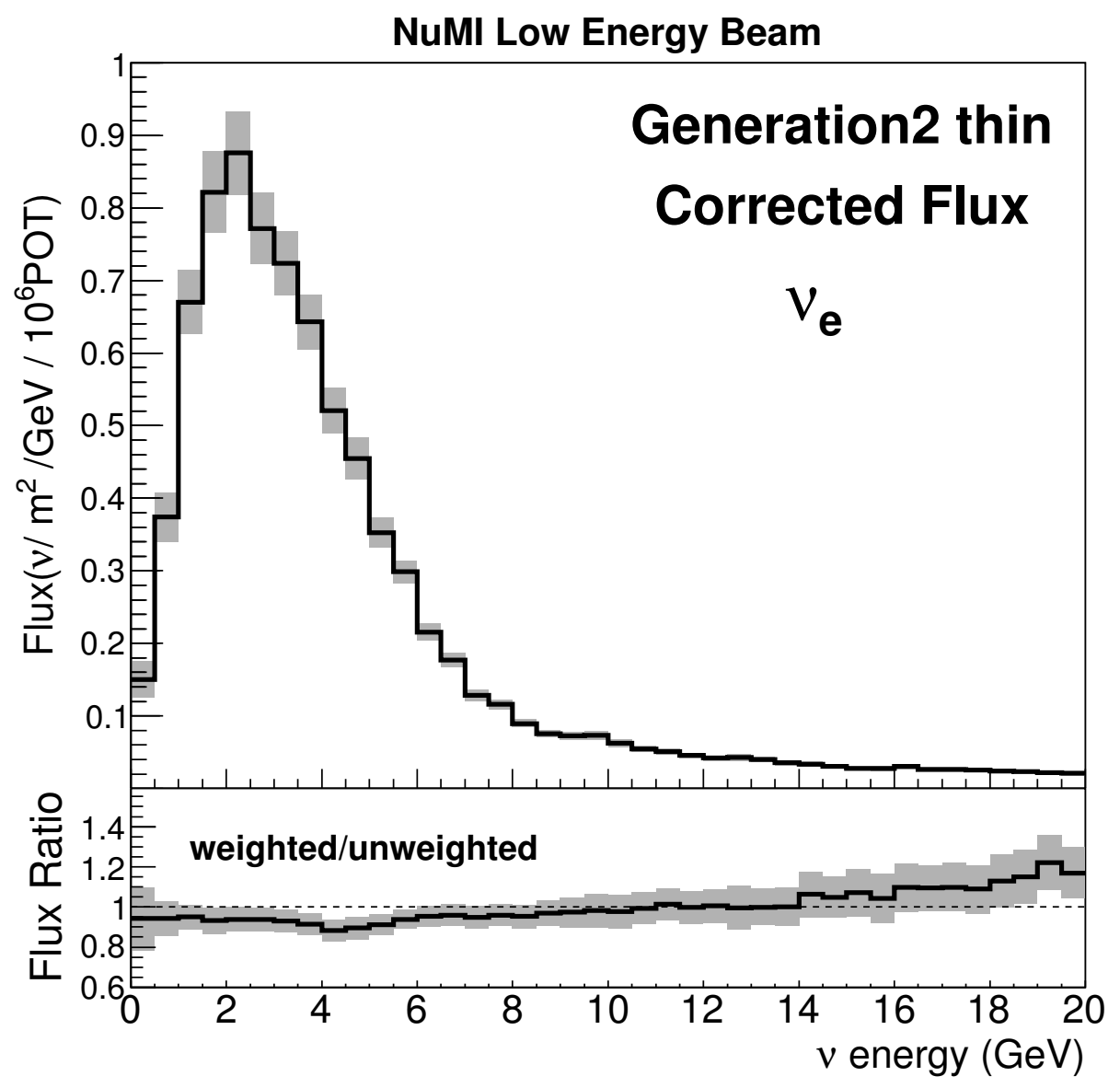

FIG. 5.12: Gen2-thin $\nu_{e}$ flux. 


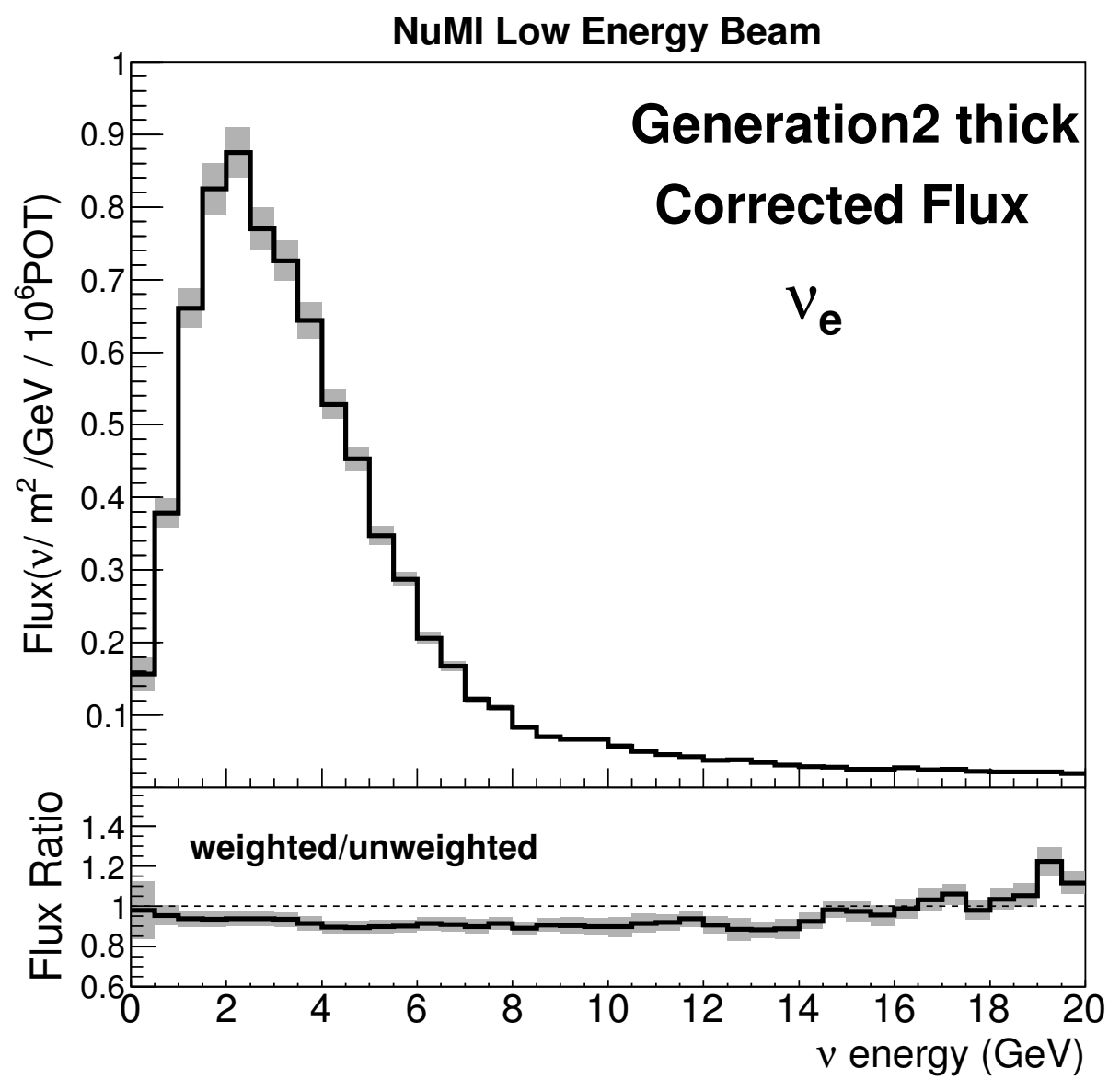

FIG. 5.13: Gen2-thick $\nu_{e}$ flux. 
of roughtly $3 \%$.

For Gen2-thick (see Figure 5.15), the total uncertainties are even smaller with an almost flat value of about $\sim 5 \%$. This is due to the MIPP NuMI data coverage. All the rest of the components reduce their value, except "absorption", since that is applied to volumes outside of the target.

The bin to bin energy correlation between $\nu_{\mu}, \bar{\nu}_{\mu}$ and $\nu_{e}$ is shown in Figure 5.16 for Gen2-thin and Gen2-thick. Some observations can be made from these plots:

- Bins with high correlations within each neutrino flavor reflect the highly correlated systematic uncertainty assumptions we have made for thin and thick target data and the contribution of different data bins to each neutrino energy bin.

- The correlation in the transition from lower to higher energies around 5-6 GeV is due to the focusing uncertainties.

- The correlation between $\nu_{\mu}$ and $\bar{\nu}_{\mu}$ comes from neutrinos with more than one hadronic interaction in their cascade history that share the same interaction for one or more ancestries. Comparing with Gen2-thick, when the $\nu_{\mu}$ and $\bar{\nu}_{\mu}$ correlation is very weak, we can conclude that these common interactions mainly happen inside of the target. 


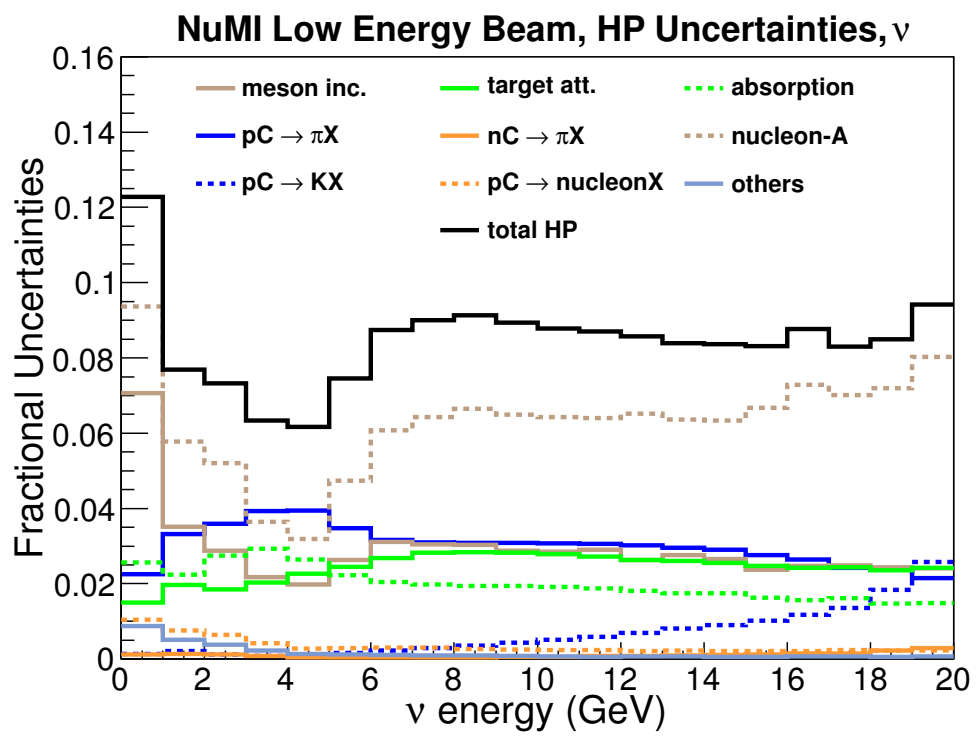

(a) $\nu_{\mu}$

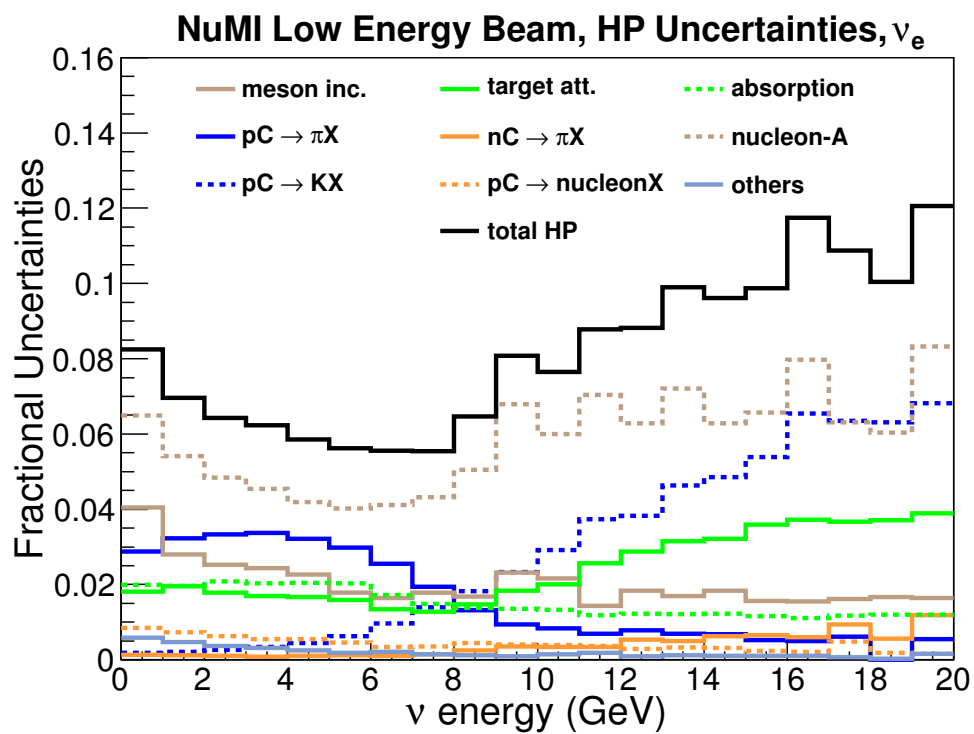

(b) $\nu_{e}$

FIG. 5.14: Fractional uncertainties for Gen2-thin. 


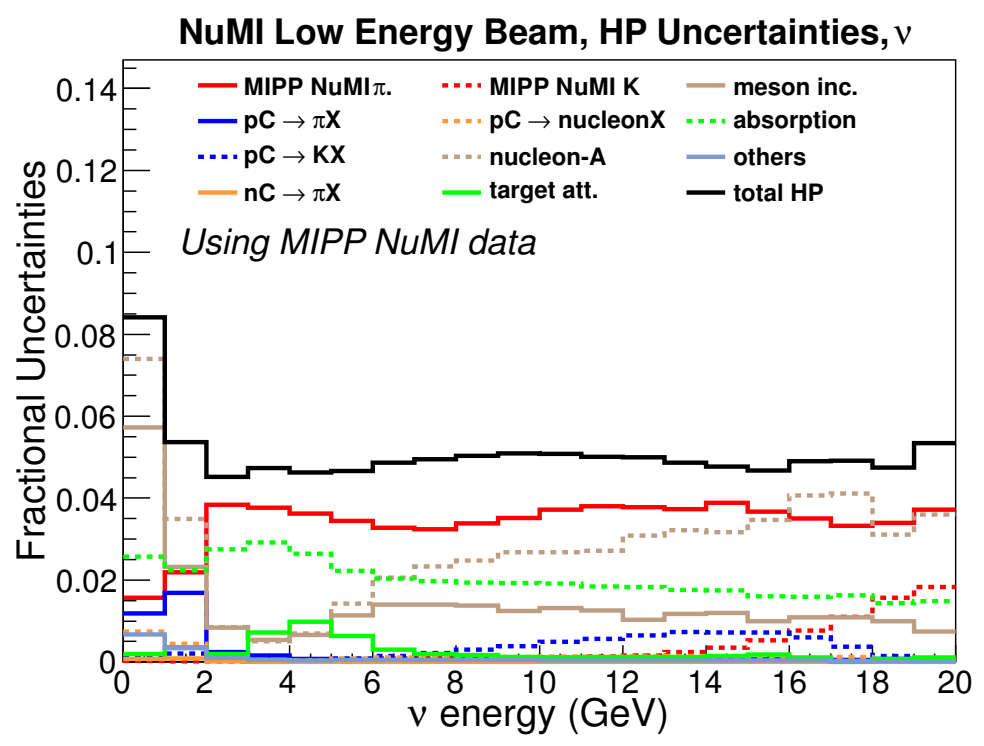

(a) $\nu_{\mu}$

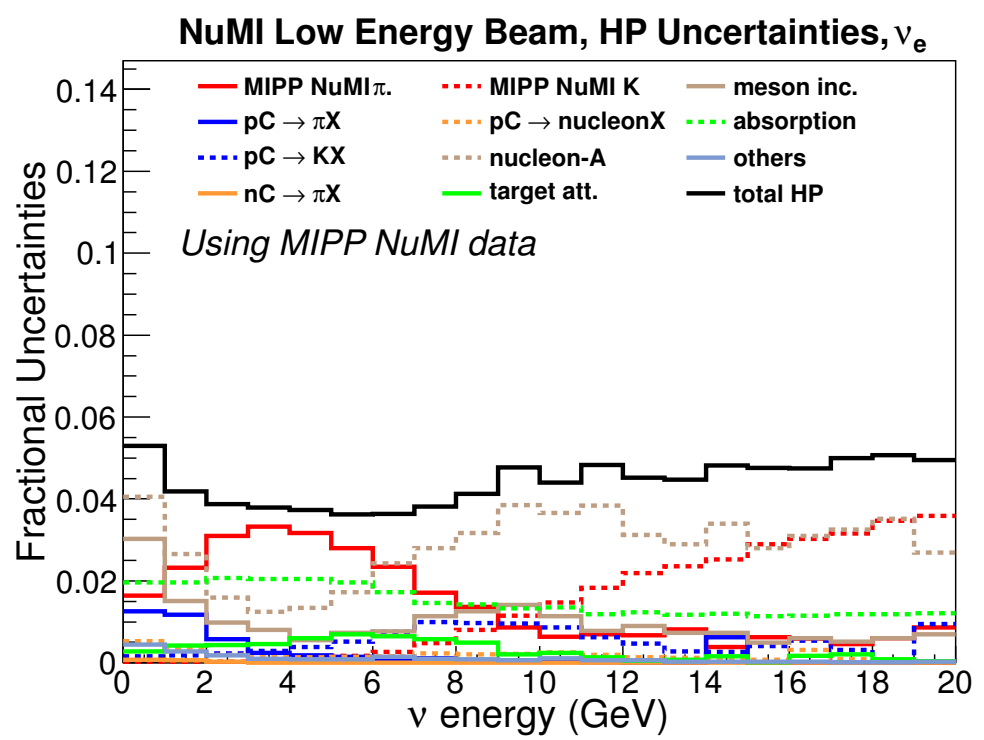

(b) $\nu_{e}$

FIG. 5.15: Fractional uncertainties for Gen2-thick. 


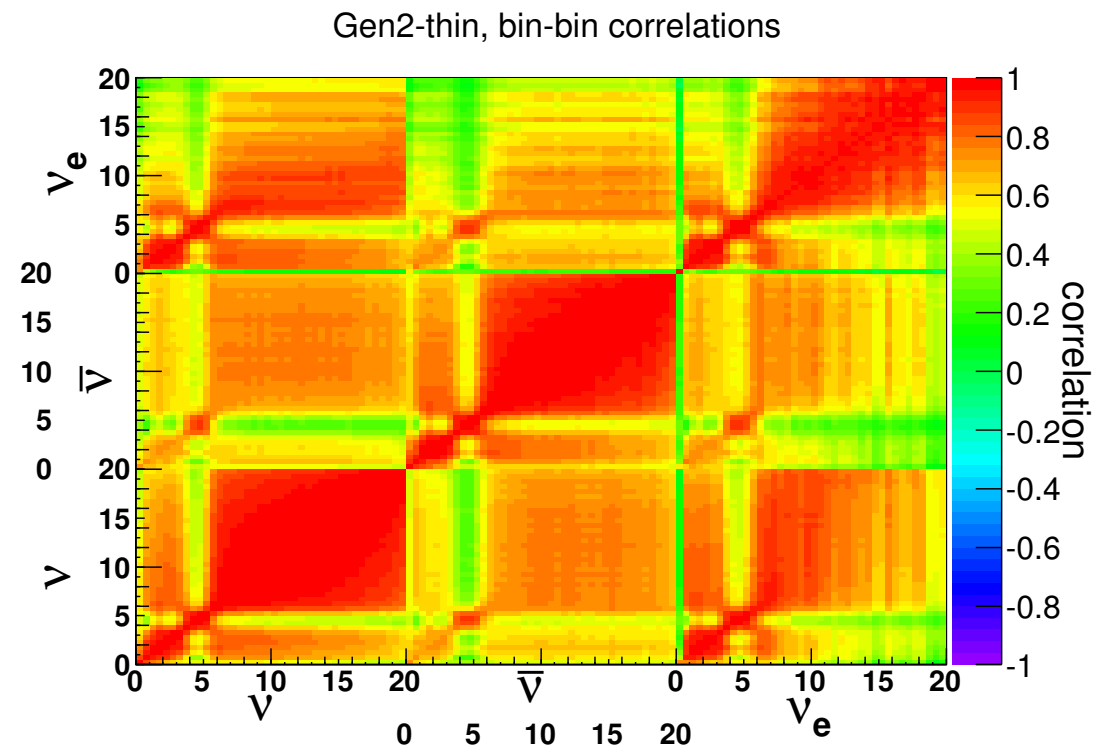

(a) Gen2 - thin

Gen2-thick, bin-bin correlations

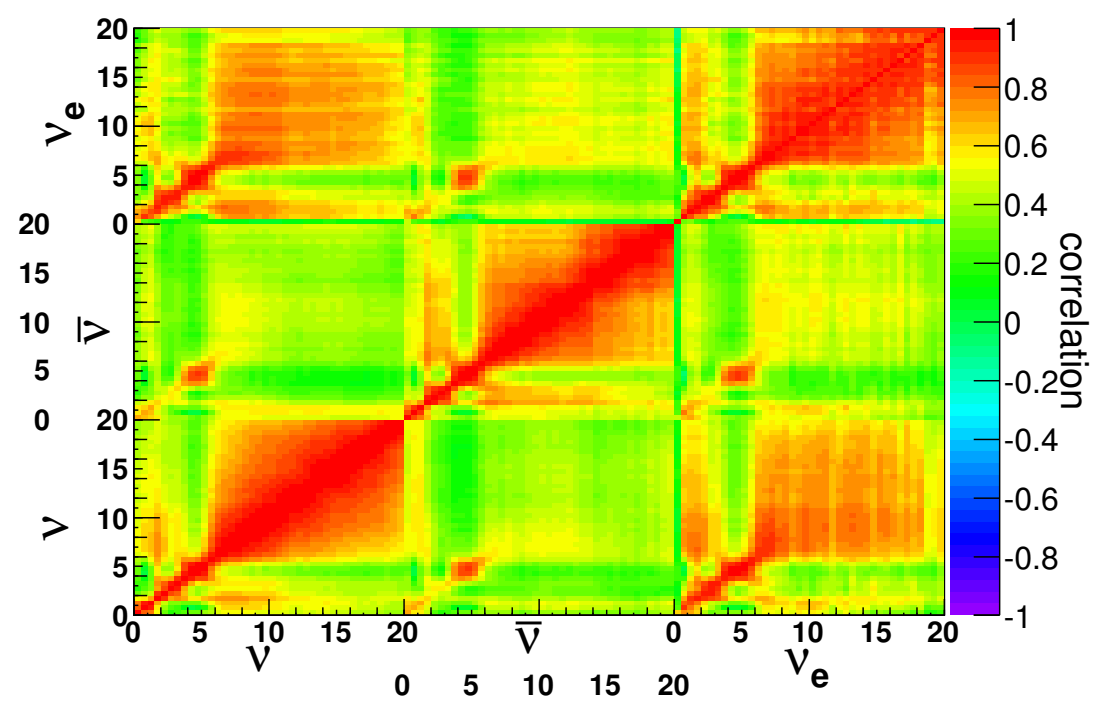

(b) Gen2 - thick

FIG. 5.16: Energy bin to bin flux correlation. 


\subsection{Discussion}

The flux integrated values over $0-20 \mathrm{GeV}$ are shown in Table 5.2 for $\nu_{\mu}, \bar{\nu}_{\mu}$ and $\nu_{e}$. Gen2-thin and Gen2-thick agree at the 1 sigma level. The uncertainties in the integrated flux have similar values around the focusing peak of the flux spectrum as we expect. The correlations between $\nu_{\mu}$ and $\bar{\nu}_{\mu}$ are shown in Figures 5.17 and 5.18.

\begin{tabular}{|l|c|c|}
\hline & integral $\left(\nu / m^{2} / 10^{6} \mathrm{POT}\right)$ & Uncertainty $(\%)$ \\
\hline Gen2-thin $\nu_{\mu}$ & 287.0 & 7.78 \\
\hline Gen2-thick $\nu_{\mu}$ & 280.8 & 5.37 \\
\hline Gen2-thin $\bar{\nu}_{\mu}$ & 233.5 & 7.46 \\
\hline Gen2-thick $\bar{\nu}_{\mu}$ & 238.6 & 5.51 \\
\hline Gen2-thin $\nu_{e}$ & 4.11 & 7.06 \\
\hline Gen2-thick $\nu_{e}$ & 4.07 & 4.93 \\
\hline \hline
\end{tabular}

TABLE 5.2: Integrated flux for $\nu_{\mu}$ and $\bar{\nu}_{\mu}$ in Gen2-thin and Gen2-thick for neutrino energy in $0-20 \mathrm{GeV}$.

The ratio of Gen2-thick over Gen2-thin flux spectra in Figures 5.19 and 5.20 show the level of consistency between both flux predictions. The ratios have been made by canceling all possible correlations in order to be sensitive to the real size of any disagreement between the two versions. We expect that if they agree perfectly, the ratios should be compatible with 1 , within errors.

For $\bar{\nu}_{\mu}$, both predictions agree within errors. However, for $\nu_{\mu}$, there is an energydependent disagreement that is greater than 1 sigma in two energy regions: $5-13 \mathrm{GeV}$ and $20-35 \mathrm{GeV}$. The former region is crucial to understand since it covers the falling edge of the focusing peak and most of the MINERvA analysis are restricted to energies below $20 \mathrm{GeV}$. Table 5.20 shows the bins where the disagreement is more than $1 \sigma$ for $\nu_{\mu}$ below $30 \mathrm{GeV}$. This conflict will be explored and resolved in the 


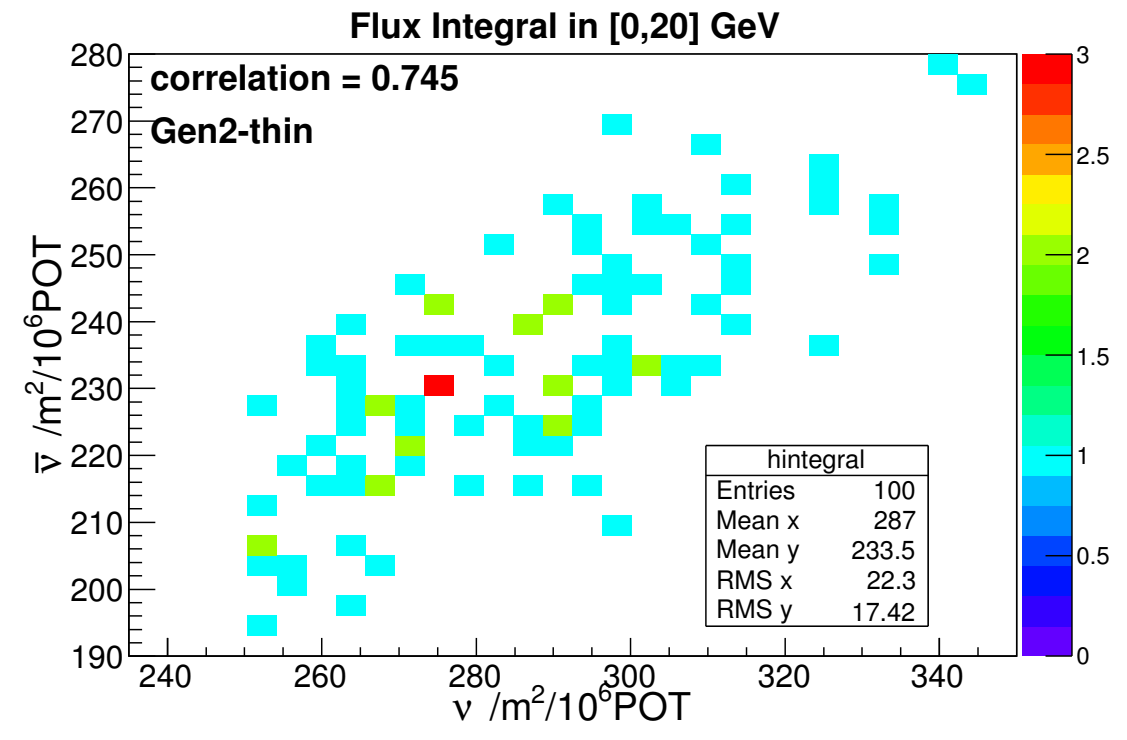

FIG. 5.17: Gen2-thin flux integral correlation between $\nu_{\mu}$ and $\bar{\nu}_{\mu}$. Each entry in this histogram is one universe.

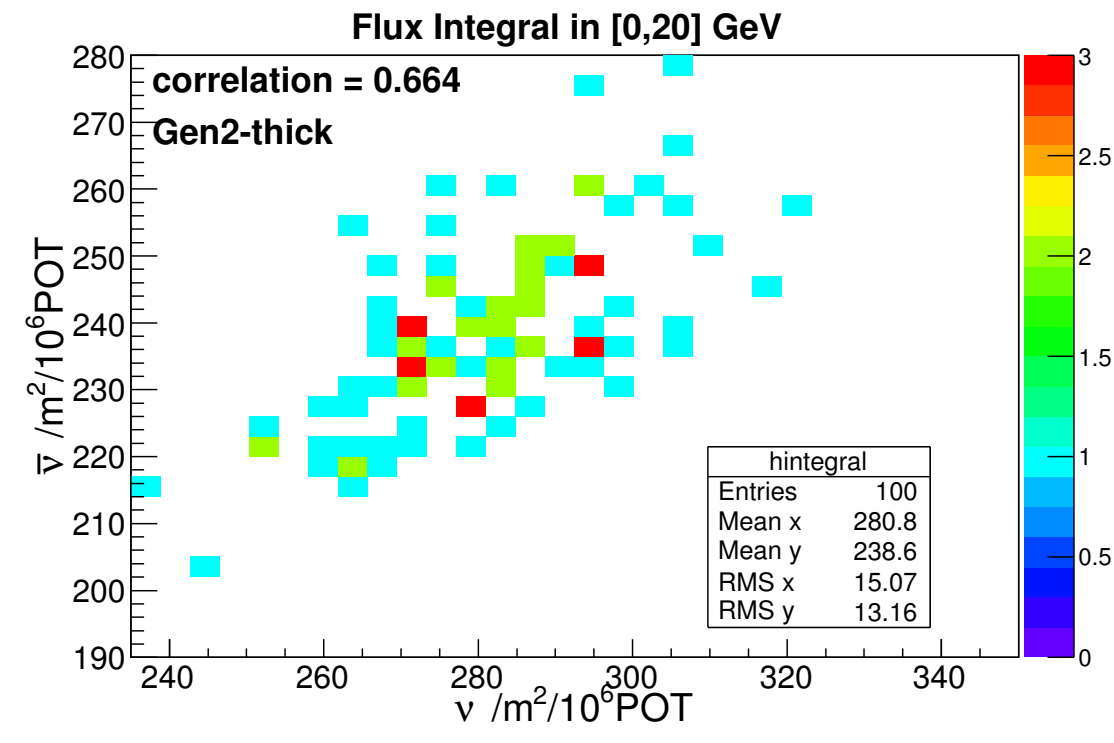

FIG. 5.18: Gen2-thick flux integral correlation between $\nu_{\mu}$ and $\bar{\nu}_{\mu}$. Each entry in this histogram is one universe. 


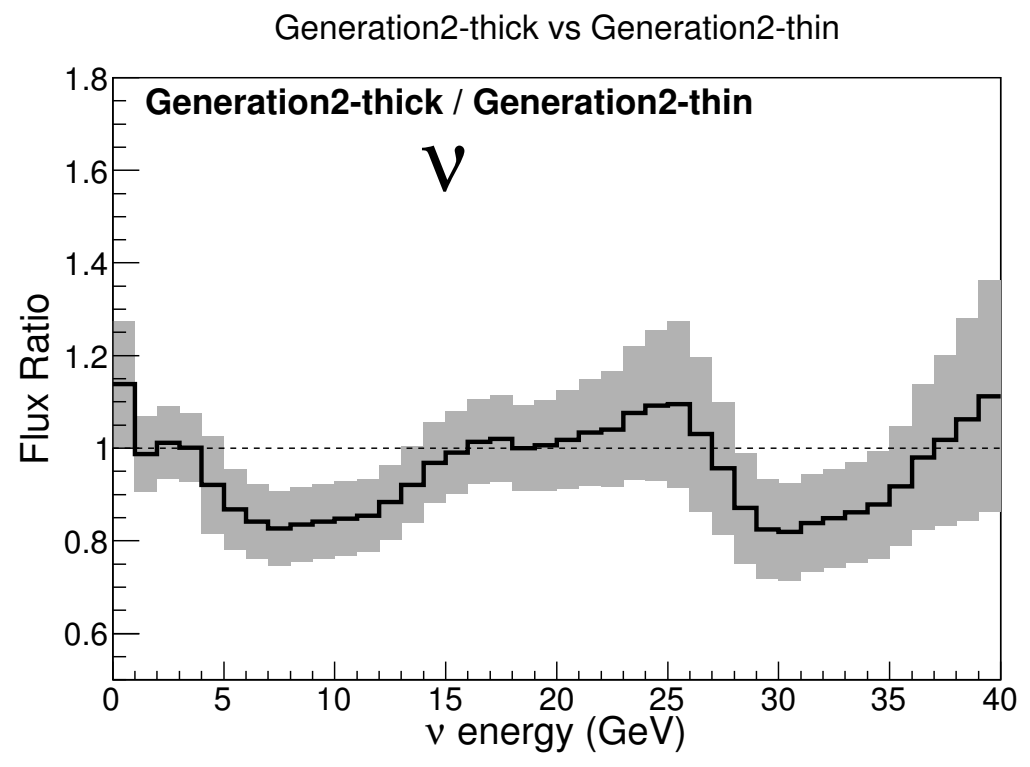

FIG. 5.19: $\nu_{\mu}$ Gen2-thin and Gen2-thick comparison.

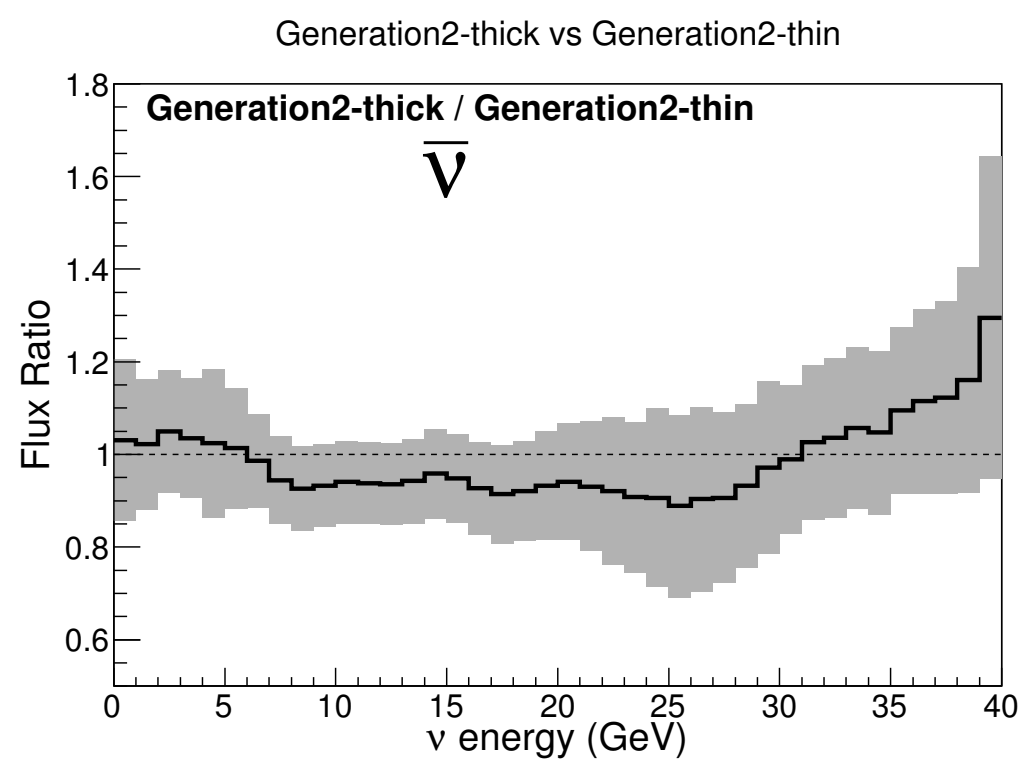

FIG. 5.20: $\bar{\nu}_{\mu}$ Gen2-thin and Gen2-thick comparison. 
next chapter.

\begin{tabular}{|l|c|c|c|c|c|c|c|c|}
\hline$\Delta E(\mathrm{GeV})$ & $5-6$ & $6-7$ & $7-8$ & $8-9$ & $9-10$ & $10-11$ & $11-12$ & $12-13$ \\
\hline$\sigma$ & 1.52 & 1.97 & 2.16 & 2.04 & 1.96 & 1.90 & 1.86 & 1.44 \\
\hline \hline
\end{tabular}

TABLE 5.3: Disagreement between Gen2-thin and Gen2-thick for $\nu_{\mu}$. 


\section{CHAPTER 6}

\section{Additional flux constraining using MINERvA in-situ measurements}

At the end of the last chapter, we showed a potential disagreement between the Gen2-thin and Gen2-thick the $\nu_{\mu}$ flux predictions. Is it possible to reconcile these two predictions, or favor one of them in a physics-motivated way? The first section of this chapter addresses this question.

The strongly energy-dependent ratio between Gen2-thick and Gen2-thin $\nu_{\mu}$ fluxes (Figure 5.19) suggests that the best approach is to use the flux predicted from low-nu, since it is essentially a flux shape measurement. Furthermore, as we saw in Table 5.2 shows, the flux integrated in all neutrino types agree each with other to within their uncertainties, meaning that the neutrino - electron scattering constraint does not suitably solve this potential disagreement. However, the second section of this chapter is dedicated to exploring the application of the MINERvA results of this channel to reduce the flux uncertainties. 


\subsection{Low-nu flux at MINERvA}

The low-nu procedure was applied in MINERvA to calculate the $\nu_{\mu}$ and $\bar{\nu}_{\mu}$ fluxes and these fluxes in turn were used to extract inclusive scattering cross-sections [48]. The general principle of the method was explained in Section 3.3 and this section presents only the results and a brief summary of the procedure in order to make the comparisons with Generation 2 a priori results introduced in the previous chapter. More details can be found in Ref. [48].

The low-nu flux analysis was done using the Generation 1 flux prediction, but it is insensitive to the flux model, as one would hope. It uses three $\nu$ cuts that were applied to the inclusive scattering events in overlapping neutrino energies: $\nu<300 \mathrm{MeV}(E>2 \mathrm{GeV}), \nu<800 \mathrm{MeV}(E>5 \mathrm{GeV})$ and $\nu<2 \mathrm{GeV}(E>9 \mathrm{GeV})$.

Figure 6.1a shows the fraction of the inclusive data sample (overlap) with $\nu$ less than the cut for $\nu_{\mu}$ in the LE010z185i configuration ${ }^{1}$.

After applying a Bayesian unfolding to account for the detector resolution and after applying efficiency corrections, the three event rate samples under the $\nu$ cuts have, basically, the shape of the flux. The next steps followed were:

1. $\nu$ cross-section $\left(\sigma_{\nu}\right)$. The neutrino nucleon charged current cross-section $\sigma_{\nu}$ is calculated independently in each of the three $\nu$ samples using Equation 1.12 and the a priori Generation 1 flux. Figure 6.1b shows these cross-sections for $\nu_{\mu}$.

2. Finite $\nu$. A correction for finite $\nu$ is made using GENIE by calculating:

$$
S\left(\nu_{0}, E\right)=\frac{\sigma\left(\nu<\nu_{0}, E\right)}{\sigma\left(\nu<\nu_{0}, E \rightarrow \infty\right)}
$$

where $\nu_{0}$ is a particular $\nu$ cut and $\mathrm{E}$ is the neutrino energy (see Equation 3.1).

\footnotetext{
${ }^{1}$ The plots corresponding to Figures 6.1 and 6.2 for $\bar{\nu}_{\mu}$ can be found in Ref. [48].
} 


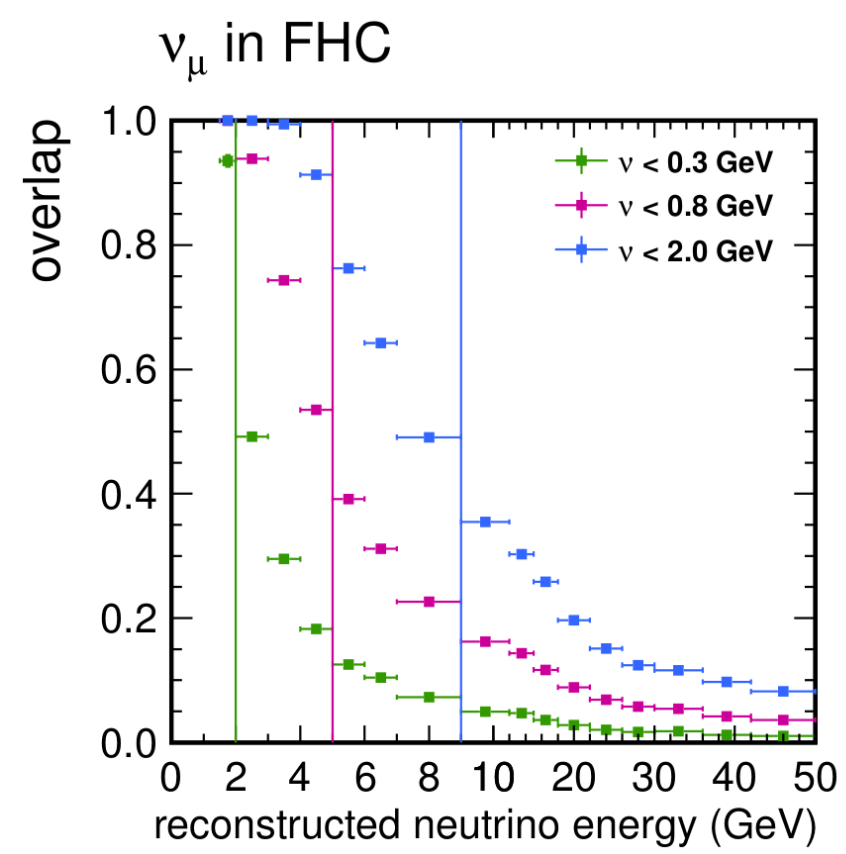

(a) Overlap.

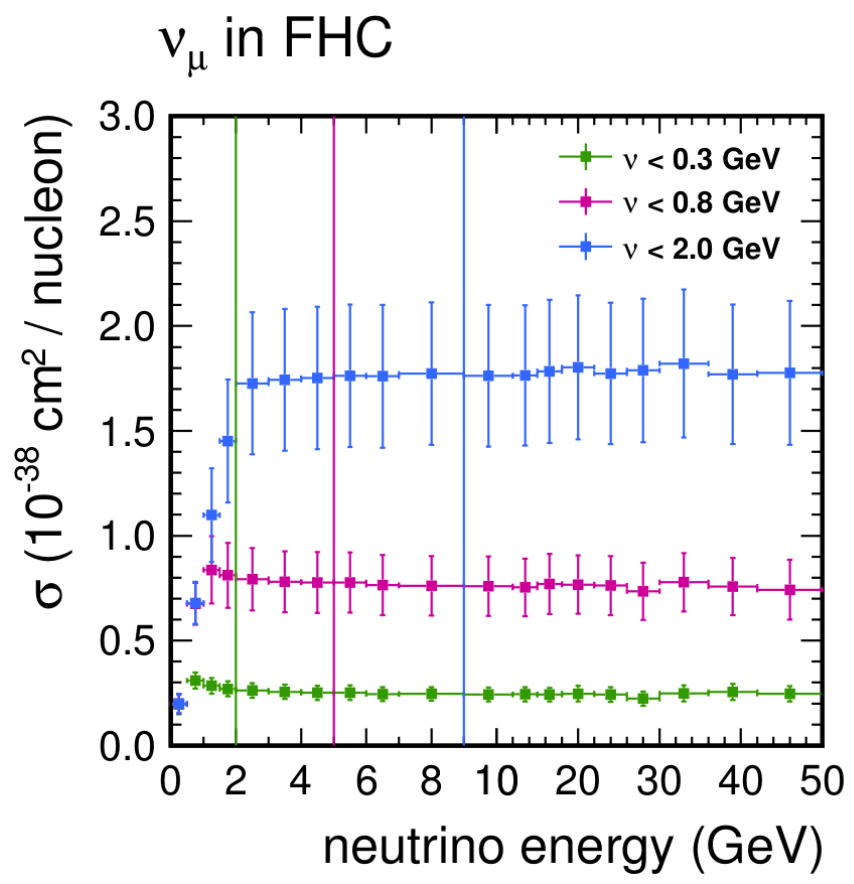

(b) $\nu$ cross-sections.

FIG. 6.1: Overlap and extracted $\nu$ cross-sections for $\nu_{\mu}$ in LE010z185i. Plots taken from [48]. 
3. Normalization. The extracted charged current cross-section is normalized to match the GENIE cross-section value in the $9-12 \mathrm{GeV}$ energy region ${ }^{2}$ and other normalization factors for all three $\nu$ samples are also calculated to agree with this match by following a fitting procedure between $\nu$ samples (fit errors are included as uncertainties).

4. Flux. The $\nu_{\mu}$ and $\bar{\nu}_{\mu}$ fluxes are extracted by applying the factors found in step 3 per $\nu$ sample and they are merged to get a flux determination in the 2-50 neutrino energy region in 15 bins. Table 6.1 contains a list of the bin conventions used in this analysis.

\begin{tabular}{|c|c||c|c||c|c|}
\hline Bin & range $(\mathrm{GeV})$ & Bin & range $(\mathrm{GeV})$ & Bin & range $(\mathrm{GeV})$ \\
\hline 1 & $2-3$ & 6 & $7-9$ & 11 & $22-26$ \\
2 & $3-4$ & 7 & $9-12$ & 12 & $26-30$ \\
3 & $4-5$ & 8 & $12-15$ & 13 & $30-36$ \\
4 & $5-6$ & 9 & $15-18$ & 14 & $36-42$ \\
5 & $6-7$ & 10 & $18-22$ & 15 & $42-50$ \\
\hline
\end{tabular}

TABLE 6.1: Low-nu binning convention.

The flux results following this procedure (for brevity we called it "low-nu flux") are shown in Figure 6.2a for $\nu_{\mu}$ in the LE010z185i configuration $\left(\bar{\nu}_{\mu}\right.$ flux can be found in Ref. [48]). The spectrum is shown at the right and the data/MC ratio on the left. The black markers in the plots correspond to the extracted low-nu flux and the red line is the Generation 1 flux prediction. The total fractional uncertainty, shown in Figure $6.2 \mathrm{~b}$, is between $8-11 \%$. The contribution from the a priori flux to the uncertainty in this calculation is small.

\footnotetext{
${ }^{2}$ This step requires us to use precise external measurements. This analysis used GENIE since this is itself based on data.
} 

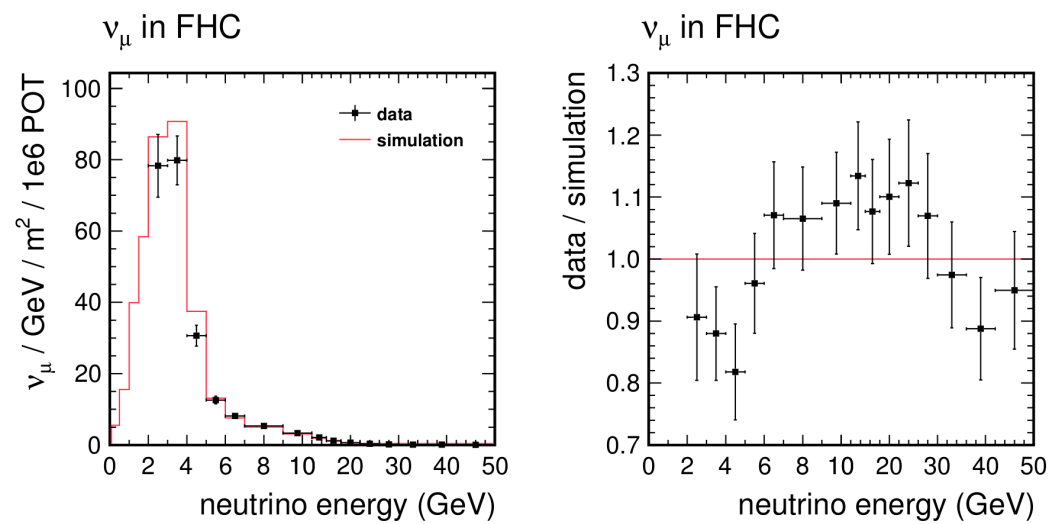

(a) Flux.

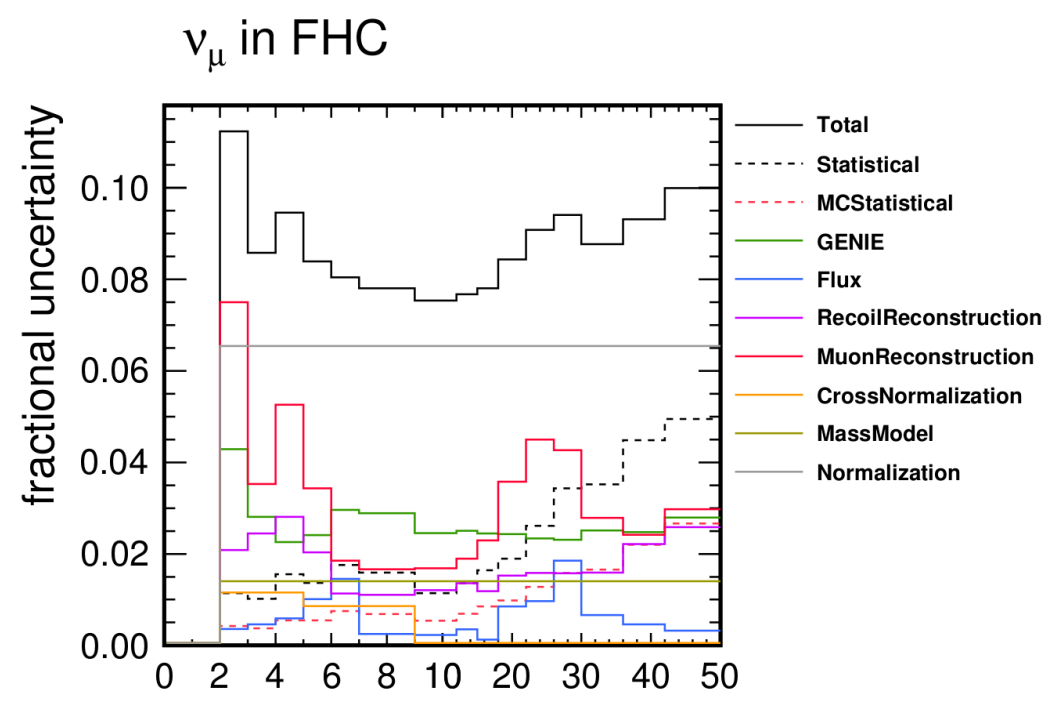

(b) Fractional uncertainties.

FIG. 6.2: $\nu_{\mu}$ flux and its fractional uncertainties in LE010z185i calculated using the low-nu technique in MINERvA. Plots taken from [48]. 


\subsubsection{Generation 2 vs low-nu flux comparison}

Figures 6.3 and 6.4 show a comparison between the Generation 2 fluxes (thick and thin) to the low-nu flux for $\nu_{\mu}$. In the ratio plots (lower side), the uncertainties have been computed by adding in quadrature the Generation 2 and low-nu uncertainties ${ }^{3}$. In principle, adding in quadrature is wrong as it does not account for correlations due to the flux model. However, the flux uncertainty component of the low-nu results is negligible, so the overestimation of these ratio uncertainties is small.

A first "by eye" conclusion is that both Gen2-thick and Gen2-thin agree with the low-nu result around the focusing peak and for high energies, but Gen2-thick disagrees with low-nu in the 5-15 GeV neutrino energy range while Gen2-thin does not. Since the neutrino energy bins are highly correlated (see Figure 5.16), a more careful approach is needed. MINERvA conducted a quantitative study using a $\chi^{2}$ test [92] fully including the correlations between both fluxes.

The idea is to test the hypothesis $\left(H_{0}\right)$ that $L-G=0$, where $G$ and $L$ are the Generation 2 (thin or thick) and the low-nu fluxes. A $\chi^{2}$ is constructed by:

$$
\chi^{2}=\sum_{i, j ; i \leq j}^{N}\left(G_{i}-L_{i}\right)\left(G_{j}-L_{j}\right) V_{i j}^{-1}
$$

where the sum is made bin by bin $(\mathrm{i}, \mathrm{j})$ for $G_{i, j}-L_{i, j}$ and the matrix $V=V_{G}+V_{L}$ is the sum of the Generation 2 and low-nu covariance matrices. The results for two neutrino energy ranges, 2-50 GeV and 2-22 GeV, are summarized in Table 6.2. Looking at both ranges, we conclude that Gen2-thin is in a good agreement with low-nu while Gen2-thick has poor agreement (especially when we consider the whole energy region).

Similar work has been conducted in [92] to test the agreement between Gen2-

\footnotetext{
${ }^{3}$ Currently, we do not have the technology to cancel common correlations.
} 


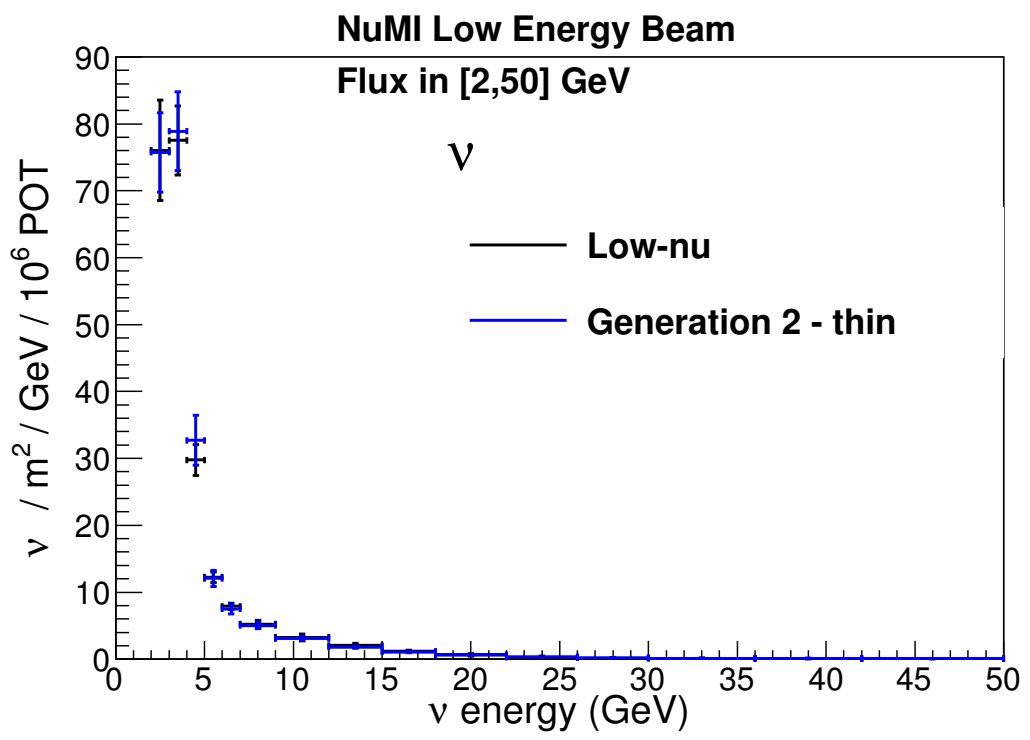

(a) Flux spectra.

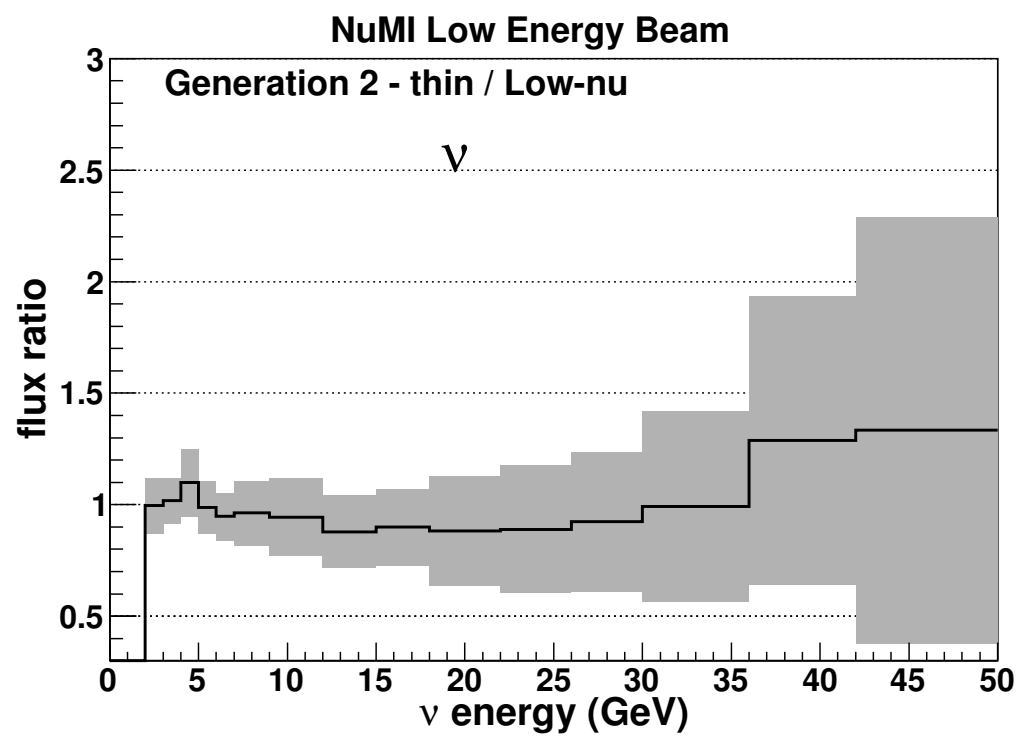

(b) Ratios.

FIG. 6.3: Gen2-thin $\nu_{\mu}$ flux vs low-nu. 


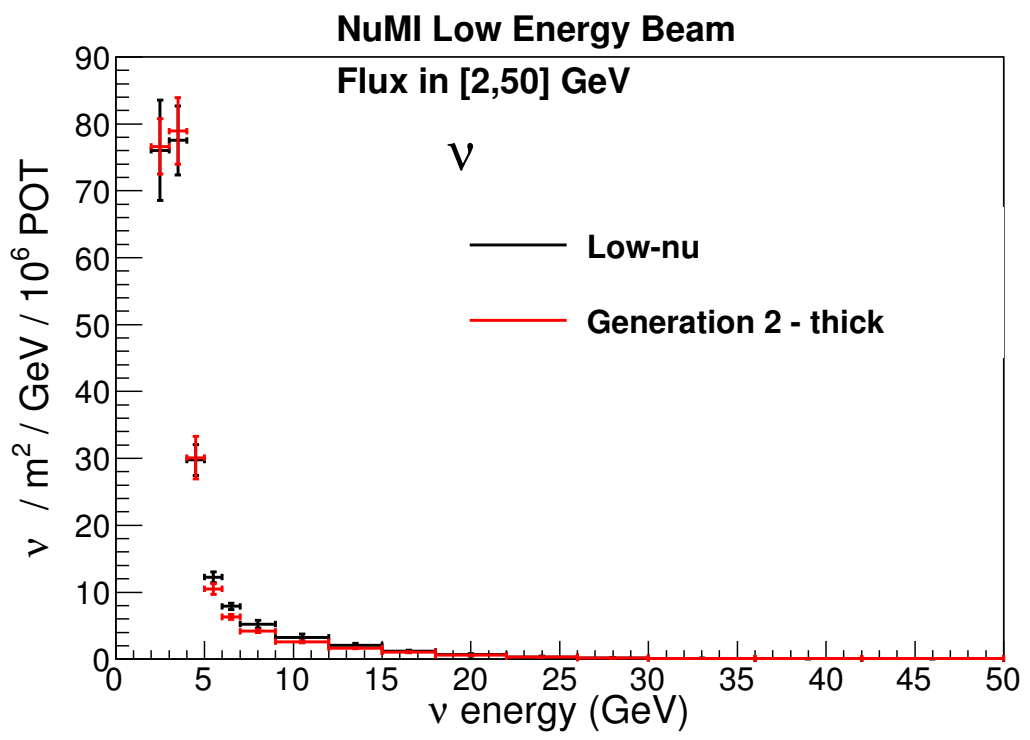

(a) Flux spectra.

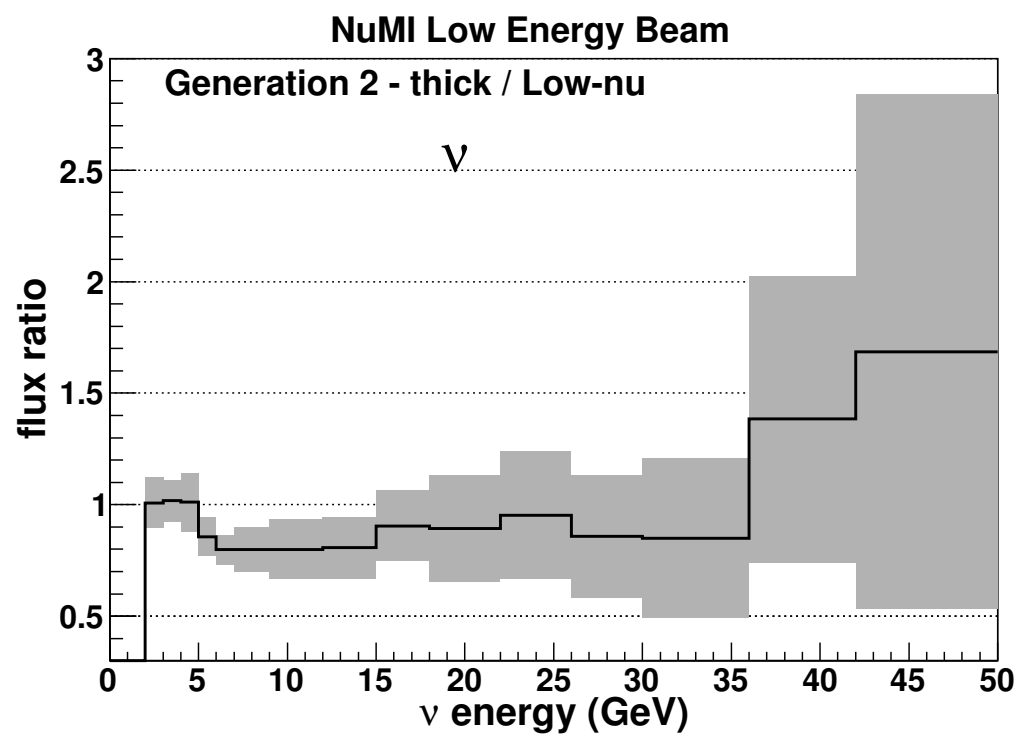

(b) Ratios.

FIG. 6.4: Gen2-thick $\nu_{\mu}$ flux vs low-nu. 


\begin{tabular}{|c|c|c|}
\hline Comparison & $2-50 \mathrm{GeV}$ & $2-22 \mathrm{GeV}$ \\
\hline & $\chi^{2} / N D F$ & $\chi^{2} / N D F$ \\
\hline using full covariance matrix & & \\
$G^{\text {thin }}-L$ & $7.3 / 15$ & $4.8 / 10$ \\
$G^{\text {thick }}-L$ & $61.3 / 15$ & $18.6 / 10$ \\
\hline using diagonal elements only & & \\
$G^{\text {thin }}-L$ & $8.2 / 15$ & $5.3 / 10$ \\
$G^{\text {thick }}-L$ & $68.5 / 15$ & $38.3 / 10$ \\
\hline
\end{tabular}

TABLE 6.2: $\chi 2 / N D F$ for Generation 2 and low-nu difference. Values taken from [92].

thick and Gen2-thin adopting the low-nu binning described in Table 6.1. The $\chi^{2}$ test of the hypothesis $G^{\text {thick }}-G^{\text {thin }}=0$ has been calculated. Four $0.5 \mathrm{GeV}$ size bins have been added to cover energies less than $2 \mathrm{GeV}$. The results are shown in Table 6.3. The agreement between both fluxes is poor in all cases. However, this result strongly depends on the correlation assumptions we have made when incorporating the data. One possible solution would be to study the effect of different correlation assumptions in order to find a way to "reconcile" both flux predictions. This work can be done especially in Gen2-thin, where we assume 100\% bin-tobin correlation for NA49 data. Given that some of the data uncertainties, like the normalization, have definitive positive correlation and the big discrepancy in the $\chi^{2}$ study, reconciling both predictions, Gen2-thick and Gen2-thin, would have to take not well-founded assumptions, like strong negative bin-to-bin correlations for some systematics. For these reasons we consider Gen2-thin and Gen2-thick to be in disagreement.

Summing up this section: Generation 2 gives us the chance to have two flux predictions. Nevertheless, the studies presented suggest that the two predictions are in disagreement each other, and Gen2-thin is in agreement with the low-nu flux results while Gen2-thick is not. Therefore, Gen2-thin will be used as the flux 


\begin{tabular}{|c|c|c|c|}
\hline & $0-50 \mathrm{GeV}$ & $2-50 \mathrm{GeV}$ & $2-22 \mathrm{GeV}$ \\
\hline & $\chi^{2} / N D F$ & $\chi^{2} / N D F$ & $\chi^{2} / N D F$ \\
\hline full covariance matrix & & & \\
& $144.7 / 19$ & $91.0 / 15$ & $76.1 / 10$ \\
\hline diagonal elements only & & & \\
& $62.1 / 19$ & $25.4 / 15$ & $22.0 / 10$ \\
\hline
\end{tabular}

TABLE 6.3: $\chi 2 / N D F$ for Gen2-thick and Gen2-thin difference. Values taken from $[92]$.

determined by this thesis.

\subsection{Additional constraint using neutrino electron scattering events}

An additional constraint based on the neutrino-electron scattering events can be applied to the flux, on top of the hadron production corrections. As was mentioned in Section 3.4, the cross-section for this reaction is well determined by the Standard Model since all participants are leptons.

Figure 6.5 shows the energy distribution of the neutrino-electron elastic scattering candidates interacting in the MINERvA tracker. In total, MINERvA isolated $135 \pm 17(\sim 12.6 \%)$ candidates after the background subtraction and efficiency correction. These events were collected using $3.43 \times 10^{20}$ POT in LE010z185i.

We implement the $\nu e \rightarrow \nu e$ constraint by comparing to the predicted number of these events from the a priori flux. Then, we use this comparison to weight up or down the more likely or unlikely universes by computing the likelihood $\mathcal{L}_{i}$ for each universe i: 


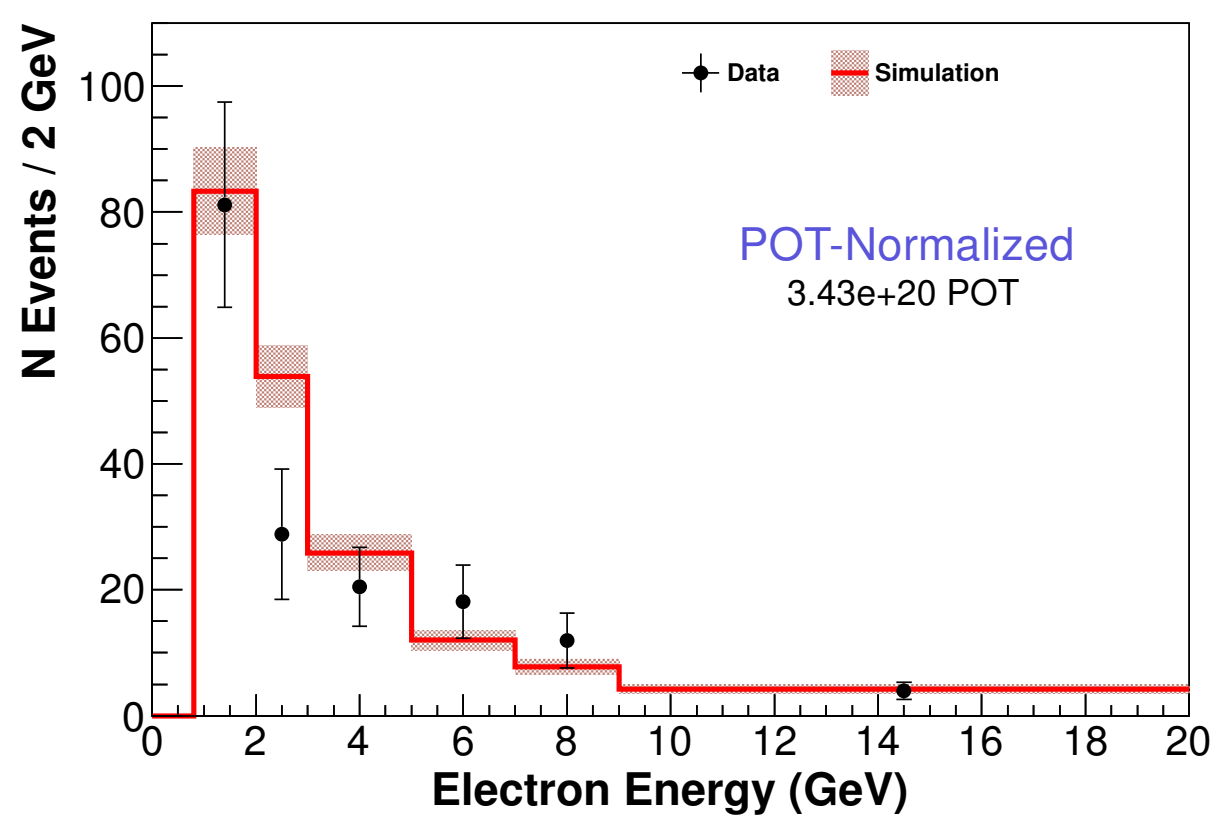

FIG. 6.5: Electron energy distribution from $\nu$-e scattering measured by MINERvA after the background subtraction and efficiency correction (Plot taken from [93]. 


$$
\mathcal{L}_{i}\left(\vec{N}_{\nu e \rightarrow \nu e} \mid \vec{Q}\right)=\frac{1}{(2 \pi)^{M / 2} \operatorname{det}(V)^{1 / 2}} \exp \left(-\frac{1}{2}(\vec{N}-\vec{Q}) \cdot \mathbf{V}_{N}^{-1} \cdot(\vec{N}-\vec{Q})\right)
$$

where $\mathrm{M}$ is the number of data bins. $\vec{N}$ and $\vec{Q}$ are vectors of the measured number and the predicted number, respectively. Every entry in those vectors is the corresponding bin content. $\mathbf{V}$ is the data covariance matrix. These likelihoods are stored and can be used as weights that multiply any quantity that varies with the flux, particularly, the flux itself.

Figure 6.6 shows how the predicted distribution of $\nu e \rightarrow \nu e$ from the a priori Generation 1 flux changes after applying this constraint. Every entry in the a priori plot (black line) is the corresponding value in a universe $i$ and, it is multiplied by $\mathcal{L}_{i}$ and renormalized to preserve the original number of universes (red line). The mean shift and the uncertainty is reduced significantly (more than $30 \%$ ).

The weights from this constraint have been calculated and applied to Generation 2 in MINERvA [94]. Figure 6.7a shows the effect on the $\nu_{\mu}$ Gen2-thin flux. The black lines represent the a priori Gen2-thin flux and the red line the effect of appying the $\nu-e$ constraint. This produces a reduction of the flux peak by approximately $2 \%$ and a dip of $4 \%$ reduction in the falling edge of the flux. The fractional uncertainty also reduces as can be seen in Figure 6.7b.

A measurement of $\nu_{e}$ quasi-elastic and quasi-elastic-like scattering on hydrocarbons was recently published by MINERvA using this procedure on the Generation 1 flux [95]. All future MINERvA publications will likely include this additional constraint. 


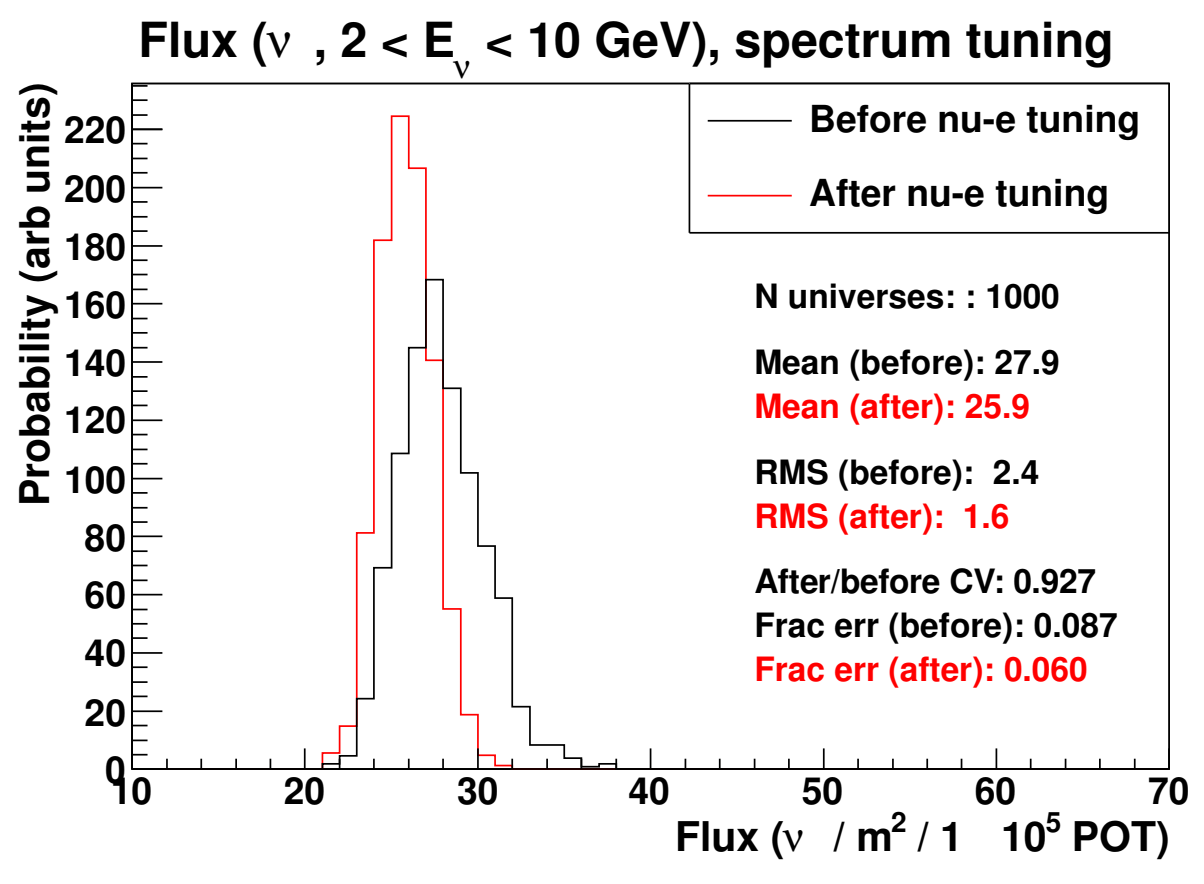

FIG. 6.6: Predicted $\nu e \rightarrow \nu e$ events from the a priori flux before and after the constraint (taken from [93]). 


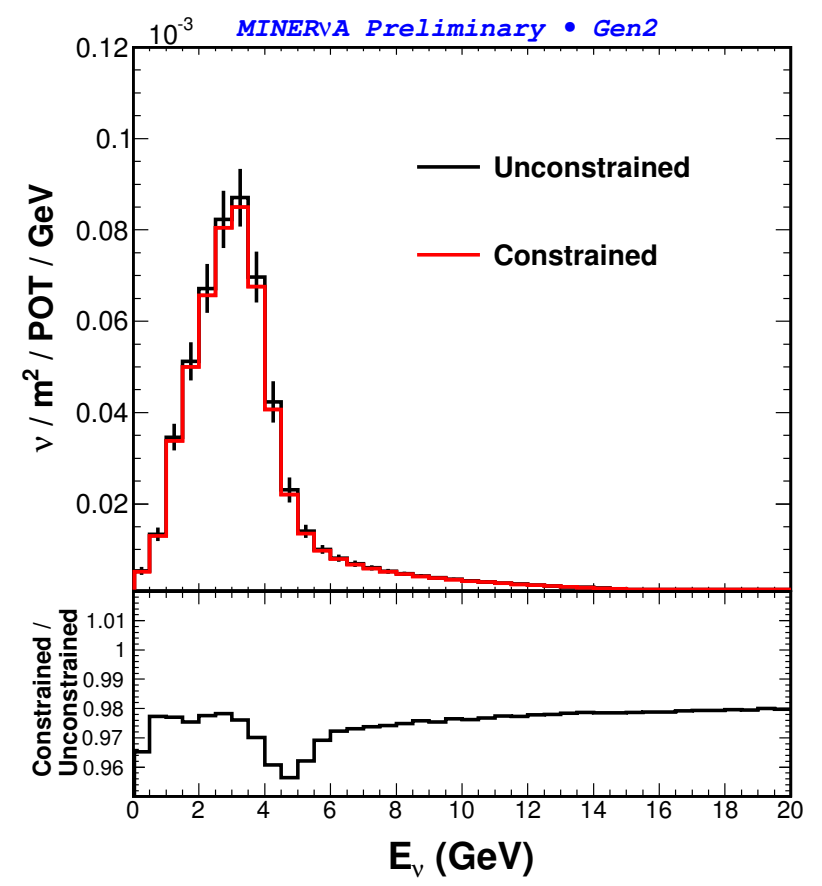

(a) Flux change

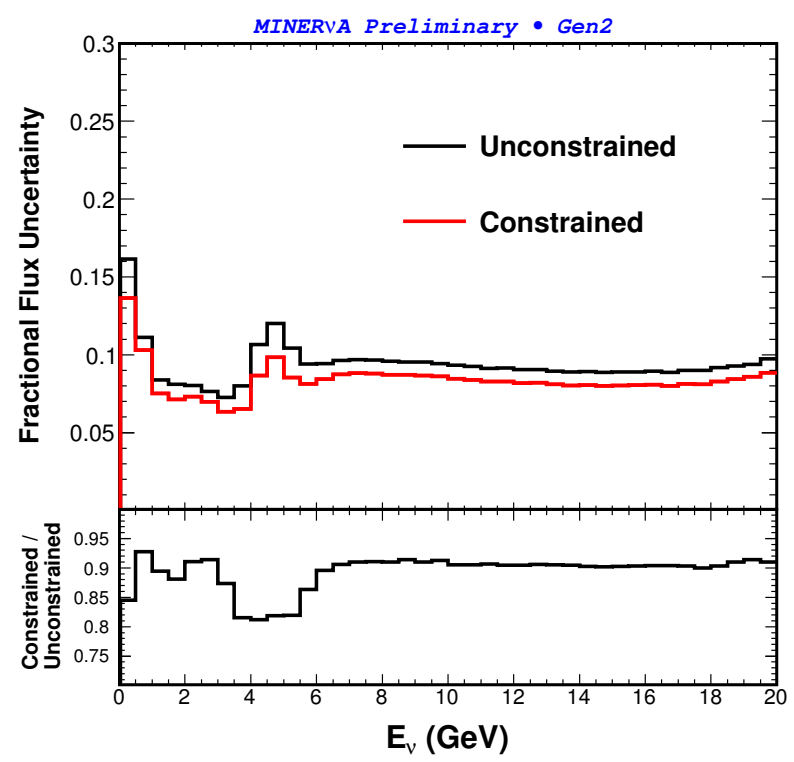

(b) Fractional uncertainty change

FIG. 6.7: Effect of the $\nu e$ constraint on the Gen2-thin flux. Top: the flux spectrum. Bottom: the fractional uncertainties. The black like comes from the a priori flux and the red line is after appying the $\nu-e$ constraint. Plots taken from [94]. 


\subsection{Comparison of Gen2-thick with Generation 0 and Generation 1}

All MINERvA analyses (Table 5.1) and results already published by MINERvA can be updated to the standard flux version, Gen2-thin. This section presents comparisons between earlier versions of the flux. This provides an idea of how the MINERvA cross-section results would change using Gen2-thin. However, other studies needs to be considered in the updating process such us the effect of the backgrounds when switching flux versions.

This is the list of flux comparisons presented in this sections:

- $\nu_{\mu}$ flux Gen2-thin vs Generation 0 (Figure 6.8).

- $\bar{\nu}_{\mu}$ flux Gen2-thin vs Generation 0 (Figure 6.9).

- $\nu_{\mu}$ flux Gen2-thin vs Generation 1 (Figure 6.10).

- $\bar{\nu}_{\mu}$ flux Gen2-thin vs Generation 1 (Figure 6.11).

- $\nu_{e}$ flux Gen2-thin vs Generation 1 (Figure 6.12).

- $\nu_{\mu}$ flux Gen2-thin vs Generation 1 + neutrino electron scattering constraint (Figure 6.13).

- $\nu_{e}$ flux Gen2-thin vs Generation 1 + neutrino electron scattering constraint (Figure 6.14).

- The flux integrated ratios for $\nu_{\mu}, \bar{\nu}_{\mu}$ and $\nu_{e}$ in the 0-20 energy range (Table 6.4). 


\begin{tabular}{|c|c|c|c|}
\hline Neutrino & $\frac{\text { Gen2-thin }}{\text { Gen0 }}$ & $\frac{\text { Gen2-thin }}{\text { Gen1 }}$ & $\frac{\text { Gen2-thin }}{\text { Gen1+nu-e }}$ \\
\hline$\nu_{\mu}$ & 0.846 & 0.890 & 0.959 \\
$\bar{\nu}_{\mu}$ & 0.838 & 0.892 & - \\
$\nu_{e}$ & - & 0.900 & 9.970 \\
\hline
\end{tabular}

TABLE 6.4: Integrated flux ratios between generations in the 0-20 energy range. We abbreviate Generation 0 by Gen0, Generation 1 by Gen1 and neutrino electron constraint by nu-e.

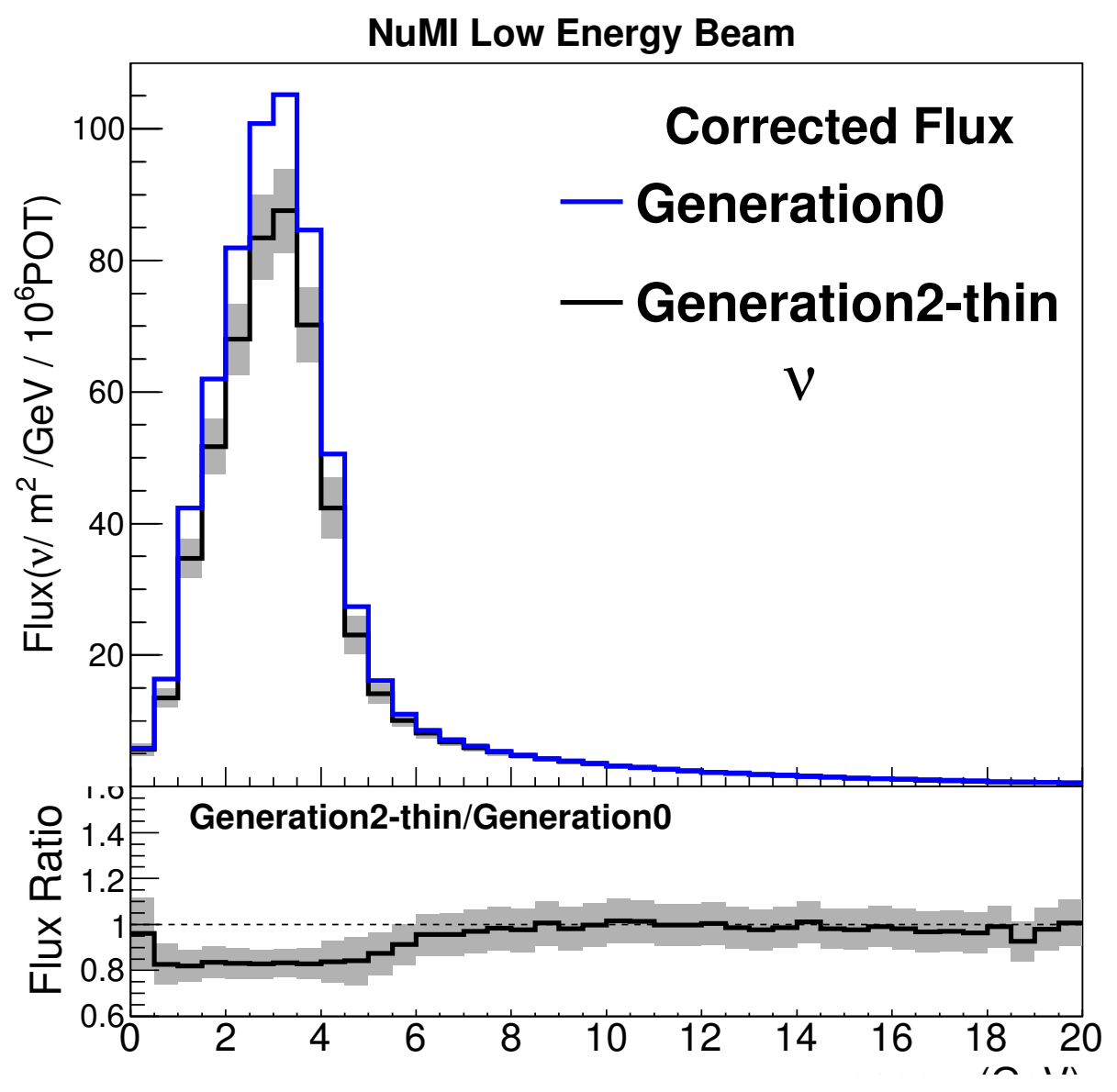

FIG. 6.8: $\nu_{\mu}$ flux Gen2-thin vs Generation 0. 


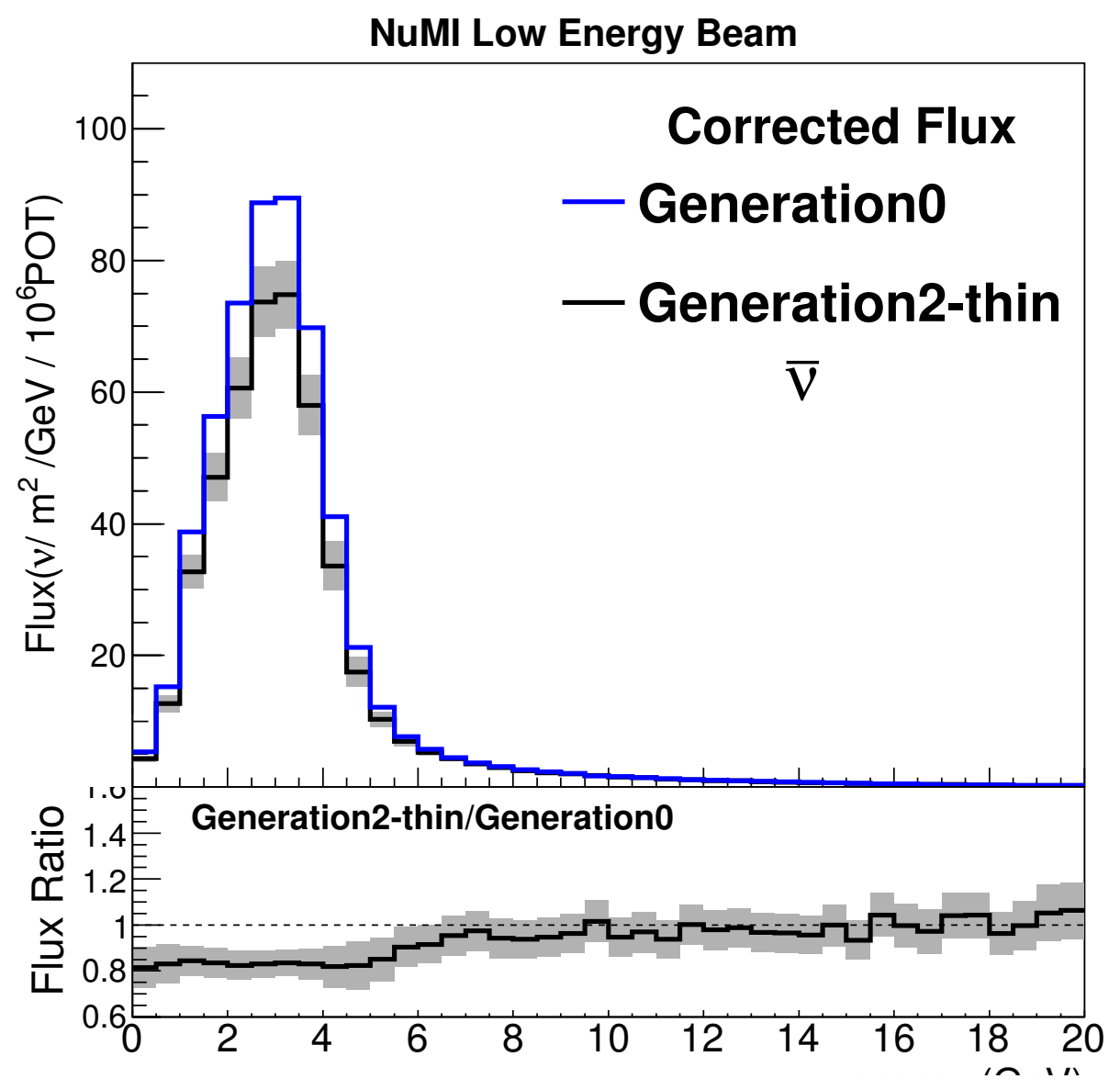

FIG. 6.9: $\bar{\nu}_{\mu}$ flux Gen2-thin vs Generation 0. 


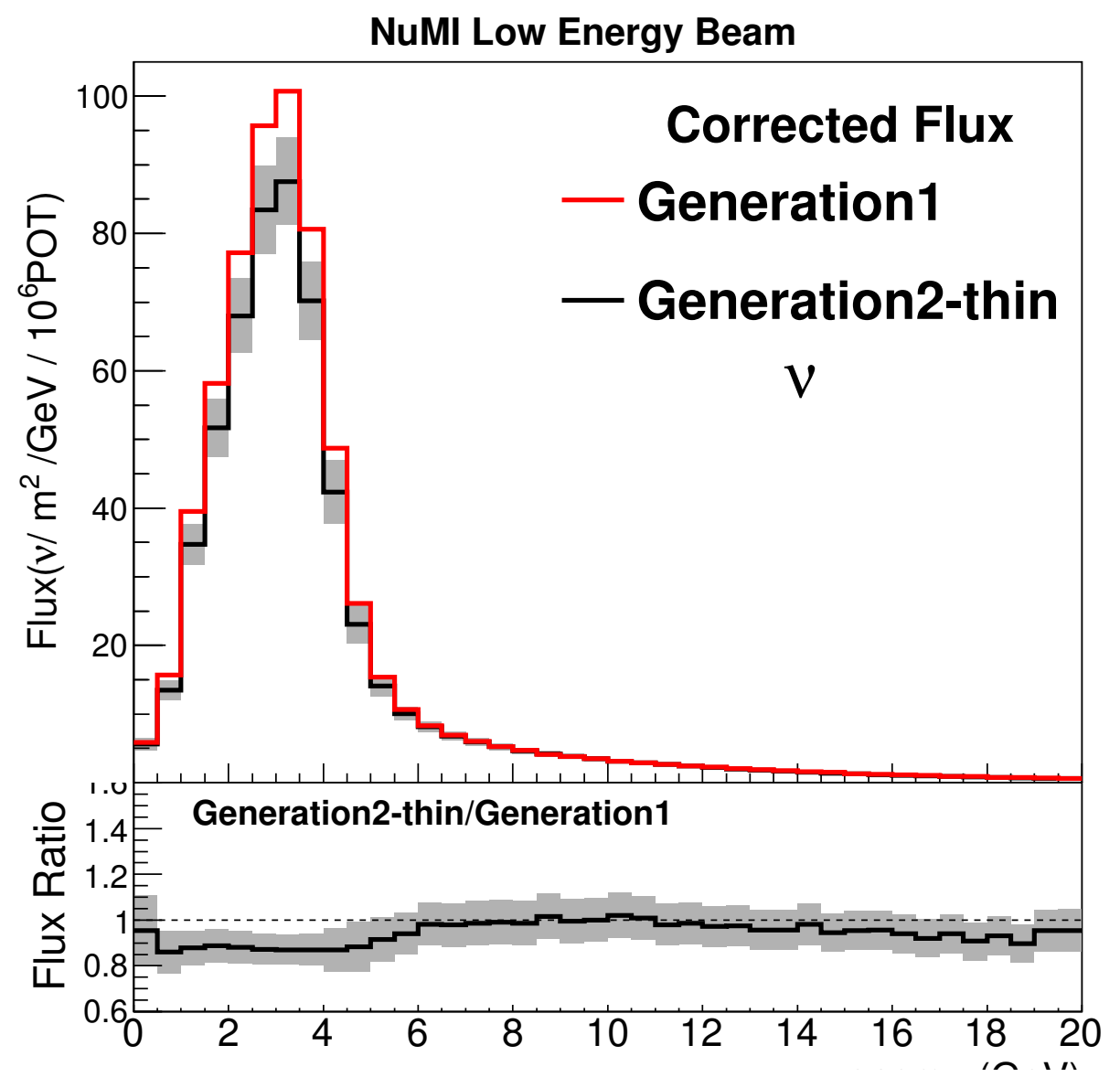

FIG. 6.10: $\nu_{\mu}$ flux Gen2-thin vs Generation 1. 


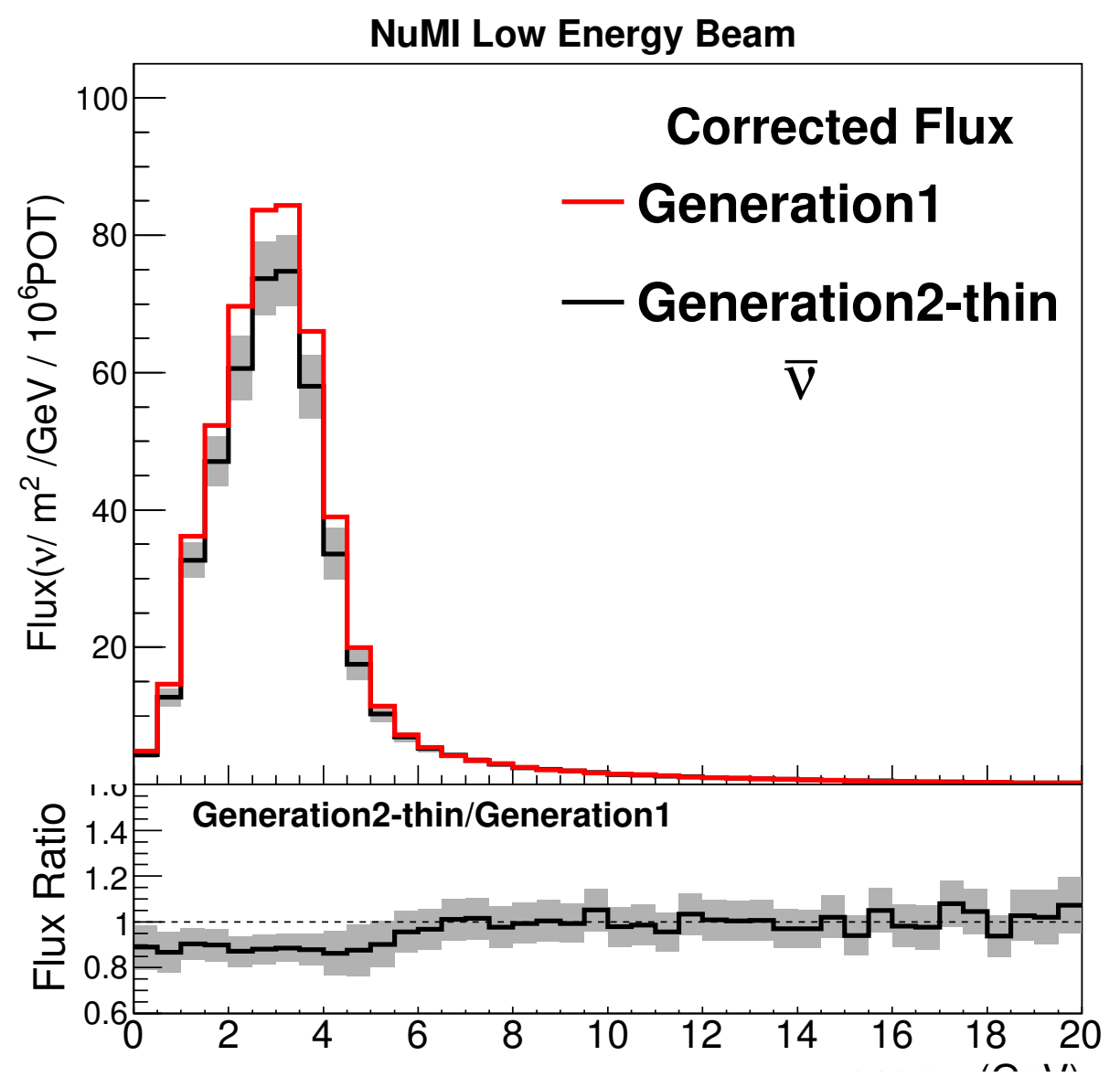

FIG. 6.11: $\bar{\nu}_{\mu}$ flux Gen2-thin vs Generation 1. 


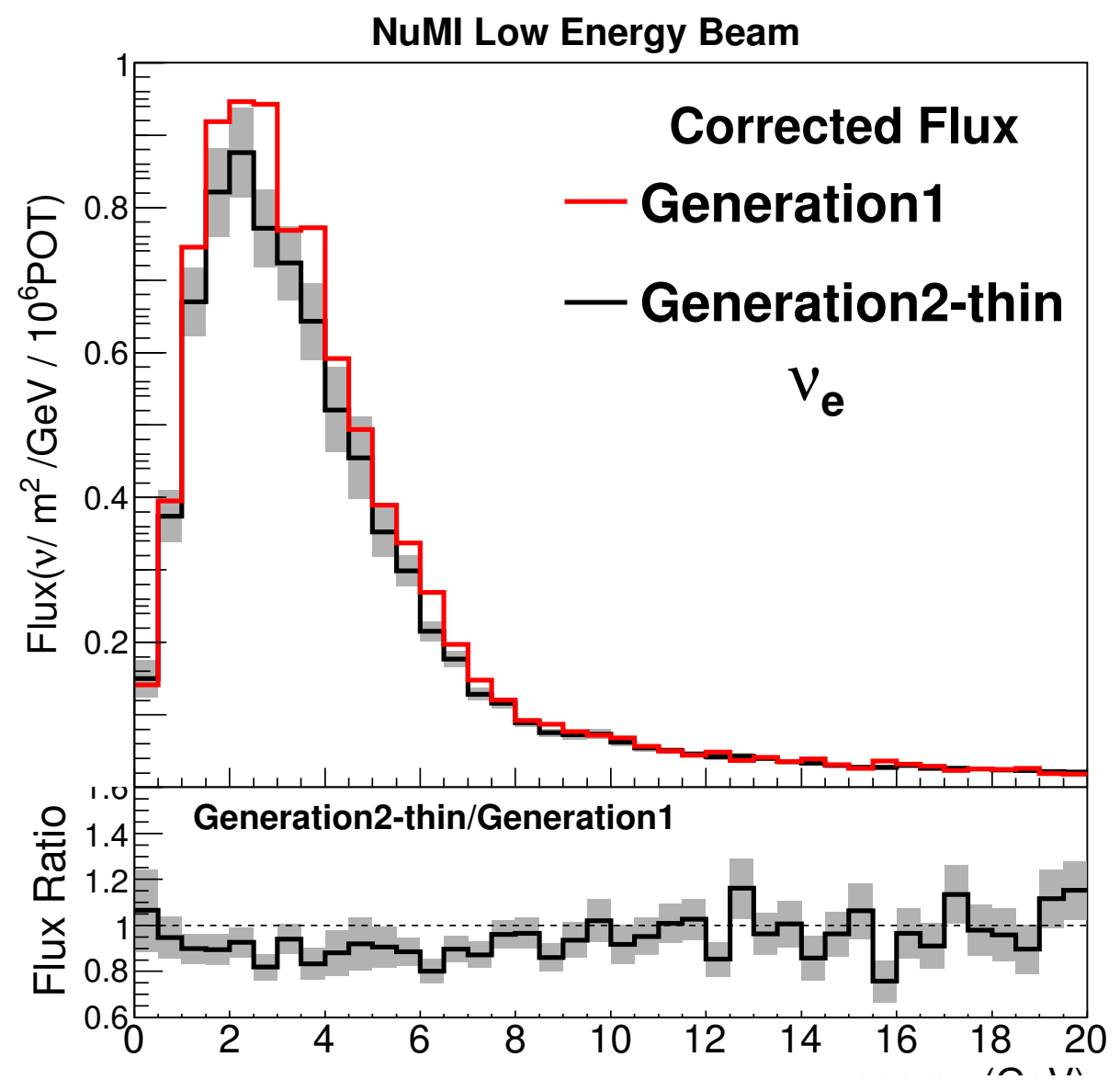

FIG. 6.12: $\nu_{e}$ flux Gen2-thin vs Generation 1. 


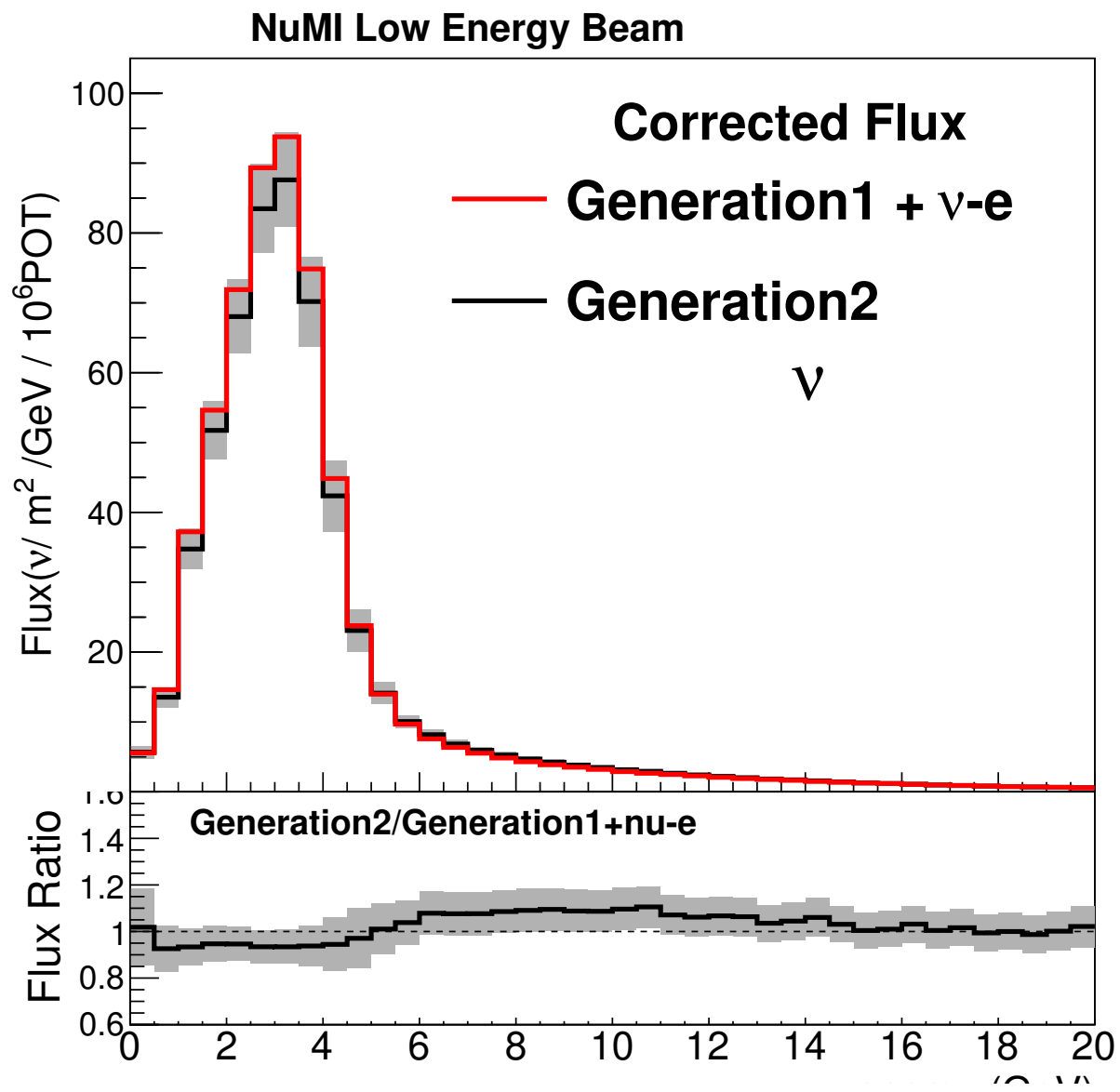

FIG. 6.13: $\nu_{\mu}$ flux Gen2-thin vs Generation $1+$ neutrino electron scattering constraint. 


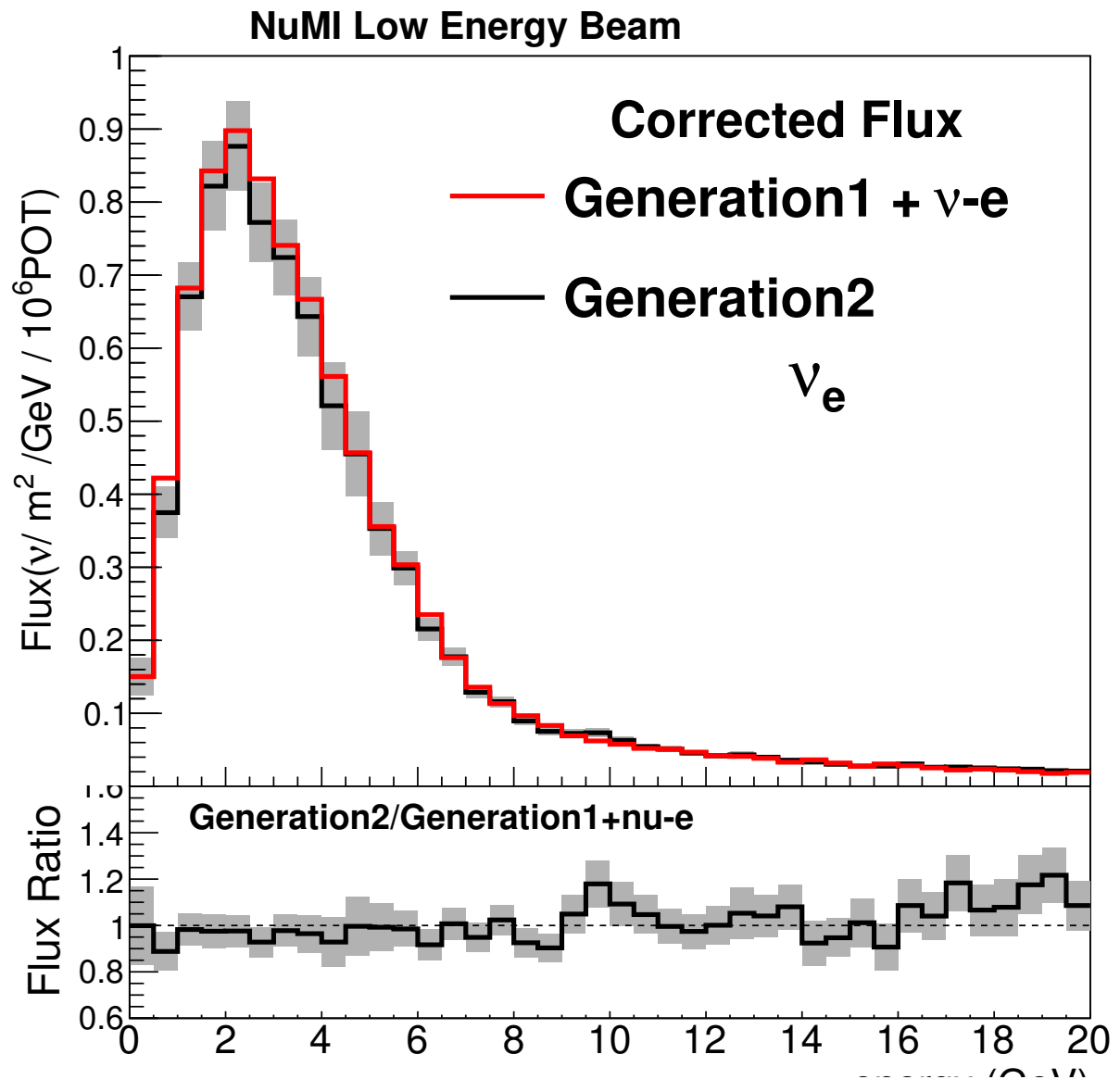

FIG. 6.14: $\nu_{e}$ flux Gen2-thin vs Generation 1 + neutrino electron scattering constraint. 


\section{CHAPTER 7}

\section{Medium Energy Beam results}

The focus of this thesis has been to determine the a priori LE flux for MINERvA experiment. However, the procedure we followed, as well as the tools we developed, are intended to be directly applicable to calculate the Medium Energy flux for any point in the NuMI beamline. As we mentioned in the introductory chapter (Section 2), experiments like MINERvA, MINOS + and NOvA are currently taking data for the neutrino cross-section and oscillation physics programs at Fermilab and they can benefit from the results of this thesis.

The objective of this chapter is to show our results for the ME flux at MINERvA (first section) and the capability of our techniques to extend to any detector that sees $\nu_{\mu}$ neutrinos (in the second section). We present our flux prediction for NOvA Near Detector as an example. For the results shown in this chapter, we used Gen2thin flux version as it was determined that has better physics consistency than Gen2-thick (see Sections 5.4 and 6.1.1). 


\subsection{Gen2-thin flux for ME MINERvA}

Most of the discussion of the LE flux at MINERvA can be applied to understand the results when we switch to the ME beam mode. In this section, we will emphasize only those that are particular to the ME mode. The simulated ME flux spectrum at MINERvA (before any HP constraint) was shown in Figure 2.14 with its characteristic peak at $6 \mathrm{GeV}$. The key differences in $\mathrm{ME}$ with respect to the LE mode comes from the changes in the relative positions between the target and horn (it was enlarged), between horns (it was enlarged) and the length of the target ( $\sim 20 \%$ longer) as was described in Section 2.3. These changes cause the focusing system to enhance higher energy mesons which then decay to give higher energy neutrinos (for instance $\pi^{+}$yields shown in the lower right side of Figure 2.13). See Section A.1 for details of the focusing components.

Figure $7.1 \mathrm{a}$ shows the average number of hadronic interactions per $\nu_{\mu}$ that passes through the center of the MINERvA tracker, the total number as a black line and those that are handled by every PPFX reweighter listed in Table 4.4. The shapes of every line are similar to the LE configuration (Figure 5.4), however, there are more interactions in total while every reweighter has more interactions to handle. This is because the ME target is longer than the LE target so more interactions happen inside the target. Pions and kaons produced in proton - carbon interactions increase, but also incident nucleons not handled directly by data decrease since fewer protons leave the target.

The average material traversed by particle beams in the relevant NuMI volumes per $\nu_{\mu}$ is shown in 7.1a. It also has the same trend as LE (Figure 5.7a) but we have more contributions from the primary proton beam passing through the target. Pion attenuation in the target becomes dominant in the falling edge of the focusing peak and in general, primary protons, pions, and kaons traverse more material than in 
LE.

Figure 7.2 shows the flux spectrum for $\nu_{\mu}$ in Medium Energy integrated over the MINERvA fiducial volume (see the introduction to Chapter 5) in the energy region 0-20 GeV with the error bars corresponding to the HP uncertainties. At the moment this thesis is being written, the ME focusing uncertainties are being revisited. For this reason, we use the focusing uncertainties corresponding to the LE100z200i plus the horn inner conductor uncertainty (Section 2.5.5) calculated for $\nu_{\mu}$. Nevertheless, we expect that this is a good approximation for the final ME focusing uncertainties. A table of the ME flux is shown in Table B.3 for all neutrino types.

The $\nu_{\mu}$ hadron production fractional uncertainty is shown in Figure 7.3. It has a similar shape but is slightly larger than LE as we can see when we compare with Figure 5.14a. However, as we expect, the absorption and incident meson uncertainties are larger.

The correlation between energy bins is shown in Figure 7.4 for $\nu_{\mu}$ and $\nu_{e}$. Only the hadron production uncertainties are considered. The bins are highly correlated due to our assumption about the bin-to-bin correlation of the thin target data systematic uncertainties.

Table 7.1 contains the $\nu_{\mu}, \bar{\nu}_{\mu}$ and $\nu_{e}$ fluxes integrated over the $0-20 \mathrm{GeV}$ region along with the hadron production uncertainties.

\begin{tabular}{|l|c|c|}
\hline & integral $\left(\nu / m^{2} / 10^{6} \mathrm{POT}\right)$ & Uncertainty $(\%)$ \\
\hline Gen2-thin $\nu_{\mu}$ & 704.6 & 7.26 \\
\hline Gen2-thin $\bar{\nu}_{\mu}$ & 0.39 & 10.98 \\
\hline Gen2-thin $\nu_{e}$ & 6.90 & 6.76 \\
\hline \hline
\end{tabular}

TABLE 7.1: $\nu_{\mu}, \bar{\nu}_{\mu}$, and $\nu_{e}$ Gen2-thin integrated fluxes in 0-20 GeV. 


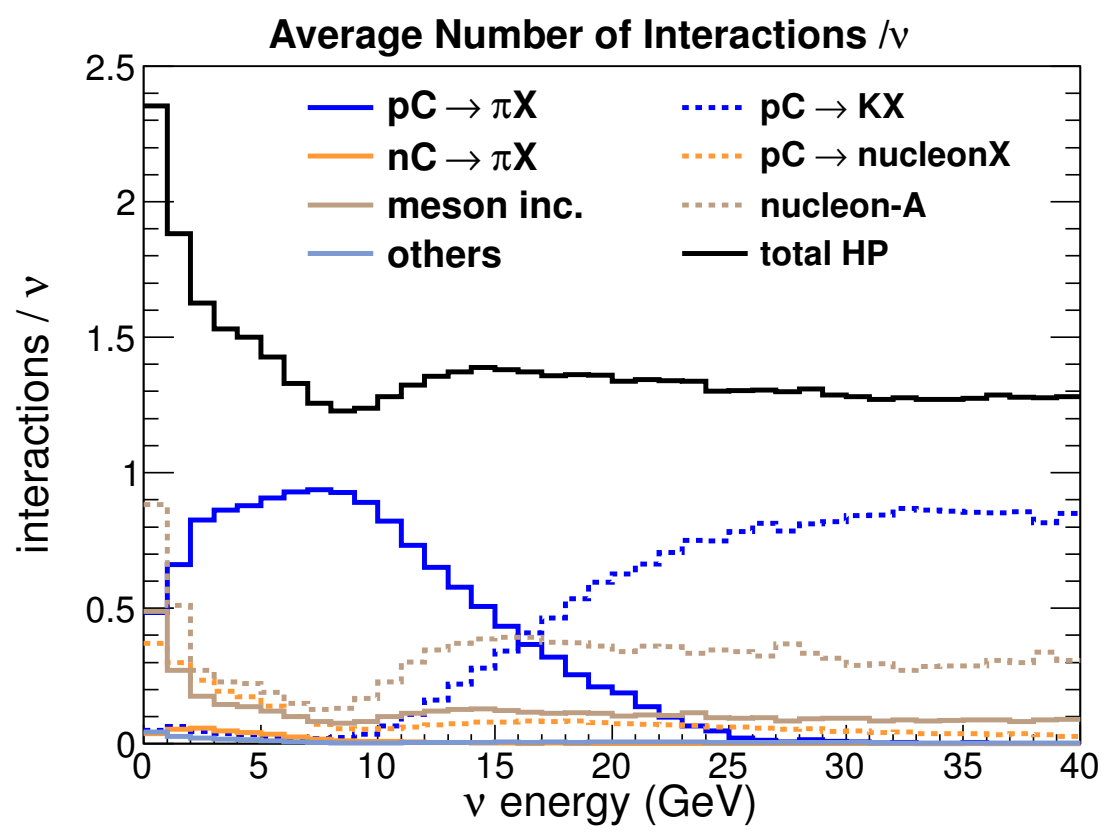

(a) Average number of interactions.

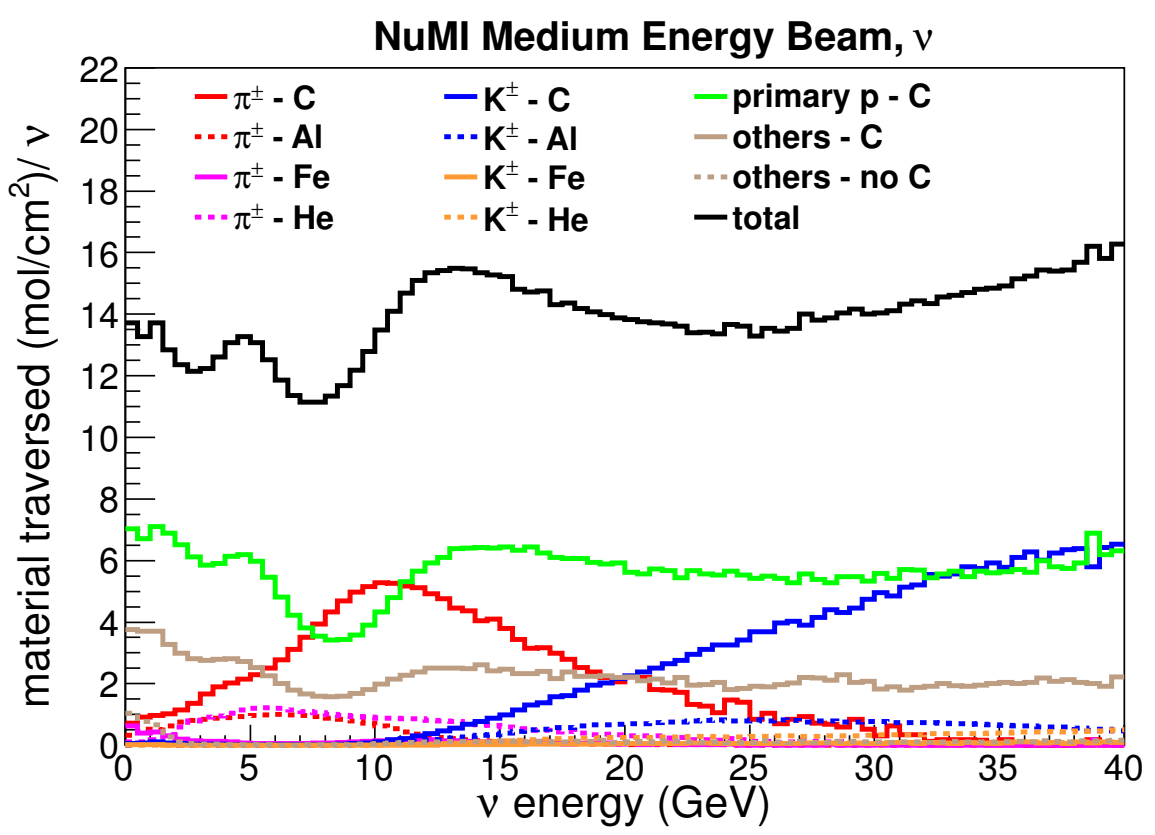

(b) Material traversed.

FIG. 7.1: ME PPFX coverage for $\nu_{\mu}$ in MINERvA. 


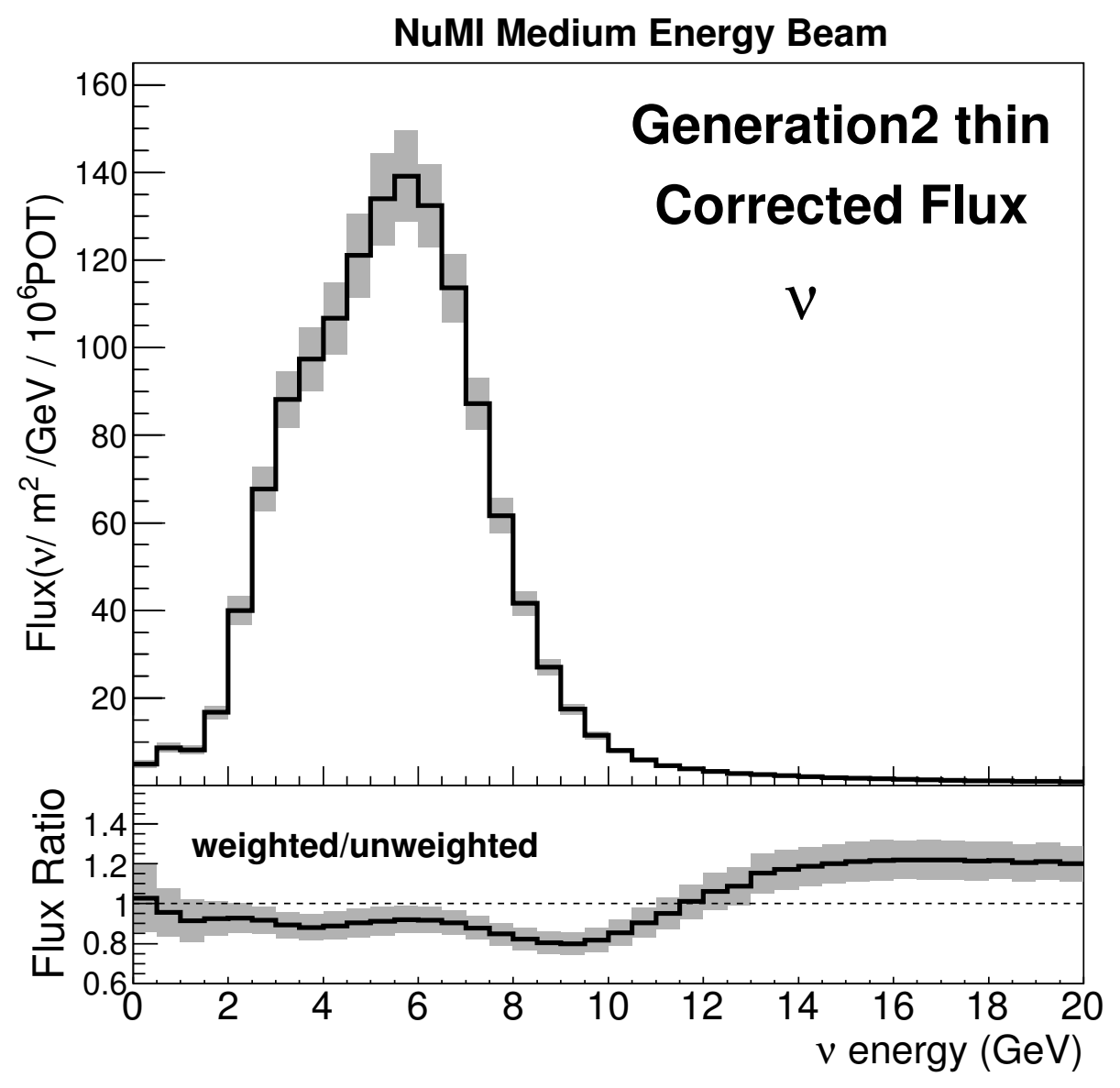

FIG. 7.2: Medium Energy Gen2 - thin $\nu_{\mu}$ flux at MINERvA. 


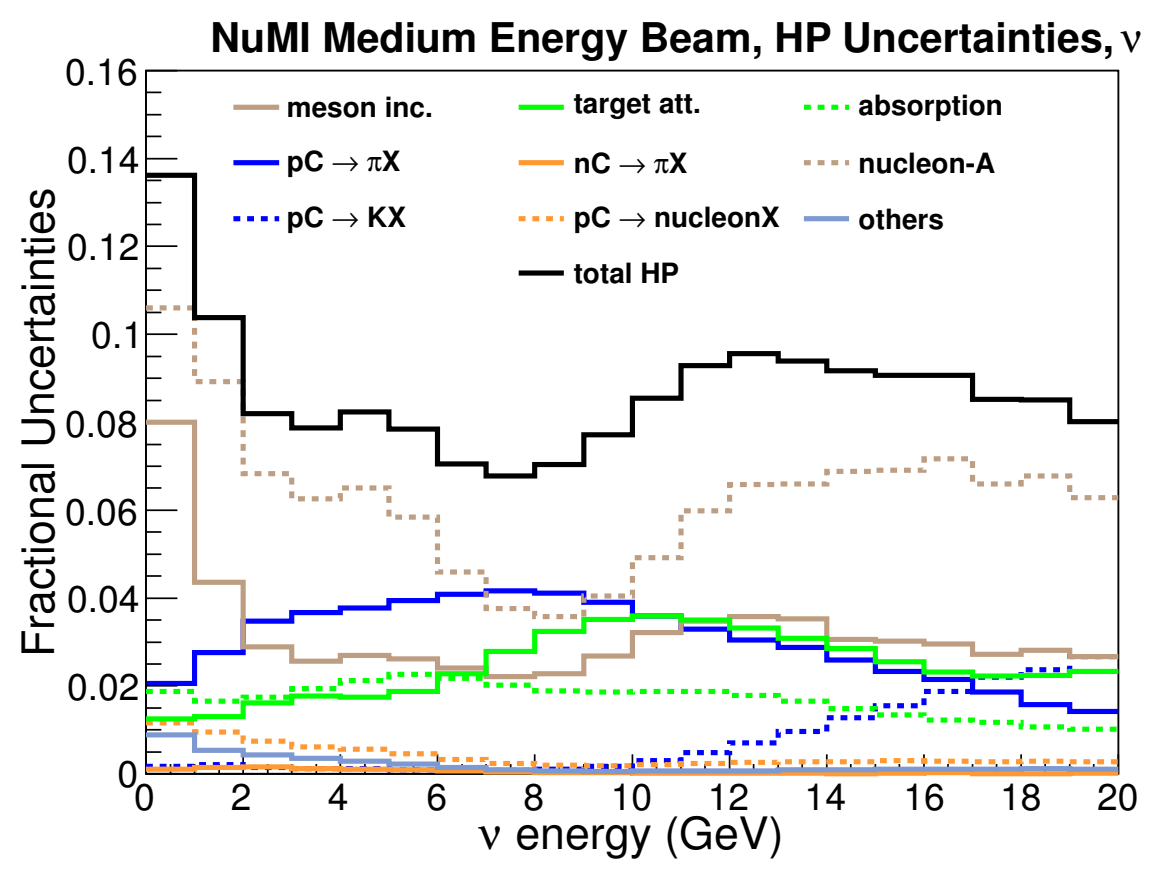

FIG. 7.3: Fractional Uncertainties for ME Gen2-thin $\nu_{\mu}$ flux in MINERvA. 


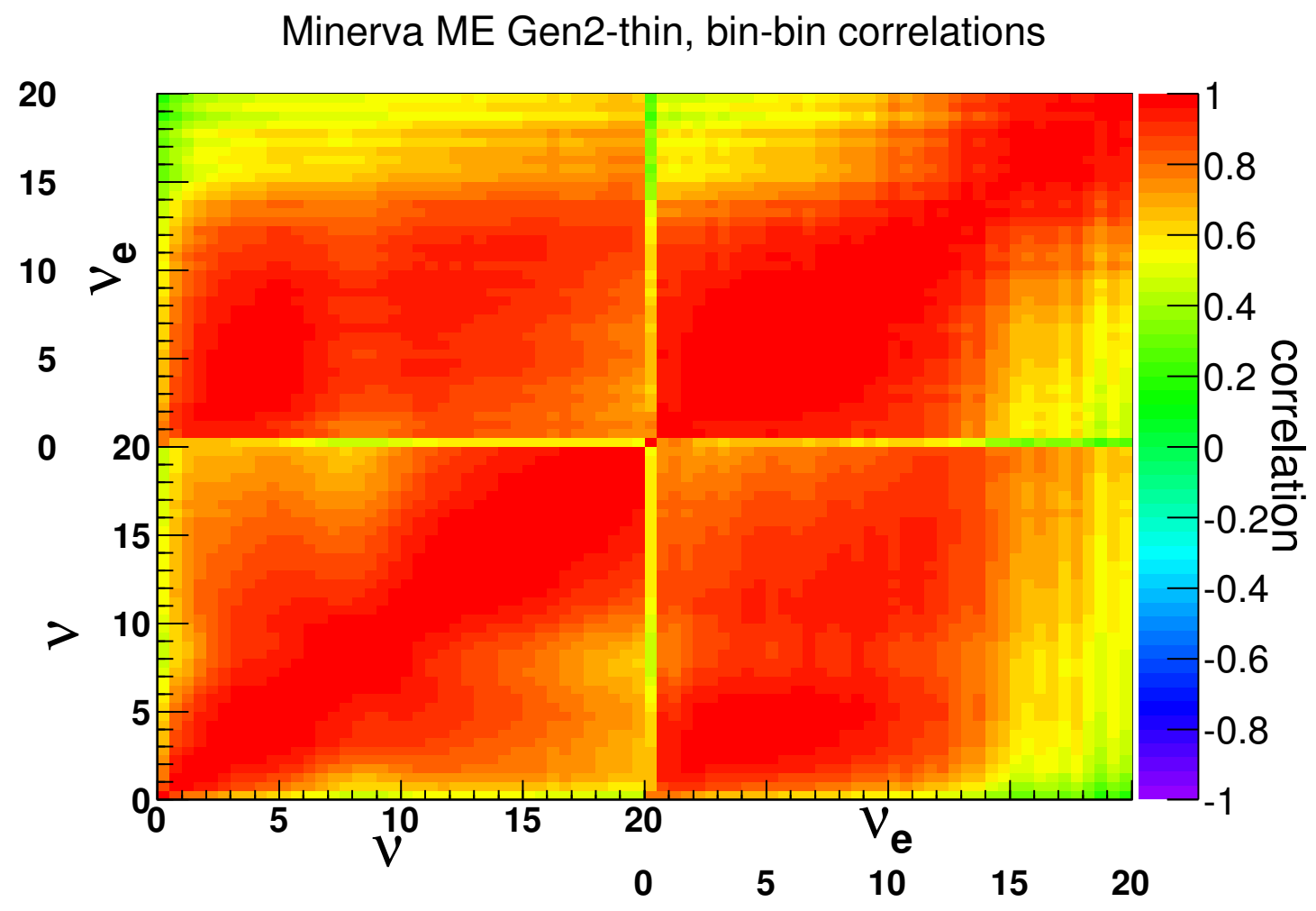

FIG. 7.4: Bin to bin correlation of the ME Gen2-thin $\nu_{\mu}$ and $\nu_{e}$ fluxes at MINERvA. 


\subsection{Gen2-thin for NOvA ND}

As an example of the extension of our procedure to other NuMI detectors, we show the $\nu_{\mu}$ Gen2-thin flux calculated at the center of the NOvA Near Detector's front face. Since the flux spectrum peaks at $2 \mathrm{GeV}$, we restricted the plots shown in this section to the $0-6 \mathrm{GeV}$ energy region.

Figure $7.5 \mathrm{a}$ shows that the average number of hadronic interactions per neutrino is higher than the ME beam at MINERvA due to the off-axis position of the NOvA Near Detector. Most of the interactions are handled by the thin target data, particularly pion production from proton - carbon interactions. However, other interactions become relevant at low energies $(<0.8 \mathrm{GeV})$, particularly nucleons that interact outside of the target that are not covered by data. Figure $7.5 \mathrm{~b}$ shows the material traversed by the particles in the beam. The contribution of mesons is almost constant with energy since the effect of the focusing is not as relevant in an off-axis beam. The category "others-C" in the plots is composed mainly of secondary protons.

Figure 7.6 shows the $\nu_{\mu}$ flux spectrum and the error band from the hadron production uncertainties in the $0-6 \mathrm{GeV}$ energy region (Table B.4 contains these values in $0.1 \mathrm{GeV}$ bin size). The ratio plot indicates that our NuMI flux simulation (g4numi) overestimates the neutrino flux at NOvA by $\sim 10 \%$.

As can be seen in Figure 7.7, the hadron production fractional uncertainties are roughly $8 \%$ around the focusing peak. The biggest uncertainties come from incident nucleons interacting outside of the target and incident mesons where we are not applying any data constraint.

An interesting comparison can be made between our flux results and the pre-

dicted spectrum from the NOvA flux simulation. NOvA uses FLUGG2008 [96] that uses FLUKA for hadronic interactions and GEANT4 for the detector geome- 


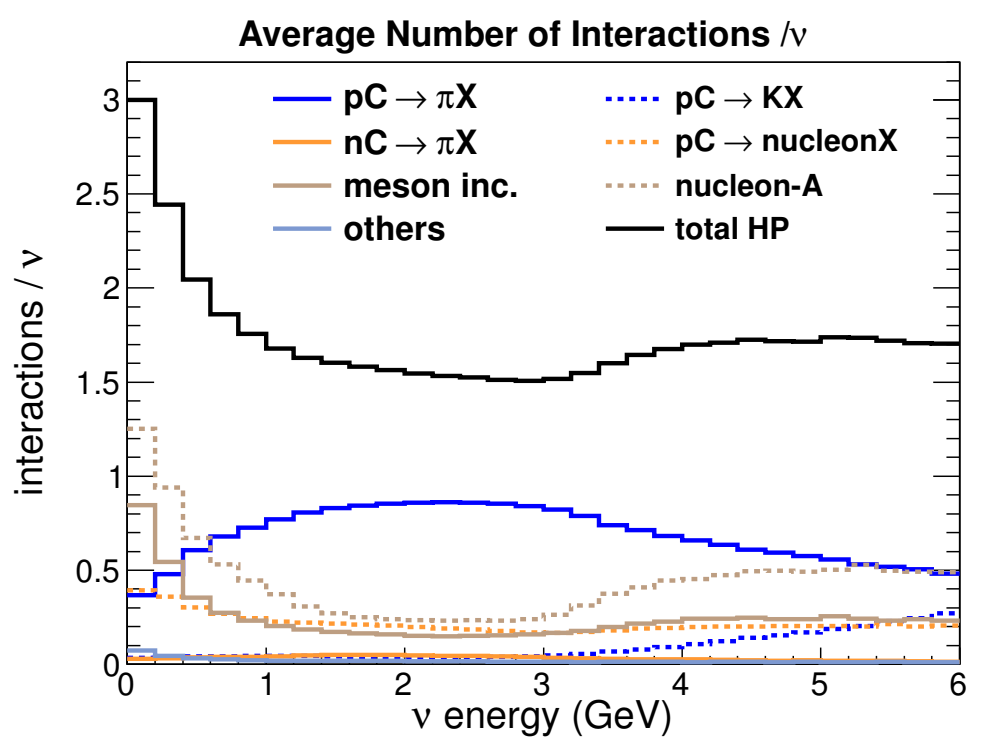

(a) Average number of interactions

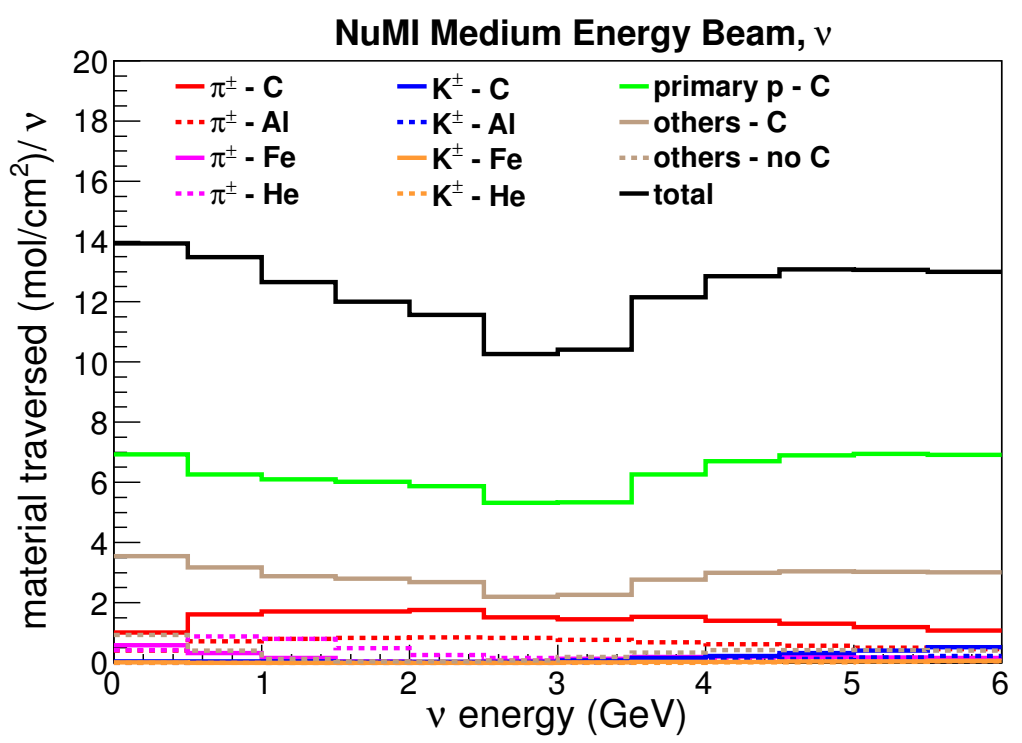

(b) Material traversed

FIG. 7.5: Average number of interactions and material traverse per $\nu_{\mu}$ that pass through NOvA in the ME Gen2-thin flux. 


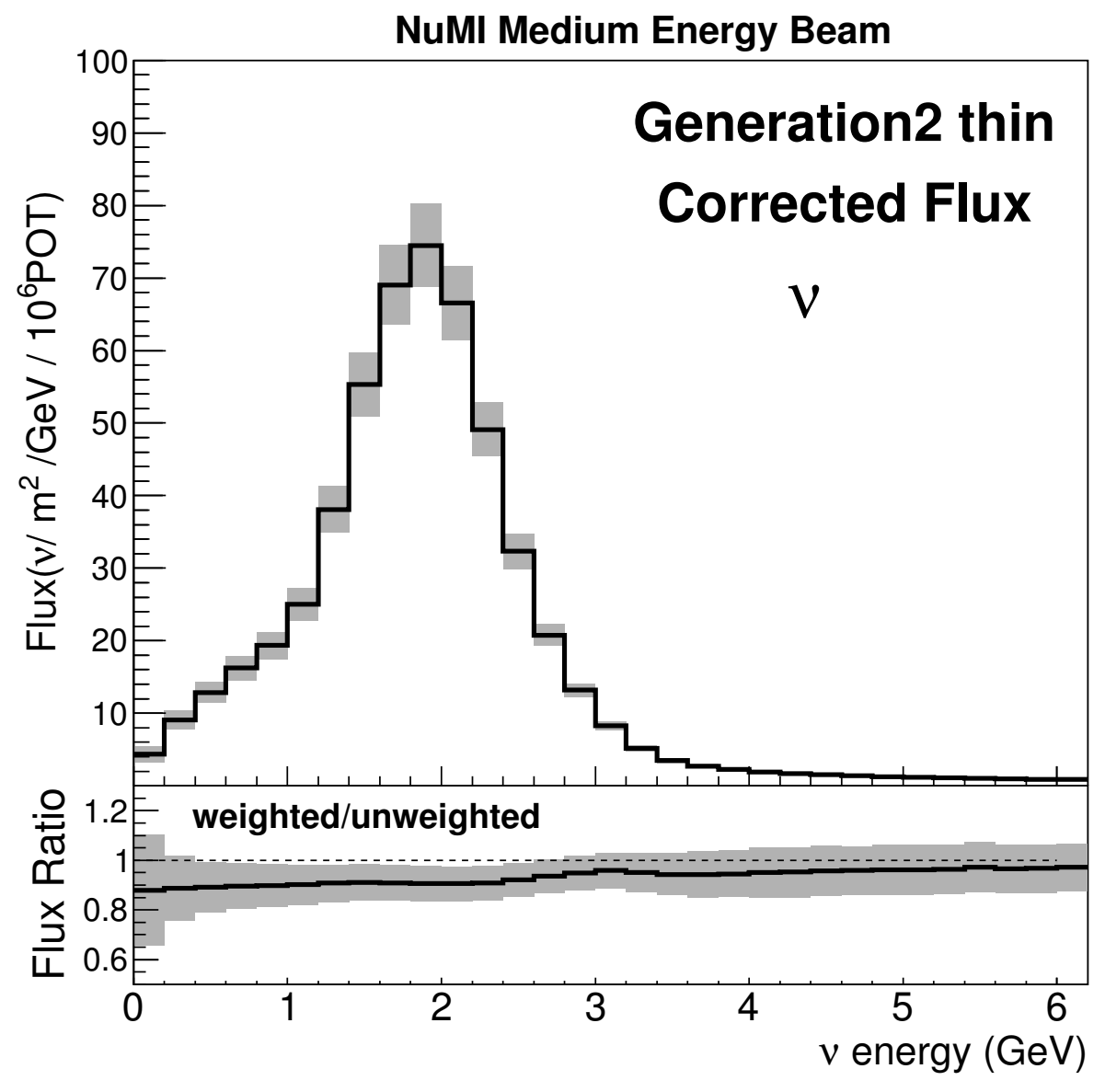

FIG. 7.6: Gen2-thin ME $\nu_{\mu}$ flux at NOvA ND. 


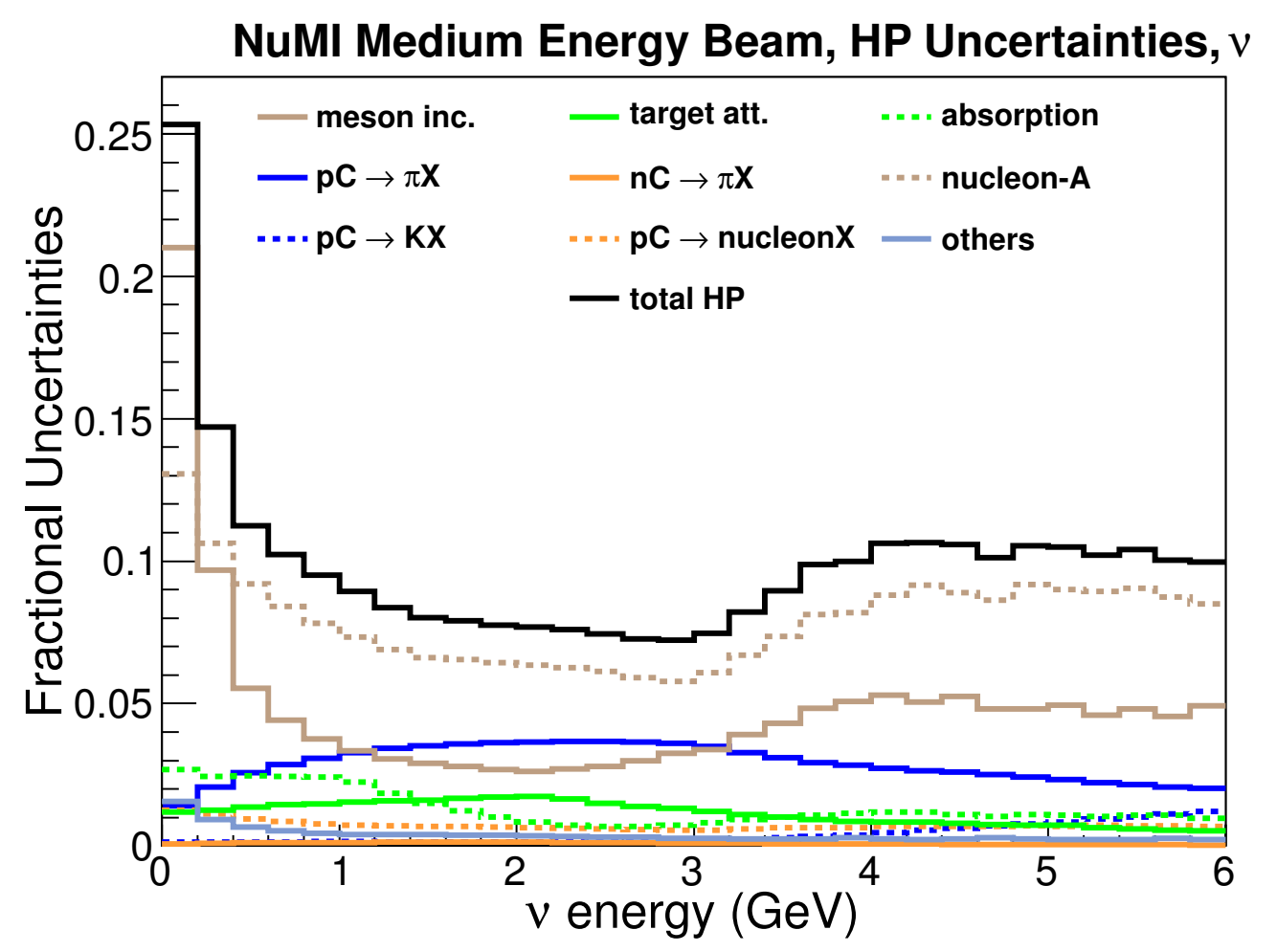

FIG. 7.7: Fractional Uncertainties for ME Gen2 - thin $\nu_{\mu}$ flux in NOvA. 
try and particle tracking. Figure 7.8 shows the ratio between Gen2-thin (called just "ppfx" in the plot) and the FLUKA2008 flux spectra at NOvA's Near Detector (called "flugg" in the plot). The uncertainty on the ratio comes from the uncertainty on the Gen2-thin prediction. Around the focusing peak, we predict 5-6\% fewer neutrinos and a $15 \%$ dip around 2.5 to $4 \mathrm{GeV}$. There are two possible sources that could explain these discrepancies. First, a mismodeling in the FLUKA hadronic interactions since NOvA does not do a hadron production correction. Second, differences in the NuMI geometry incorporated in g4numi with respect to FLUGG2008 could cause the peak to shift, and added material such us the water layer around the Horn 1 inner conductor (Section 2.5.7) could be responsible for attenuating the flux. However, further investigations are needed to give a conclusive explanation.

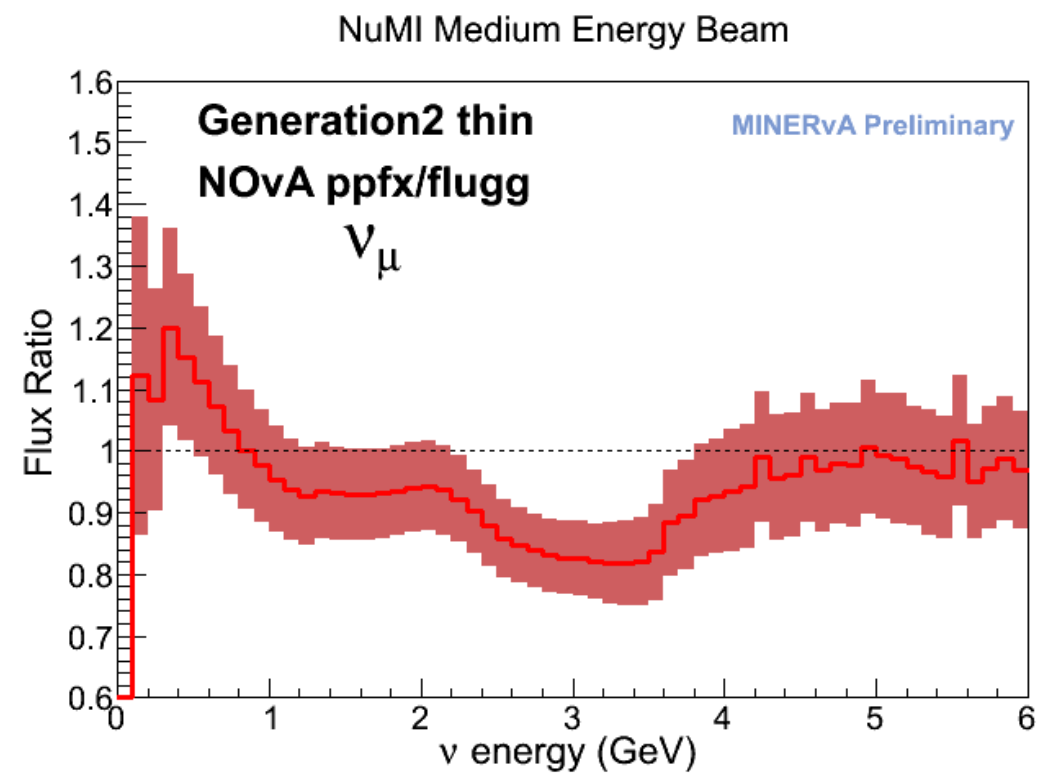

FIG. 7.8: Comparison between Gen2-thin and the FLUGG $\nu_{\mu}$ flux NOvA ND. The error bar comes from the Gen2-thin hadron production uncertainties. 


\section{CHAPTER 8}

\section{Conclusions}

Determining the neutrino flux with small and well-understood uncertainties is crucial for neutrino - nucleus cross-section experiments such as MINERvA and neutrino oscillation experiments such as MINOS and NOvA (Chapter 1). We have shown a new computation of the a priori NuMI flux constrained by all relevant hadron production data (Chapter 4) and using the current knowledge of the NuMI beamline, which reduces the uncertainties and improves error budget accounting.

The MINERvA strategy (Chapter 3) also includes the use of in situ measurements that provide additional information about the flux and can be applied on top of the hadron production constraint. These in situ measurements help us check the consistency of the a priori flux (the low-nu method) and to serve as an additional constraint (neutrino - electron scattering) (Chapter 6).

We calculated two a priori flux versions for the LE beam, Gen2-thin and Gen2-thick. The comparison of these two versions shows an energy dependence disagreement (Chapter 5) for the $\nu_{\mu}$ flux in the LE010z185i configuration. A quantitative study found that the Gen2-thin and low-nu fluxes are in good agreement, whereas the Gen2-thick flux agrees poorly with low-nu (Chapter 6). Based on 
this fact, we presented Gen2-thin as the main flux result of this thesis and recommended using it as standard flux for the current and future MINERvA analyses. We also presented a comparison of the Gen2-thin LE flux to previous flux versions, Generation 0 and Generation 1. We also presented the Gen2-thin ME flux for MINERvA with 7-8\% hadron production uncertainties around $6 \mathrm{GeV}$ (Chapter 7).

All techniques and tools developed in this thesis (Chapter 4) are intended to be available for any other experiment that sees neutrinos from the NuMI beamline. Particularly we introduced a computational tool called PPFX that is open and public. As an example of extending our procedure to other detectors, we presented the flux calculated in the center of the front face of the NOvA Near Detector with 7-8\% hadron production uncertainty around $2 \mathrm{GeV}$. The extension to other beamlines is straightforward.

Our work indicates where additional hadron production data are needed in order to further reduce uncertainties for NuMI and LBNF. Particularly, it would be very valuable to measure the invariant differential cross-sections of:

- Proton interactions on a thin target with materials different than carbon.

- Quasi-elastic proton production.

- Incident meson interactions producing pions.

Our calculation of the Gen2-thick LE flux also shows that the uncertainties can be reduced significantly by using thick target data, such us measurements of particle production on a replica or spare target. In this context, the program of measurements proposed by US-NA61 on thin and thin targets is extremely important. 


\section{APPENDIX A}

\section{Supplementary material for \\ Chapter 2}

\section{A.1 Focusing components}

This section contains:

- NuMI flux focusing components (Figure A.1) in LE010185i at MINERvA.

- Longitudinal vs. transverse momentum yield per $\pi^{+} \nu_{\mu}$ parent:

- Unfocused (Figure A.2).

- Horn2 only (Figure A.3).

- Horn1 only (Figure A.3).

- Underfocused (Figure A.5).

- Overfocused (Figure A.6).

- Others (Figure A.7). 

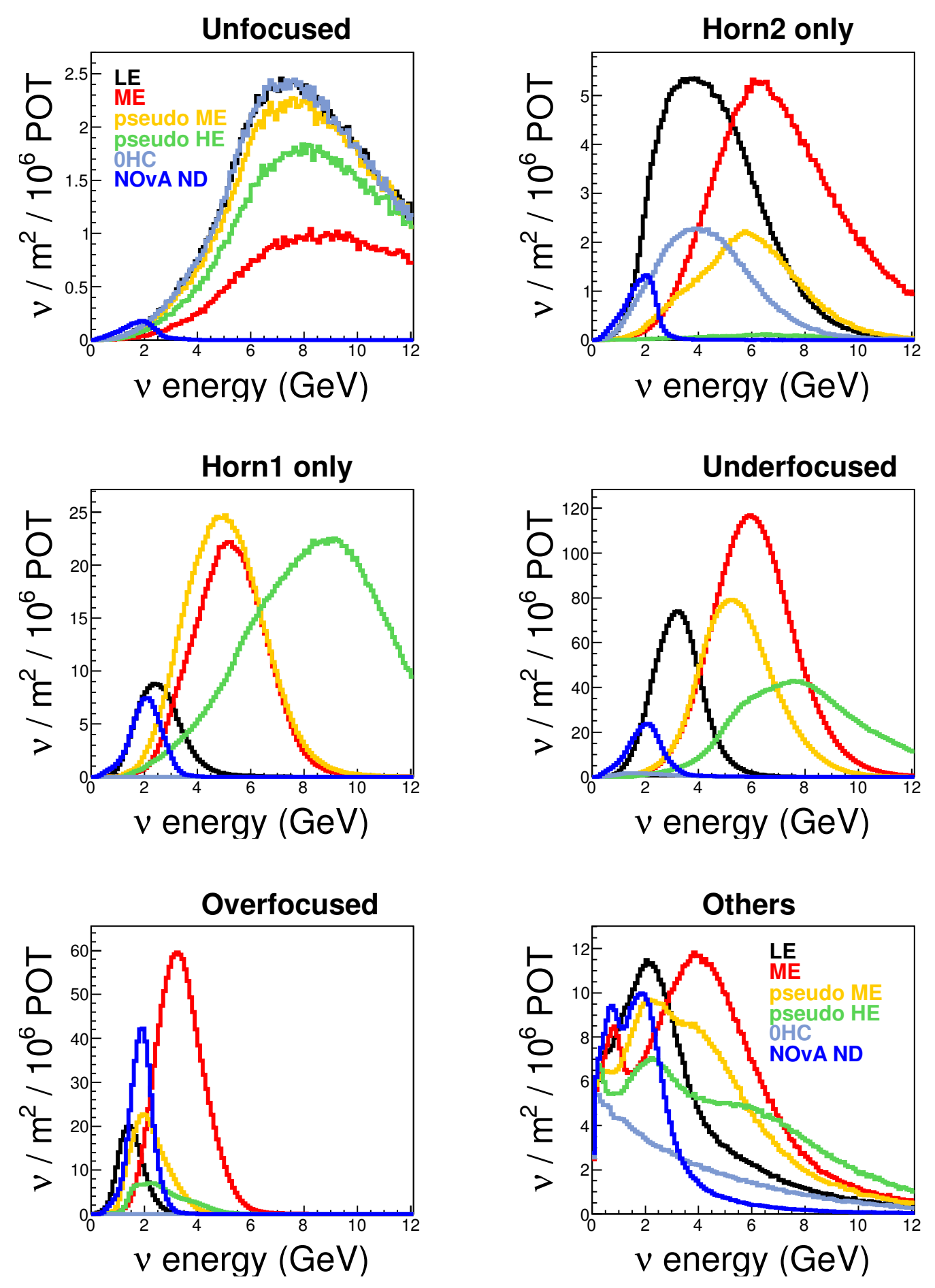

FIG. A.1: Longitudinal vs. transverse momentum yield per $\pi^{+} \nu_{\mu}$ parent. 


\section{Unfocused}
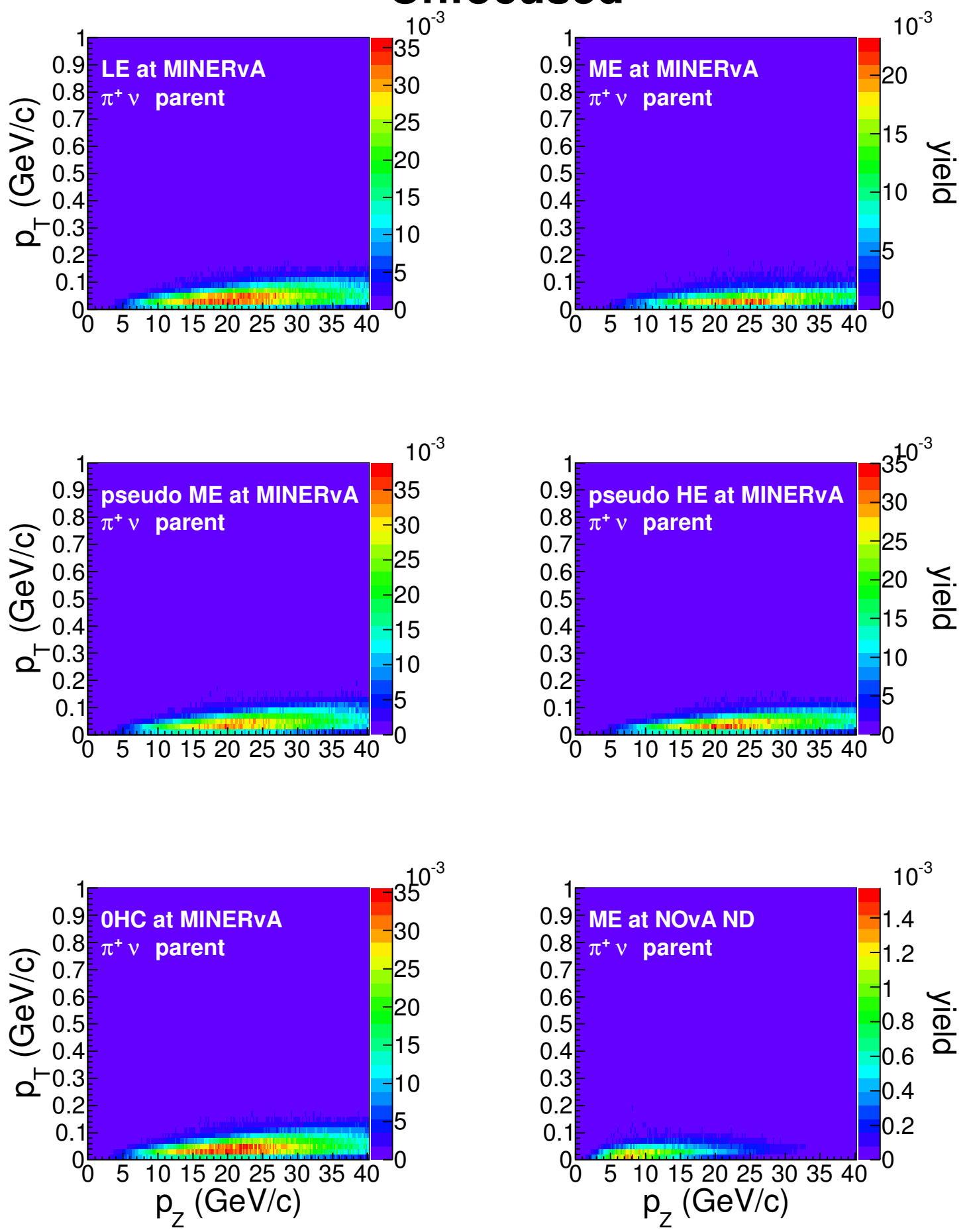

FIG. A.2: Unfocused components of $\pi^{+} \nu_{\mu}$ parent. 


\section{Horn2 only}
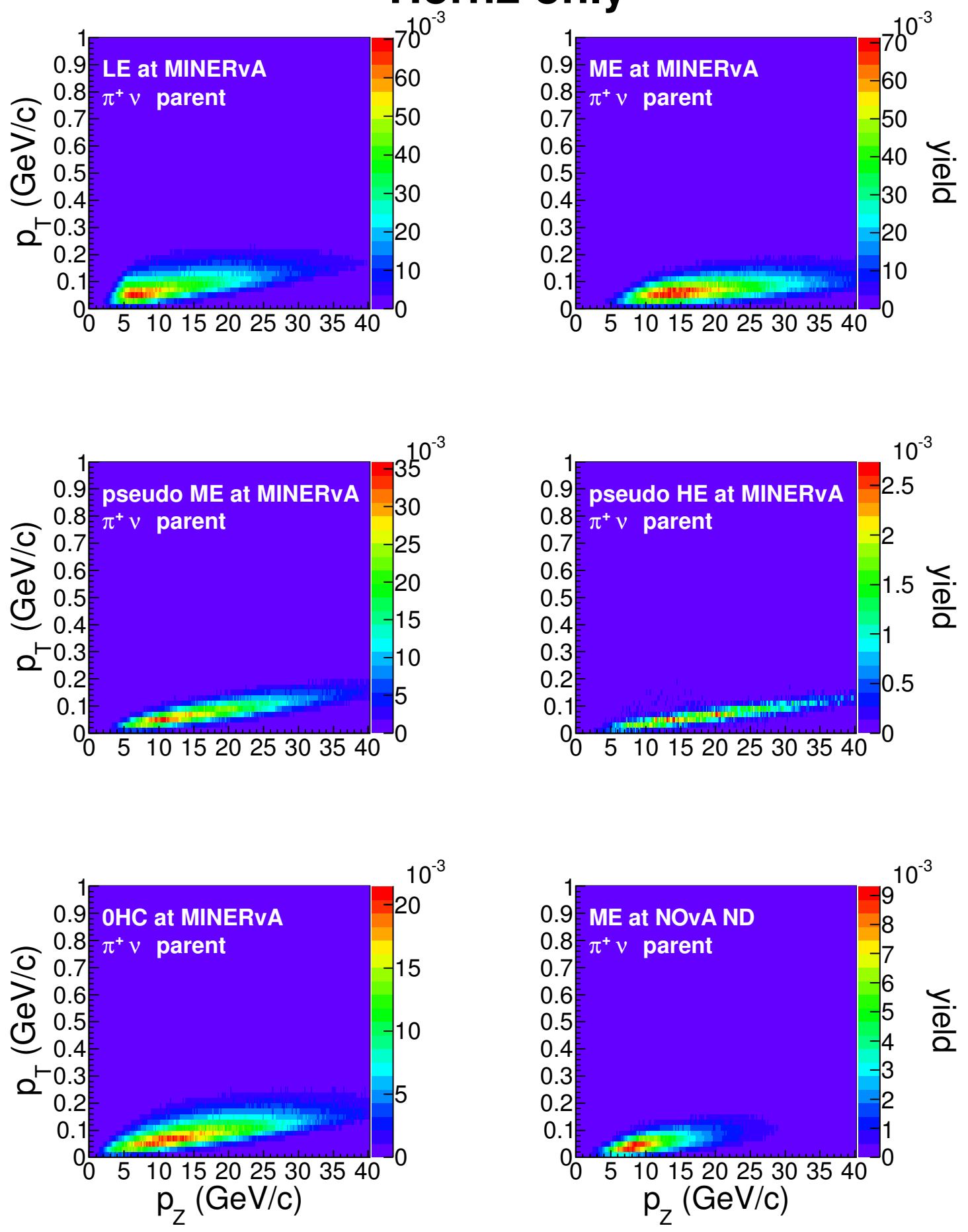

FIG. A.3: H2 only components of $\pi^{+} \nu_{\mu}$ parent. 


\section{Horn1 only}
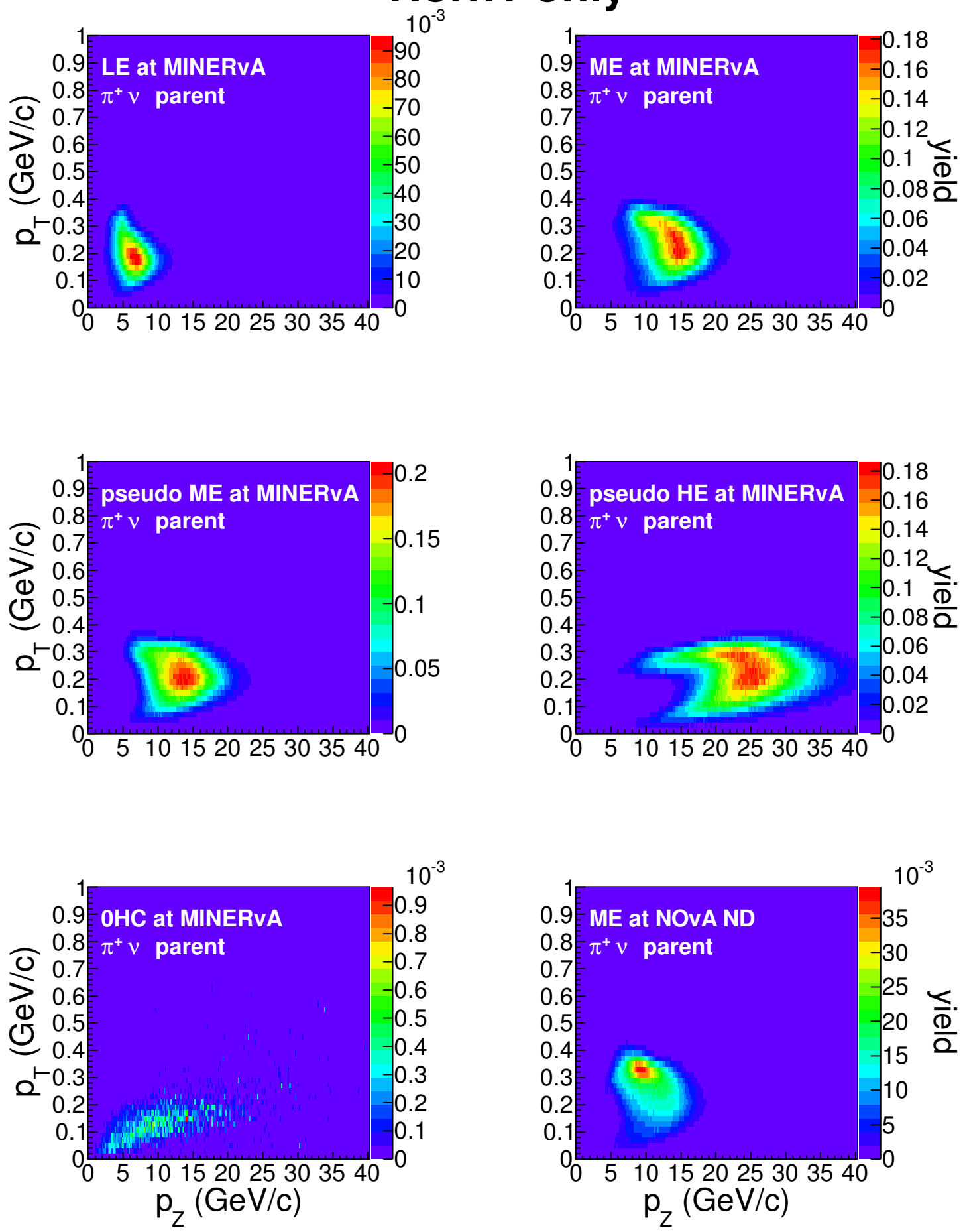

FIG. A.4: Horn1 only components of $\pi^{+} \nu_{\mu}$ parent. 


\section{Underfocused}
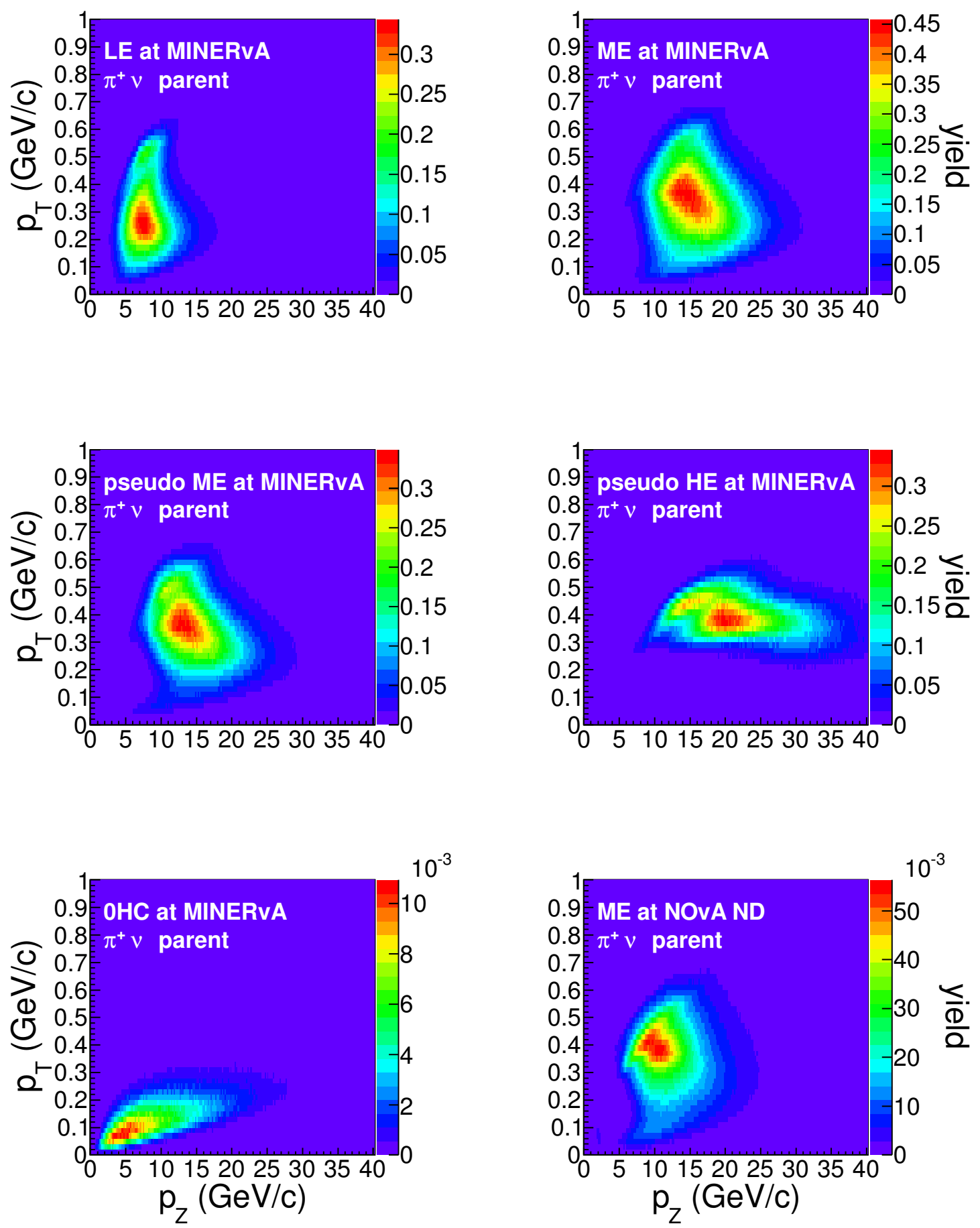

FIG. A.5: Underfocused components of $\pi^{+} \nu_{\mu}$ parent. 


\section{Overfocused}
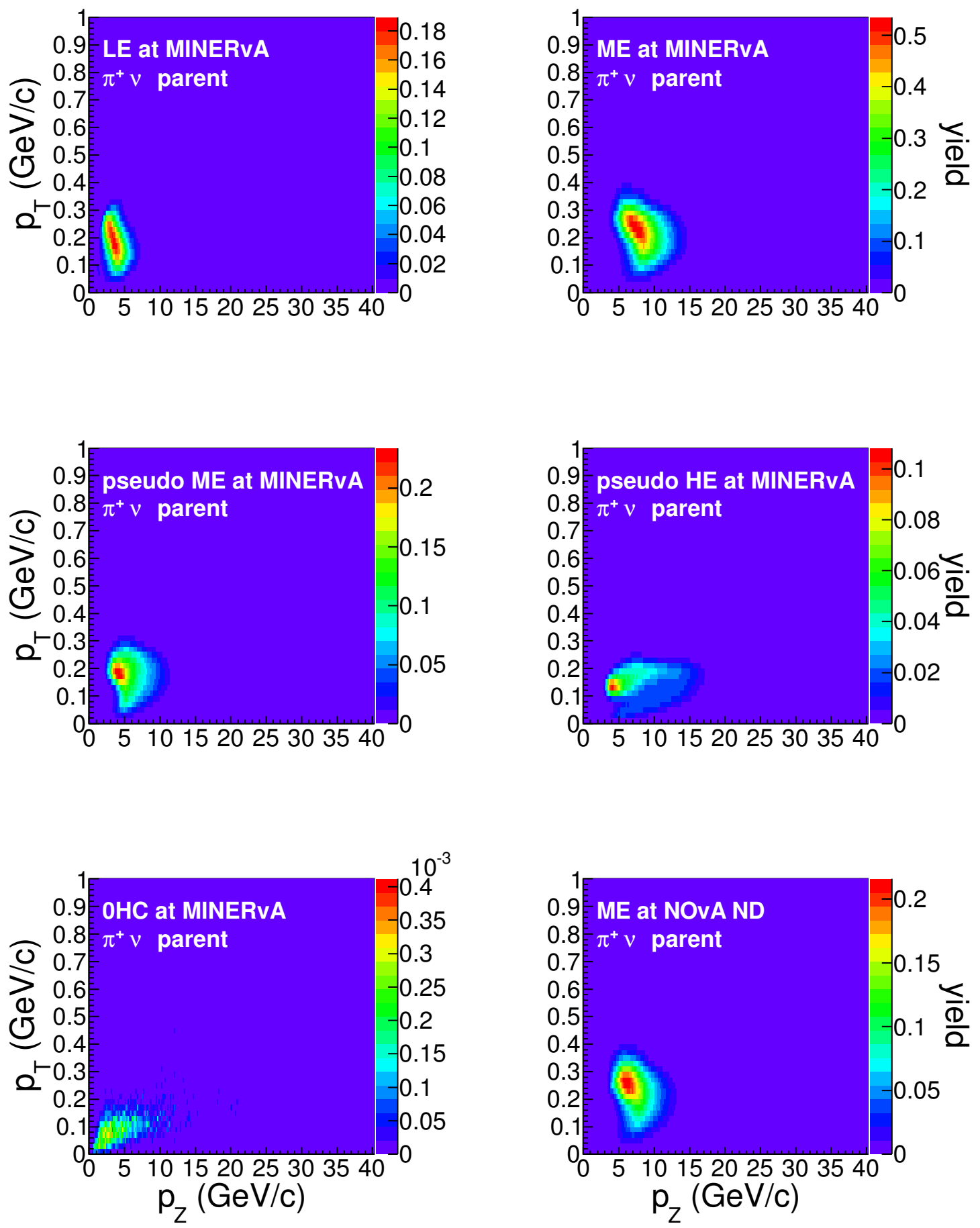

FIG. A.6: Overfocused components of $\pi^{+} \nu_{\mu}$ parent. 

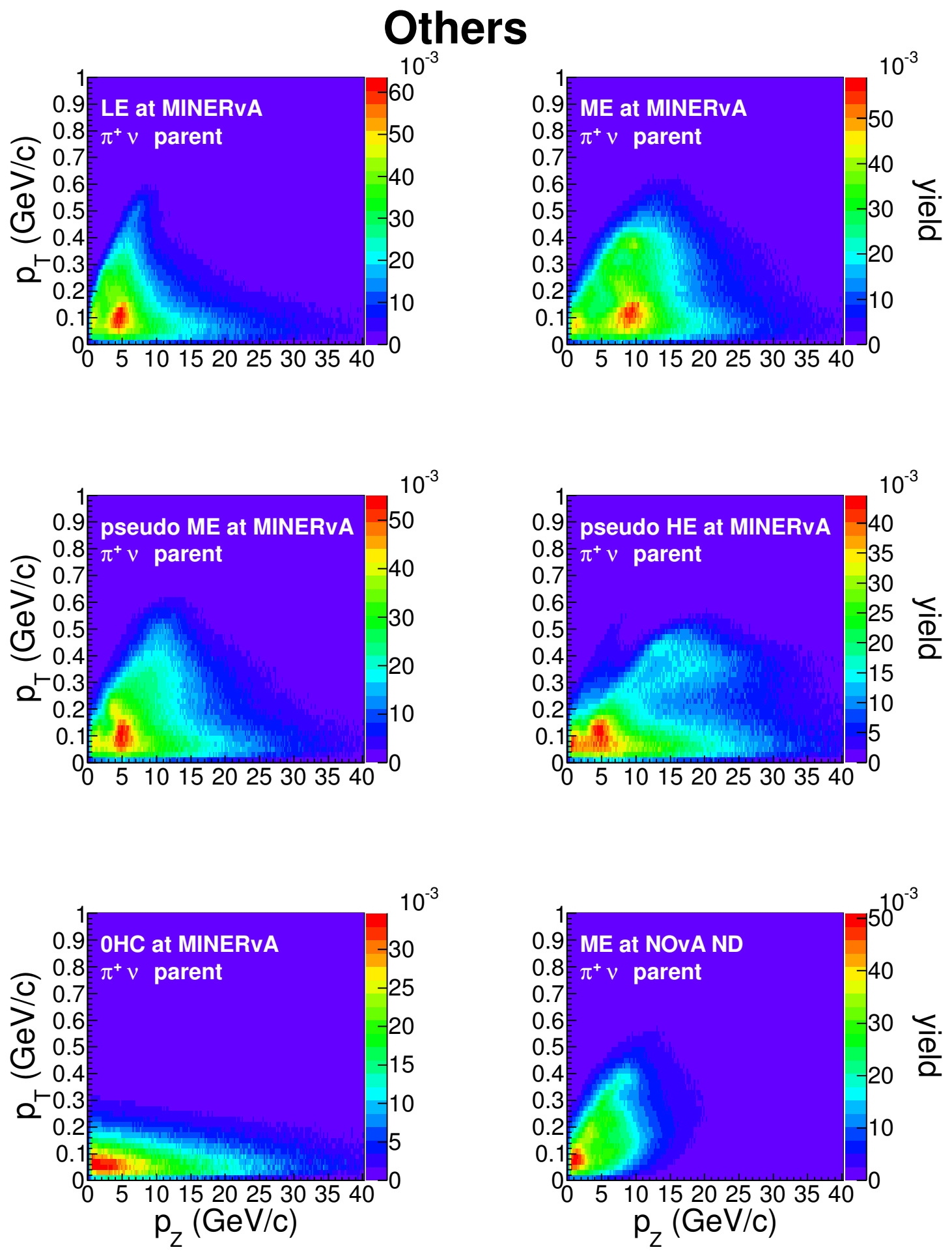

FIG. A.7: Other components of $\pi^{+} \nu_{\mu}$ parent. 


\section{A.2 Flux HP leading to neutrino in NOvA}

This appendix contains the hadronic cascade in NuMI leading to a muon neutrino and electron neutrino in the center of the NOvA front face:

- Focusing components (Figure A.8).

- Neutrino parents (Figure A.9).

- Neutrino grandparents (Figure A.10).

- Interaction map (Figure A.11 and A.12).

- Hadronic production in the target (Figures A.13).

- Beam attenuation (Figure A.14). 


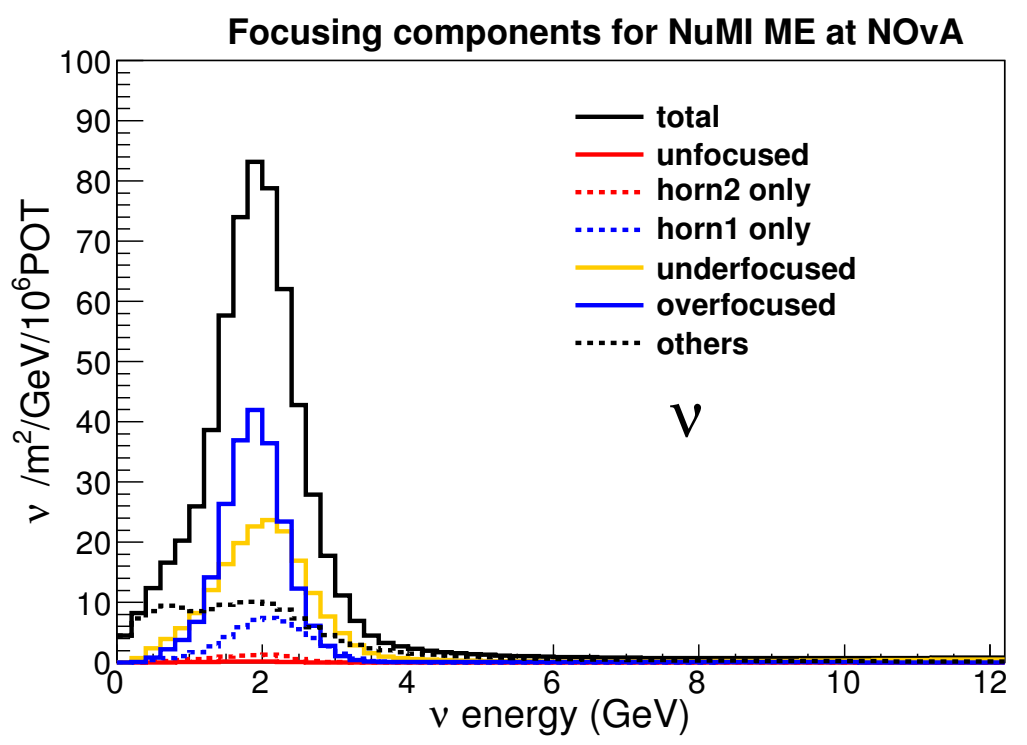

(a) $\nu_{\mu}$ components

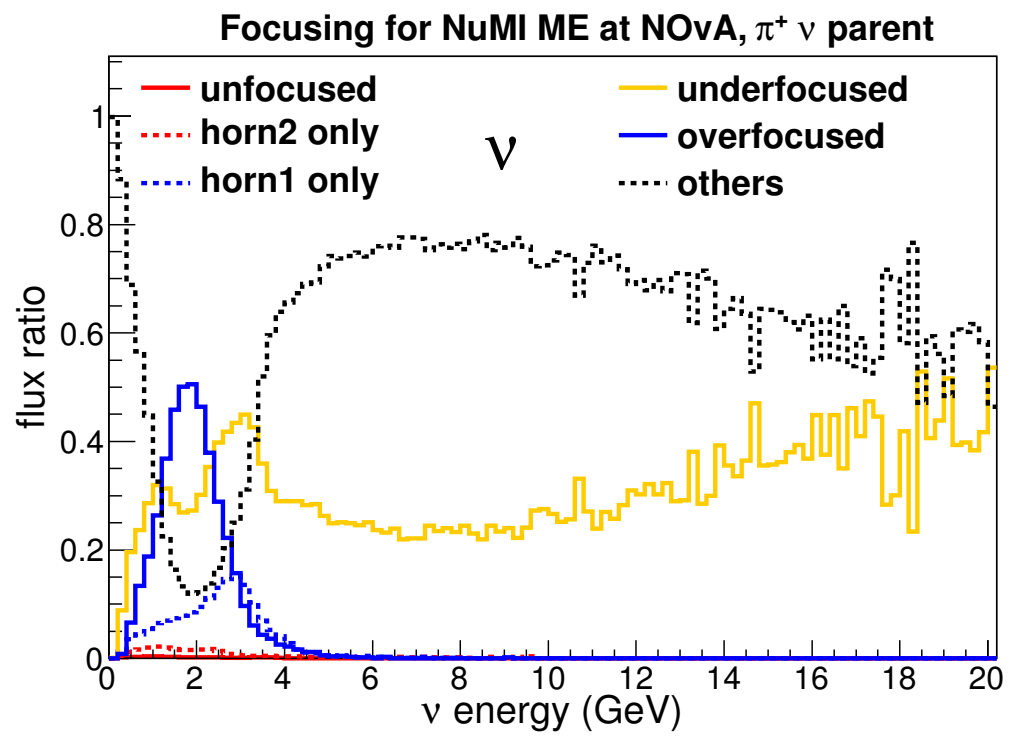

(b) $\pi^{+} \nu_{\mu}$ parents

FIG. A.8: Focusing components for NOvA ND. 

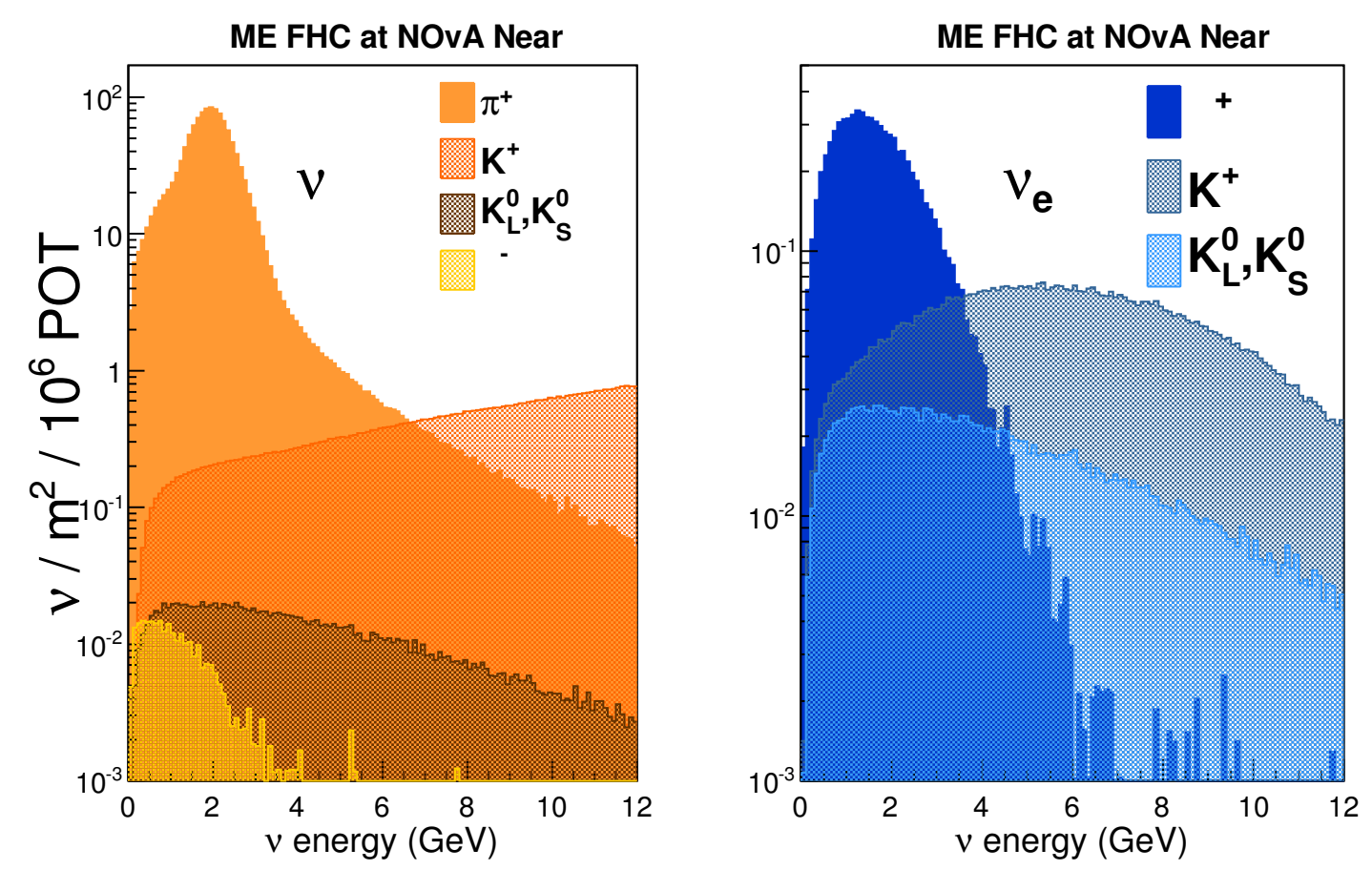

FIG. A.9: Muon and electron neutrino parents in NOvA. 

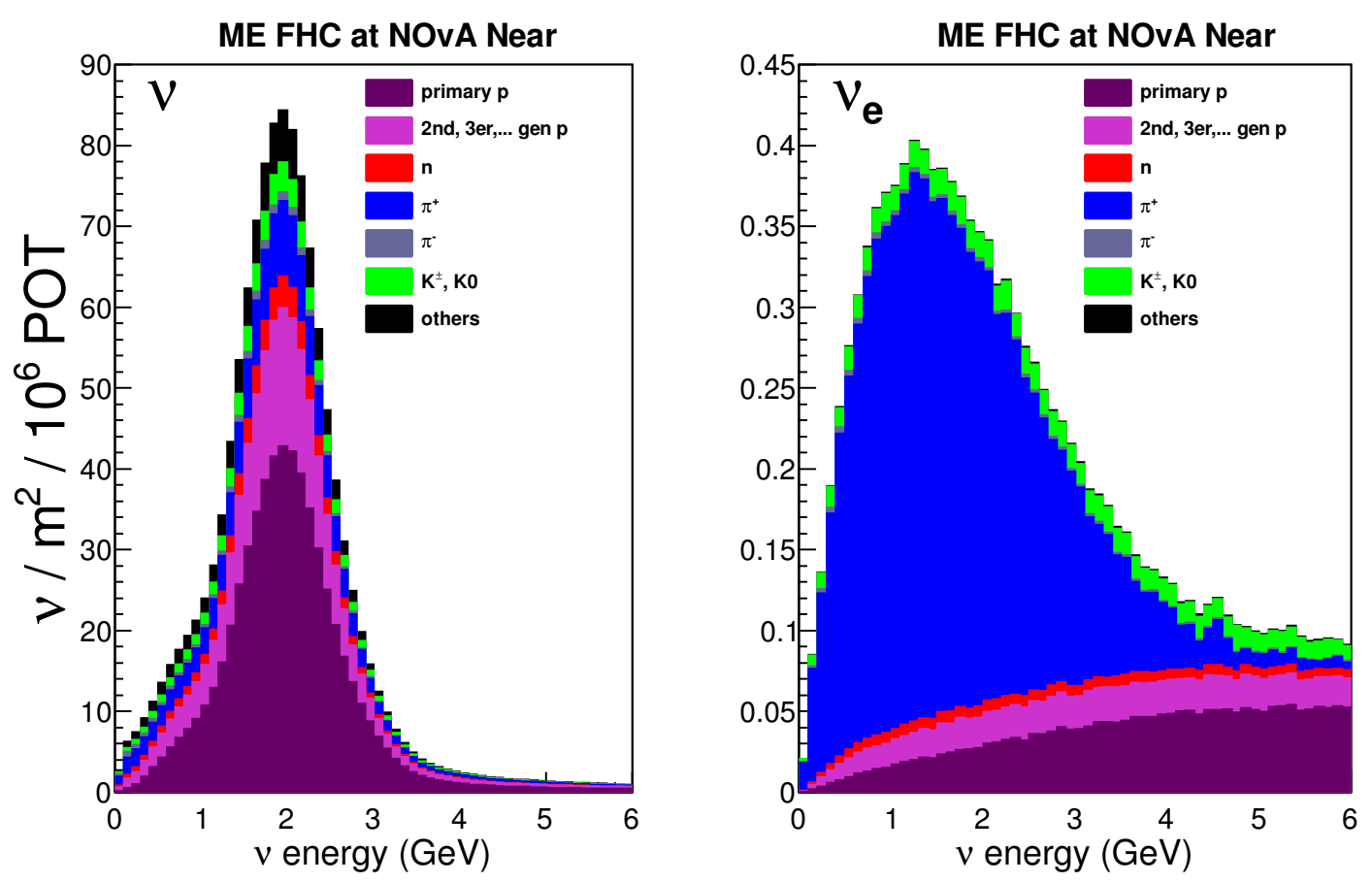

FIG. A.10: Muon and electron neutrino grandparents in NOvA. 


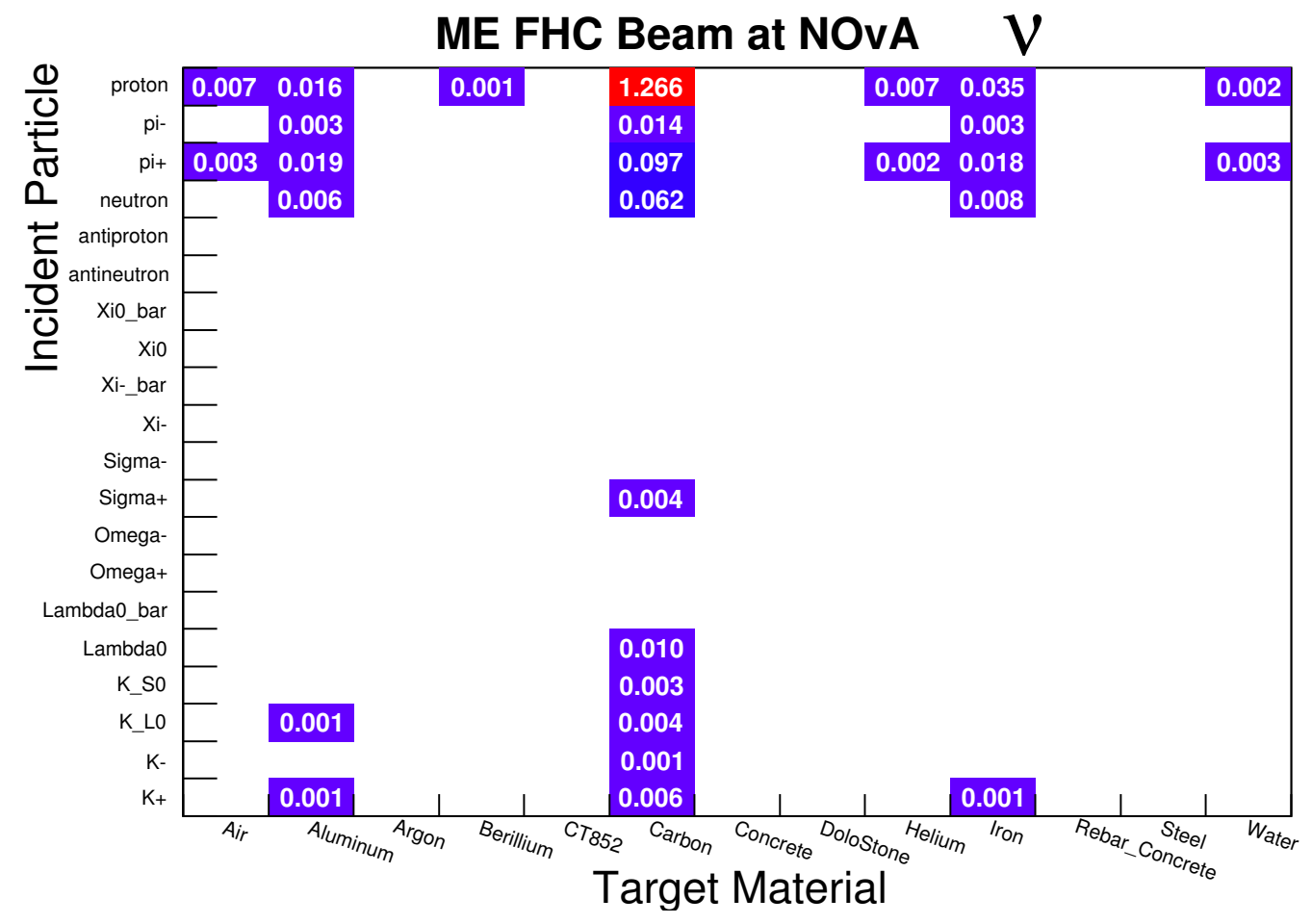

FIG. A.11: Interaction map for $\nu_{\mu}$ in NOvA. 


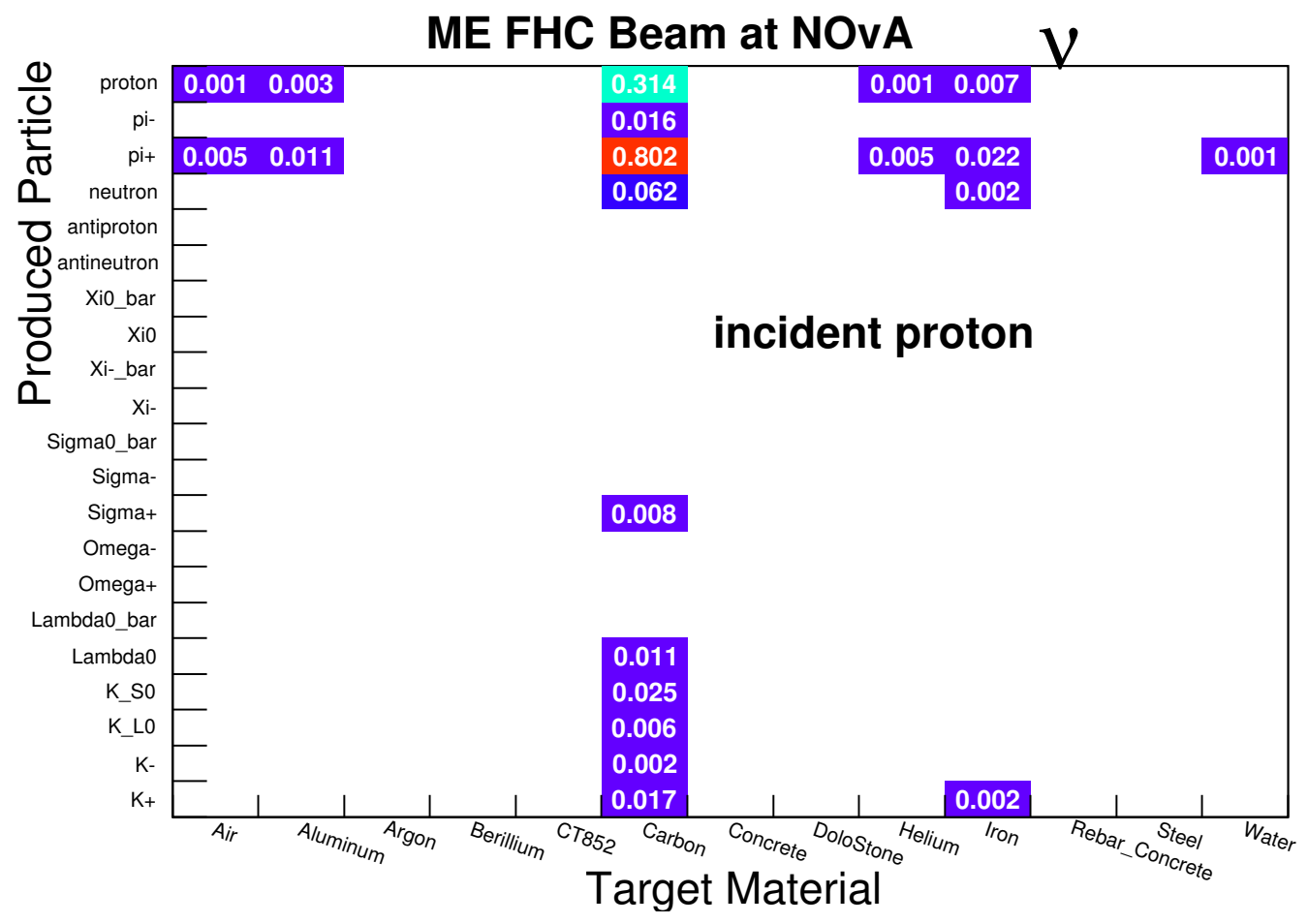

FIG. A.12: Incident protons for $\nu_{\mu}$ in NOvA. 

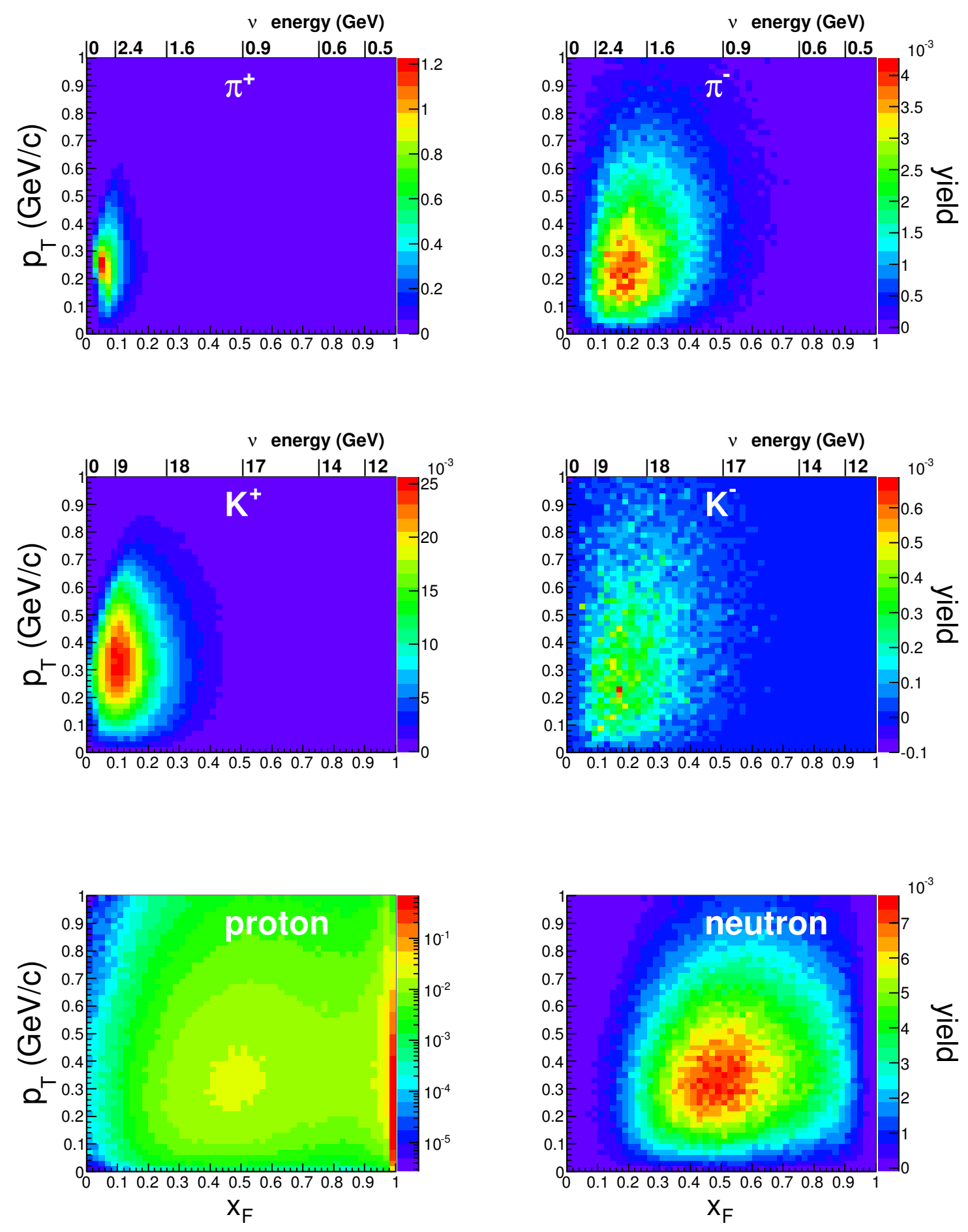

FIG. A.13: Hadron production leading to $\nu_{\mu}$ in NOvA. 

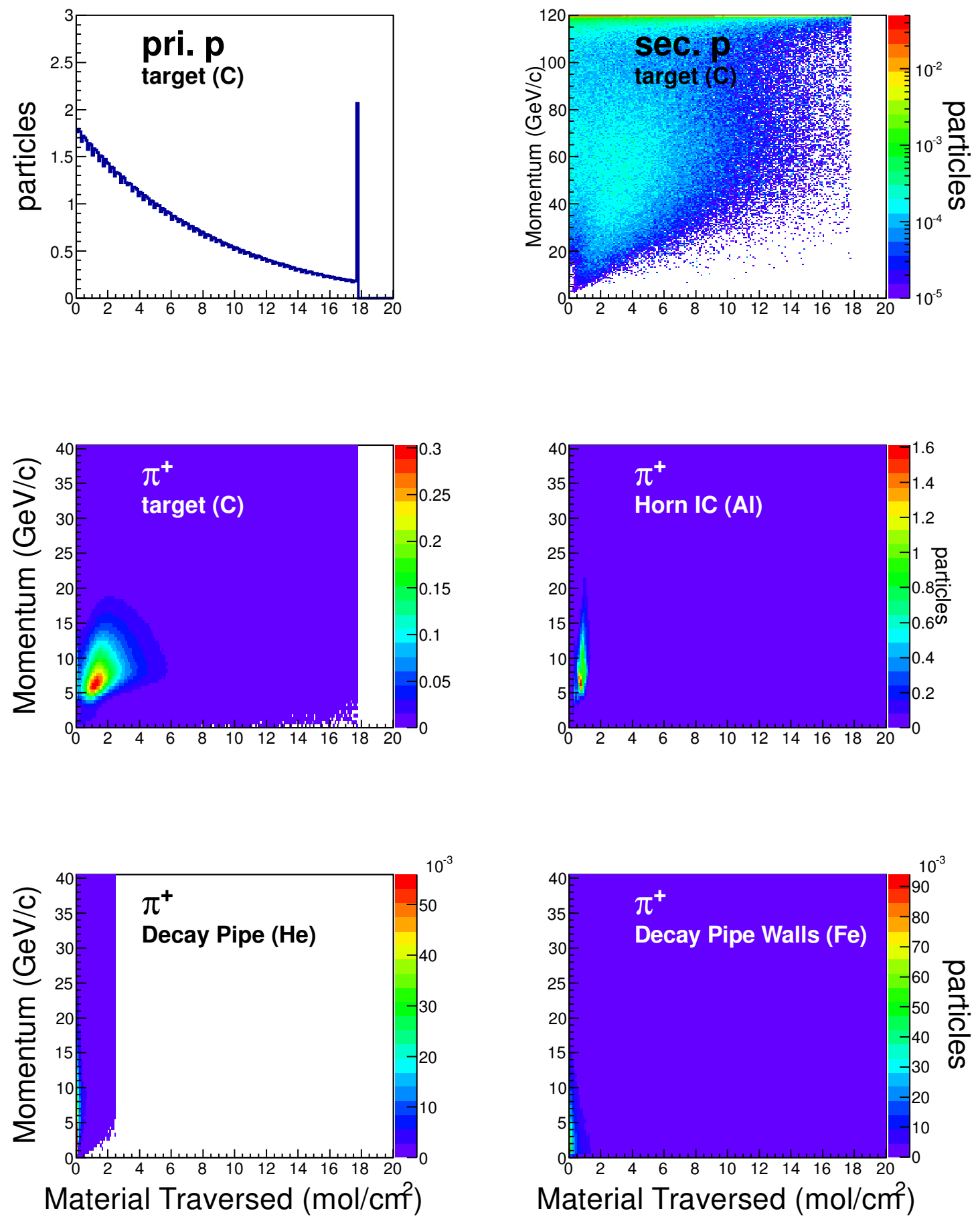

FIG. A.14: Beam attenuation for particles leading to $\nu_{\mu}$ in NOvA. 


\section{APPENDIX B}

\section{NuMI flux tables}

This appendix contains tables of the Generation 2 flux results:

- MINERvA Gen2-thin Low Energy flux for $\nu_{\mu}, \bar{\nu}_{\mu}$ and $\nu_{e}$ at MINERvA (table B.1).

- MINERvA Gen2-thick Low Energy flux for $\nu_{\mu}, \bar{\nu}_{\mu}$ and $\nu_{e}$ at MINERvA (table B.2).

- MINERvA Gen2-thin Medium Energy flux for $\nu_{\mu}$ and $\nu_{e}$ at MINERvA (table B.3).

- NOvA Gen2-thin Low Energy flux for $\nu_{\mu}$ at NOvA Near Detector (table B.4). 


\begin{tabular}{|c|c|c|c|c|c|c|c|c|c|}
\hline$\Delta E(G e V)$ & $\phi_{\nu_{\mu}}$ & $\delta \phi_{\nu_{\mu}}^{H P}$ & $\delta \phi_{\nu_{\mu}}^{F o c}$ & $\phi_{\bar{\nu}_{\mu}}$ & $\delta \phi_{\bar{\nu}_{\mu}}^{H P}$ & $\delta \phi_{\bar{\nu}_{\mu}}^{F o c}$ & $\phi_{\nu_{e}}$ & $\delta \phi_{\nu_{e}}^{H P}$ & $\delta \phi_{\nu_{e}}^{F o c}$ \\
\hline$[0,0.5]$ & 2.82 & 15.7 & 2.3 & 2.17 & 10.4 & 2.3 & 0.08 & 16.6 & 2.3 \\
\hline$[0.5,1]$ & 6.75 & 10.6 & 3.1 & 6.35 & 9.9 & 3.1 & 0.19 & 9.1 & 3.1 \\
\hline$[1,1.5]$ & 17.36 & 8.1 & 2.2 & 16.35 & 7.3 & 2.2 & 0.34 & 6.5 & 2.2 \\
\hline$[1.5,2]$ & 25.86 & 7.6 & 2.6 & 23.53 & 7.0 & 2.6 & 0.41 & 6.8 & 2.6 \\
\hline$[2,2.5]$ & 34.00 & 7.6 & 2.8 & 30.32 & 7.1 & 2.8 & 0.44 & 6.5 & 2.8 \\
\hline$[2.5,3]$ & 41.73 & 7.2 & 3.1 & 36.84 & 6.5 & 3.1 & 0.39 & 6.3 & 3.1 \\
\hline$[3,3.5]$ & 43.79 & 6.5 & 3.5 & 37.39 & 5.7 & 3.5 & 0.36 & 6.1 & 3.5 \\
\hline$[3.5,4]$ & 35.09 & 6.1 & 5.4 & 29.00 & 5.1 & 5.4 & 0.32 & 5.9 & 5.4 \\
\hline$[4,4.5]$ & 21.18 & 6.1 & 9.1 & 16.81 & 5.0 & 9.1 & 0.26 & 6.1 & 9.1 \\
\hline$[4.5,5]$ & 11.54 & 6.4 & 10.4 & 8.75 & 5.4 & 10.4 & 0.23 & 6.3 & 10.4 \\
\hline$[5,5.5]$ & 7.05 & 7.0 & 7.6 & 5.15 & 6.2 & 7.6 & 0.18 & 5.8 & 7.6 \\
\hline$[5.5,6]$ & 5.02 & 8.1 & 4.5 & 3.46 & 7.0 & 4.5 & 0.15 & 5.1 & 4.5 \\
\hline$[6,6.5]$ & 4.08 & 8.5 & 3.1 & 2.62 & 7.5 & 3.1 & 0.11 & 5.5 & 3.1 \\
\hline$[6.5,7]$ & 3.41 & 8.8 & 2.7 & 2.14 & 7.8 & 2.7 & 0.09 & 5.6 & 2.7 \\
\hline$[7,7.5]$ & 2.97 & 9.0 & 2.5 & 1.77 & 7.9 & 2.5 & 0.06 & 5.9 & 2.5 \\
\hline$[7.5,8]$ & 2.62 & 9.0 & 2.5 & 1.48 & 8.2 & 2.5 & 0.06 & 5.6 & 2.5 \\
\hline$[8,8.5]$ & 2.34 & 9.1 & 2.4 & 1.24 & 7.9 & 2.4 & 0.04 & 6.1 & 2.4 \\
\hline$[8.5,9]$ & 2.11 & 9.1 & 2.3 & 1.09 & 8.3 & 2.3 & 0.04 & 6.5 & 2.3 \\
\hline$[9,9.5]$ & 1.91 & 9.1 & 2.2 & 0.99 & 7.9 & 2.2 & 0.04 & 7.7 & 2.2 \\
\hline$[9.5,10]$ & 1.74 & 8.9 & 2.2 & 0.88 & 8.2 & 2.2 & 0.04 & 8.4 & 2.2 \\
\hline$[10,10.5]$ & 1.58 & 9.0 & 2.1 & 0.74 & 8.2 & 2.1 & 0.03 & 8.5 & 2.1 \\
\hline$[10.5,11]$ & 1.47 & 8.8 & 2.1 & 0.71 & 8.3 & 2.1 & 0.03 & 8.0 & 2.1 \\
\hline$[11,11.5]$ & 1.33 & 8.7 & 2.0 & 0.62 & 8.2 & 2.0 & 0.03 & 7.8 & 2.0 \\
\hline$[11.5,12]$ & 1.21 & 8.6 & 2.0 & 0.58 & 8.0 & 2.0 & 0.02 & 8.1 & 2.0 \\
\hline$[12,12.5]$ & 1.10 & 8.6 & 2.0 & 0.50 & 8.2 & 2.0 & 0.02 & 8.3 & 2.0 \\
\hline$[12.5,13]$ & 1.01 & 8.6 & 2.0 & 0.47 & 8.0 & 2.0 & 0.02 & 10.8 & 2.0 \\
\hline$[13,13.5]$ & 0.91 & 8.5 & 2.0 & 0.42 & 8.1 & 2.0 & 0.02 & 9.0 & 2.0 \\
\hline$[13.5,14]$ & 0.86 & 8.5 & 2.0 & 0.37 & 8.2 & 2.0 & 0.02 & 9.5 & 2.0 \\
\hline$[14,14.5]$ & 0.78 & 8.5 & 2.0 & 0.34 & 7.9 & 2.0 & 0.02 & 10.7 & 2.0 \\
\hline$[14.5,15]$ & 0.71 & 8.3 & 2.0 & 0.31 & 8.4 & 2.0 & 0.02 & 9.7 & 2.0 \\
\hline$[15,15.5]$ & 0.64 & 8.4 & 2.0 & 0.27 & 8.6 & 2.0 & 0.01 & 11.0 & 2.0 \\
\hline$[15.5,16]$ & 0.59 & 8.4 & 2.0 & 0.26 & 8.6 & 2.0 & 0.01 & 11.7 & 2.0 \\
\hline$[16,16.5]$ & 0.55 & 8.4 & 2.0 & 0.23 & 8.9 & 2.0 & 0.02 & 10.9 & 2.0 \\
\hline$[16.5,17]$ & 0.49 & 8.5 & 2.0 & 0.19 & 9.2 & 2.0 & 0.01 & 10.5 & 2.0 \\
\hline$[17,17.5]$ & 0.45 & 8.3 & 2.0 & 0.19 & 8.8 & 2.0 & 0.01 & 10.8 & 2.0 \\
\hline$[17.5,18]$ & 0.41 & 8.6 & 2.0 & 0.17 & 8.8 & 2.0 & 0.01 & 10.9 & 2.0 \\
\hline$[18,18.5]$ & 0.37 & 8.4 & 2.0 & 0.15 & 8.9 & 2.0 & 0.01 & 11.6 & 2.0 \\
\hline$[18.5,19]$ & 0.34 & 8.7 & 2.0 & 0.13 & 10.2 & 2.0 & 0.01 & 11.7 & 2.0 \\
\hline$[19,19.5]$ & 0.32 & 8.8 & 2.0 & 0.12 & 11.1 & 2.0 & 0.01 & 11.2 & 2.0 \\
\hline$[19.5,20]$ & 0.29 & 9.3 & 2.0 & 0.12 & 10.9 & 2.0 & 0.01 & 10.9 & 2.0 \\
\hline
\end{tabular}

TABLE B.1: Generation2 - thin Low Energy flux results at MINERvA. Flux units: $\nu / m^{2} / 10^{6} P O T$. Uncertainties are given in percentage. 


\begin{tabular}{|c|c|c|c|c|c|c|c|c|c|}
\hline$\Delta E(G e V)$ & $\phi_{\nu_{\mu}}$ & $\delta \phi_{\nu_{\mu}}^{H P}$ & $\delta \phi_{\nu_{\mu}}^{F o c}$ & $\phi_{\bar{\nu}_{\mu}}$ & $\delta \phi_{\bar{\nu}_{\mu}}^{H P}$ & $\delta \phi_{\bar{\nu}_{\mu}}^{F o c}$ & $\phi_{\nu_{e}}$ & $\delta \phi_{\nu_{e}}^{H P}$ & $\delta \phi_{\nu_{e}}^{F o c}$ \\
\hline$[0,0.5]$ & 3.54 & 10.0 & 2.3 & 2.88 & 7.3 & 2.3 & 0.08 & 14.7 & 2.3 \\
\hline$[0.5,1]$ & 7.35 & 7.8 & 3.1 & 6.86 & 7.4 & 3.1 & 0.19 & 5.3 & 3.1 \\
\hline$[1,1.5]$ & 17.29 & 7.4 & 2.2 & 16.31 & 6.7 & 2.2 & 0.33 & 4.1 & 2.2 \\
\hline$[1.5,2]$ & 25.36 & 4.9 & 2.6 & 23.74 & 4.7 & 2.6 & 0.41 & 4.3 & 2.6 \\
\hline$[2,2.5]$ & 33.72 & 5.0 & 2.8 & 30.96 & 5.0 & 2.8 & 0.44 & 3.9 & 2.8 \\
\hline$[2.5,3]$ & 42.91 & 4.6 & 3.1 & 39.03 & 5.0 & 3.1 & 0.38 & 3.9 & 3.1 \\
\hline$[3,3.5]$ & 44.38 & 4.6 & 3.5 & 38.46 & 4.9 & 3.5 & 0.36 & 3.8 & 3.5 \\
\hline$[3.5,4]$ & 34.54 & 4.9 & 5.4 & 29.25 & 5.0 & 5.4 & 0.32 & 3.9 & 5.4 \\
\hline$[4,4.5]$ & 19.75 & 4.7 & 9.1 & 16.92 & 4.8 & 9.1 & 0.26 & 3.8 & 9.1 \\
\hline$[4.5,5]$ & 10.36 & 4.6 & 10.4 & 8.69 & 4.9 & 10.4 & 0.23 & 3.8 & 10.4 \\
\hline$[5,5.5]$ & 6.16 & 4.7 & 7.6 & 5.02 & 5.2 & 7.6 & 0.17 & 3.7 & 7.6 \\
\hline$[5.5,6]$ & 4.32 & 4.8 & 4.5 & 3.36 & 5.4 & 4.5 & 0.14 & 3.4 & 4.5 \\
\hline$[6,6.5]$ & 3.46 & 4.9 & 3.1 & 2.51 & 5.4 & 3.1 & 0.10 & 3.8 & 3.1 \\
\hline$[6.5,7]$ & 2.85 & 5.0 & 2.7 & 2.01 & 5.6 & 2.7 & 0.08 & 3.8 & 2.7 \\
\hline$[7,7.5]$ & 2.46 & 5.0 & 2.5 & 1.63 & 5.7 & 2.5 & 0.06 & 4.0 & 2.5 \\
\hline$[7.5,8]$ & 2.16 & 5.0 & 2.5 & 1.35 & 5.9 & 2.5 & 0.06 & 3.5 & 2.5 \\
\hline$[8,8.5]$ & 1.95 & 5.1 & 2.4 & 1.12 & 6.0 & 2.4 & 0.04 & 3.9 & 2.4 \\
\hline$[8.5,9]$ & 1.77 & 5.2 & 2.3 & 0.99 & 6.1 & 2.3 & 0.04 & 3.8 & 2.3 \\
\hline$[9,9.5]$ & 1.61 & 5.2 & 2.2 & 0.91 & 6.1 & 2.2 & 0.03 & 4.7 & 2.2 \\
\hline$[9.5,10]$ & 1.47 & 5.2 & 2.2 & 0.82 & 6.2 & 2.2 & 0.03 & 4.7 & 2.2 \\
\hline$[10,10.5]$ & 1.34 & 5.3 & 2.1 & 0.70 & 6.4 & 2.1 & 0.03 & 5.4 & 2.1 \\
\hline$[10.5,11]$ & 1.25 & 5.2 & 2.1 & 0.66 & 6.2 & 2.1 & 0.03 & 5.1 & 2.1 \\
\hline$[11,11.5]$ & 1.13 & 5.1 & 2.0 & 0.58 & 6.6 & 2.0 & 0.02 & 4.3 & 2.0 \\
\hline$[11.5,12]$ & 1.04 & 5.1 & 2.0 & 0.54 & 6.9 & 2.0 & 0.02 & 4.1 & 2.0 \\
\hline$[12,12.5]$ & 0.96 & 5.0 & 2.0 & 0.47 & 6.8 & 2.0 & 0.02 & 4.9 & 2.0 \\
\hline$[12.5,13]$ & 0.90 & 5.0 & 2.0 & 0.44 & 6.7 & 2.0 & 0.02 & 6.4 & 2.0 \\
\hline$[13,13.5]$ & 0.83 & 4.8 & 2.0 & 0.40 & 6.5 & 2.0 & 0.02 & 4.7 & 2.0 \\
\hline$[13.5,14]$ & 0.80 & 4.9 & 2.0 & 0.36 & 6.5 & 2.0 & 0.02 & 5.5 & 2.0 \\
\hline$[14,14.5]$ & 0.75 & 4.9 & 2.0 & 0.33 & 6.7 & 2.0 & 0.01 & 4.4 & 2.0 \\
\hline$[14.5,15]$ & 0.69 & 4.9 & 2.0 & 0.30 & 7.0 & 2.0 & 0.01 & 4.1 & 2.0 \\
\hline$[15,15.5]$ & 0.63 & 4.8 & 2.0 & 0.26 & 7.0 & 2.0 & 0.01 & 5.2 & 2.0 \\
\hline$[15.5,16]$ & 0.59 & 4.7 & 2.0 & 0.24 & 7.3 & 2.0 & 0.01 & 5.4 & 2.0 \\
\hline$[16,16.5]$ & 0.55 & 4.7 & 2.0 & 0.21 & 7.6 & 2.0 & 0.01 & 4.7 & 2.0 \\
\hline$[16.5,17]$ & 0.50 & 4.7 & 2.0 & 0.18 & 8.0 & 2.0 & 0.01 & 5.3 & 2.0 \\
\hline$[17,17.5]$ & 0.46 & 4.7 & 2.0 & 0.17 & 8.2 & 2.0 & 0.01 & 4.9 & 2.0 \\
\hline$[17.5,18]$ & 0.42 & 4.7 & 2.0 & 0.15 & 7.8 & 2.0 & 0.01 & 4.7 & 2.0 \\
\hline$[18,18.5]$ & 0.38 & 4.9 & 2.0 & 0.13 & 7.7 & 2.0 & 0.01 & 5.0 & 2.0 \\
\hline$[18.5,19]$ & 0.34 & 5.0 & 2.0 & 0.12 & 8.4 & 2.0 & 0.01 & 5.7 & 2.0 \\
\hline$[19,19.5]$ & 0.32 & 5.2 & 2.0 & 0.11 & 8.8 & 2.0 & 0.01 & 5.7 & 2.0 \\
\hline$[19.5,20]$ & 0.30 & 5.6 & 2.0 & 0.10 & 9.0 & 2.0 & 0.01 & 5.1 & 2.0 \\
\hline
\end{tabular}

TABLE B.2: Generation2 - thick Low Energy flux results at MINERvA. Flux units: $\nu / m^{2} / 10^{6} P O T$. Uncertainties are given in percentage. 


\begin{tabular}{|c|c|c|c|c|c|c|c|c|c|}
\hline$\Delta E$ & $\phi_{\nu_{\mu}}$ & $\delta \phi_{\nu_{\mu}}^{H P}$ & $\delta \phi_{\nu_{\mu}}^{F o c}$ & $\phi_{\bar{\nu}_{\mu}}$ & $\delta \phi_{\bar{\nu}_{\mu}}^{H P}$ & $\phi_{\nu_{e}}$ & $\delta \phi_{\nu_{e}}^{H P}$ & $\phi_{\bar{\nu}_{e}}$ & $\delta \phi_{\bar{\nu}_{e}}^{H P}$ \\
\hline$[0,0.5]$ & 2.45 & 16.5 & 12.8 & 1.64 & 15.5 & 0.04 & 17.3 & 0.00 & 79.4 \\
\hline$[0.5,1]$ & 4.30 & 12.6 & 8.4 & 1.61 & 16.2 & 0.13 & 8.7 & 0.01 & 19.1 \\
\hline$[1,1.5]$ & 4.07 & 11.9 & 5.2 & 1.38 & 15.3 & 0.20 & 8.2 & 0.04 & 18.9 \\
\hline$[1.5,2]$ & 8.39 & 9.2 & 3.6 & 1.32 & 15.6 & 0.29 & 7.5 & 0.02 & 17.4 \\
\hline$[2,2.5]$ & 19.96 & 8.1 & 3.0 & 1.22 & 14.0 & 0.36 & 7.0 & 0.02 & 16.0 \\
\hline$[2.5,3]$ & 33.83 & 7.5 & 2.3 & 1.27 & 13.1 & 0.41 & 7.2 & 0.02 & 14.0 \\
\hline$[3,3.5]$ & 44.07 & 7.3 & 3.1 & 1.17 & 12.1 & 0.44 & 6.6 & 0.03 & 13.5 \\
\hline$[3.5,4]$ & 48.68 & 7.4 & 3.8 & 1.24 & 11.5 & 0.45 & 6.9 & 0.02 & 17.5 \\
\hline$[4,4.5]$ & 53.37 & 7.7 & 3.4 & 1.23 & 10.9 & 0.44 & 6.8 & 0.01 & 13.9 \\
\hline$[4.5,5]$ & 60.55 & 7.9 & 3.2 & 1.16 & 10.8 & 0.42 & 7.1 & 0.01 & 11.2 \\
\hline$[5,5.5]$ & 66.98 & 7.8 & 3.2 & 1.15 & 10.4 & 0.40 & 7.1 & 0.01 & 14.6 \\
\hline$[5.5,6]$ & 69.57 & 7.5 & 3.5 & 1.08 & 9.9 & 0.38 & 6.8 & 0.02 & 13.1 \\
\hline$[6,6.5]$ & 66.23 & 7.1 & 4.6 & 1.01 & 9.9 & 0.34 & 6.5 & 0.01 & 9.9 \\
\hline$[6.5,7]$ & 56.85 & 6.8 & 6.7 & 0.96 & 9.1 & 0.31 & 6.5 & 0.01 & 10.5 \\
\hline$[7,7.5]$ & 43.61 & 6.7 & 9.4 & 0.84 & 9.3 & 0.27 & 6.5 & 0.01 & 10.5 \\
\hline$[7.5,8]$ & 30.81 & 6.6 & 12.5 & 0.77 & 9.5 & 0.23 & 7.3 & 0.01 & 13.0 \\
\hline$[8,8.5]$ & 20.79 & 6.7 & 15.7 & 0.65 & 8.7 & 0.20 & 6.7 & 0.01 & 12.7 \\
\hline$[8.5,9]$ & 13.53 & 6.8 & 18.4 & 0.58 & 8.6 & 0.18 & 6.3 & 0.01 & 9.8 \\
\hline$[9,9.5]$ & 8.75 & 7.0 & 19.4 & 0.54 & 8.7 & 0.15 & 6.6 & 0.01 & 13.7 \\
\hline$[9.5,10]$ & 5.76 & 7.3 & 18.1 & 0.49 & 8.8 & 0.14 & 6.3 & 0.01 & 11.4 \\
\hline$[10,10.5]$ & 4.03 & 7.6 & 15.9 & 0.46 & 8.7 & 0.12 & 6.4 & 0.01 & 12.0 \\
\hline$[10.5,11]$ & 2.96 & 8.1 & 13.4 & 0.42 & 9.1 & 0.10 & 7.0 & 0.01 & 11.5 \\
\hline$[11,11.5]$ & 2.30 & 8.4 & 11.6 & 0.38 & 9.0 & 0.10 & 7.0 & 0.01 & 11.8 \\
\hline$[11.5,12]$ & 1.92 & 8.5 & 8.0 & 0.36 & 8.9 & 0.08 & 6.3 & 0.00 & 16.7 \\
\hline$[12,12.5]$ & 1.64 & 8.7 & 5.7 & 0.33 & 9.7 & 0.07 & 6.4 & 0.01 & 10.9 \\
\hline$[12.5,13]$ & 1.40 & 8.7 & 6.0 & 0.29 & 9.3 & 0.06 & 6.9 & 0.01 & 16.1 \\
\hline$[13,13.5]$ & 1.29 & 8.6 & 3.1 & 0.27 & 9.4 & 0.06 & 7.0 & 0.01 & 13.1 \\
\hline$[13.5,14]$ & 1.16 & 8.4 & 2.3 & 0.25 & 9.8 & 0.05 & 7.0 & 0.01 & 9.5 \\
\hline$[14,14.5]$ & 1.03 & 8.4 & 2.1 & 0.22 & 10.1 & 0.04 & 6.9 & 0.00 & 12.4 \\
\hline$[14.5,15]$ & 0.93 & 8.1 & 3.0 & 0.19 & 9.5 & 0.04 & 7.4 & 0.00 & 14.9 \\
\hline$[15,15.5]$ & 0.86 & 8.3 & 4.4 & 0.19 & 10.3 & 0.03 & 7.0 & 0.01 & 12.4 \\
\hline$[15.5,16]$ & 0.78 & 8.3 & 2.7 & 0.16 & 10.3 & 0.03 & 8.4 & 0.00 & 14.9 \\
\hline$[16,16.5]$ & 0.73 & 7.9 & 2.4 & 0.14 & 10.7 & 0.03 & 8.9 & 0.00 & 10.3 \\
\hline$[16.5,17]$ & 0.67 & 8.2 & 3.8 & 0.13 & 10.9 & 0.03 & 8.6 & 0.01 & 12.9 \\
\hline$[17,17.5]$ & 0.62 & 8.1 & 4.2 & 0.12 & 10.7 & 0.02 & 9.6 & 0.00 & 10.6 \\
\hline$[17.5,18]$ & 0.56 & 8.1 & 5.7 & 0.10 & 12.2 & 0.03 & 9.3 & 0.00 & 13.5 \\
\hline$[18,18.5]$ & 0.54 & 7.6 & 4.9 & 0.09 & 11.1 & 0.02 & 10.6 & 0.00 & 14.4 \\
\hline$[18.5,19]$ & 0.50 & 7.6 & 3.9 & 0.08 & 11.8 & 0.02 & 9.7 & 0.00 & 18.6 \\
\hline$[19,19.5]$ & 0.48 & 7.6 & 4.4 & 0.07 & 13.3 & 0.02 & 9.5 & 0.00 & 12.9 \\
\hline$[19.5,20]$ & 0.45 & 7.5 & 2.9 & 0.07 & 14.2 & 0.01 & 10.2 & 0.00 & 11.1 \\
\hline
\end{tabular}

TABLE B.3: Generation2 - thin Medium Energy flux results at MINERvA. Flux units: $\nu / m^{2} / 10^{6} P O T$. Uncertainties are given in percentage. 


\begin{tabular}{|l|cc||l|cc|}
\hline$\Delta E(G e V)$ & $\phi_{\nu_{\mu}}$ & $\delta \phi_{\nu_{\mu}}^{H P}$ & $\Delta E(\mathrm{GeV})$ & $\phi_{\nu_{\mu}}$ & $\delta \phi_{\nu_{\mu}}^{H P}$ \\
\hline$[0,0.1]$ & 0.25 & 31.3 & {$[0.1,0.2]$} & 0.62 & 23.0 \\
\hline$[0.2,0.3]$ & 0.82 & 16.7 & {$[0.3,0.4]$} & 1.00 & 13.3 \\
\hline$[0.4,0.5]$ & 1.19 & 11.7 & {$[0.5,0.6]$} & 1.38 & 10.9 \\
\hline$[0.6,0.7]$ & 1.56 & 10.4 & {$[0.7,0.8]$} & 1.70 & 10.0 \\
\hline$[0.8,0.9]$ & 1.84 & 9.7 & {$[0.9,1]$} & 2.03 & 9.3 \\
\hline$[1,1.1]$ & 2.30 & 9.1 & {$[1.1,1.2]$} & 2.71 & 8.8 \\
\hline$[1.2,1.3]$ & 3.36 & 8.5 & {$[1.3,1.4]$} & 4.26 & 8.3 \\
\hline$[1.4,1.5]$ & 5.14 & 8.1 & {$[1.5,1.6]$} & 5.93 & 8.0 \\
\hline$[1.6,1.7]$ & 6.63 & 7.9 & {$[1.7,1.8]$} & 7.18 & 7.9 \\
\hline$[1.8,1.9]$ & 7.47 & 7.8 & {$[1.9,2]$} & 7.43 & 7.7 \\
\hline$[2,2.1]$ & 7.02 & 7.7 & {$[2.1,2.2]$} & 6.29 & 7.7 \\
\hline$[2.2,2.3]$ & 5.38 & 7.6 & {$[2.3,2.4]$} & 4.44 & 7.5 \\
\hline$[2.4,2.5]$ & 3.59 & 7.5 & {$[2.5,2.6]$} & 2.87 & 7.4 \\
\hline$[2.6,2.7]$ & 2.31 & 7.3 & {$[2.7,2.8]$} & 1.85 & 7.2 \\
\hline$[2.8,2.9]$ & 1.47 & 7.3 & {$[2.9,3]$} & 1.17 & 7.2 \\
\hline$[3,3.1]$ & 0.93 & 7.4 & {$[3.1,3.2]$} & 0.73 & 7.5 \\
\hline$[3.2,3.3]$ & 0.58 & 8.1 & {$[3.3,3.4]$} & 0.46 & 8.4 \\
\hline$[3.4,3.5]$ & 0.37 & 8.7 & {$[3.5,3.6]$} & 0.32 & 9.2 \\
\hline$[3.6,3.7]$ & 0.28 & 9.8 & {$[3.7,3.8]$} & 0.25 & 10.0 \\
\hline$[3.8,3.9]$ & 0.23 & 10.0 & {$[3.9,4]$} & 0.21 & 10.0 \\
\hline$[4,4.1]$ & 0.20 & 10.6 & {$[4.1,4.2]$} & 0.18 & 10.6 \\
\hline$[4.2,4.3]$ & 0.18 & 10.7 & {$[4.3,4.4]$} & 0.16 & 10.7 \\
\hline$[4.4,4.5]$ & 0.15 & 10.5 & {$[4.5,4.6]$} & 0.15 & 10.7 \\
\hline$[4.6,4.7]$ & 0.14 & 10.2 & {$[4.7,4.8]$} & 0.14 & 10.0 \\
\hline$[4.8,4.9]$ & 0.13 & 10.3 & {$[4.9,5]$} & 0.13 & 10.8 \\
\hline$[5,5.1]$ & 0.12 & 10.3 & {$[5.1,5.2]$} & 0.11 & 10.7 \\
\hline$[5.2,5.3]$ & 0.11 & 10.0 & {$[5.3,5.4]$} & 0.11 & 10.5 \\
\hline$[5.4,5.5]$ & 0.10 & 10.4 & {$[5.5,5.6]$} & 0.10 & 10.4 \\
\hline$[5.6,5.7]$ & 0.09 & 9.8 & {$[5.7,5.8]$} & 0.09 & 10.2 \\
\hline$[5.8,5.9]$ & 0.09 & 10.1 & {$[5.9,6]$} & 0.09 & 9.8 \\
\hline \hline
\end{tabular}

TABLE B.4: Generation2 - thin Medium Energy flux results at the center of the front face of NOvA Near Detector. Flux units: $\nu / m^{2} / 10^{6} P O T$. Uncertainties are given in percentage. 


\section{BIBLIOGRAPHY}

[1] Paul Langacker, The Standard Model and Beyond (CRC Press, 2009).

[2] M. Fukugita and T. Yanagida, Physics of Neutrinos and Applications to Astrophysics (Germany, 2003).

[3] K. O. et al. (Particle Data Group), Chin. Phys. C 38, 090001 (2014).

[4] F. C. et al., Phys. Rev. D 89, 093018 (2014).

[5] P. Adamson et al. (MINOS Collaboration), Phys. Rev. D 77, 072002 (2008).

[6] J. A. Formaggio and G. P. Zeller, Rev. Mod. Phys. 84, 1307 (2012).

[7] M. T. et al., Phys. Rev. D 74, 012008 (2006).

[8] Dune Collaboration, Long-Baseline Neutrino Facility (LBNF) and Deep Underground Neutrino Experiment (DUNE) Conceptual Design Report, FERMILABDESIGN-2016-02.

[9] MINERvA Collaboration, MINERvA-doc-700-v28.

[10] A. Higuera et al. (MINERvA Collaboration), Phys. Rev. Lett. 113, 261802 (2014).

[11] Sacha E. Kopp, Phys. Rept 439, 101 (2007).

[12] Van der Meer, S. and Vahlbruch, K. M., CERN-1963-037 pp. 97-110 (1963). 
[13] P. Adamson et. al., Nucl. Instrum. Meth. A806, 279 (2016).

[14] NuMI/ MINOS, NuMI technical design handbook, Accessed: 2016-01-25, URL http://www-numi.fnal.gov/numwork/tdh/tdh_index.html.

[15] Fermilab, Accelerator report no. 4: The linac, Accessed: 2016-01-25, URL http://www.fnal.gov/pub/news04/update_archive/update_10-1.html.

[16] Fermilab, Booster department, Accessed: 2016-02-02, URL http://www-ad. fnal.gov/proton/booster.html.

[17] C. C. Jensen, G. A. Krafczyk (Fermilab), Conf. Proc. C0505161, 692 (2005).

[18] K. Budal, IEEE Trans. Nucl. Sci. 14, 1132 (1967).

[19] Fermilab, Visual media service, Accessed: 2016-02-22, URL http://vms .fnal. gov.

[20] GEANT4 Collaboration, Nucl. Instrum. Meth. A506, 250 (2003).

[21] GEANT4, Model: Ftfp, Accessed: 2016-02-11, URL http://geant4.cern.ch/ support/proc_mod_catalog/models/hadronic/FTFP.html.

[22] GEANT4, Model: Bertini cascade, Accessed: 2016-02-11, URL http://geant4.cern.ch/support/proc_mod_catalog/models/hadronic/ BertiniCascade.html.

[23] C. Andreopoulos et al, Nucl. Instrum. Meth. A614, 87 (2010).

[24] MINOS Collaboration, The MINOS technical design report, Accessed: 2016-0301, URL http://www-numi.fnal.gov/minwork/info/minos_tdr.html.

[25] Ž. Pavlović, Ph.D. thesis (2008), FERMILAB-THESIS-2008-59.

[26] M. Kordosky, MINERvA-doc-9709-v3. 
[27] P. Le Brun, Tech. Rep. (2014), MINERvA-doc-10573-v1.

[28] J. Hylen, MINERvA-doc-9646-v1.

[29] P. Le Brun, Tech. Rep. (2014), MINERvA-doc-10406-v3.

[30] L. J. Loiacono, Ph.D. thesis (2011), FERMILAB-THESIS-2011-06.

[31] M. Apollonio et al. (HARP Collaboration), Phys. Rev. C 80, 035208 (2009).

[32] M. Apollonio et al. (HARP Collaboration), Nuclear Physics A 821, 112 (2009).

[33] M. Apollonio et al. (HARP Collaboration), Phys. Rev. C 82, 045208 (2010).

[34] M. Apollonio et al. (HARP Collaboration), Eur. Phys. J. C 52, 29 (2007).

[35] D. W. Schmitz, Ph.D. thesis (2008), FERMILAB-THESIS-2008-26.

[36] D. A. A. Wickremasinghe, Ph.D. thesis (2015), FERMILAB-THESIS-2015-23.

[37] Fermilab, Booster neutrino experiment, Accessed: 2016-03-8, URL https:// www-boone. fnal.gov/.

[38] A. A. Aguilar-Arevalo et al. (MiniBooNE Collaboration), Phys. Rev. D 79, $072002(2009)$.

[39] K. Abe et al. (T2K Collaboration), Phys. Rev. D 87, 012001 (2013).

[40] N. Abgrall et al. (NA61/SHINE Collaboration), Phys. Rev. C 84, 034604 (2011).

[41] N. Abgrall et al. (NA61/SHINE Collaboration), Phys. Rev. C 85, 035210 (2012).

[42] N. Abgrall et al. (NA61/SHINE Collaboration), Nucl. Instrum. Meth. A701, 99 (2013). 
[43] Bonesini, M. et al., Eur. Phys. J. C20, 13 (2001).

[44] P. Adamson et al. (MINOS Collaboration), Phys. Rev. D 81, 072002 (2010).

[45] A. Bodek, U. Sarica, D. Naples, L. Ren, The European Physical Journal C 72-4, 1973 (2012).

[46] W. G. Seligman, Ph.D. thesis (1997), FERMILAB-THESIS-1997-21.

[47] D. Bhattacharya, Ph.D. thesis (2009), FERMILAB-THESIS-2009-11.

[48] J. Devan, Ph.D. thesis (2015), FERMILAB-THESIS-2015-29.

[49] J. Park, Ph.D. thesis (2013), FERMILAB-THESIS-2013-36.

[50] R. P. Feynman, Phys. Rev. Lett. 23, 1415 (1969).

[51] J. Benecke, T.T. Chou, C.N. Yang and E. Yen, Phys. Rev. 188, 2159 (1969).

[52] T.T. Böhlen, F. Cerutti, M.P.W. Chin, A. Fassò, A. Ferrari, P.G. Ortega, A. Mairani, P.R. Sala, G. Smirnov and V. Vlachoudis, Nuclear Data Sheets 120, $211(2014)$.

[53] A. Ferrari, P.R. Sala, A. Fasso; and J. Ranft, CERN-2005-10, INFN/TC_05/11, SLAC-R-773 (2005).

[54] M. Kordosky, MINERvA-doc-7397-v1.

[55] J. Wolcott, Tech. Rep. (2013), MINERvA-doc-8628-v2.

[56] D. S. Barton et al., Phys. Rev. D 27, 2580 (1983).

[57] P. Skubic, et al., Phys. Rev. D 18, 3115 (1978).

[58] T. Golan, MINERvA-doc-11150-v1. 
[59] M. Jerkins, Tech. Rep. (2012), MINERvA-doc-7633-v1.

[60] M. Kordosky, Tech. Rep. (2012), MINERvA-doc-7433-v1.

[61] W.H.Press et Al., Numerical Recipes. The Art of Scientific Computing (Cambridge, University Press, 2007), 3rd ed.

[62] J. Paley et Al. (MIPP Collaboration), Phys. Rev. D 90, 032001 (2014).

[63] S. M. Seun, Ph.D. thesis (2007), FERMILAB-THESIS-2007-61.

[64] G. Bellettini et al., Nucl. Phys. 79, 609 (1966).

[65] S. P. Denisov et al., Nucl. Phys. B61, 62 (1973).

[66] NA49 Collaboration, Eur. Phys. J. C49, 897 (2007).

[67] NA49 Collaboration, Eur. Phys. J. C73, 2364 (2013).

[68] G. M. Tinti, Ph.D. thesis (2010), FERMILAB-THESIS-2010-44.

[69] A. V. Lebedev, Ph.D. thesis (2007), FERMILAB-THESIS-2007-76.

[70] NA49 Collaboration, Nucl. Instrum. Meth. A430, 210 (1999).

[71] CERN, Large acceptance hadron detector for an investigation of pb-induced reactions at the cern sps, Accessed: 2016-03-06, URL http://na49info.web. cern.ch.

[72] A. Zichichi, Understanding the Fundamental Constituents of Matter (Plenum Press, 1978).

[73] A. S. Carroll et al., Physics Letter B, 319 (1979).

[74] T. J. Roberts et al., Nuclear Physics B159, 56 (1979). 
[75] J. W. Cronin et al., Phys. Rev. 107, 1121 (1957).

[76] J. V. Allaby et al., Yad. Fiz. 12, 538 (1970).

[77] B. W. Allardyce et al., Nuclear Physics 209, 1 (1973).

[78] M. Longo et al., Phys. Rev. 125, 701 (1962).

[79] V. B. Fedorov et al., Sov. J. Nucl. Phys. 222, 413 (1978).

[80] B. M. Bobchenko et al., Sov. J. Nucl. Phys. 30, 805 (1979).

[81] R. J. Abrams et al., Phys. Rev. D1, 1917 (1970).

[82] R. Hatcher, MINOS-doc-9070-v4.

[83] L. Aliaga, MINERvA-doc-10814-v1.

[84] L. Aliaga, MINERvA-doc-11154-v1.

[85] L. Aliaga, MINERvA-doc-11415-v2.

[86] G. A. Fiorentini et al. (MINERvA Collaboration), Phys. Rev. Lett. 111, 022502 (2013).

[87] L. Fields et al. (MINERvA Collaboration), Phys. Rev. Lett. 111, 022501 (2013).

[88] B.G. Tice et al. (MINERvA Collaboration), Phys. Rev. Lett. 112, 231801 (2014).

[89] T. Walton et al. (MINERvA Collaboration), Phys. Rev. D. 91, 071301 (2015).

[90] T. Le et al. (MINERvA Collaboration), Phys. Lett. B. 749, 130 (2015).

[91] B. Eberly et al. (MINERvA Collaboration), Phys. Lett. D. 92, 092008 (2015).

[92] M. Kordosky, MINERvA-doc-11713-v4. 
[93] J. Park, MINERvA-doc-10972-v2.

[94] L. Fields, MINERvA-doc-11668-v6.

[95] J. Wolcott et al. (MINERvA Collaboration), Phys. Rev. D 81, 072002 (2010).

[96] A. Radovic for the NuMIX working group, NUMIX-doc-31-v4. 


\title{
VITA
}

\author{
Leonidas Aliaga
}

Leonidas Aliaga-Soplin is a native of Lima, Peru. He graduated from Pontificia Universidad Catolica Del Peru, in Lima, Peru in 2008 in M.S Physics. He began his graduate studies at William and Mary in the fall of 2009, where he started working as a collaborator in MINERvA experiment under the supervision of Dr. Mike Kordosky. His research has been focused on the NuMI flux determination primarily for MINERvA but also extending to other Fermilab neutrino detectors. After graduating, Leonidas will begin working at the Fermilab Scientific Computing Division at Batavia, IL on the NOvA experiment. 